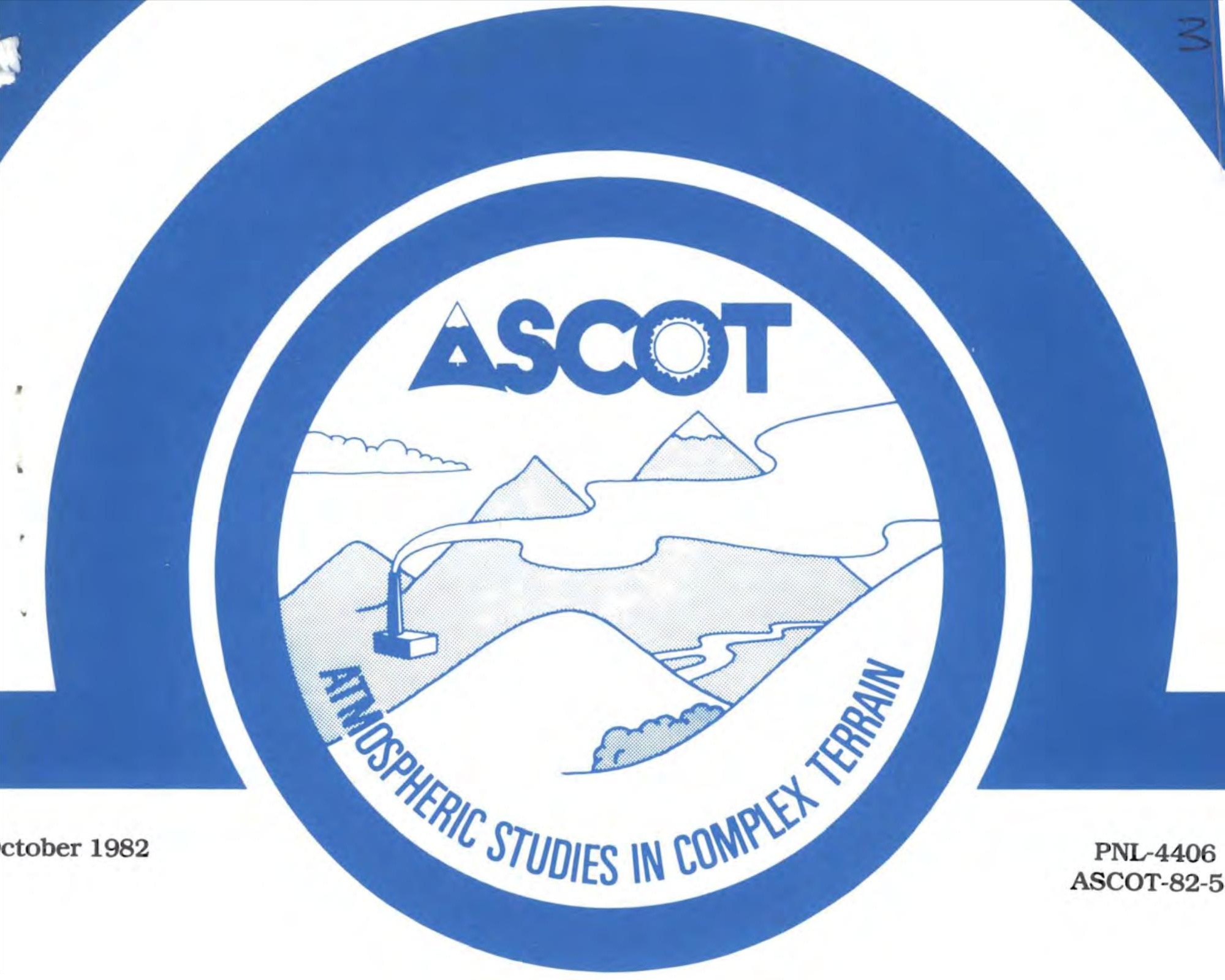

\title{
Simple Nocturnal Slope Flow Data From the Rattlesnake Mountain Site
}

\author{
T.W. Horst \\ J.C. Doran
}

Pacific Northwest Laboratory

Richland, Washington 


\title{
DISCLAIMER
}

This report was prepared as an account of work sponsored by an agency of the United States Government. Neither the United States Government nor any agency thereof, nor any of their employees, makes any warranty, express or implied, or assumes any legal liability or responsibility for the accuracy, completeness, or usefulness of any information, apparatus, product, or process disclosed, or represents that its use would not infringe privately owned rights. Reference herein to any specific commercial product, process, or service by trade name, trademark, manufacturer, or otherwise, does not necessarily constitute or imply its endorsement, recommendation, or favoring by the United States Government or any agency thereof. The views and opinions of authors expressed herein do not necessarily state or reflect those of the United States Government or any agency thereof.

\author{
PACIFIC NORTHWEST LABORATORY \\ operated by \\ BATTELLE \\ for the \\ UNITED STATES DEPARTMENT OF ENERGY \\ under Contract DE-AC06-76RLO 1830
}

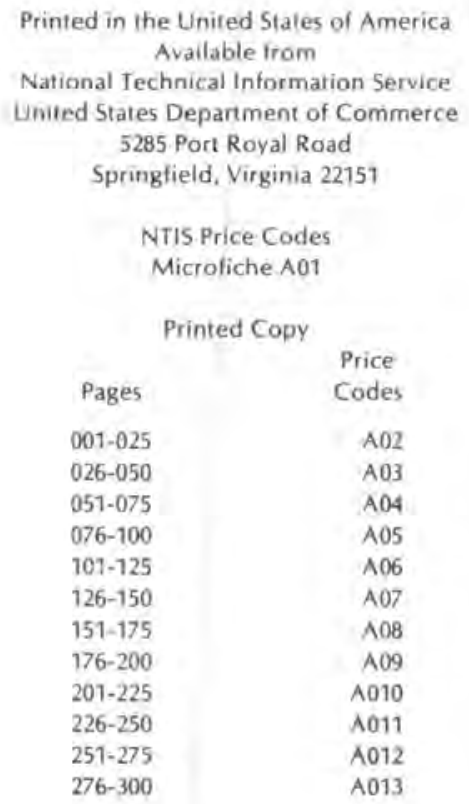


T.W. HORST

J.C. DORAN

OCTOBER 1982

Prepared for

the U.S. Department of Energy

under Contract DE-ACO6-76RLO 1830

Pacific Northwest Laboratory Richland, Washingt on 99352 


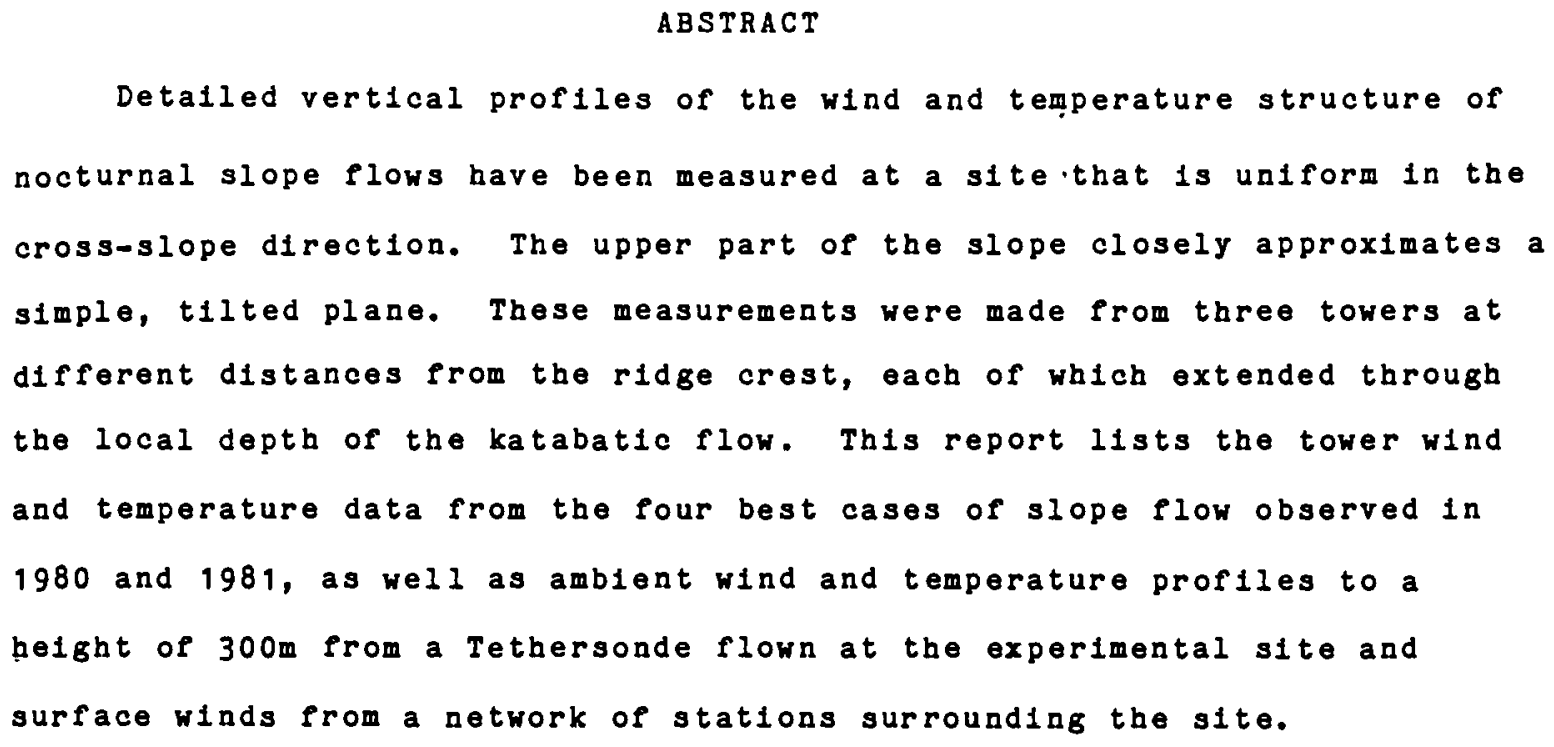




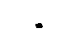

.

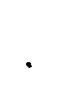

. 
I. Introduction

II. Site and Instrumentation

III. Case Description

October 16,1981

IV. Tower Data

June 19-20, 1980

July 1-2, 1980

July 2-3, 1981

October 16, 1981

V. Tethersonde Data

June 19-20, 1980

July $1-2,1980$

July $2-3,1981$

October 16,1981

VI. Telemetry Network Data

June 19-20, 1980

July $1-2,1980$

July $2-3,1981$

October 16,1981 
1. Topography and cross-section of the Rattiesnake site

2. Hanford telemetry station sites

3. Hanford streamline analysis, 2100 June 19, 1980

4. Hanford streamline analysis, $0000 \mathrm{July} \mathrm{2,1980}$

5. Hanford streamline analysis, $2300 \mathrm{July} \mathrm{2,} 1981$

6. Hanford streamline analys1s, 2000 october 16, 1981

\section{LIST OF TABLES}

TABLE

PAGE

1. Locations of Rattiesnake observation sites

2. Nominal heights of wind and temperature measurements

3. Locations of Hanford telemetry stations

4. 1980 Instrument heights for tower $C$ 
ACRN OWLEDG EMENTS

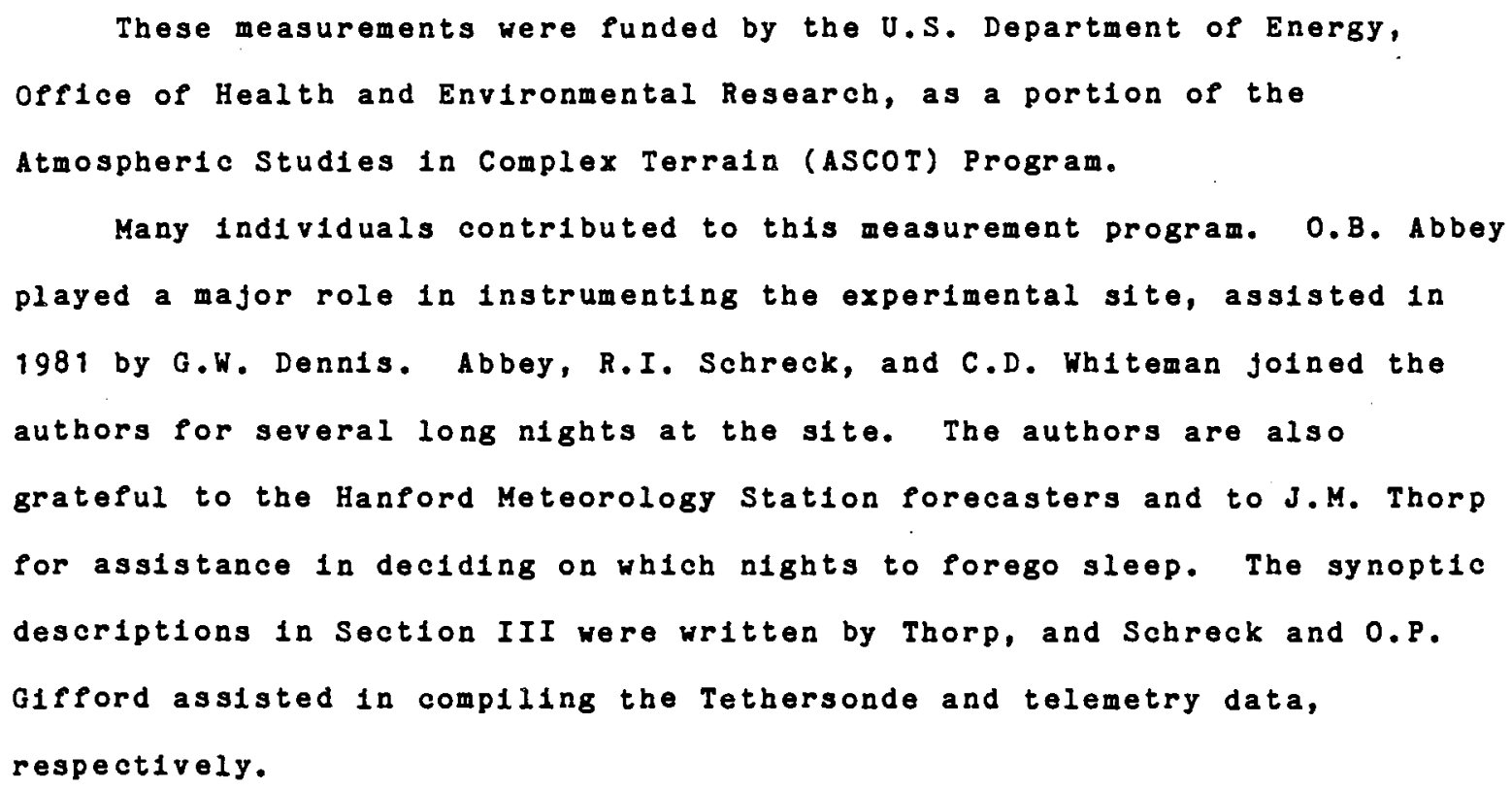




\section{INTRODUCTION}

Measurements of the wind and temperature structure of nocturnal slope flow were made on Rattlesnake Mountaln near Richland, Washington during the summers of 1980 and 1981. These measurements were made as part of Project ASCOT (Atmospheric Studies in Complex Terrain), a multi-laboratory research effort sponsored by the U. S. Department of Energy.

AScot's initial focus has been on nocturnal drainage winds. Drainage winds begin when alr adjacent to a sloped surface plows down the slope because it is cooled more, and is denser than, the free alr at some distance from the surface. These slope winds commonly merge and are channeled by the topography to form larger-scale drainage or mountain winds, but the Rattlesnake Mountaln site was selected for measurements of slope flow in the simplest possible geometry.

Th1s report l1sts the data from the four best cases observed during 1980 and 1981: June 19-20 and July 1-2, 1980, and July 2-3 and october 16, 1981. The measurement site and instrumentation are described in Section II and the four cases are described in section III. The actual data I1stings are contained in Sections IV-VI.

\section{SITE AND INSTRUMENTATION}

The Rattlesnake Mountain site used for these measurements forms a nearly 1deal tilted plane, covered with 20-30 cm bunchgrass and scattered sagebrush with helghts of 1-1.5 m. The estimated roughness length of the site is of the order of a few cm. A ridge 595m abovem.s.l. runs for nearly $2 \mathrm{~km}$ from northwest to southeast. To the northwest the ridge then rises to a height of $1087 \mathrm{~m} \mathrm{m.s.1.} \mathrm{In} 6.3 \mathrm{~km}$, and falls to $140 \mathrm{~m} \mathrm{m.s.1.} \mathrm{at} \mathrm{a}$ distance of $2.7 \mathrm{~km}$ to the southeast. Within the level $2 \mathrm{~km} \mathrm{stretch,} \mathrm{the}$ slope falls away quite uniformly to the northeast for a vertical drop of $170 \mathrm{~m}$, with an average slope of 21 degrees. At this point there $1 \mathrm{~s}$ a rather abrupt change in the slope to an angle of approximately 8 degrees, after which there is a continued, gradual leveling of the pitch (Figure 1). 
The detalled topography of the site may be found on U.S. Geological Survey maps. The site 1s located near the NE corner of the $71 / 2$ corral Canyon quadrangle at UTM coordinates $5136 \mathrm{~N}$ and 306 E. Surrounding $71 / 2$ ' maps are (clockwise from north): Iowa Flats, Horn Rapids, Benton C1ty, Webber Canyon, Whitstran NE, Whitstran, Snipes Creek, and Snively Basin. The $71 / 2$ ' Corral Canyon map is the SE quarter of the 15 ' Corral Canyon quadrangle. Surrounding 15' maps are: Coyote Rapids, Hanford, Richland, Badger Mountaln, Whitstran, Prosser, Grandview, and Priest Rapids.

Three towers, labeled A, B, and C In Figure 1, were erected on the Rattlesnake site to measure the development of slope flow with downslope distance. Towers $A$ and $B$ were placed on the upper, steeper slope, and a Tethersonde base station and tower $C$ were located on the lower slope. For each observation site, Table 1 lists the elevation abovem.s.1., the az1muth from bench mark 1109 (at the Intersection of sections $11,12,13,14$ of T1ON, R26E), the vertical drop from the top of the ridge, the local slope angle, and the distance along the slope from the ridge top to the site.

Table 1. Locations of Rattiesnake observation sites. Stie Elevation Azimuth Lertical Deop Slope Ansle slepe Distance

$\begin{array}{cccccc}\text { A } & 526 \text { m } & 228 \mathrm{deg} & 69 \mathrm{~m} & 21 \mathrm{deg} & 193 \mathrm{~m} \\ \text { B } & 444 & 226 & 151 & 21 & 422 \\ \text { T-sonde } & 401 & 222 & 194 & 8 & 647 \\ \text { C } & 366 & 211 & 229 & 8 & 898\end{array}$

Towers $B$ and $C$ were instrumented w1th Gill 3 component anemometers and aspirated YSI thermistors. The G1Il anemometers used 23 cm diameter propeliors with a threshold of 10-20 cm/sec and a response distance of 1m. The aspirated YSI thermistors bave a response time of $2-3$ sec. Durling the spring and summer of 1980, tower A was equipped only with thermistors, but in 1981 these were replaced with climet cups, vanes and aspirated thermistors. The CIImet cups (model 011-1) have a threshold of 


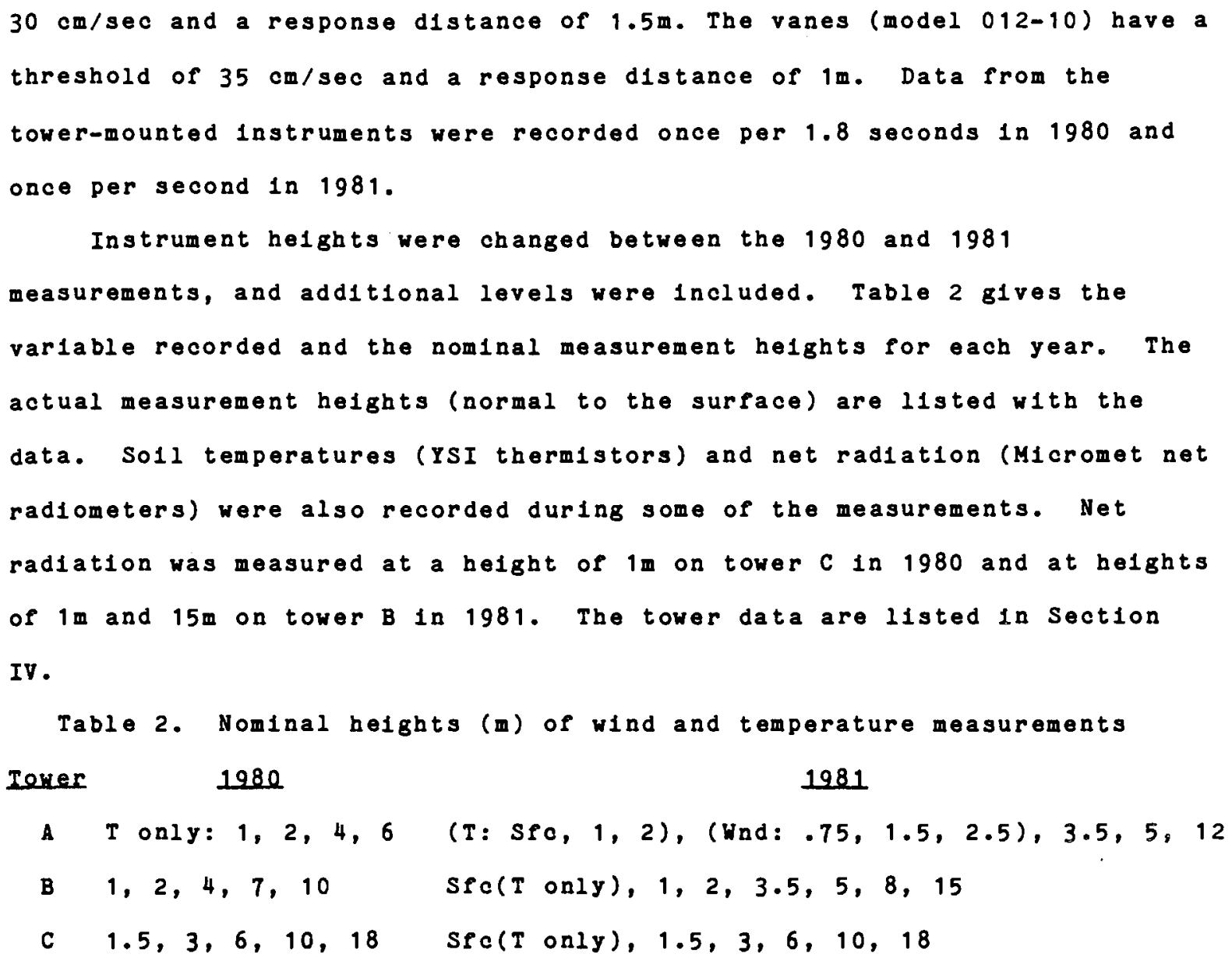

The Tethersonde was used to monitor ambient wind speed and direction, temperature and relative humldity up to helghts $500 \mathrm{~m}$ above the base station. Data were recorded once every 10-20 seconds from the Tethersonde. A complete profile (up and down) with the sonde was taken approximately once per hour. The Tethersonde data are listed in section v. Supplementry surface winds are available from the Hanford Telemetry Network shown in Figure 2. This network consists of 22 stations deployed around the Hanford reservation and roughly centered on Station 21 , the Hanford Meteorology Station (HMS). Each station transmits hourly averages of wind speed and direction measured at a helght of $9.15 \mathrm{~m}$. Table 3 lists the station names, locations and elevations. The Rattlesnake experimental site is located in the south-central portion of the network, about $24 \mathrm{~km}$ SSE Of HMS. 

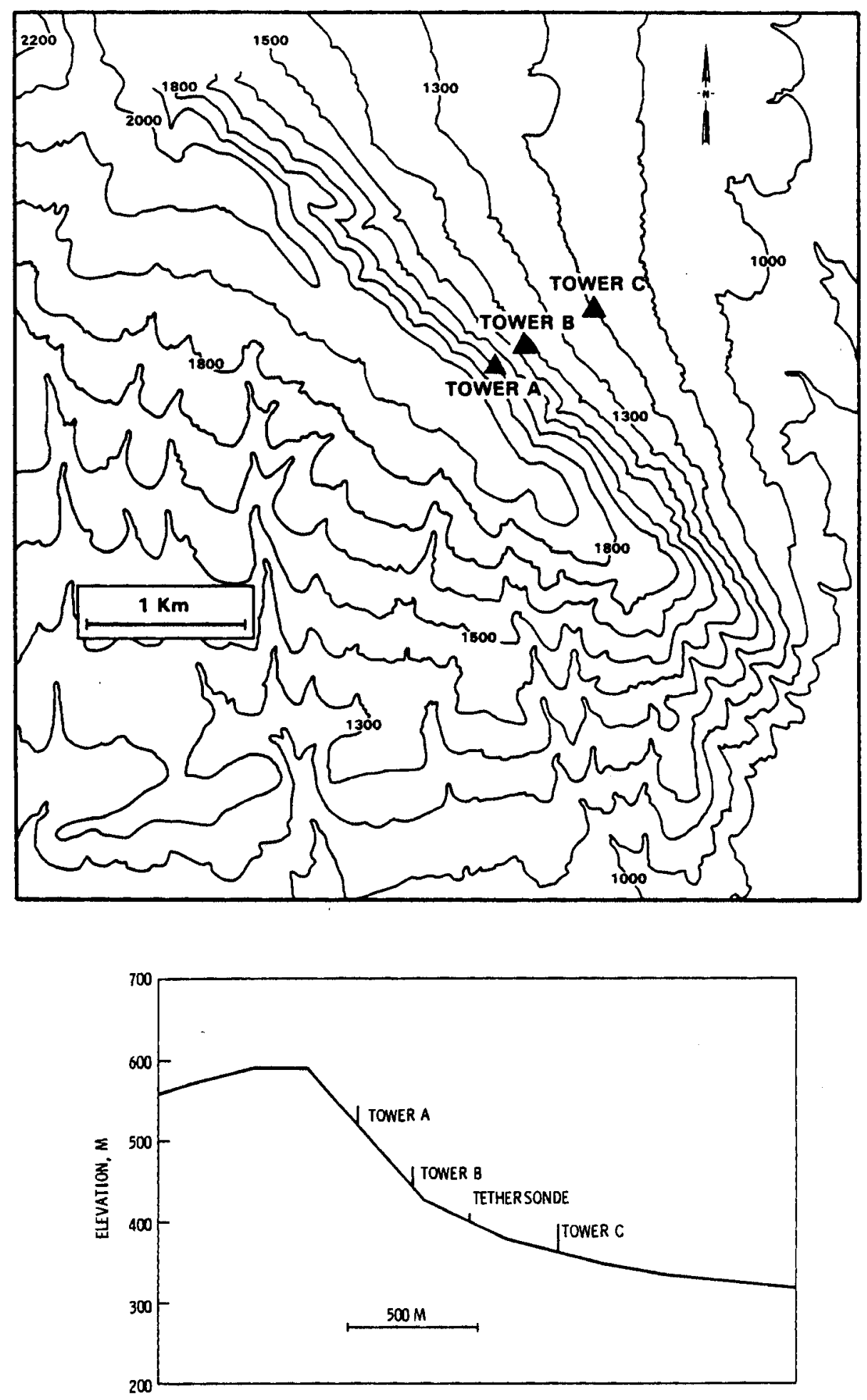

Figure 1. Topography and cross-section of the Rattlesnake site 


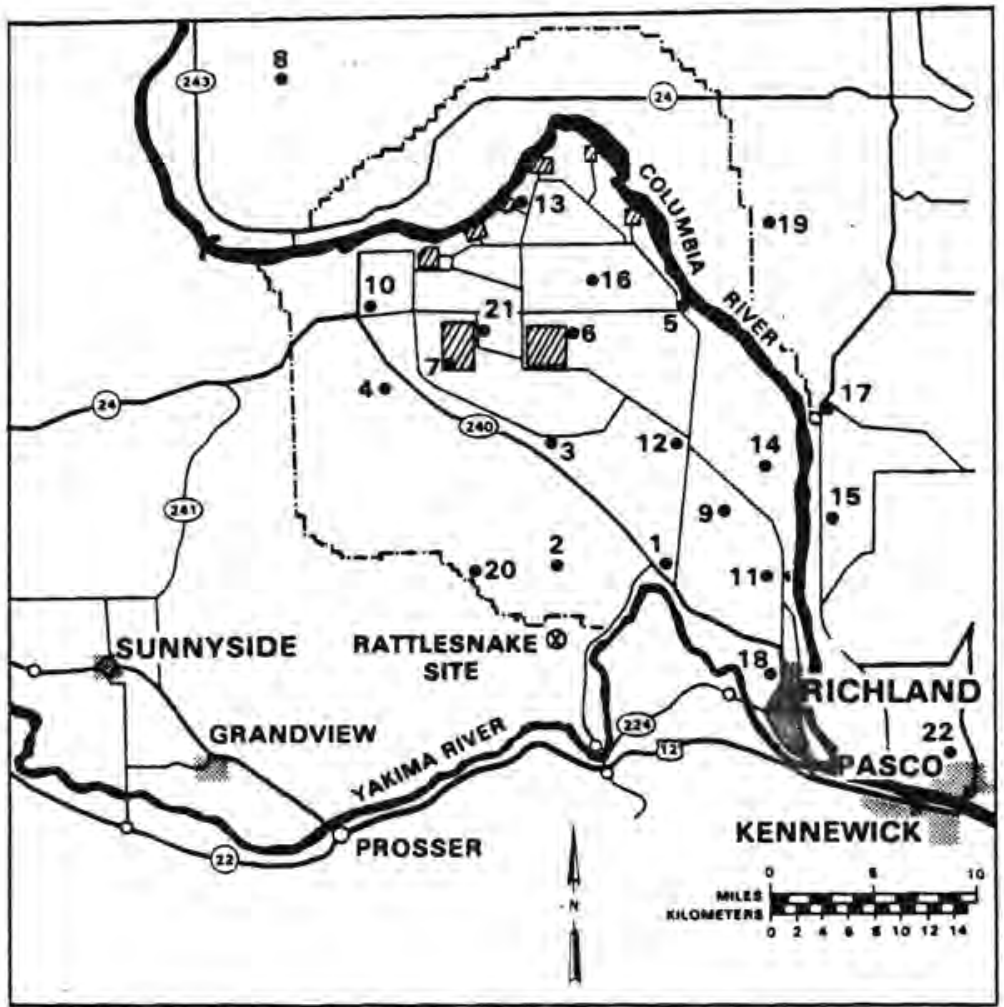

Figure 2. Hanford telemetry station sites

Table 3. Locations of Hanford telemetry stations

\section{Station}

2

3

4

5

6

7

\section{Name}

Prosser Barricade
EOC
Army Loop Road
Rattlesnake Springs
Edna
$200 E$
$200 W$
Wahluke
FFTF
Yak1ma Barricade
300 Area
WYE Barricade
100N
WPPSS
Frankl1n County
Gable Mt.
Ringold
Richland Alrport
Sagehill
Rattlesnake Mt.
HMS
Pasco A1rport

\begin{tabular}{r} 
Elevation \\
MSL(m) \\
\hline 146 \\
378 \\
172 \\
207 \\
122 \\
204 \\
201 \\
258 \\
168 \\
242 \\
122 \\
168 \\
146 \\
137 \\
269 \\
331 \\
189 \\
119 \\
300 \\
1087 \\
223 \\
122
\end{tabular}

OTM Coordinates Zone 11 $\mathrm{km}$ North km East

$\begin{array}{ll}5140.20 & 314.65 \\ 5140.60 & 305.00 \\ 5151.30 & 304.45 \\ 5153.60 & 292.90 \\ 5161.80 & 316.40 \\ 5158.70 & 306.80 \\ 5157.40 & 298.35 \\ 5179.35 & 283.30 \\ 5145.15 & 319.20 \\ 5161.65 & 291.20 \\ 5136.80 & 324.20 \\ 5150.20 & 316.40 \\ 5171.90 & 304.00 \\ 5148.70 & 320.15 \\ 5142.60 & 328.05 \\ 5163.50 & 311.20 \\ 5156.75 & 328.50 \\ 5129.85 & 322.85 \\ 5169.25 & 324.10 \\ 5140.90 & 300.60 \\ 5159.70 & 300.85 \\ 5125.20 & 336.90\end{array}$


The telemetry network data are listed in section VI. Streamilne analysis of this data is shown for a selected hour during each experiment In Figures 3-6. It should be noted that the wind speed at station 20 is treated separately in these analyses. This station, situated on the narrow ridge crest of Rattlesnake Mt. at $1087 \mathrm{~m}$, is more representative of the synoptic than the local scale flows.

II. Case Descriotion

The data selected for inclusion in this document were taken during four aights of observation. In each case there is evidence of the occurrence of dralnage winds for at least a portion of the observation period. The criterion used for this identification is the presence of a Jet, 1.e., a local maximum in the vertical profile of the downslope component of the tower wind. The downslope component u corresponds to a direction of 225 degrees (wind from SW) and the cross-slope component v corresponds to 135 degrees (wind from SE). In general there 1 a significant cross-slope wind at all times, although its magnitude relative to the u component varies considerably. Pure slope winds, devold or any cross slope "contamination", were not obtained.

For each case a brief summary is given below of the synoptic situation, the surface winds over the Hanford area from the telemetry network, the Tethersonde observations at the experimental site, and the wind and temperature proflles from tower B. Tower B was located on the portion of the slope that was relatively constant in angle right up to the ridge line, whereas tower c was below the point at which the slope had a discontinuity in angle. Hence, the data at tower B are more properly representative of simple slope characteristics, while that at tower C would be expected to exhibit convergence effects.

All times are Pacific Standard Time (PST). 
Iune 19-20 (Days 171-172). 1980: 184i-0251 RST

At 1600 PST, June 19 a rather weak high pressure area was of the WA coast. Inland a thermal trough extended from CA up through central or, and there was a small low in southern BC as an extension of the trough. The sea-level pressure difference between Seattle and Hanford was only -3mb. Surface winds at reporting stations in eastern WA were easterly to southerly and light. Broken to overcast high cloudiness covered the Hanford area, but was thin except for 3 hours after 1900 PST.

By 2200 on June 19 the thermal trough had moved about 100 miles east and was now in eastern $O R$ with the northern portion over the Hanford area. Pressure differences remained at $-3 m b$ between Seattle and Hanford and -2mb between Spokane and Hanford. The temperature which had been 30 deg C at 1600 had cooled to 21 deg C at 2200. Shortly after midnight altocumulus and altocumulus castellatus clouds were reported at the Hanford Meteorology Station (HMS), indicating an increase of molsture at intermediate levels.

By 0400 on June 20 the thermal trough had broadened into western ID. Pressure differences between Seatte and Hanford and between Spokane and Hanford were $-3.8 m b$ and $-2.2 m b$, respectively -- still quite weak. The layer of intermediate cloudiness observed at HMS persisted and had become broken by 0500 PST.

Surface winds at HMS were light and varlable until midnight; after mldnlght they were $2-4 \mathrm{~m} / \mathrm{s}$ from the $W$ and $N$. Before sunset low-level wind flow at telemetry stations near the experimental site was from the $\mathrm{NE}$ to $\mathrm{E}$ with speeds of $2.5 \mathrm{~m} / \mathrm{s}$ or less. After sunset the wind shifted to between $W$ and $N$ with speeds of 2-3 mss. Thls flow continued for the remalnder of the night. During the perlod 2000-2200 PST a slight backlng of the low-level wind from northerly to more northwesterly was observed.

At the experimental site, Tethersonde ascents were made at roughly 2300, 0000,0045 and 0130 PST. They showed a surface wind direction of WN, shifting to $N$ in the first $50 \mathrm{~m}$ above the surface and then to $N E$ at a 


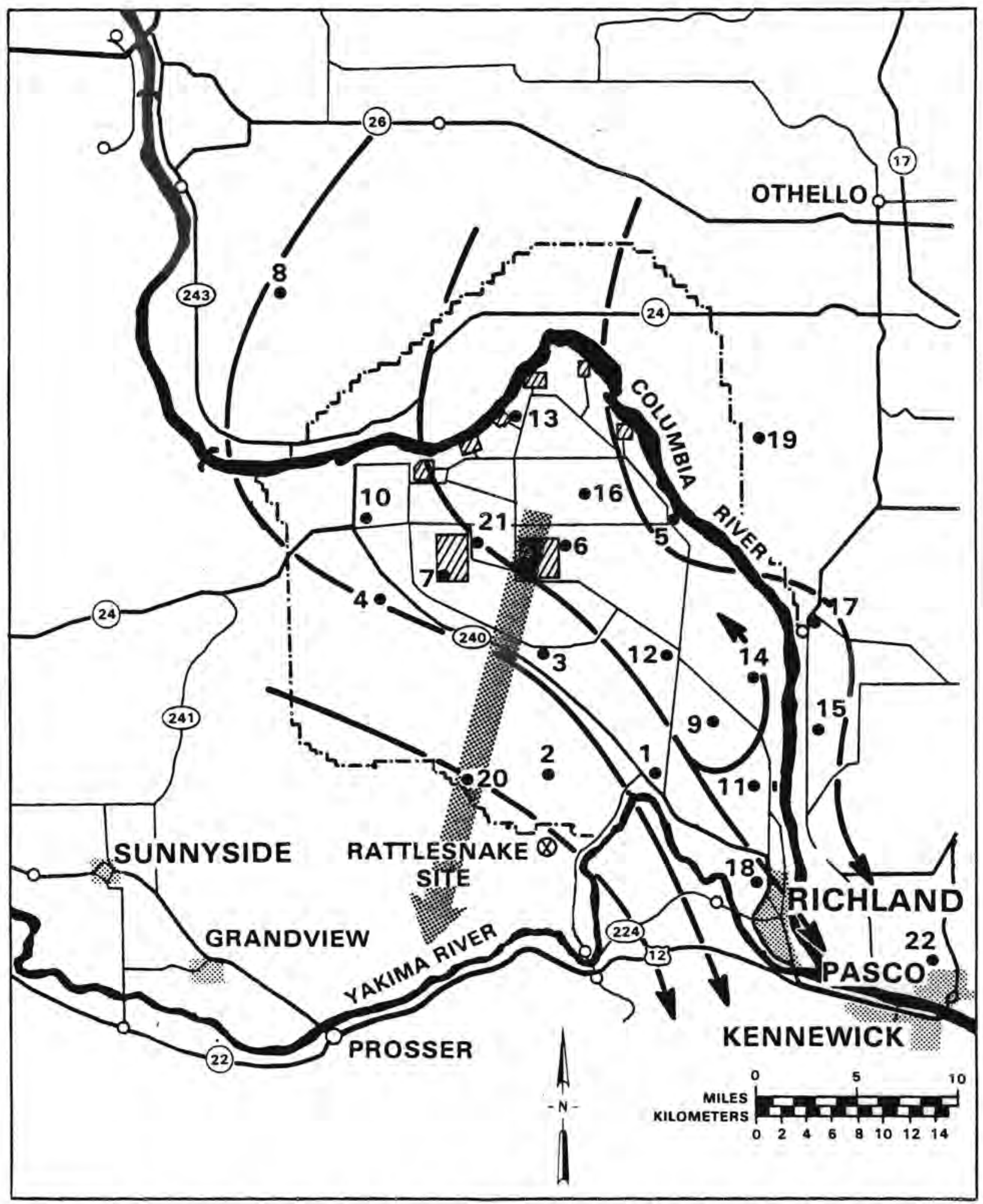

F1gure 3. Hanford streamline analys1s, 2100 June 19, 1980 


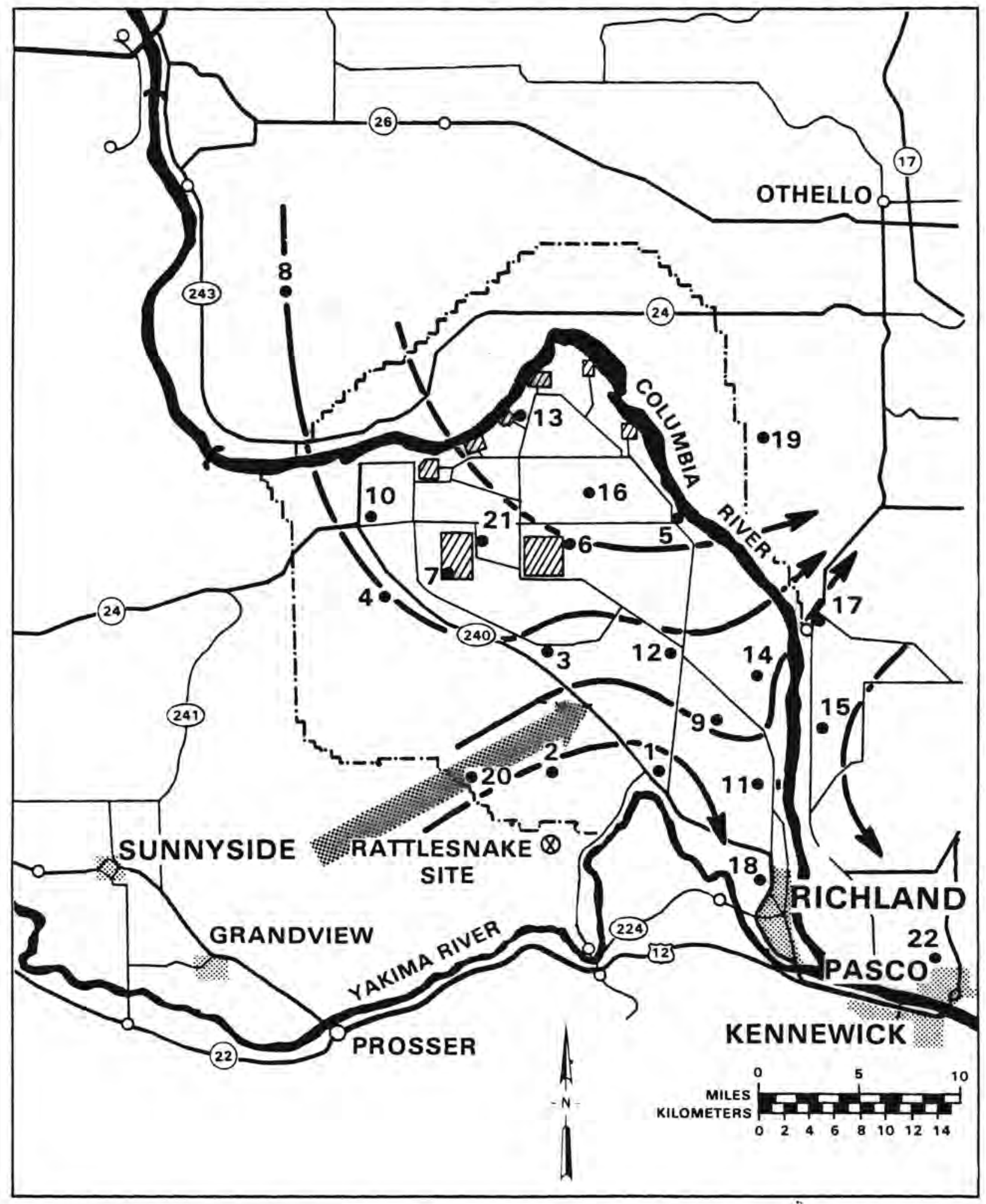

Figure 4. Hanford streamline analysis, $0000 \mathrm{July} \mathrm{2,1980}$ 
height of 250-350m. The wind speed between $25 \mathrm{~m}$ and $300 \mathrm{~m}$ was initially 3-7 m/s but decreased after midnight to $2-4 \mathrm{~m} / \mathrm{s}$. The potential temperature profile from $50 \mathrm{~m}$ to $300 \mathrm{~m}$ was adiabatic to stable at $1-2 \mathrm{~K} / \mathrm{km}$.

At tower $B$ there was steady cooling at $0.5 \mathrm{deg} \mathrm{C} / \mathrm{hr}$ from 1830 until 0100, after which the temperature remained falrly constant. The inversion strength, $T(9 \mathrm{~m})-T(1.2 \mathrm{~m})$, increased from 0.5 deg $C$ at 1840 to almost 2 deg C at 1930 , and then decreased slowly to 1.2 deg C after 2130.

From the beginning of the data collection period through 2000 a jet was observed at the $2 m$ level. The cross-slope component was significant, but at the lowest level of measurement it was smaller than $u$. At 2000 the maximum in u dropped to the lowest level, $1.2 \mathrm{~m}$, and stayed there for about 40 minutes, after which it rose to $2 \mathrm{~m}$ again. Around 2040 the $v$ component also began to increase significantly, and by 2110 it was larger than the u component at all levels. By 2230 , the u component at the highest level on tower B was upslope, but a jet remained at 2m. From 2330 until 0200 the jet occured at the lowest level. The $v$ component slowly decreased during this period, but remained strong compared to $u$, and around 0200 began to increase once more.

July 1-2 (Days 183-184), 1980; 1920-0200 PST

At 1600 PST, July 1, a thermal trough of low pressure extended northward east of the Cascade Mountains from OR, through WA and into $B C$. Afternoon temperatures were near $30 \mathrm{deg} C$ in eastern WA and only slightly cooler in the Puget Sound area. Low stratus clouds along the WA coast were beginning to penetrate inland, accompanying cooler air which would reach Puget Sound the following day. The sky was clear in eastern WA all evening on July 1 , allowing 11 deg $C$ of radiational cooling of the HMS $1 \mathrm{~m}$ temperature by midnight. Low level winds were easterly over the Hanford area at 1600 . Northeast winds held steady at $1.5-4 \mathrm{~m} / \mathrm{s}$ until 2200 at HMS, becoming southeasterly at $0-4 \mathrm{~m} / \mathrm{s}$ after that time.

By 2200 PST surface pressure had risen at stations west of the 
Cascades, but In easterm WA little or no change was reported. The Seattle-Hanford pressure difference had Increased from $-2.2 \mathrm{mb}$ at 1600 to $-4.6 \mathrm{mb}$ at 2200 , but the spokane-Hanford pressure gradient Increased by only $0.2 \mathrm{mb}$ during the same perlod. A weak trough was approaching WA from the northwest, bringing an increase in mid level molsture and causing the a1r mass over easterm HA to become more unstable. Alto cumulus and thin c1rrus clouds began to increase after midnight as this trough came closer. At $0200,6 / 10$ thin cloud cover was noted at the experimental site. The middle cloud layer became broken $(>5 / 10)$ by 0300 and effectively terminated the radiational cooling.

The low-level wind at EOC, the telemetry station nearest the experiment site, had slowly backed from the E and was $S$ by 2200 ; this backing trend continued throughout the night and by 0400 PST the wind was WNW at $2 \mathrm{~m} / \mathrm{s}$. The wind direction at the Rattlesnake observatory displayed the same trend, but speeds were in the 7-9 m/s range from 2200 to 0300 . At 0400 on July 2 the Seattle-Hanford pressure difference had Increased to $-6.8 \mathrm{mb}$ and the Spokane-Hanford difference had Increased to $-2.9 \mathrm{mb}$. A closed isobarlc circulation had formed near the Hanford area, and there was evidence of weak frontogenesis taking place in the narrowing trough. Thunderstorms were reported at Redmond, OR and rain showers occured at Hanford in the morning.

Seven Tethersonde ascents were made this night between 1945 and 0130. They showed a surface wind direction of SSW, shifting about 2230 to WSW. This corresponded to a more gradual shift in the wind direction above 100m from SSE to WSW, and a decrease in the ambient wind speed around 2300 from $2-4 \mathrm{~m} / \mathrm{s}$ to $1-2 \mathrm{~m} / \mathrm{s}$. The ambient potential temperature gradient varied from 2 to $4 \mathrm{~K} / \mathrm{km}$.

The $1.2 \mathrm{~m}$ level on tower B cooled at $0.5 \mathrm{deg} \mathrm{C} / \mathrm{hr}$ from 1920 until 0100 , but the $9 \mathrm{~m}$ level did not cool unt1l after 2300 . Consequently the Inversion strength, $T(9 m)-T(1.2 m)$, 1ncreased from 2 to 4 deg $C$ between 1920 and 2130 , stayed constant until 0030 , and then decreased to 3 deg C 
at 0150

From the start of the recording period there is evidence of a jet at 2m on tower B, together w1th a signiflcant cross-slope component. By 2140, however, u was larger than $v$ at all levels. Around 2220 decreased and became falriy weak; it remalned so until approximately 0000 when $1 t$ started to pick up aga1n. The u component also increased around the same time so that the jet became less pronounced. Around 0100 the maximum in u appeared to rise to about $4 \mathrm{~m}$ and was slowly eroded so that 1 t was no longer apparent by the end of the data period.

\section{July 2-3 (Dars 183-184), 1981: 1813-0345 RST}

At 1600 PST on July 2 the surface weather chart for the northwest O.S. shows blgh pressure ridges along the continental divide and offshore WA. Between these ridges a warm trough extended from oR to southern wA and a weak frontal trough, also warm, reached to southern BC from the north. The Seattle-Hanford and Spokane-Hanford pressure differences were quite weak, $-0.9 \mathrm{mb}$ and $-1.4 \mathrm{mb}$ respectively. Pressure was falling over the ent1re state. A scattered ( $(5 / 10)$ layer of thin c1rrus cloud was noted at HMS, whlch Increased by 2200 to broken with a lower thin scattered layer. The thin clouds probably lnhlbited, but d1d not prevent, radiat1onal cooling after sunset. At the experimental site, skies were clearing at 2200 and were pretty well cleared by 2340 . By midnight the 1m temperature at HMS had cooled 13 deg $C$. Low-level winds were 11 ght, $1.5-3 \mathrm{~m} / \mathrm{s}$, over the hanford area and were generally from the north quadrant, varylng from NW at most telemetry sites in the northwest portion of the network to NoNE elsewhere.

By 2200 PST the Hanford area was under a pressure col with low pressure troughs north and south and h1gh pressure ridges east and west. Seattle-Hanford and Spokane-Hanford pressure differences had 1ncreased to $-2.1 m b$ and $-3.1 m b$, respect1vely. The two layers of thin c1rrus cloud observed at HMS covered $5 / 10$ to $7 / 10$ of the sky unt11 after $0400 \mathrm{Ju} 1 \mathrm{y} 3$, and then dissipated. 
Low-level winds were light throughout the period; at the Rattlesnake observatory, wind direction and speed were particularly constant from 2100 to 0400 at $1 \mathrm{~m} / \mathrm{s}$ from the $\mathrm{sw}$. At EOC the direction was more westerly, mostly about 280 degrees, with speeds of $3-4 \mathrm{~m} / \mathrm{s}$ near 2200 decreasing to $1.5 \mathrm{~m} / \mathrm{s}$ by 0900 and remalning less than $2 \mathrm{~m} / \mathrm{s}$ for the remalnder of the night.

By 0400 PST July 3 a narrow lobe of the coastal high pressure area extended Inland along the WA Cascades and 1nto central OR. The Rocky Mt. ridge of high pressure had moved little. A narrow trough of low pressure reached northward from NV through eastern WA to BC, where it jo1ned a weak cold front just north of the Wa/Canada border. A broader inverted trough lay along the OR coast. Seattle-Hanford and Spokane-Hanford pressure d1fferences were $-1.2 \mathrm{mb}$ and $-2.2 \mathrm{mb}$, a decrease since 2200. Low-1evel winds at the Prosser Barricade and EOC had veered more towards $N$ and $N W$, but speeds remalned $2 \mathrm{~m} / \mathrm{s}$ or less. Wind flow over the Hanford area at 0400 appears to define a microscale trough across the area from $N$ to SSE.

Eleven Tethersonde ascents were made this n1ght between 1950 and 0340. Prior to midnight the surface winds were from the W, shifting to NNW to $N$ above $50 \mathrm{~m}$. The ambient wind speed decreased from $4-5 \mathrm{~m} / \mathrm{s}$ at 2000 to 2-3 m/s after about 2120 , while the ambient potential temperature gradient Increased from less than 1 to $1.3 \mathrm{k} / \mathrm{km}$.

After midnight the layer below about $175 \mathrm{~m}$ cooled more rapidly than that above, forming a sharp inversion at that height. Winds below this helght were very light to calm, except in the surface katabatic layer where the wind direction shifted to WSW. The potential temperature gradient increased to an average of $3.4 \mathrm{~K} / \mathrm{km}$, becoming as large as $6 \mathrm{~K} / \mathrm{km}$ around 0130 when mixing temporarily eliminated the sharp inversion at 175m. The winds above $175 \mathrm{~m}$ remained from the $N$ at $2-3 \mathrm{~m} / \mathrm{s}$ unt1l about 0130 , after which they shifted to ENE at less than $2 \mathrm{~m} / \mathrm{s}$.

At tower B there was rapid cooling before 1900; afterward the 1m level cooled at $0.5 \mathrm{deg} \mathrm{C} / \mathrm{hr}$, but the $7.6 \mathrm{~m}$ level cooled at the smaller 


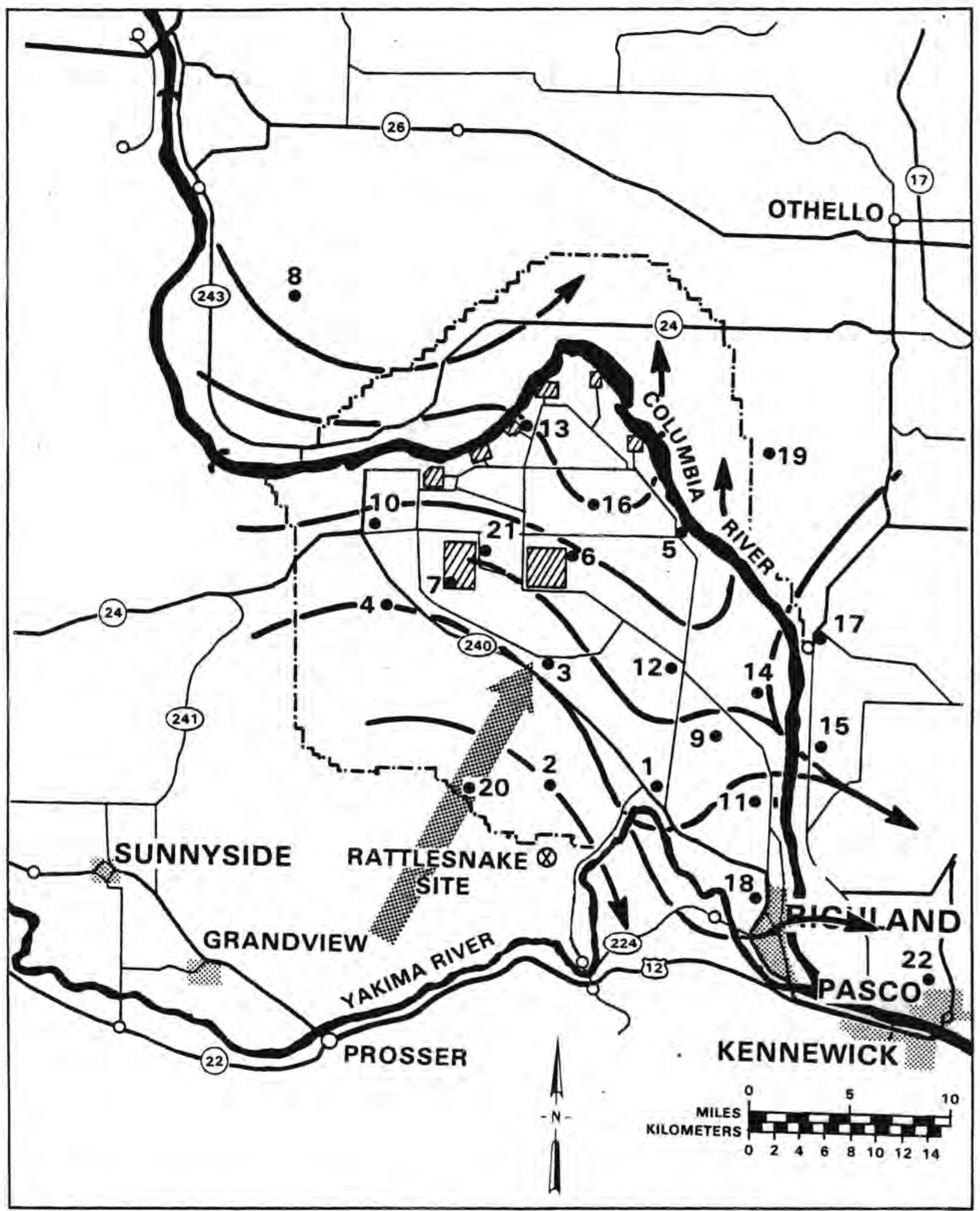

Figure 5. Hanford streamline analys1s, $2300 \mathrm{July} \mathrm{2,1981}$ 
rate of $0.3 \mathrm{deg} \mathrm{C} / \mathrm{hr}$. This cooling continued until midnight, when there was a sudden temperature drop of 2 deg $C$ in 30 minutes, after which the temperature was falrly constant. The inversion strength, $T(7.6 \mathrm{~m})-\mathrm{T}(1 \mathrm{~m})$, increased steadily from 1 deg C at 1900 to almost 3 deg C at 0200 , but with 15 minute fluctuations of almost 1 deg $C$. Early in the period, the winds at tower B were up-slope and cross-slope, but they soon became down-slope with a strong cross-slope component. There was evidence of a jet at $1.9 \mathrm{~m}$ on tower B. The downslope component weakened between 2000 and 2130 , but $v$ remained strong and the near-surface maximum in u persisted. Between 2130 and 2215 u 1 ncreased and then dropped again. Around 2330 the jet dropped to the $0.93 \mathrm{~m}$ level and remalned there. There was a problem with the data logger around 0230 , and when recording resumed around 0300 the jet was still at the lowest level. After 0230 the $v$ component was generally smaller than $u$, especially at the lower levels on the tower. In summary, there was good evidence of drainage winds during most of the night, with the cross-slope component gradually decreasing, especially after midnight.

October 16 (Day 289). 1981: 1510-2215 PST

At 1600 PST October 16 a large high pressure area was centered off the WA coast at $48 \mathrm{deg} N, 130 \mathrm{deg} W$. Pressure differences from Seattle and Spokane to Hanford were $-5.9 \mathrm{mb}$ and $-0.8 \mathrm{mb}$ respectively, and the pressure changes in WA had been slight during the previous 3 hours. A minor amount $(1 / 10$ to $2 / 10)$ of thin cirrus cloud was present, and even that disappeared after midnight. Radiational cooling began at sunset and the 1m temperature at HMS had cooled 12 deg C by 2200.

There were gaps in the EOC wind record during the evening hours; it appears that most of the time the wind was in the NW quadrant at less than $1 \mathrm{~m} / \mathrm{s}$. At the Prosser Barricade station the wind was mostiy in the $N$ H quadrant after 1900 (NE at $1 \mathrm{~m} / \mathrm{s}$ at 2300 ) until 0300 oct 17 . At the Rattlesnake Observatory the wind shifted gradually from sW to $N$ between 


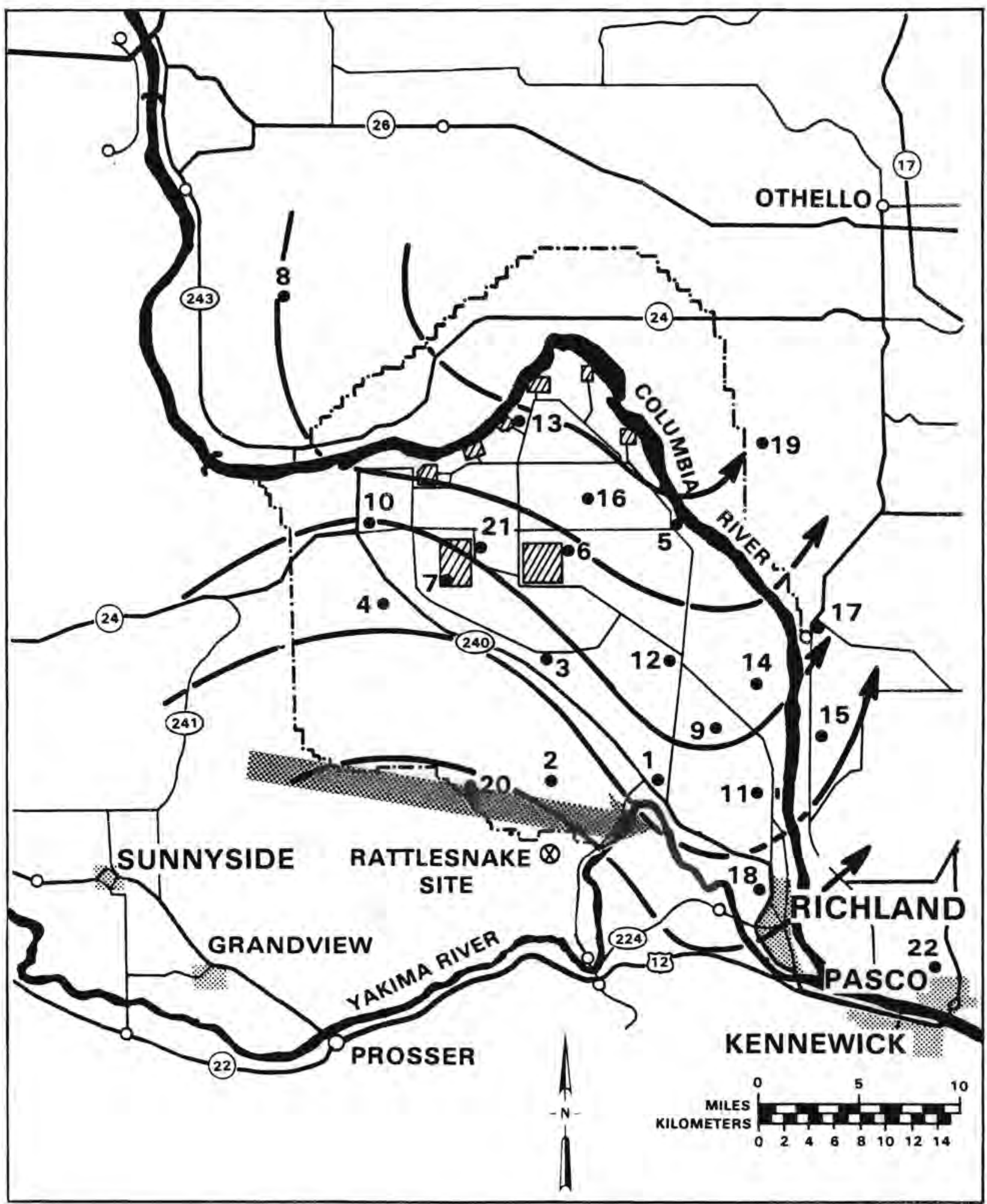

F1gure 6. Hanford streamline analysis, 2000 october 16, 1981 
1600 and 2300 and remalned from the $N$ the rest of the night. speeds over the lower portions of the mountaln were $7-9 \mathrm{~m} / \mathrm{s}$ after 2200 but less than 5 m/s prior to 2200. The local flow pattern was from the SE to E at 1600 , but by 1900 winds had shifted to westerly.

The surface high pressure area continued to bulld inland over northern WA and BC during the six hour period ending at 2200. The Seattle and Spokane to Hanford pressure differences were then $-4.4 m b$ and $+1.2 m b$. The sky over the Columbia Basin remalned clear the rest of the night. The pressure differences from seattle and spokane to Hanford continued to weaken as the surface high traveled eastward over the northen part of the state. Local wind flow became quite disorganized by 0400 PST on 0 ct 17 . As was mentioned, however, the wind over the crest of Rattlesnake Mt. shifted to more northerly and increased in speed.

Tethersonde ascents were made at 2000 and 2100 . The first showed a surface wind from WSW and westerly winds at $3-5 \mathrm{~m} / \mathrm{s}$ between $100 \mathrm{~m}$ and $250 \mathrm{~m}$. Winds above $250 \mathrm{~m}$ were from the $N$ and the potential temperature gradient was $2 \mathrm{~K} / \mathrm{km}$. The second ascent showed winds at all levels from NNW at $1-3 \mathrm{~m} / \mathrm{s}$.

At tower B there was some cooling from 1630 to 1800 , but from 1800 to 1930 the temperature Increased by 2 deg C and then cooled again by the same amount from 1930 to 2200. The Inversion strength, $T(9 \mathrm{II})-\mathrm{T}(2 \mathrm{~m})$, Increased from 0.5 deg $C$ at 1900 to 2 deg $C$ at 1945 , and then decreased to 0.5 to 1 deg C after 2030.

The perlod began with down-slope flow and some weak cross-slope flow. The downslope component was reasonably strong, but there was no evidence of drainage winds at tower B. Around 1630 the cross-slope component began to plck up. At 1830 u decreased and began to exh1b1t a jet with a maximum at $1.9 m$, but at 1930 u Increased and the jet rose to $3.2 m$ and became less pronounced. At 2015 u decreased again and the jet dropped to $1.9 m$ for the remalnder of the data record. 


\section{Tower Data}

The data collected in 1980 and 1981 and the summarles presented here were afflicted with a number of problems, inconsistencies and 1diosyncracies. Fortunately, most of these are minor and do not affect the usefulness of the data. In this section we will draw attention to known errors, and also present some supplementary information that may a1d in the interpretation and analysis of these data.

General comments

One of the more obvious differences in the 1980 and 1981 data summarles is the difference in format. There is no particular slgniflcance that should be attached to this; Instead, it was merely a matter of convenience to summarize the information in the manner shown and a matter of Inconvenience to so back and reduce everything to a common format.

In the tower data which follow, two separate time conventions are used, and they are related to the particular way in which the data were processed. For the 1980 tower data all times refer to the starting times of 10 minute averaging periods, while for the 1981 tower data the times are the ending times of 15 minute averaging periods. All times are Pacific Standard Time.

Tro features common to both the 1980 and 1981 data are:

Al I belghts IIsted are actualiy distances normal to the local slope, rather than vertical distances. The difference is negligible at tower C but is approximately 78 at towers $A$ and $B$.

The convention for wind components (listed in m/s) is that the $u$ component is positive when the wind is blowing downslope from 225 degrees, while the $v$ component is positive if the wind is blowing from 135 degrees. The wind vector has been rotated so that u is the component of the wind paraliel to the local slope while w is the component normal to the slope. A positive w points away from the surface. 
In several instances there are known or suspected errors in the data. When a known error has occurred, the data entries are replaced by a series of $9 \mathrm{~s}$, e.8., 9.99 or 999. When an error 1s suspected but the values are st1ll belleved to be indlcative of the general behavior of the atmosphere, a precautionary note is given here and the data are 11sted. Under such c1rcumstances, the data may be useful to indicate trends at a given location, but it would be unwse to use them for comparisons with similar quantitiés at other helghts. For example, one might use questionable data to estimate cooling rates at a given helght, but not for estimating Inversion strengths or Richardson numbers.

There are a number of points to be noted about the 1980 data in general, and these are followed by some specific comments about each of the 1980 cases. A similar procedure is then followed for the 1981 cases. 1980 Data

In 1980, the heights listed for tower $C$ are nominal helghts only. In fact, the wind and temperature-measurements were taken at slightly different elevations as shown in table 4 below.

Table 4. 1980 Instrument heights (m) for tower C

\section{Nominal Helght Temperature Height Hind Soeed Height}

$17 \cdot 3$

9.8

6.0

2.7

1.4
16.6

9.4

$5 \cdot 4$

$2 \cdot 5$

1.4
$17 \cdot 5$

9.9

6.1

2. 7

1.4

The depths at which soll temperatures were recorded were 1,3 and 7 cm, reading from left to right on the computer summaries.

The angle PHI listed under the data for towers B and $C$ is the angle that the net downslope component of the wind makes with the horizontal. Th1s was calculated before the wind components were rotated into the plane of the local slope. Hence, if the wind were truly parallel to the slope, 


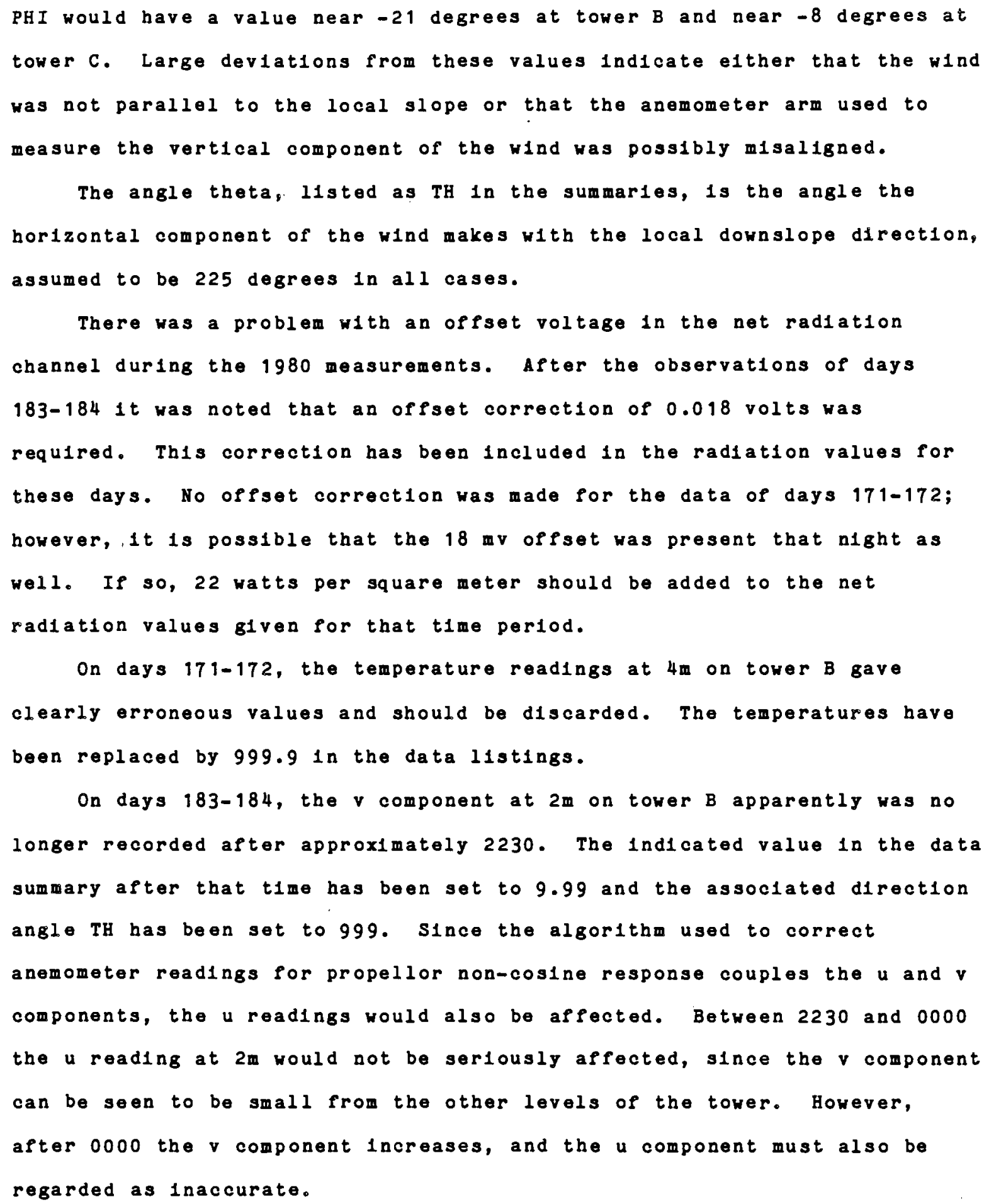


1981 Data

Soll temperatures were not recovered in the 1981 cases and are, therefore, not listed In the data summaries. The angle DIR is simply the direction of the horizontal wind component, with the downslope direction being approximately 225 degrees.

The anemometry on tower A was often temperamental. It appears that the speed and direction values at $10.65 \mathrm{~m}$ and $3.16 \mathrm{~m}$ are $1 \mathrm{n}$ error, and the values at $1.37 \mathrm{~m}$ are open to question as well. For the $10.65 \mathrm{~m}$ and $3.16 \mathrm{~m}$ levels, values of 9.99 have been assigned for the u and $v$ components, and the direction values have been set to 999.

For days 183-184, the temperature at $0.87 \mathrm{~m}$ on tower A $1 \mathrm{~s}$ not rellable and may be in error by a degree or so. Field notes show that the aspirators at $13.75 \mathrm{~m}$ on tower $B$ and $0.31 \mathrm{~m}$ on tower $C$ were not working when they were checked about two weeks later. Hence, the temperatures recorded at these levels must be regarded as suspect as well. This seems to be especially true for the $13.75 \mathrm{~m}$ level on tower B, which is inconsistent with the remalnder of the temperature profile.

The net radiation measurements on these days also look peculiar, primarliy because of thelr remarkable constancy. We have no evidence that there was actualiy a malfunction in the equipment, but 1 t may be worth noting this rather odd feature.

For day 289 , the temperature at the $0.95 \mathrm{~m}$ level on tower $B$ is in error, and has been set to 999. The net radiation measurements also falled during this measurement period. The vertical component of the anemometer at the $3.21 \mathrm{~m}$ level on tower $B$ gave a zero reading during this time, but since the wind component normal to the slope (w) is largely a result of the horizontal wind projected on the normal axis, the resultant error should be small. 
JUHE 19-20, 1980; 1841-0251 PST

TOWER A

DAY ITI STARTING TIME 1A840840

$\begin{array}{llll}5.8 & 301.3 & 9.1 & 301.7 \\ 3.8 & 301.1 & 6.4 & 301.4 \\ 2.0 & 300.8 & 4.0 & 999.9 \\ 1.1 & 300.4 & 2.0 & 301.2 \\ & 1.2 & 301.0\end{array}$

SOIL TEMPERATURES 301.0

DAY III STARTING TIME 18850848

$5.8301 .3 \quad 9.1301 .7$

$\begin{array}{llll}3.8 & 301.0 & 6.4 & 301.4 \\ 2.0 & 300.7 & 9.0 & 999.9\end{array}$

1.1300 .2

2.0301 .0

SOIL TEMPERATURES : $\begin{array}{ccc}30.2 & 30.6 & -1.21 \\ 30.3\end{array}$

IONE $R$ B

OAY ITI STARTING TIME 198 O\&4O

$\begin{array}{ll}5.8 & 301.2 \\ 3.8 & 300.9 \\ 2.0 & 300.4 \\ 1.1 & 299.9\end{array}$

9.1301 .5

6.4
4.0 999.9

2.0300 .8

TEMPERATURE 3 :

29.7

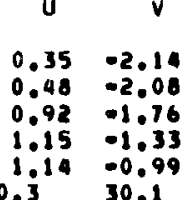

$\begin{array}{ccccc}W & T H & \text { PHI } & \text { HT } & \text { POT I } \\ 0.33 & -96 . & 99 . & 17.3 & 301.5 \\ 0.01 & -90 . & 70 . & 9.8 & 301.2 \\ 0.21 & -75 . & 5 . & 6.0 & 300.7 \\ 0.27 & -62 . & 0 . & 2.7 & 300.4 \\ 0.21 & -56 . & -4 . & 1.4 & 300.0\end{array}$

DAY ITI STARTING TIME 19110849
HT POT T MOT T

$\begin{array}{ll}5.8 & 301.1 \\ 3.8 & 300.1 \\ 2.0 & 300.2\end{array}$

$\begin{array}{ll}2.0 & 300.2 \\ 1.1 & 299.6\end{array}$

$\begin{array}{ll}9.1 & 301.5 \\ 6.4 & 301.2 \\ 4.0 & 999.9 \\ 2.0 & 300.6 \\ 1.2 & 300.1\end{array}$

SOIL TEMPERATURES 29.2

.330 .1

H TH PHI HT POT T

$\begin{array}{lllll}0.50 & -83 . & 38 . & 17.3 & 301.7 \\ 0.53 & -80 . & 30 . & 9.8 & 308.4\end{array}$

$0.21 \quad-67 . \quad-6 . \quad 6.0300 .8$

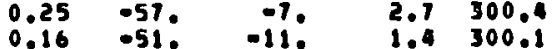

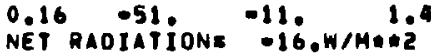

W TH PHI HT POT

$0.45 \quad-81 . \quad 32 . \quad 17.3301 .7$

$0.06 \quad-77.0 \quad 23 . \quad 9.8301 .9$

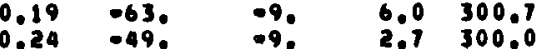

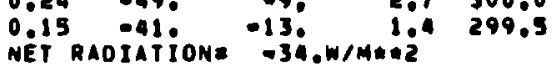

$\begin{array}{ccccc}U & V & W & T H & P H I \\ 0.10 & -1.80 & 0.30 & -87 . & 63 . \\ 1.14 & -1.47 & 0.11 & -52 . & -3 . \\ 1.39 & -1.46 & 0.46 & -46 . & 10 . \\ 1.41 & -0.92 & 0.44 & -33 . & 9 . \\ 1.29 & 0.05 & -0.23 & 2 . & -18 .\end{array}$

OAY 171 STARTING TIME 19820849

$\begin{array}{ll}0.62 & -2.12 \\ 0.73 & -2.08 \\ 1.20 & -1.74 \\ 1.51 & -1.20 \\ 1.48 & -0.80 \\ 29.9 & 29.9\end{array}$

W TH PHI HT POT T

$0.47 \quad-74 . \quad 17 . \quad 17.3 \quad 301.8$

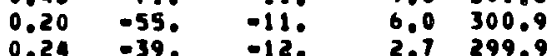

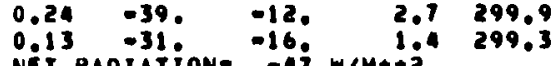
NET RAOIATION= -16: WIMEIA

$\begin{array}{ccccc}U & V & W & T H & P H 1 \\ 1.03 & -2.54 & 0.40 & -60 . & 17 . \\ 1.57 & -2.24 & 0.19 & -55 & -1 . \\ 1.53 & -2.02 & 0.55 & -53 . & 11 . \\ 1.29 & -1.37 & 0.47 & -47 & 12 . \\ 1.15 & -0.20 & -0.47 & -13 & -31 .\end{array}$

$\begin{array}{llll}5.8 & 301.1 & 9.1 & 301.6 \\ 3.8 & 300.8 & 6.4 & 301.3 \\ 2.0 & 300.1 & 4.0 & 999.9 \\ 1.1 & 299.5 & 2.0 & 300.5\end{array}$

$\begin{array}{ll}3.0 & 300.1 \\ 1.1 & 299.5\end{array}$

2.0300 .5

SOIL TEMPEHATURESI

299.9

DAY 171 STARTING TIME 19130249

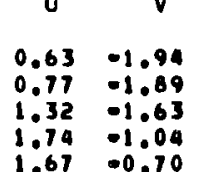

W TH PHI HT POT T

$\begin{array}{ccccc}1 & V & W & T H & \text { PHI } \\ 0.93 & -2.29 & 0.33 & -68 . & 11 . \\ 1.65 & -2.21 & 0.08 & -53 . & -5 . \\ 1.80 & -2.06 & 0.09 & -48 . & 6.06 \\ 1.80 & -1.25 & 0.11 & -35 . & 5 . \\ 1.57 & -0.11 & -0.31 & -5 . & -20 .\end{array}$ $\begin{array}{lllll}1.18 & -2.32 & 0.35 & -63 . & 8 . \\ 1.85 & -2.24 & 0.06 & -50 . & -6 . \\ 2.11 & -2.11 & 0.44 & -45 . & 4 . \\ 2.09 & -1.22 & 0.36 & -31 . & 2 . \\ 1.73 & -0.11 & -0.28 & -4 . & -17 .\end{array}$

$\begin{array}{ccccc}W & T H & \text { PHI } & \text { HT } & \text { POT T } \\ 0.51 & -72 . & 18 . & 17.3 & 301.8 \\ 0.446 & -68 . & 10 . & 9.8 & 301.6 \\ 0.19 & -51 . & -13 . & 6.0 & 300.9 \\ 0.26 & -31 . & -12 . & 2.7 & 299.5 \\ 0.14 & -233 & -16 . & 1.4 & 298.8\end{array}$

$\begin{array}{ccccr}U & V & W & T H & P H I \\ 1.27 & -2.26 & 0.37 & -61 . & 0 . \\ 1.80 & -2.15 & 0.07 & -49 . & -6.0 \\ 2.18 & -2.03 & 0.49 & -43 . & 40 \\ 2.14 & -1.110 & 0.38 & -27 . & 2 . \\ 1.78 & -0.05 & -0.21 & -2 . & -150\end{array}$

\begin{tabular}{|c|c|c|c|c|c|c|c|c|c|c|}
\hline TT & POT & HT & POT T & U & $v$ & $w$ & TH & PHI & HT & POT T \\
\hline $\begin{array}{l}5.8 \\
3.8 \\
2.0 \\
1.1\end{array}$ & $\begin{array}{l}301.0 \\
300.7 \\
300.0 \\
249.3\end{array}$ & $\begin{array}{l}9.1 \\
6.4 \\
4.0 \\
2.0 \\
1.2\end{array}$ & $\begin{array}{l}301.5 \\
301.2 \\
999.9 \\
300.2 \\
299.6 \\
28.2\end{array}$ & $\begin{array}{l}0.77 \\
0.91 \\
1.54 \\
1.95 \\
1.85 \\
29.2\end{array}$ & $\begin{array}{l}-2.32 \\
-2.30 \\
-1.94 \\
-1.20 \\
-0.00 \\
29.4\end{array}$ & $\begin{array}{l}0.53 \\
0.48 \\
0.19 \\
0.27 \\
0.12 \\
N E T\end{array}$ & $\begin{array}{l}-72 . \\
-680 \\
-520 \\
-320 \\
-23 \\
\text { DIATION= }\end{array}$ & $\begin{array}{r}140 \\
70 \\
-140 \\
-130 \\
-17 . \\
-56 .\end{array}$ & $\begin{array}{r}17.3 \\
9.8 \\
6.0 \\
2.7 \\
1.4 \\
4=1.2\end{array}$ & $\begin{array}{l}301.8 \\
301.5 \\
300.6 \\
299.2 \\
298.6\end{array}$ \\
\hline
\end{tabular}

$\begin{array}{ccccr}U & V & W & T H & P H I \\ 1.33 & -2.60 & 0.43 & -63 . & 9 . \\ 2.12 & -2.44 & 0.05 & -49 . & -7 . \\ 2.48 & -2.20 & 0.50 & -42 . & 3 . \\ 2.33 & -1.19 & 0.32 & -27 . & -0 . \\ 1.92 & -0.10 & -0.24 & -3 . & -15 .\end{array}$ 
JUNE 19-20, 1980; 1841-0251 PST

DAY TOWER A 171 SIARTING IIATE 19:40149

TOWEN $B$

TONER C

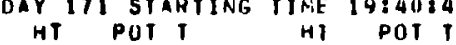

$$
\begin{array}{ll}
5.8 & 300.9 \\
3.8 & 300.6 \\
2.0 & 299.9 \\
1.1 & 299.3
\end{array}
$$

9.1301 .5

$\begin{array}{llll}9.1 & 301.5 & 0.92 & -2.49 \\ 6.4 & 301.2 & 1.13 & -2.45 \\ 4.0 & 999.9 & 1.70 & -1.98 \\ 2.0 & 300.1 & 2.01 & -1.21\end{array}$

$2.6 \quad 300.1$

$1.70-1.98$

SOIL TEMPERATURES:

$\begin{array}{ccc}299.5 & 1.86 & -1.21 \\ 21.8 & 28.9 & 29.83\end{array}$

DAY 171 STARTING TIME 19850849

HT POT T HT POT T

$\begin{array}{ll}5.8 & 301.0 \\ 3.8 & 300.7\end{array}$

2.0300 .0

$\begin{array}{ll}9.1 & 301.6 \\ 6.4 & 301.3 \\ 4.0 & 999.9 \\ 2.0 & 300.3 \\ 1.2 & 299.6\end{array}$

$11 v$

$\begin{array}{ll}W & T H \\ 0.52 & -70 . \\ 0.46 & -65 \\ 0.22 & -49 \\ 0.28 & -31: \\ 0.12 & -24: \\ \text { NET HADIATION }\end{array}$

PHI

HT POT T

$u\left(\frac{1}{2}\right)$

SOIL TEMPERATURES:

1.2299 .6

$0.93-2.13$

$1.93-1.12$

DAY 171 STARTING TIME 2020149

$\begin{array}{ll}1.85 & -0.73 \\ 0.6 & 29.0\end{array}$

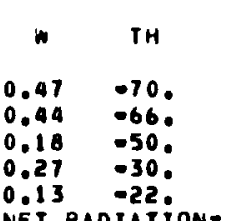

9. $17.3 \quad 301.8$

1. $\quad 9.8301 .5$

$-13 . \quad 2.7299 .3$

$\begin{array}{lll}1.51 & -2.75 & 0.40\end{array}$

$2.32 \quad-2.56 \quad 0.06$

$2.64 \quad-2.22 \quad 0.44$

$\begin{array}{rrrrr}2.64 & -2.22 & 0.44 & -40 . & 10 \\ 2.36 & -1.23 & 0.35 & -28 . & 0 . \\ 1.92 & -0.11 & -0.25 & -3 . & -16 .\end{array}$

HT POT T HT POT T

$\begin{array}{llllll}5.8 & 301.1 & 9.1 & 301.5 & 0.29 & -1.96 \\ 3.8 & 300.7 & 6.4 & 301.3 & 0.40 & -1.93 \\ 2.0 & 299.9 & 4.0 & 999.9 & 0.94 & -1.67 \\ 1.1 & 299.2 & 2.0 & 300.6 & 1.41 & -1.20 \\ & & 1.2 & 299.8 & 1.51 & -0.82\end{array}$

¿ SOIL TEMPERATURES:

$\begin{array}{cc}1.41 & -1.20 \\ 28.21 & -0.82 \\ 28.8 & \end{array}$

DAY 171 STARTING. TIME 20810150

HT POT T HT POT

$$
\begin{array}{ll}
5.8 & 300.9 \\
3.8 & 300.6 \\
2.0 & 299.7 \\
1.1 & 298.9
\end{array}
$$

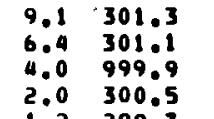

$0.17-1.92$

$0.30-1.92$

SOIL TEMPERATURESI $299.7 \quad 1.48 \quad 00.82$

DAY ITI STARTING TIME 20820150
HT POT T POT T U U

$27.9^{1.48} \quad \begin{array}{cc}-0.82 \\ 28.6\end{array}$

NET RADIATION= -64.WIMHEL

$\begin{array}{rrr}\text { I } & \text { HT } & \text { POT T } \\ \text { 10. } & 17.3 & 301.8 \\ 4 . & 9.8 & 301.6 \\ 4 . & 6.0 & 300.8 \\ 3: & 2.7 & 299.3 \\ 7 . & 1.4 & 290.6\end{array}$

$\begin{array}{ccccr}U & V & W & T H & \text { PHI } \\ 1.43 & -2.39 & 0.39 & -59 . & 7 . \\ 2.13 & -2.22 & 0.06 & -46: & -7 . \\ 2.41 & -1.97 & 0.49 & -39 . & 3 . \\ 2.24 & -1.06 & 0.36 & -25: & 1 . \\ 1.63 & -0.02 & -0.18 & -1 . & -14 .\end{array}$

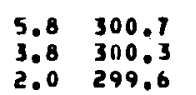

2.0299 .6

$9.1 \quad 301.2 \quad-0.04 \quad-2.15$ $\begin{array}{llll}6.4 & 300.9 & 0.20 & -2.110 \\ 4.0 & 999.9 & 0.80 & -2.23\end{array}$ $2.0300 .3 \quad 1.18 \quad-1.69$

SOIL TEMPERATURES

$\begin{array}{lll}299.8 & 1.19 & -1.21 \\ 26.3 & 27.6 & 28.3\end{array}$

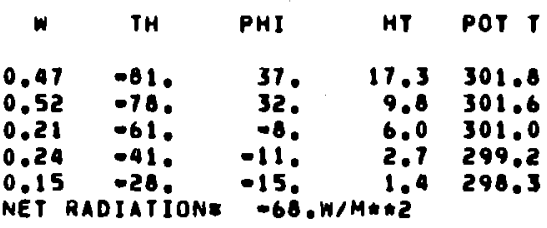

$u$

$\begin{array}{lllll}0.95 & -2.23 & 0.36 & -670 & 12 .\end{array}$

$\begin{array}{lllll}2.09 & -2.00 & 0.54 & -440 & 6 . \\ 2.26 & -1.06 & 0.38 & -25 & 1 .\end{array}$

$\begin{array}{rrrrr}2.26 & -1.06 & 0.38 & -25 . & 1 \\ 1.86 & 0.05 & -0.14 & 2 . & -13 .\end{array}$

OAY 111 STARTING TIME 20830150

$\begin{array}{lrrrr}W & T H & \text { PHI } & \text { HT } & \text { POT T } \\ 0.45 & -85 . & 99 . & 17.3 & 301.6 \\ 0.52 & -81 . & 39 . & 9.8 & 301.4 \\ 0.19 & -62 . & -8 . & 6.0 & 300.9 \\ 0.21 & -42 . & -12 . & 2.7 & 299.1 \\ 0.15 & -29 . & -15 . & 1.4 & 297.7\end{array}$

$\begin{array}{ccccr}U & V & W & T H & P H I \\ 0.87 & -2.08 & 0.32 & -61 . & 12 . \\ 1.61 & -1.90 & 0.06 & -50 . & -6 . \\ 1.89 & -1.85 & 0.54 & -44 . & 8 . \\ 2.20 & -1.02 & 0.42 & -25 . & 2 . \\ 1.92 & 0.15 & -0.10 & 5 . & -11 .\end{array}$

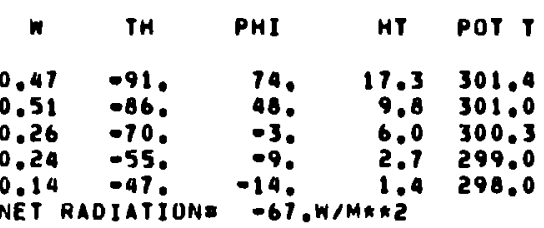

$\begin{array}{lllllll}0.61 & -2.17 & 0.37 \quad-77, \quad 23 .\end{array}$

\begin{tabular}{|c|c|c|c|c|}
\hline$w$ & TH & PHI & HT & POT \\
\hline $\begin{array}{l}0.41 \\
0.51 \\
0.26 \\
0.24 \\
0.14\end{array}$ & $\begin{array}{l}-92 . \\
-87 . \\
-69 . \\
-52 . \\
-42\end{array}$ & $\begin{array}{l}60 . \\
54 . \\
-3 . \\
-10 . \\
-14 .\end{array}$ & $\begin{array}{r}17.3 \\
9.4 \\
6.0 \\
2.7 \\
1.9\end{array}$ & $\begin{array}{l}301.3 \\
301.0 \\
300.2 \\
298.8 \\
297.9\end{array}$ \\
\hline
\end{tabular}

$\begin{array}{lll}0.78 & -2.45 & 0.06\end{array}$ 2.150 .020 .04 $\begin{array}{lll}1.26 & 0.25 & 0.31\end{array}$ NET RADIATION= -67 ,W/MKK2

$\begin{array}{llllll}\text { HT POT T } & \text { HT } & \text { POT T } & U & V \\ 5.8 & 300.1 & 9.1 & 301.1 & -0.08 & -2.47 \\ 3.8 & 300.3 & 6.4 & 300.8 & 0.14 & -2.41 \\ 2.0 & 299.6 & 4.0 & 999.9 & 0.79 & -2.06 \\ 1.1 & 298.9 & 2.0 & 300.1 & 1.21 & -1.55 \\ & & 1.2 & 299.5 & 1.25 & -1.14 \\ \text { SOIL TEMPERATURES } & & 25.9 & 27.3 & 28.1\end{array}$

$\begin{array}{ccccr}U & V & W & T H & P H I \\ 0.46 & -2.56 & 0.36 & -80 . & 32 . \\ 1.56 & -2.41 & 0.06 & -57 . & -6 . \\ 2.03 & -2.33 & 0.50 & -49 . & 6 . \\ 2.17 & -1.28 & 0.37 & -31 . & 2 . \\ 1.84 & -0.11 & -0.25 & -3 . & -16 .\end{array}$


JUNE 19-20, 1980; 1841-0251 PST

TONEK ITI STAHTING TIME 208408 BO

TINES H

TOWER C

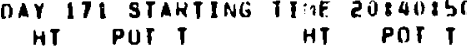

$\begin{array}{ll}5.8 & 300.5 \\ 3.8 & 300.1 \\ 2.0 & 290.3\end{array}$

$\begin{array}{ll}2.0 & 299.3 \\ 1.1 & 296.7\end{array}$

$\begin{array}{llll}9.1 & 3110.9 & 0.40 & -3.23 \\ 6.4 & 310.6 & 0.62 & -3.09\end{array}$

$4.0 \quad 499.9 \quad 1.25 \quad-2.62$

SOIL TEMPERATURES

$4.0299 .8 \quad 1.48$

$\begin{array}{lll}299.2 & 1.39 & -1.43 \\ 25.6 & 27.0 & 27.9\end{array}$

dar 171 STARTING TIME 20850150

HT POT T HI POT

$\begin{array}{ll}5.8 & 300.5 \\ 3.8 & 300.1\end{array}$

$\begin{array}{ll}3.8 & 300.1 \\ 2.0 & 299.3\end{array}$

9.1300 .9

$\begin{array}{lll}0.32 & -2.90\end{array}$

$\begin{array}{llll}4.0 & 999.9 & 1.15 & -2.41 \\ 2.0 & 299.9 & 1.42 & -1.71\end{array}$

$1.0 \quad 299.9 \quad 1.42 \quad-1.7$

SOIL TEMPEHATURES:

299.3
25.2

$\begin{array}{ll}1.0^{2} & -1.39 \\ & 27.7\end{array}$

DAY 171 SIARIING TIME 211 OISO

$\begin{array}{ll}5.0 & 300.5 \\ 3.8 & 300.0\end{array}$

$\begin{array}{ll}2.0 & 299.2 \\ 1.1 & 298.6\end{array}$

$\approx$

SOIL TEMPEAATURE $8:$

9.1300 .8

$6.4 \quad 300.5$

$\begin{array}{ll}4.0 & 999.9 \\ 2.0 & 299.6\end{array}$

$\begin{array}{ll}0.50 & -2.81 \\ 1.14 & -2.38\end{array}$

$1.48-1.75$

$\begin{array}{ccc}299.0 & 1.42 & -1.32 \\ 24.9 & 26.5 & 27.5\end{array}$

DAY 171 SIAHTING TIME 21110151

HT POT T HT POT

$\begin{array}{ll}5.8 & 300.2 \\ 3.8 & 299.8\end{array}$

$\begin{array}{ll}3.8 & 299.8 \\ 2.0 & 299.1\end{array}$

$\begin{array}{ll}9.1 & 300.6 \\ 6.4 & 300.3 \\ 4.0 & 999.9 \\ 2.0 & 299.6\end{array}$

1.1298 .5

SOIL TEMPERATURES

1.2299 .0

DAY ITI STARTING TIME 21220151

$$
\begin{array}{ll}
5.8 & 299.8 \\
3.8 & 299.4 \\
2.0 & 298.7 \\
1.1 & 298.2
\end{array}
$$

SOIL TEMPERATURES:

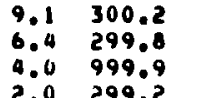

$1.10-3.26$

$\begin{array}{llll}2.0 & 299.2 & 1.30 & -2.03\end{array}$

\begin{tabular}{|c|c|}
\hline $\begin{array}{l}0.53 \\
0.58 \\
0.18 \\
0.22 \\
0.10 \\
\text { NET HAD }\end{array}$ & $\begin{array}{l}-43 . \\
-79 . \\
-640 \\
-520 \\
-46 . \\
D I A T I O N=\end{array}$ \\
\hline
\end{tabular}

$\begin{array}{lll}24.3 & 25.9^{1.20} & -1.59 \\ 27.0 & \end{array}$

PHI

W TH PHI

$0.47-84$.

0.18 -65.

6.0299 .6

.11 -44. -16.01 .4297 .9$

POT T

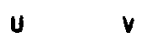

DAY 171 STAKTING TIME 21830851

MT POT I

$\begin{array}{ll}5.8 & 299.6 \\ 3.8 & 299.3 \\ 2.0 & 298.1\end{array}$

2.0298.

$\begin{array}{ll}9.1 & 300.0 \\ 6.4 & 299.7 \\ 4.0 & 999.9 \\ 2.0 & 299.1\end{array}$

$0.60 \quad-3.57$

$1.77-3.39$

$1.48-2.16$

SOIL IEMPERATURES:

\begin{tabular}{|c|c|c|c|c|}
\hline w & TH & PHI & HT & POT 1 \\
\hline $\begin{array}{l}0.51 \\
0.55 \\
0.20 \\
0.23 \\
0.12 \\
\text { NET RAD }\end{array}$ & $\begin{array}{l}-84 . \\
-80 . \\
-64 . \\
-50 . \\
-43 \\
\text { ITATION= }\end{array}$ & $\begin{array}{r}30 . \\
27 . \\
-11: \\
-12 . \\
-16 . \\
-15 .\end{array}$ & $\begin{array}{r}17.3 \\
9.8 \\
6.0 \\
2.7 \\
1.4 \\
1.9\end{array}$ & $\begin{array}{l}301.1 \\
300.1 \\
299.6 \\
298.2 \\
297.5\end{array}$ \\
\hline$w$ & TH & PHI & HT & Por $T$ \\
\hline $\begin{array}{l}0.48 \\
0.35 \\
0.24 \\
0.25 \\
0.13\end{array}$ & $\begin{array}{l}-92 . \\
-88 . \\
-730 \\
-59 . \\
-51 .\end{array}$ & $\begin{array}{l}84 . \\
61 . \\
-30 \\
-8 . \\
-14 .\end{array}$ & $\begin{array}{r}17.3 \\
9.8 \\
6.0 \\
2.7 \\
1.4\end{array}$ & $\begin{array}{l}300.9 \\
300.4 \\
299.4 \\
298.3 \\
297.6\end{array}$ \\
\hline
\end{tabular}

$1.37-1.69$

$\begin{array}{lll}0.97 & -3.37 & 0.42 \\ 2.17 & -3.16 & 0.01\end{array}$

$2.61-2.46 \quad 0.43$

$2.43 \quad-1.61 \quad 0.33$

$1.90-0.21-0.36$

$-74 . \quad 15$.

$-33 . \quad-0$.

13. $\quad 6.0299 .6$

4297.9

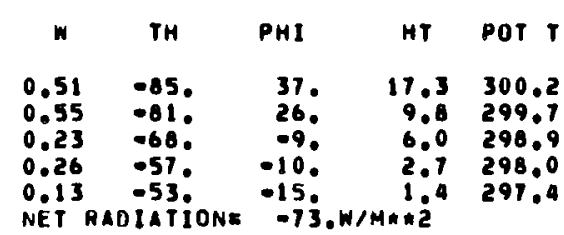

$\begin{array}{ccccr}U & V & W & T H & P H I \\ 1.07 & -3.04 & 0.42 & -71 . & 13 . \\ 2.06 & -2.88 & 0.05 & -54 . & -7 . \\ 2.45 & -2.62 & 0.45 & -47 . & 2 . \\ 2.33 & -1.49 & 0.33 & -33 . & -0 . \\ 1.91 & -0.10 & -0.33 & -5 . & -18 .\end{array}$

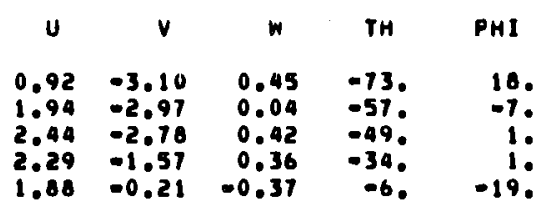

$\begin{array}{ccccr}U & V & W & \text { TH } & \text { PHI } \\ 0.36 & -3.29 & 0.36 & -84 . & 37 . \\ 1.59 & -2.93 & 0.02 & -62 . & -8 . \\ 2.07 & -2.81 & 0.44 & -54 . & 4 . \\ 2.07 & -1.76 & 0.35 & -40 . & 1 \\ 1.80 & -0.29 & -0.47 & -9 . & -23 .\end{array}$

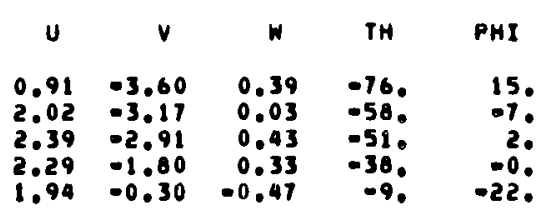

W TH PHI HT POT T

$\begin{array}{rrrrr}0.49 & -81 . & 19 . & 17.3 & 300.8 \\ 0.48 & -71 . & 11 . & 9.8 & 299.7 \\ 0.18 & -65 & -13 & 6.0 & 298.7 .\end{array}$

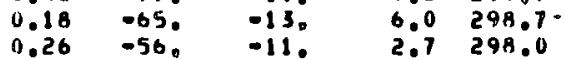

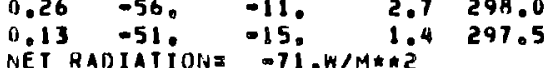

$\begin{array}{ccccr}U & V & W & T H & P H I \\ 1.43 & -3.82 & 0.36 & -70 . & 6 . \\ 2.35 & -3.28 & 0.03 & -54 . & -8 . \\ 2.56 & -2.89 & 0.40 & -48 . & 1 . \\ 2.34 & -1.03 & 0.32 & -38 . & -1 . \\ 1.95 & -0.33 & -0.50 & -100 & -23 .\end{array}$


JUNE 19-20, 1980; 1841-0251 PST

DAY IONER SÁ

HI POT TING TIME 2184085

$5.8294 .6 \quad 9.1300 .0$

$\begin{array}{llll}3.8 & 299.3 & \\ 2.0 & 298.7 & 6.4 & 299.7\end{array}$

1.1298 .2

$\begin{array}{ll}4.0 & 999.9 \\ 2.0 & 299.1\end{array}$

$1.19 \quad-2.65$

SOIL TEMPERATURES:

$\begin{array}{rrr}298.8 & 1.37 & -2.00 \\ 23 & 1.27 & -1.56\end{array}$

DAY ITI STARING TIME 21350151

$\begin{array}{ll}1.27 & -1.56 \\ 5.5 & 26.6\end{array}$
TOWEA B

$\begin{array}{ccccc}W & \text { TH } & \text { PHI } & \text { HT } & \text { POT T } \\ 0.51 & -82 . & 25 . & 17.3 & 300.1 \\ 0.52 & -78, & 17 . & 9.8 & 299.7 \\ 0.24 & -66 . & -10 . & 6.0 & 298.9 \\ 0.28 & -56 . & -9 . & 2.7 & 298.0 \\ 0.15 & -51 . & -14 . & 1.4 & 297.5\end{array}$

TOWER C

$\begin{array}{ccccr}U & V & W & \text { TH } & \text { PHI } \\ 1.13 & -3.56 & 0.39 & -72 . & 11 . \\ 2.15 & -3.11 & 0.02 & -55 . & -8 . \\ 2.39 & -2.76 & 0.44 & -49 . & 2 . \\ 2.23 & -1.70 & 0.34 & -37 . & 11 \\ 1.91 & -0.27 & -0.43 & -8 . & -21 .\end{array}$

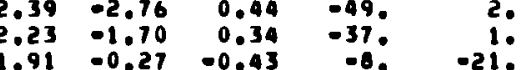

DAT 171 STARTING TIME 21350151

$\begin{array}{ll}5.8 & 299.8 \\ 3.8 & 299.5 \\ 2.0 & 298.9 \\ 1.1 & 298.5\end{array}$

9.1300 .1

$\begin{array}{ll}6.4 & 299.8 \\ 4.0 & 999.9\end{array}$ $2.0 \quad 299.2$

1.1298 298.8

SOIL TEMPERATURES 3.7

DAY ITI STARTING TIME 22,0151

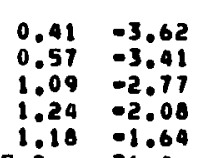

w TH PHI

HT POT T

$0.57 \quad-84 . \quad 34 . \quad 17.3 \quad 300.3$

$\begin{array}{lllll}0.58 & -81 . & 24 . & 9.8 & 299.8 \\ 0.22 & -69 . & -9 . & 6.0 & 298.9\end{array}$

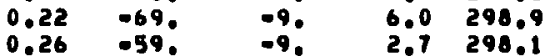

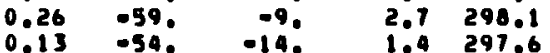
NET RADIATION= $-14.68 . \mathrm{W} / \mathrm{MANZ}$

$\begin{array}{ccccr}U & V & W & T H & \text { PHI } \\ 1.16 & -3.80 & 0.39 & -13 . & 10 . \\ 2.16 & -3.32 & 0.02 & -57 . & -8 . \\ 2.31 & -2.95 & 0.42 & -52 . & 2 . \\ 2.10 & -1.89 & 0.35 & -42 . & 1 \% \\ 1.82 & -0.34 & -0.52 & -11 . & -24 .\end{array}$
$\begin{array}{rrrr}2.31 & -2.95 & 0.42 & -52 \\ 2.10 & -1.89 & 0.35 & -42 \\ 1.82 & -0.34 & -0.52 & -11\end{array}$

$-24$.

HT POT $T$ HT POT $T$

$\begin{array}{ll}5.8 & 299.7 \\ 3.8 & 299.3\end{array}$

2.0298 .1

9.1300 .1

4.0999 .9

$\begin{array}{rl}4.0 & 299.2\end{array}$

SOIL TEMPERATURES

23.5

\section{u v}

W TH PHI HT POT $P$

$0.55 \quad-01 . \quad 25 . \quad 17.3 \quad 300.3$

$0.55 \quad-70 . \quad 18.09 .8299 .9$ $0.18 \quad-66 . \quad-12 . \quad 6.0298 .9$ 0.09 -51: -17: 1.4297 .3 NET RADIATIONE - 68. W/MME

DAY ITI STARTING TIME 22810152
HT POT T

$\begin{array}{llllll}5.8 & 299.8 & 9.1 & 300.1 & 0.26 & -3.52 \\ 3.8 & 299.4 & 6.4 & 299.8 & 0.47 & -3.32 \\ 2.0 & 298.8 & 4.0 & 999.9 & 1.03 & -2.76 \\ 1.1 & 298.3 & 2.0 & 299.2 & 1.24 & -2.10 \\ & & 1.2 & 296.8 & 1.20 & -1.64\end{array}$

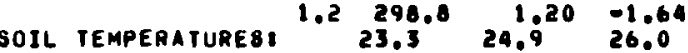

$n$ TH PHI HT POT T

$\begin{array}{lllll}0.52 & -86 . & 43 . & 17.3 & 300.4 \\ 0.52 & -82 . & 27 . & 9.8 & 299.8\end{array}$

$\begin{array}{lllll}0.52 & -82 . & 27 . & 9.8 & 299.8 \\ 0.21 & -70 . & -9 . & 6.0 & 298.8\end{array}$

$\begin{array}{lllll}0.21 & -70 . & -9 . & 6.0 & 298.8 \\ 0.25 & -59 . & -10 . & 2.7 & 297.9\end{array}$

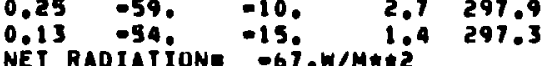

DAY 171 STARTING TIME $22: 20152$

$\begin{array}{lllllc}\text { HT } & \text { POT T } & \text { HT } & \text { POT T } & \text { U } & V \\ 5.8 & 299.7 & 9.1 & 300.2 & 0.29 & -3.39 \\ 3.8 & 299.3 & 6.4 & 299.8 & 0.49 & -3.24 \\ 2.0 & 298.8 & 4.0 & 999.9 & 1.04 & -2.67 \\ 1.1 & 298.3 & 2.0 & 299.2 & 1.25 & -2.00 \\ \text { OIL TEMPERATURES } & 1.2 & 298.8 & 1.17 & -1.53 \\ & & 23.2 & 29.6 & 25.8\end{array}$

W TH PHI HT POT T

$0.55 \quad-85 . \quad 42 . \quad 17.3 \quad 300.3$

-61. 26.9 .8299 .0

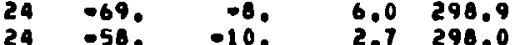

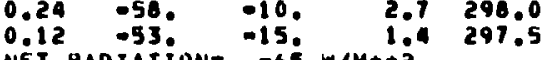
NET RADIATION= $-65 . W / M W E 2$

DAY ITI STARTING TIME
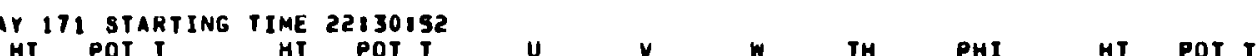

$\begin{array}{rrrrr}1.26 & -3.58 & 0.44 & -71 . & 11 . \\ 2.19 & -3.22 & 0.03 & -56 . & -7 . \\ 2.48 & -2.85 & 0.40 & -49 . & 1 . \\ 2.28 & -1.71 & 0.31 & -37 . & -0 . \\ 1.68 & -0.25 & -0.41 & -8 . & -21 .\end{array}$

$\begin{array}{rrr}0.28 & -1.71 & 0.31 \\ .0 .08 & -0.25 & -0.41\end{array}$

$\checkmark \quad V \quad W \quad T H \quad P H I$

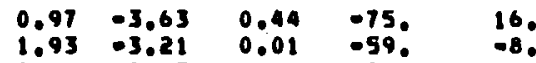
.21 $-2.03 \quad 0.48$ $0.06-1.73 \quad 0.33$ $0.74-0.29-0.46$ $-52$. $-10$.

1.

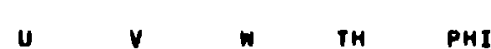

$0.64-3.49 \quad 0.34 \quad-76 . \quad 14$.

$1.85-3.04 \quad 0.03 \quad-99$. $2.16 \quad-2.71 \quad 0.44 \quad-51$. $\begin{array}{rrrr}2.01 & -1.64 & 0.34 & -39 \\ 1.74 & -0.29 & 0.0 .46 & -10 .\end{array}$

०. 1.23.

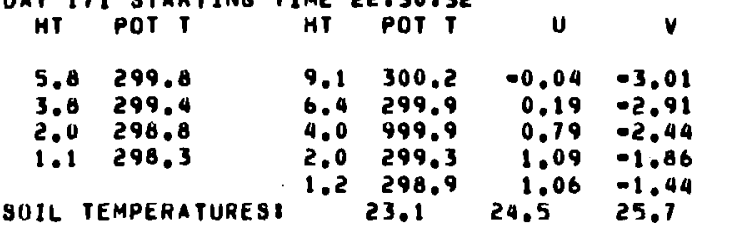

$\begin{array}{lll}W & T H \\ 0.45 & -91 . \\ 0.54 & -86 . \\ 0.25 & -72 . \\ 0.24 & -60 . \\ 0.13 & -53\end{array}$

PI POT

U $V W$ TH PHI

$\begin{array}{lllll}0.25 & -72 . & -4 . & 6.0 & 299.1 \\ 0.24 & -60 . & -8 . & 2.7 & 298.1 \\ 0.13 & -53 & -14 . & 1.4 & 297.5\end{array}$

$\begin{array}{rrrrr}0.53 & -3.07 & 0.37 & -80 . & 26 . \\ 1.64 & -2.78 & 0.04 & -59 . & -7 . \\ 2.03 & -2.62 & 0.50 & -52 . & 6 . \\ 2.05 & -1.59 & 0.33 & -38 . & 11 \\ 1.74 & -0.21 & -0.33 & -7 . & -21 .\end{array}$ $23.1 \quad 24.5 \quad 25.7$ 
JUNE 19-20, 1980; 1841-0251 PST

TONER A

TII) H

TOWER C

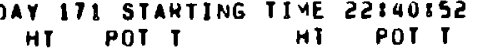

$\begin{array}{rrrrrr}5.8 & 299.8 & 9.1 & 300.1 & -0.53 & -2.67 \\ 3.8 & 299.5 & 6.4 & 299.8 & -0.33 & -2.55 \\ 2.0 & 299.0 & 4.0 & 999.9 & 0.25 & -2.13\end{array}$

1.1298 .5

$2.0 \quad 299.4 \quad 0.66 \quad-1.74$

SOIL TEMPERATURESB

$22.9024 .3 \quad 25.5$

\begin{tabular}{|c|c|}
\hline $\begin{array}{l}0.37 \\
0.44 \\
0.26 \\
0.29 \\
0.19\end{array}$ & $\begin{array}{l}140 \\
-97 . \\
-83 \text {. } \\
-69 \\
-64 .\end{array}$ \\
\hline
\end{tabular}

HI HT PUT

u $v$

$\begin{array}{rr}-0.19 & -2.71 \\ 0.81 & -2.35\end{array}$

$\begin{array}{ll}0.81 & -2.35 \\ 1.20 & -2.20\end{array}$

DAY 171 STARTING TIME 22850152

MET RADIATIONE $-5,5, W / M$. 2

$\begin{array}{lllll}1.29 & -0.32 & -0.37 & -49 . & 7 .\end{array}$

$\begin{array}{ll}5.8 & 299.7 \\ 3.8 & 299.4\end{array}$

$9.1 \quad 299.9 \quad-0.54 \quad-2.68$

TH

PHI HT POT T

$0.40 \quad 123 . \quad 17.3 \quad 299.9$

2.0298 .9

$\begin{array}{llll}6.4 & 299.5 & -0.39 & -2.61\end{array}$

$\begin{array}{llll}2.0 & 999.9 & 0.17 & -2.10 \\ 1.2 & 290.1 & 0.58 & -1.71\end{array}$

SOIL TEMPERATURE S: $\begin{array}{cccc}1.2 & 298.8 & 0.64 & -1.34 \\ 22.8 & 24.2 & 25.3\end{array}$

$0.27-71 . \quad 4.02 .0290 .0$

$0.18 \quad-65 . \quad-5 . \quad 1.4297 .3$ 1.4

DAY ITI STARIING TIME 2310852
HI POIT THT POT

$\begin{array}{ll}5.8 & 299.5 \\ 3.8 & 299.1 \\ 2.0 & 298.6\end{array}$

$\begin{array}{ll}2.0 & 298.6 \\ 1.1 & 298.2\end{array}$

ณ

SOIL TEMPERATURES:

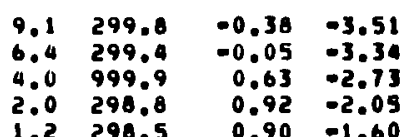

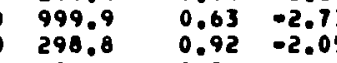

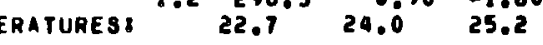

OAY 171 STARTING TIME 23310153

$\begin{array}{ll}5.8 & 299.4 \\ 3.8 & 299.0 \\ 2.0 & 298.4 \\ 1.1 & 298.0\end{array}$

SOIL TEMPERATURES:

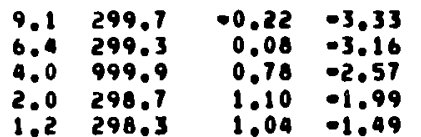

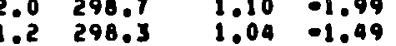

TARTING TIME $23: 20153$

\begin{tabular}{|c|c|c|c|c|}
\hline$w$ & TH & PHI & HT & POT \\
\hline $\begin{array}{l}0.48 \\
0.54 \\
0.26 \\
0.330 \\
0.16 \\
\text { NET RAC }\end{array}$ & $\begin{array}{l}-96 . \\
-91: \\
-77: \\
-66: \\
-61 \text { : }\end{array}$ & $\begin{array}{r}107 . \\
750 \\
10 \\
-30 \\
-11 \\
-60\end{array}$ & $\begin{array}{r}17.3 \\
9.8 \\
6.0 \\
2.7 \\
1.4 \\
1.4\end{array}$ & $\begin{array}{l}299.9 \\
299.1 \\
298.4 \\
297.6 \\
297.0\end{array}$ \\
\hline
\end{tabular}

u v

$0.18-3.04$

$1.25-2.41$

$1.39-0.33 \quad-0.51$

$\begin{array}{ccccr}U & V & W & T H & P H I \\ 0.23 & -3.55 & 0.39 & -86 . & 52 . \\ 1.55 & -3.04 & 0.04 & -63 . & -7 . \\ 1.95 & -2.84 & 0.44 & -55 . & 4 . \\ 1.92 & -1.82 & 0.036 & -44: & 2 . \\ 1.69 & -0.33 & -0.50 & -11 . & -25 .\end{array}$

W TH PHI HT POT

$\begin{array}{lllll}\text { U } & \text { N } & \text { IH } & \text { PHI }\end{array}$

$0.52 \quad-94 . \quad 92 . \quad 17.3299 .9$

0.26 -73.

$0.27-61$.

NET RAOIATION= -66.W/MMLI

$\begin{array}{rrrrr}0.28 & -3.46 & 0.34 & -85 . & 42 . \\ 1.63 & -3.03 & 0.02 & -62 . & -8.0 \\ 2.07 & -2.01 & 0.49 & -54 . & 5 . \\ 2.07 & -1.65 & 0.36 & -39 . & 20 \\ 1.70 & -0.23 & -0.39 & -7 . & -21 .\end{array}$

\begin{tabular}{|c|c|c|c|c|c|c|c|c|c|}
\hline $\boldsymbol{w}$ & TH & PHI & HT & POT $T$ & u & $v$ & $w$ & TH & PHI \\
\hline $\begin{array}{l}53 \\
54 \\
.20 \\
.21 \\
12\end{array}$ & $\begin{array}{l}-86, \\
-82 . \\
-70 \\
-60 \\
-55 .\end{array}$ & $\begin{array}{r}47, \\
30, \\
-9 . \\
-8 . \\
-15,\end{array}$ & $\begin{array}{r}17.3 \\
9.8 \\
6.0 \\
2.7 \\
1.0\end{array}$ & $\begin{array}{l}299.9 \\
299.3 \\
298.3 \\
297.5 \\
297.0\end{array}$ & $\begin{array}{l}0.94 \\
2.08 \\
2.91 \\
2.114 \\
1.79\end{array}$ & $\begin{array}{l}-3.65 \\
-3.34 \\
-2.89 \\
-1.80 \\
-0.31\end{array}$ & $\begin{array}{r}0.39 \\
0.03 \\
0.44 \\
0.35 \\
-0.49\end{array}$ & $\begin{array}{l}-76 . \\
-58 . \\
-50 \\
-40 . \\
-10 .\end{array}$ & $\begin{array}{r}140 \\
-70 \\
20 \\
10 \\
-230\end{array}$ \\
\hline
\end{tabular}

\subsection{9 .3 \\ $\begin{array}{ll}3.8 & 299.0 \\ 2.0 & 298.5\end{array}$ \\ $1.1298,0$}

$\begin{array}{ll}0.22 & -3.42 \\ 0.45 & -3.30\end{array}$ $\begin{array}{ll}0.45 & -3.30 \\ 1.00 & -2.68\end{array}$ $\begin{array}{llll}4.0 & 999.9 & 1.00 & -2.68 \\ 2.0 & 298.7 & 1.21 & -2.11 \\ 1.2 & 298.3 & 1.13 & -1.62\end{array}$ SOIL TEMPERATURES

DAY ITI STARTING TIME 23830153 NET RADIATION= $-67 . W / M A 2$

10 .

23.

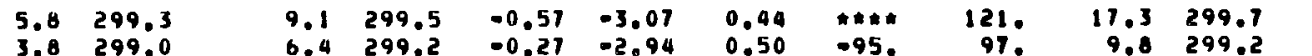

$2.0298 .4 \quad 4.0999 .9 \quad 0.44 \quad-2.37$

$0.82-1.87$

SOIL TEMPERATURESI
$\begin{array}{ccccc}W & T H & P H I & H T & P O T T \\ 0.44 & 1.11 & 121 . & 17.3 & 299.7 \\ 0.50 & -95 . & 97 . & 9.8 & 299.2 \\ 0.25 & -80 . & 9 . & 6.0 & 298.3 \\ 0.30 & -66 . & -1 . & 2.7 & 291.4 \\ 0.17 & -60 . & -9 . & 1.4 & 296.8\end{array}$

NET RADIATION= -66, WIMAME
$\begin{array}{ccccr}U & V & W & T H & \text { PHI } \\ -0.09 & -3.29 & 0.35 & -92 . & 96 . \\ 1.32 & -2.85 & 0.04 & -65 . & -7 . \\ 1.75 & -2.68 & 0.50 & -57 . & 8 . \\ 1.82 & -1.69 & 0.36 & -43 . & 3 . \\ 1.61 & -0.30 & -0.48 & -10 . & -25 .\end{array}$ 
JUNE 19-20, 1980; 1841-0251 PST

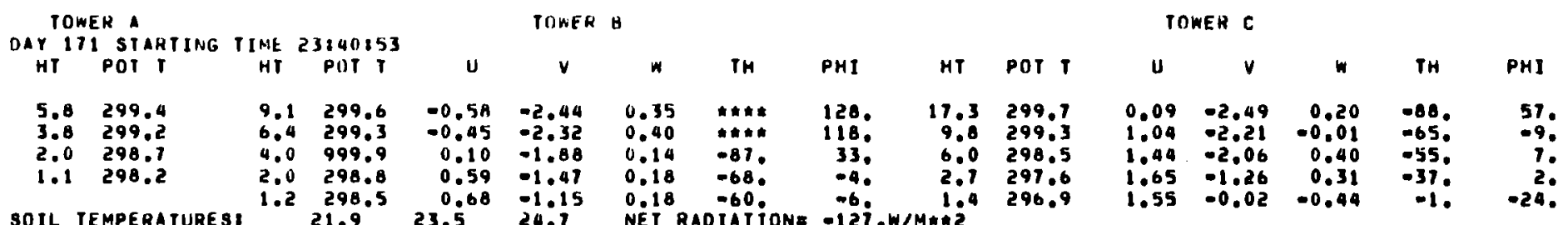

SOIL TEMPERATURESI 21.9 23.5 24.9 NET RAOIATIONE-12i.WMMAE

DAY 171 STARTING TIME 23850853

$\begin{array}{rrrrrr}5.8 & 299.1 & 9.1 & 299.3 & -0.68 & -2.70 \\ 3.8 & 298.9 & 6.4 & 298.9 & -0.48 & -2.71 \\ 2.0 & 298.4 & 4.0 & 999.9 & 0.16 & -2.11 \\ 1.1 & 291.9 & 2.0 & 298.5 & 0.54 & -1.70 \\ & & 1.2 & 298.2 & 0.59 & -1.30\end{array}$

SOIL TEMPERATURES $\begin{array}{lll}290.2 & 0.59 & -1.30 \\ 21.9 & 23.3 & 24.5\end{array}$

$0.17 \quad-3.04 \quad 0.34$

$0.99 \quad-2.58 \quad 0.10$

$1.36-2.45 \quad 0.47$

$\begin{array}{rrr}1.44 & -1.73 & 0.42 \\ 1.34 & -0.40 & -0.60\end{array}$

\section{$\begin{array}{llllll}5.8 & 298.9 & 9.1 & 299.1 & -0.51 & -2.67\end{array}$}

$\begin{array}{llllll}3.8 & 298.7 & 6.4 & 298.8 & -0.40 & -2.59 \\ 2.0 & 298.2 & 4.0 & 999.9 & 0.17 & -2.10\end{array}$

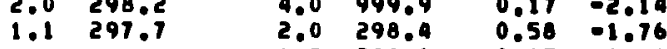

0.37 ..t. 124. 17.3299 .3

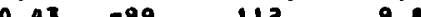

$0.20 \quad-86, \quad 29 . \quad 6.0298 .1$ 0.35 -75. $8.0 \quad 2.7297 .2$ $0.24 \quad-65$-1: 1.4296 .6

$\begin{array}{ccccc}U & V & W & T H & P H I \\ 0.12 & -2.82 & 0.28 & -92 . & 105 . \\ 1.00 & -2.53 & 0.11 & -68 . & -2 . \\ 1.40 & -2.39 & 0.52 & -60 . & 12 . \\ 1.56 & -1.64 & 0.40 & -46 . & 6 . \\ 1.47 & -0.30 & -0.49 & -11 . & -27 .\end{array}$

DAY 172 STARTING TIME OIIOI54

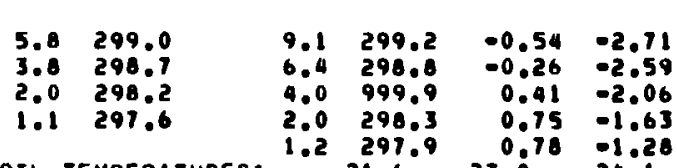
$1.1297 .6 \quad 2.0298 .3 \quad 0.75-1.63$ SOIL TEMPERATURESI $1.221 .6 \quad 23.0 \mathrm{O} 24.1$
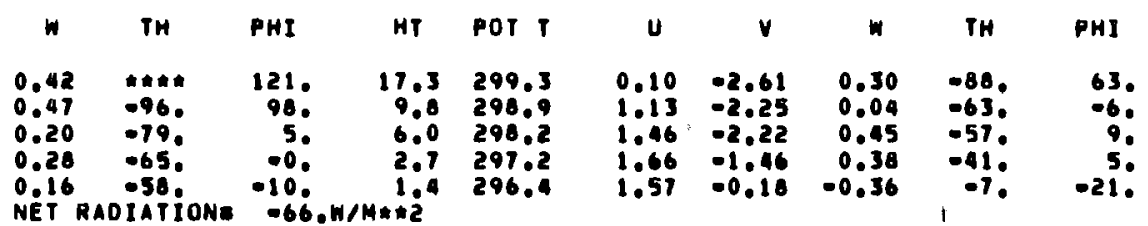

\begin{tabular}{|c|c|c|c|c|c|}
\hline BAY 172 & $\begin{array}{l}\text { START ING } \\
\text { POT T }\end{array}$ & $\begin{array}{r}\text { TIME } \\
\text { HT }\end{array}$ & $\begin{array}{c}0120854 \\
\text { POT T }\end{array}$ & U & $v$ \\
\hline $\begin{array}{l}5.8 \\
3.8 \\
2.0 \\
1.1\end{array}$ & $\begin{array}{l}298.8 \\
298.5 \\
298.0 \\
291.5\end{array}$ & $\begin{array}{l}9.1 \\
6.4 \\
9.0 \\
2.0 \\
1.2\end{array}$ & $\begin{array}{l}299.0 \\
298.7 \\
999.9 \\
298.2 \\
291.0\end{array}$ & $\begin{array}{r}-0.61 \\
-0.32 \\
0.29 \\
0.68 \\
0.72\end{array}$ & $\begin{array}{l}-2.59 \\
-2.52 \\
-2.06 \\
-1.64 \\
-1.25\end{array}$ \\
\hline
\end{tabular}

\begin{tabular}{|c|c|c|c|c|}
\hline$r$ & TH & PHI & HT & POT \\
\hline $\begin{array}{l}0.42 \\
0.47 \\
0.21 \\
0.29 \\
0.20\end{array}$ & $\begin{array}{l}111 \% \\
-97 . \\
-82 \\
-67 \\
-60\end{array}$ & $\begin{array}{r}125 . \\
104, \\
15 . \\
2 . \\
-5 .\end{array}$ & $\begin{array}{r}17.3 \\
9.8 \\
6.0 \\
2.7 \\
1.4\end{array}$ & $\begin{array}{l}299.1 \\
290.7 \\
297.9 \\
297.0 \\
296.3\end{array}$ \\
\hline
\end{tabular}

$\begin{array}{ccccr}U & V & & T H & P H I \\ -0.25 & -2.72 & 0.30 & -95 . & 121 . \\ 1.08 & -2.31 & 0.08 & -66 . & -4 . \\ 1.52 & -2.36 & 0.45 & -57 . & 8 . \\ 1.67 & -1.59 & 0.40 & -44 . & 5 . \\ 1.52 & -0.24 & -0.42 & -9 . & -24 .\end{array}$
NET RAOIATIONA -66 .WTM
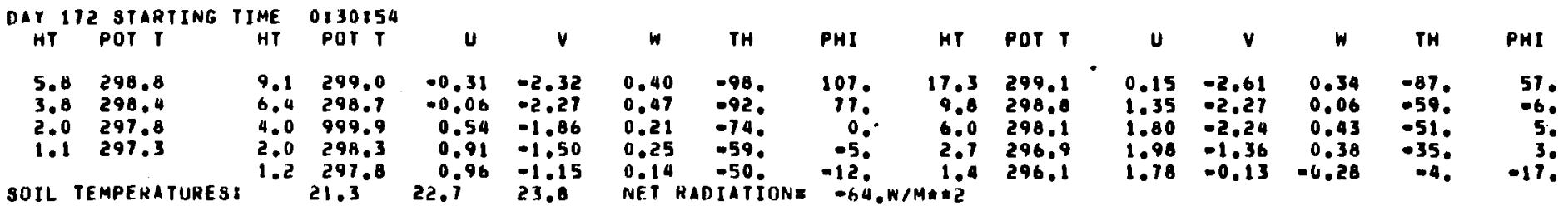
JUNE $19-20,1980 ; 1841-0251$ PST

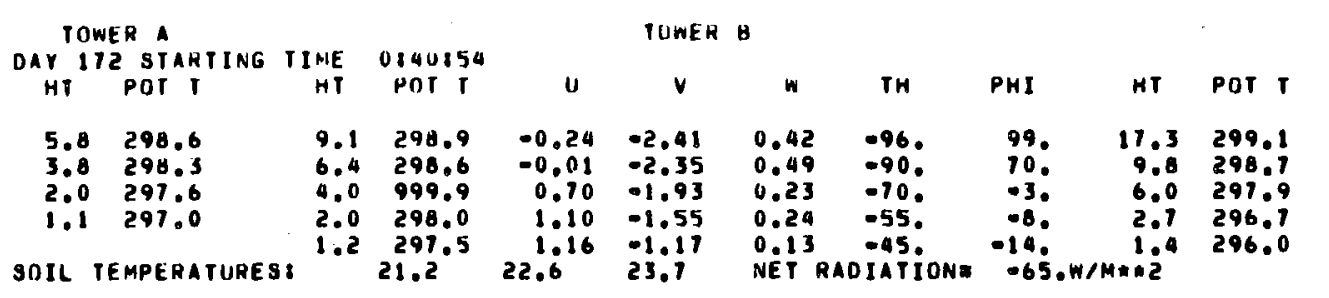

TOWER $C$

DAY 172 STARTING TIME 0850154

\begin{tabular}{|c|c|c|c|c|c|c|c|c|c|c|c|c|c|c|c|}
\hline 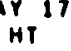 & POT T & HI & POT 1 & u & $v$ & $\omega$ & TH & PHI & HT & POT & u & $v$ & $w$ & TH & PHI \\
\hline $\begin{array}{l}5.8 \\
3.8 \\
2.0 \\
1.1\end{array}$ & $\begin{array}{l}298.5 \\
298.2 \\
297.6 \\
297.1\end{array}$ & $\begin{array}{l}9.1 \\
6.4 \\
4.0 \\
2.0 \\
1.2\end{array}$ & $\begin{array}{l}298.8 \\
298.5 \\
999.9 \\
298.1 \\
297.6\end{array}$ & $\begin{array}{r}-0.42 \\
-0.19 \\
0.40 \\
0.83 \\
0.89\end{array}$ & $\begin{array}{l}-2.39 \\
-2.29 \\
-1.85 \\
-1.52 \\
-1.16\end{array}$ & $\begin{array}{l}0.42 \\
0.47 \\
0.26 \\
0.28 \\
0.17\end{array}$ & $\begin{array}{l}-111 . \\
-95 . \\
-78 . \\
-610 \\
-52 .\end{array}$ & $\begin{array}{r}114 . \\
91 . \\
12 . \\
-20 \\
-10\end{array}$ & $\begin{array}{r}11.3 \\
9.8 \\
6.0 \\
2.7 \\
1.4\end{array}$ & $\begin{array}{l}290.9 \\
290.6 \\
297.9 \\
296.9 \\
296.1\end{array}$ & $\begin{array}{l}0.10 \\
1.23 \\
1.63 \\
1.60 \\
1.67\end{array}$ & $\begin{array}{l}-2.60 \\
-2.23 \\
-2.24 \\
-1.48 \\
-0.19\end{array}$ & $\begin{array}{r}0.30 \\
0.05 \\
0.49 \\
0.40 \\
-0.35\end{array}$ & $\begin{array}{l}-88 . \\
-61 . \\
-54 . \\
-39 . \\
-6 .\end{array}$ & $\begin{array}{r}64 . \\
-6 . \\
7 . \\
40^{\circ} \\
-20 .\end{array}$ \\
\hline
\end{tabular}

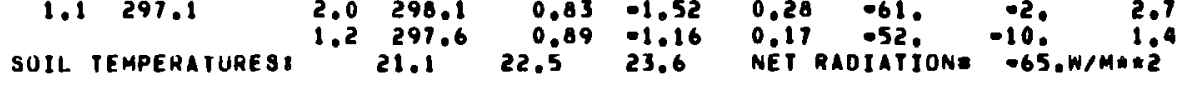

$\begin{array}{lllll}U & V & W & T H & P H I \\ 0.07 & -2.74 & 0.33 & -89 . & 70 . \\ 1.31 & -2.54 & 0.08 & -63 . & -5 . \\ 1.91 & -2.57 & 0.45 & -53 . & 5 . \\ 2.04 & -1.58 & 0.38 & -38 . & 2 . \\ 1.76 & -0.21 & -0.37 & -7 & -20 .\end{array}$

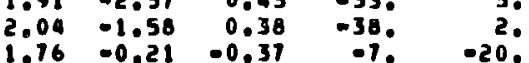

DAY 175 STARTING TIME

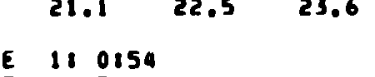

in PHI

$\begin{array}{ccccr}U & V & W & T H & P H I \\ 0.53 & -2.41 & 0.32 & -70 . & 22 . \\ 1.47 & -2.26 & 0.05 & -51 . & -6 . \\ 1.02 & -2.20 & 0.46 & -50 . & 6 . \\ 1.92 & -1.27 & 0.39 & -34 . & 3 . \\ 1.10 & -0.11 & -0.20 & -4 . & -18 .\end{array}$

๘ SOLL TEMPERATUREs:

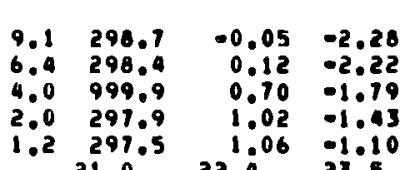

$\begin{array}{ll}0.42 & -91 . \\ 0.46 & -67 . \\ 0.16 & -68 . \\ 0.24 & -55 . \\ 0.14 & -46 .\end{array}$

$\begin{array}{rrr}16 . & 17.3 & 290.8 \\ 55 . & 9.8 & 290.6 \\ -8 . & 6.0 & 297.9 \\ -8 . & 2.7 & 296.7 \\ -13 & 1.4 & 296.0\end{array}$

NET RAOTATIONE - -63. W/MOA

DAY 112 STARTING TIME 1310155

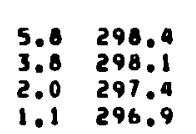

POT $T$ U V

IH PHI

HT POT T

$u$

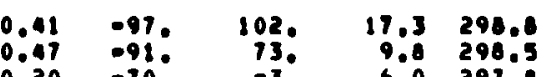

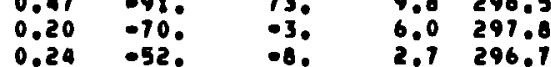

1.1296 .9

$\begin{array}{llll}9.1 & 298.1 & -0.26 & -2.15 \\ 6.4 & 298.4 & -0.03 & -2.11\end{array}$

$4.0 \quad 999.9 \quad 0.63 \quad-1.70$

$\begin{array}{llll}2.0 & 297.9 & 1.03 & -1.33\end{array}$

SOIL TEMPERATURESI

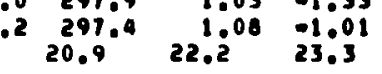

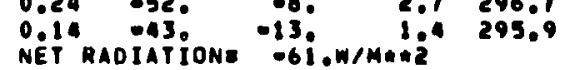

$\begin{array}{rrrrr}0.22 & -2.30 & 0.28 & -85 . & 44 . \\ 1.29 & -2.12 & 0.09 & -59 . & -4 . \\ 1.71 & -2.14 & 0.49 & -51 . & 7 . \\ 1.93 & -1.28 & 0.43 & -34 . & 4 . \\ 1.11 & -0.11 & -0.20 & -4 . & -18 .\end{array}$

DAY 172 STARTING TIME 1220855

$\begin{array}{llllll}\text { HI POI T } & \text { HT } & \text { POT T } & U & V \\ 5.8 & 298.3 & 9.1 & 298.7 & -0.08 & -2.31 \\ 3.8 & 298.0 & 6.4 & 298.3 & 0.16 & -2.31 \\ 2.0 & 297.4 & 4.0 & 999.9 & 0.78 & -1.89 \\ 1.1 & 296.9 & 2.0 & 297.8 & 1.14 & -1.50 \\ & 1.2 & 297.4 & 1.16 & -1.13 \\ \text { OIL TEMPERATURES } & & 20.8 & 22.1 & 23.2\end{array}$

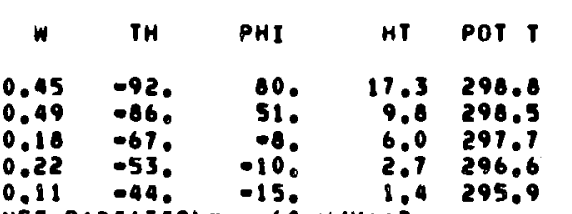

$\begin{array}{ccccr}U & V & W & T H & P H I \\ 0.35 & -2.54 & 0.34 & -82 . & 36 . \\ 1.30 & -2.35 & 0.05 & -60 . & -6 . \\ 1.83 & -2.32 & 0.46 & -52 . & 6 . \\ 1.95 & -1.37 & 0.39 & -35 . & 3 . \\ 1.69 & -0.16 & -0.32 & -5 . & -19 .\end{array}$

SOIL TEMPERATURES

$20.822 .1^{16} 23.2$

NET RADIATIONE - -60 . WIMALE

DAY 172 SIARTING TIME 1130855

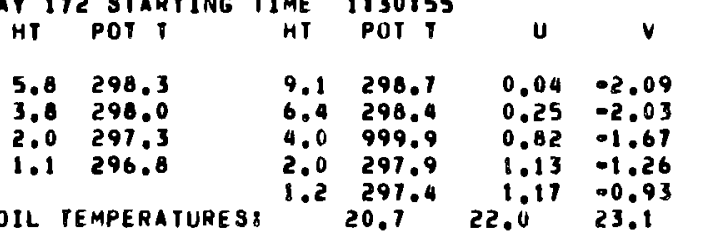

$\begin{array}{rrrrr}W & T H & \text { PHI } & \text { HT } & \text { POT T } \\ 0.45 & -89 . & 64 . & 17.3 & 298.8 \\ 0.50 & -83 . & 43 . & 9.8 & 298.5 \\ 0.21 & -64 . & -7 . & 6.0 & 297.9 \\ 0.24 & -48 . & -9 . & 2.7 & 296.7 \\ 0.15 & -39 & -13 . & 1.4 & 296.0\end{array}$

$\begin{array}{lllll}U & V & \text { TH } & \text { PHI }\end{array}$

$\begin{array}{lllll}0.55 & -2.12 & 0.29 & -16 . & 20 .\end{array}$

$\begin{array}{lllll}1.48 & -2.06 & 0.06 & -54 . & -6 . \\ 1.90 & -1.99 & 0.45 & -46 . & 5\end{array}$

$\begin{array}{rrrrr}1.90 & -1.99 & 0.45 & -46 . & 5 . \\ 2.03 & -1.12 & 0.37 & -29 . & 2 . \\ 1.78 & -0.05 & -0.21 & -2 . & -15 .\end{array}$

SOIL TEMPERATURES:

NET RADIATIONE -59 . WIMATL 
JUNE $19-20,1980 ; 1841-0251$ PST

\begin{tabular}{|c|c|c|c|c|c|c|c|c|c|c|}
\hline 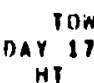 & $\begin{array}{l}\text { ER A } \\
\text { Z SIAKIING } \\
\text { POT T }\end{array}$ & IIME & $\begin{array}{c}1840855 \\
\text { Por }\end{array}$ & u & TUWER & W & TH & PHI & HT & POT \\
\hline $\begin{array}{l}5.8 \\
3.8 \\
2.0 \\
1.1\end{array}$ & $\begin{array}{l}298.3 \\
298.0 \\
297.5 \\
297.0\end{array}$ & $\begin{array}{l}9.1 \\
6.4 \\
4.0 \\
2.0 \\
1.2\end{array}$ & $\begin{array}{l}298.7 \\
298.4 \\
999.9 \\
298.0 \\
297.5\end{array}$ & $\begin{array}{r}-0.06 \\
0.13 \\
0.68 \\
1.08 \\
1.15\end{array}$ & $\begin{array}{l}-1.71 \\
-1.72 \\
-1.46 \\
-1.12 \\
-0.83\end{array}$ & $\begin{array}{l}0.42 \\
0.43 \\
0.19 \\
0.23 \\
0.15\end{array}$ & $\begin{array}{l}-92 \\
-86 \\
-65 \\
-46 \\
-36\end{array}$ & $\begin{array}{r}18 . \\
52 . \\
-5 . \\
-9 . \\
-13 .\end{array}$ & $\begin{array}{r}17.3 \\
9.8 \\
6.0 \\
2.7 \\
1.4\end{array}$ & $\begin{array}{l}298.0 \\
298.6 \\
298.0 \\
296.9 \\
295.9\end{array}$ \\
\hline
\end{tabular}

1850855

HT 172 STIARTING TIME 1850855

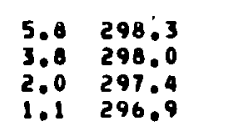

SOIL TEMPERATURES:

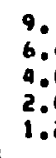

$9.1 \quad 298.7$ $\begin{array}{llll}6.4 & 298.04 & 0.14 & -1.94 \\ 0.32 & -1.92\end{array}$ $\begin{array}{llll}9.0 & 999.9 & 0.89 & -1.60 \\ 2.0 & 297.9 & 1.29 & -1.17\end{array}$ $\begin{array}{llll}2 & 297.9 & 1.29 & -1.17 \\ & 297.3 & 1.36 & -0.83\end{array}$

SOIL TEMPERATURES: $20.6^{2} 21.9^{2} 2.9$

DAY 172 STARTING TIME 210856

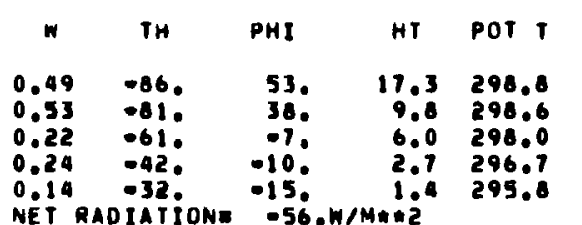

NET RADIATIONE -156 . \begin{tabular}{lccrr}
\multicolumn{5}{c}{ TOWER C } \\
$U$ & $V$ & $W$ & $T H$ & PHI \\
$U$ & $V$ & & & \\
.57 & -1.89 & 0.29 & -13. & 19. \\
40 & -1.81 & 0.07 & -52. & -6. \\
15 & -1.77 & 0.47 & -45. & 7. \\
97 & -1.04 & 0.45 & -28. & 5. \\
.17 & -0.01 & -0.19 & -0. & -14.
\end{tabular}

$1.17 \quad-0.01 \quad-0.19 \quad-0 . \quad-14$.

\subsection{8 .3 \\ 2.0297 .3}

๘

SOIL TEMPERATURES: DAT 172 STAHTING TIMET
HT

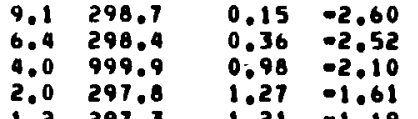

$2.0297 .8 \quad 1.27 \quad-1.61$

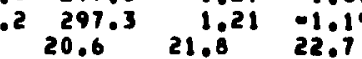

HT POT T

$\begin{array}{lllll}0.51 & -87 . & 53 . & 17.3 & 298.8 \\ 0.55 & -82 . & 36 . & 9.8 & 298.5\end{array}$

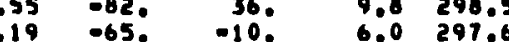

$0.23 \quad-52, \quad-11$. 2.7296 .6

NET RAOIATIONE -56.WMMES 296.0

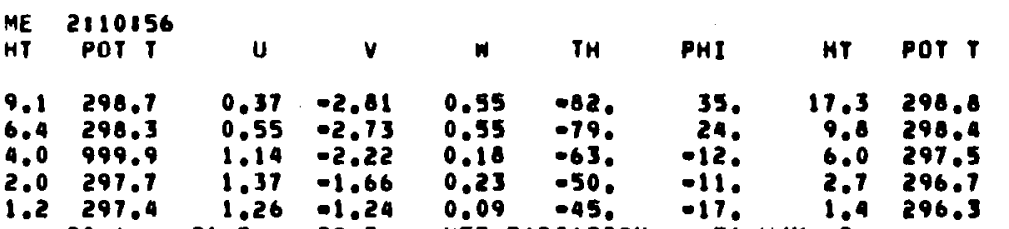

SOIL TEMPERATUREg:

$.2297 .4 \quad 1.26 \quad-1.24$

NET RAOIATIONE - -56 . W/MALE

DAY 172 STARTING TIME 2320156

\subsection{8 .3 \\ 2.0297 .4 \\ 1.1297 .0}

SOIL TEMPERATURES

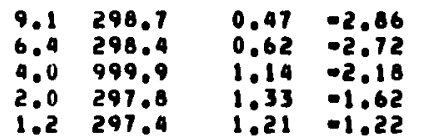

$\begin{array}{rrrr}2.0 & 297.8 & 1.33 & -1.62 \\ 1.2 & 297.4 & 1 . .21 & -1.22 \\ & 20.5 & 21.6 & 22.5\end{array}$

W TH PHI

HT POT T

$\begin{array}{ll}0.59 & -81 . \\ 0.56 & -77 .\end{array}$

$0.17 \quad-62$.

$0.21 \quad-51$ : -12. NET RAOIATIONE - -55 .W/MUIRE

DAY 172 STARTING TIME

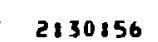

5.8 298.3

9.

2.0297 .4

$\begin{array}{llll}9.1 & 298.8 & 0.63 & -3.18\end{array}$

$\begin{array}{llll}4.0 & 949.9 & 1.30 & -3.043\end{array}$

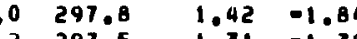

SOIL TEMPERATURES:

297.5

$\begin{array}{ll}1.42 & -1.84 \\ 1.31 & -1.39\end{array}$

W TH PHI HT POT

$0.59-79$.

$\begin{array}{ll}0.52 & -75 . \\ 0.17 & -62 .\end{array}$

22. $17.3 \quad 298.9$

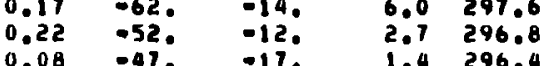

$\begin{array}{rrr}12 . & 9.6 & 298.4 \\ -14 . & 6.0 & 297.6\end{array}$

NET RADIATION= - -56.

$\begin{array}{ccccr}U & V & W & T H & P H I \\ 0.80 & -2.16 & 0.37 & -70 . & 17 . \\ 1.60 & -2.06 & 0.09 & -52 . & -5 . \\ 1.91 & -2.01 & 0.52 & -46 . & 7 . \\ 2.05 & -1.14 & 0.43 & -29 . & 4 . \\ 1.76 & -0.06 & -0.22 & -2 . & -15 .\end{array}$

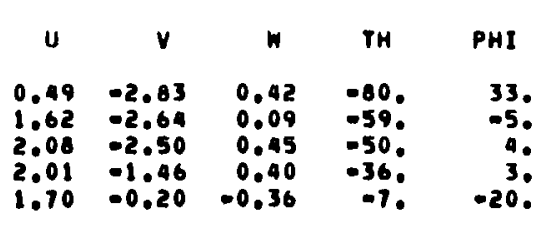

$1.70=0.20 \quad-0.36$

$\begin{array}{ccccr}U & V & W & T H & \text { PHI } \\ 0.61 . & -3.07 & 0.42 & -79 . & 26 . \\ 1.78 & -2.03 & 0.03 & -58 . & -7 . \\ 2.21 & -2.57 & 0.41 & -49 . & 2 . \\ 2.07 & -1.52 & 0.36 & -36 . & 2 . \\ 1.72 & -0.22 & -0.38 & -7 . & -21 .\end{array}$

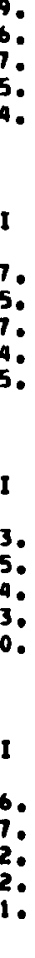

$\begin{array}{ccccc}U & V & W & T H & P H I \\ 0.96 & -3.00 & 0.39 & -72 . & 14 . \\ 1.95 & -2.79 & -0.01 & -95: & -9.0 \\ 2.31 & -2.52 & 0.37 & -47: & 1: \\ 2.14 & -1.46 & 0.35 & -34: & 1: \\ 1.70 & -0.19 & -0.335 & -6 . & -19:\end{array}$

$\begin{array}{lllll}4 & V & \text { TH } & \text { PHI }\end{array}$

$\begin{array}{lllll}1.06 & -3.38 & 0.43 \quad-73 . & 14 .\end{array}$

$\begin{array}{ccccc}2.10 & -3.09 & 0.02 & -56 . & -8 . \\ 2.40 & -2.70 & 0.41 & -48 . & 1: \\ 2.14 & -1.59 & 0.35 & -37 & 11\end{array}$

$\begin{array}{lllll}2.14 & -1.59 & 0.35 & -37: & 1 \\ 1.78 & -0.24 & -0.41 & -8 . & -21\end{array}$ 
JUNE 19-20, 1980; 1841-0251 PST

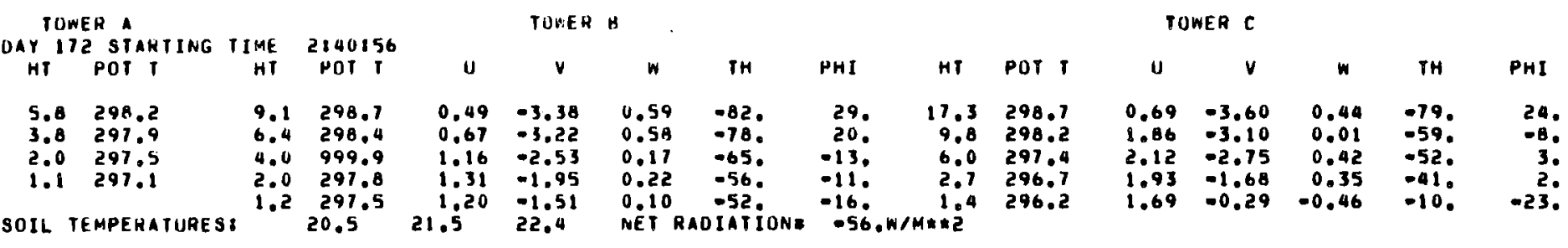


JULY 1-2, 1980; 1920-0200 PST TOWEN A OAY IB3 STARTING TIME 19820121
HT POT I HOT POT

$\begin{array}{lllll}\text { HT POT T } & \text { HT } & \text { POT T } & U \\ 5.8 & 302.4 & 9.1 & 303.1 & 0.92 \\ 3.8 & 302.0 & 6.4 & 302.8 & 1.19 \\ 2.0 & 301.4 & 4.0 & 302.5 & 1.64 \\ 1.1 & 300.7 & 2.0 & 302.0 & 1.93 \\ & & 1.2 & 301.3 & 1.90\end{array}$

TORER H

$\begin{array}{ll}v \\ 2.64 \\ 2.39 & 0.1 \\ 2.27 & 0.1 \\ 1.94 & 0.1 \\ 1.45 & 0.1\end{array}$

OAY 103 STARTING TIME 19830821

$\begin{array}{lll}5.8 & 302.6 & 9 \\ 3.8 & 302.3 & 6 \\ 2.0 & 301.5 & 4 \\ 1.1 & 300.7 & 2\end{array}$

2.0301 .5

9.1303 .2

$\begin{array}{rr}6.4 & 302.8 \\ 4.0 & 302.5\end{array}$

2.0302 .0

SOIL TEMPERATURES?

$1.2 \quad 301.3$

DAY 183 STARTING TIME 19140121

$\begin{array}{lllll}5.8 & 302.7 & 9.1 & 303.2 & 1.19\end{array}$

$\begin{array}{lllll}3.8 & 302.4 & 6.4 & 302.8 & 1.46 \\ 2.0 & 301.3 & 4.0 & 302.5 & 1.92 \\ 1.1 & 300.2 & 2.0 & 301.9 & 2.20\end{array}$

$\omega$ SOLL TEMPERATURES?

DAY 183 SIARTING TIME 1985082

$\begin{array}{llllll}5.8 & 302.8 & 9.1 & 303.2 & 1.18 & 2.26 \\ 3.8 & 302.4 & 6.4 & 302.8 & 1.43 & 2.11 \\ 2.0 & 301.3 & 4.0 & 302.5 & 1.96 & 2.00 \\ 1.1 & 300.1 & 2.0 & 301.8 & 2.27 & 1.68 \\ & & 1.2 & 300.9 & 2.12 & 1.24\end{array}$

SOIL TEMPERATURES:

DAY 183 STARTING TIME 2020121

$\begin{array}{llllll}\text { HT POT T } & \text { HT } & \text { POT } T & U & V \\ 5.6 & 303.0 & 9.1 & 303.5 & 1.20 & 2.30 \\ 3.8 & 302.7 & 6.4 & 303.1 & 1.52 & 2.14 \\ 2.0 & 301.4 & 4.0 & 302.5 & 2.20 & 2.02 \\ 1.1 & 299.9 & 2.0 & 301.5 & 2.50 & 1.62 \\ & 1.2 & 300.5 & 2.30 & 1.14 \\ \text { SOIL TEMPERATURES } & & 27.6 & 29.4 & 29.9\end{array}$

OAY 183 STARTING TIME

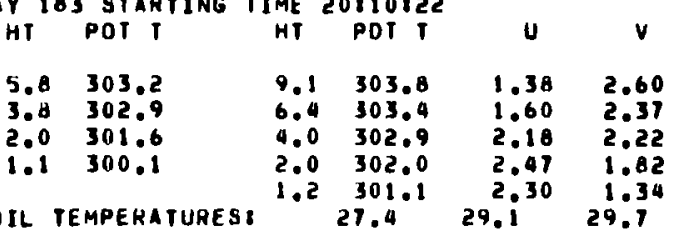

0.15
0.26
0.16
0.19
0.11

$\begin{array}{lrrr}\text { IH } & \text { PHI } & H T & \text { POT T } \\ \text { I1. } & -12 . & 17.3 & 30.3 .1 \\ 64 . & -9 . & 9.8 & 302.9 \\ 54 . & -15 . & 6.0 & 302.3 \\ 45 . & -15 . & 2.7 & 300.6 \\ 37 . & -18 . & 1.4 & 299.6\end{array}$

TOWER C

$\begin{array}{ccccr}U & V & W & T H & \text { PHI } \\ 0.97 & 2.11 & 0.20 & 65 . & 4 . \\ 1.72 & 2.12 & 0.30 & 51 . & 2 . \\ 2.15 & 1.82 & 0.55 & 40 . & 6 . \\ 2.57 & 1.28 & 0.12 & 26 . & -6 . \\ 2.07 & 0.80 & 0.75 & 21 . & 12 .\end{array}$

NET RADIATION= -19. W/MHAZ

$\begin{array}{ccccc}U & V & W & \text { TH } & \text { PHI } \\ 1.00 & 2.24 & 0.22 & 66 . & 4 . \\ 1.10 & 2.22 & 0.35 & 51 . & 3 . \\ 2.21 & 1.91 & 0.55 & 11 . & 6 . \\ 2.40 & 1.31 & 0.12 & 28 . & -6 . \\ 1.90 & 0.80 & 0.76 & 22 . & 13 .\end{array}$

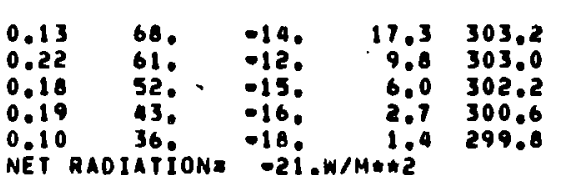

W TH PHI HT POT

$\begin{array}{ccccr}U & V & W & T H & \text { PHI } \\ 1.13 & 2.00 & 0.22 & 61 . & 3 . \\ 1.08 & 2.06 & 0.34 & 48 . & 2 . \\ 2.32 & 1.81 & 0.52 & 38 . & 4 . \\ 2.67 & 1.23 & 0.12 & 25 . & -6 . \\ 2.09 & 0.80 & 0.75 & 21 . & 11 .\end{array}$

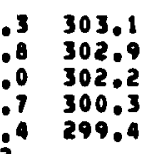

2.09

\begin{tabular}{|c|c|c|c|c|c|c|c|c|c|}
\hline$w$ & IH & PHI & HT & pot & u & $v$ & $w$ & $T H$ & PHI \\
\hline $\begin{array}{l}0.14 \\
0.26 \\
0.23 \\
0.24 \\
0.12\end{array}$ & $\begin{array}{l}62 . \\
56 . \\
46 . \\
37 . \\
30 .\end{array}$ & $\begin{array}{l}-14 . \\
-11 . \\
-14 . \\
-15 . \\
-18 .\end{array}$ & $\begin{array}{r}17.3 \\
9.8 \\
6.0 \\
2.7 \\
1.9\end{array}$ & $\begin{array}{l}303.2 \\
303.0 \\
302.3 \\
300.1 \\
299.1\end{array}$ & $\begin{array}{l}1.17 \\
1.08 \\
2.40 \\
2.81 \\
2.17\end{array}$ & $\begin{array}{l}1.77 \\
1.86 \\
1.69 \\
1.15 \\
0.77\end{array}$ & $\begin{array}{l}0.16 \\
0.29 \\
0.52 \\
0.12 \\
0.72\end{array}$ & $\begin{array}{l}56 . \\
45, \\
35 . \\
22, \\
20 .\end{array}$ & $\begin{array}{r}-0 \\
0 . \\
40 \\
-6 . \\
10 .\end{array}$ \\
\hline
\end{tabular}

MET PADIATIONE -18.2 .

W TH PHI HT PDI

$\begin{array}{ccccr}U & V & W & T H & \text { PHI } \\ 1.33 & 1.60 & 0.24 & 50 . & 2 . \\ 2.02 & 1.71 & 0.30 & 90 . & 0 . \\ 2.42 & 1.53 & 0.55 & 32 . & 5 . \\ 2.97 & 1.10 & 0.13 & 20 . & -6 . \\ 2.25 & 0.71 & 0.71 & 19 . & 9 .\end{array}$

0.13 63. -14. $17.3 \quad 303.3$

0.26 55. -11. 9.8303 .1

0.25 42. $\quad-14.06 .0302 .4$

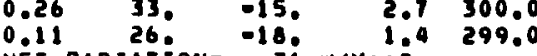

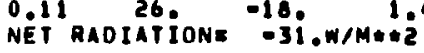

$2.25 \quad 0.170 .7$

$\begin{array}{lllll} & T H & \text { PHI } & \text { HT } & \text { POT T } \\ 0.11 & 62 . & -16 . & 17.3 & 303.5 \\ 0.22 & 56 . & -13 . & 9.8 & 303.2 \\ 0.23 & 46 . & -15 . & 6.0 & 302.5 \\ 0.22 & 36 . & -16 . & 2.7 & 300.1 \\ 0.09 & 30 & -19 . & 1.4 & 299.1\end{array}$

$u$
1.05
1.95
2.94
2.94
2.25

$\begin{array}{cc}v & \\ 1.78 & 0.31 \\ 1.94 & 0.29 \\ 1.67 & 0.48 \\ 1.15 & 0.13 \\ 0.78 & 0.13\end{array}$

$\begin{array}{ll}\text { IH } & \text { PHI } \\ \text { 59: } & 8: \\ 45: & 0: \\ 34: & 3: \\ 21: & -66 \\ 19: & 10 .\end{array}$ 
JULY 1-2, 1980; 1920-0200 PST

TOWER A HT POT T IOJ STARIING TIME 20120152
HT POT T

$\begin{array}{llll}5.8 & 303.1 & 9.1 & 303.6 \\ 3.8 & 302.7 & 6.4 & 303.4 \\ 2.0 & 301.5 & 4.0 & 303.0 \\ 1.1 & 300.1 & 2.0 & 302.3 \\ & & 1.2 & 301.4\end{array}$

$\begin{array}{cc} & \text { TOWEH } \\ U & V \\ 1.36 & 2.82 \\ 1.55 & 2.56 \\ 2.09 & 2.42 \\ 2.50 & 2.05 \\ 2.39 & 1.46 \\ 28.8 & 29.5\end{array}$

TOWE H

TOWER C

SOIL TEMPERATURES:

DAY 183 STARTING TIME 20130122

$\begin{array}{llllll}5.8 & 303.0 & 9.1 & 303.3 & 0.84 & 2.56 \\ 3.8 & 302.6 & 6.4 & 303.0 & 1.14 & 2.32 \\ 2.0 & 301.4 & 4.0 & 302.7 & 1.71 & 2.21 \\ 1.1 & 300.2 & 2.0 & 302.1 & 2.07 & 1.09 \\ & & 1.2 & 301.3 & 2.02 & 1.36\end{array}$

SOIL TEMPERATURES: $\begin{array}{lll}301.3 & 2.02 & 1.36 \\ 26.6 & 26.5 & 29.3\end{array}$

DAY 183 STARTING TIME 20140122
HT POT T HT POT T

$\begin{array}{ll}5.8 & 302.7 \\ 3.8 & 302.3\end{array}$

2.0301 .3

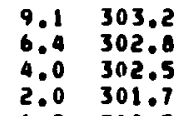

$\begin{array}{ll}4.0 & 302.5 \\ 2.0 & 301.7\end{array}$

$\begin{array}{lll}2.0 & 301.7\end{array}$

SOIL TEMPERATURES\&

$\begin{array}{rl}1.90 & 1.97 \\ 2.33 & 1.66\end{array}$

DAY 183 STARTING TIME 20850122

$\begin{array}{llllll}5.8 & 303.0 & 9.1 & 303.6 & 1.30 & 1.97 \\ 3.8 & 302.8 & 6.4 & 303.3 & 1.56 & 1.80 \\ 2.0 & 301.0 & 4.0 & 302.9 & 2.20 & 1.80 \\ 1.1 & 299.8 & 2.0 & 301.1 & 2.78 & 1.55 \\ & 1.2 & 300.3 & 2.61 & 1.00 \\ & & 1.00 \\ & & 26.0 & 21.9 & 28.8\end{array}$

SOIL TEMPERATURES $26.0 \quad 27.928 .9$

DAY 103 STARTING TIME 2180132

$$
\begin{array}{ll}
5.8 & 303.2 \\
3.8 & 302.9 \\
2.0 & 301.4 \\
1.1 & 300.0
\end{array}
$$

9.1303 .8

6.4
4.0
402.9

$2,0 \quad 302,0$

SOIL TEMPERATURES:

.2301 .1
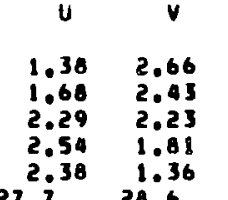

DAY 183 STARTING TIME 21810823
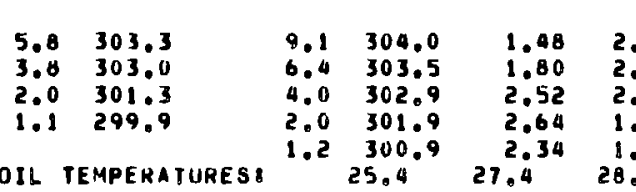

$\begin{array}{lllll}* & T H & \text { PHI } & H T & \text { POT T } \\ 0.08 & 64 . & -17 . & 17.3 & 303.4 \\ 0.18 & 59 . & -14 . & 9.8 & 303.2 \\ 0.19 & 49 . & -16, & 6.0 & 302.3 \\ 0.24 & 39 . & -15, & 2.7 & 300.2 \\ 0.10 & 32 . & -19 . & 1.4 & 299.0\end{array}$

$\begin{array}{ccccr}U & V & W & T H & P H I \\ 0.63 & 2.21 & 0.24 & 79 . & 13 . \\ 1.02 & 2.37 & 0.37 & 59 . & 6 . \\ 2.12 & 1.99 & 0.58 & 43 . & 1 . \\ 2.62 & 1.31 & 0.12 & 27 . & -6 . \\ 2.07 & 0.81 & 0.77 & 21 . & 12 .\end{array}$

NET RADIATIONE -32.W/MME2

$\begin{array}{lllll}2.62 & 1.31 & 0.12 & 27 . & -6 . \\ 2.07 & 0.81 & 0.77 & 21 . & 12 .\end{array}$

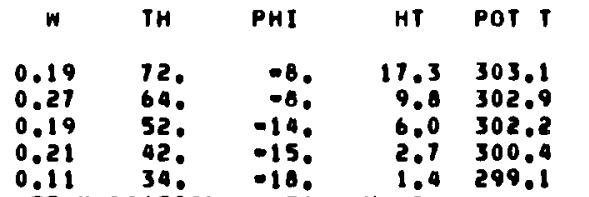

$\begin{array}{ccccc}U & V & W & T H & P H I \\ 0.46 & 2.03 & 0.22 & 11 . & 18 . \\ 1.25 & 2.17 & 0.32 & 60 . & 6 . \\ 1.18 & 1.91 & 0.58 & 97 . & 10 . \\ 2.49 & 1.38 & 0.15 & 30 . & -5 . \\ 2.05 & 0.81 & 0.71 & 22 . & 12 .\end{array}$ NET HADIATION= -3i.W/MAE?

W TH PHI HT POT T

$\begin{array}{ccccc}U & V & W & T H & P H I \\ 1.11 & 1.64 & 0.24 & 56 . & 4 . \\ 1.80 & 1.71 & 0.29 & 45 . & 1 . \\ 2.20 & 1.61 & 0.51 & 35 . & 4 . \\ 2.90 & 1.20 & 0.13 & 22 . & -6 . \\ 2.27 & 0.70 & 0.73 & 19 . & 10 .\end{array}$

0.25 57: $\quad-10 . \quad 9.8302 .9$

0.25 35: - $\quad-15 . \quad 6.0302 .2$

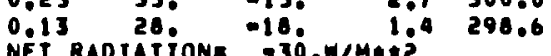

W TH PHI HT POT T

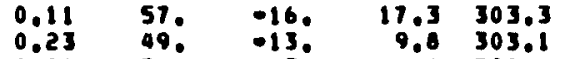

0.24 39. -15. 6.0302 .4

$0.32 \quad 29 . \quad-14.0 \quad 2.7299 .4$ NET RADIATIONE - -30.WIMAME

SOIL TEMPERATURES $\begin{array}{llll}1.2 & 300.9 & 2.34 & 1.18 \\ & 25.4 & 27.4 & 28.4\end{array}$

2.19
1.70

W TH PHI HT POT T

$\begin{array}{lll}1.57 & 1.32 & 0.21 \\ 2.07 & 1.47 & 0.29\end{array}$

$2.56 \quad 1.33 \quad 0.50$

$\begin{array}{lll}3.06 & 0.87 & 0.13 \\ 2.26 & 0.71 & 0.60\end{array}$

(2)

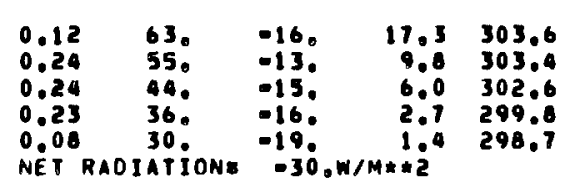

$\begin{array}{ccccr}U & V & W & T H & \text { PHI } \\ 1.32 & 1.77 & 0.330 & 53 . & 5 . \\ 2.04 & 1.93 & 0.37 & 93 . & 2 . \\ 2.59 & 1.71 & 0.50 & 33 . & 3 . \\ 3.02 & 1.10 & 0.13 & 20 . & -6 . \\ 2.31 & 0.16 & 0.71 & 10 . & 9 .\end{array}$

40.
350
20.
16.

$0.11 \quad 61 . \quad-16 . \quad 11.3 \quad 303.8$

$0.27 \quad 54, \quad-12 . \quad 9.35303 .8$

$\begin{array}{lllll}0.28 & 41 . & -14 . & 6.0 & 302.4 \\ 0.26 & 33 . & -15 . & 2.7 & 300.0\end{array}$

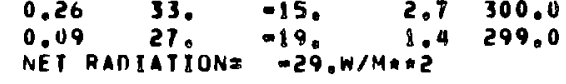

$\begin{array}{lllll}1.17 & 2.24 & 0.35 & 63 . & B . \\ 2.19 & 2.16 & 0.33 & 45 . & 1 . \\ 2.79 & 1.78 & 0.40 & 33 . & -0.0 \\ 3.09 & 1.13 & 0.12 & 20 . & -6.0 \\ 2.36 & 0.19 & 0.76 & 19 . & 10 .\end{array}$ 
JULY 1-2. 1980: 1920-0200 PST

$$
\text { TOWER }
$$

TORER H

TOWER $\mathrm{C}$

DAY 183 STARTING TIME 51850853

$$
\begin{array}{ll}
5.8 & 303.3 \\
3.8 & 303.1 \\
2.0 & 301.3
\end{array}
$$$$
1.1300 .0
$$

v

w TH

SOIL TEMPERATURES

$\begin{array}{ll}9.1 & 304.1 \\ 6.4 & 303.6\end{array}$

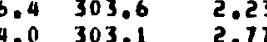

$\begin{array}{lll}0.71 & 2.22\end{array}$

1.68

DAY 183 STARTING TIME 21830123

hi

$\begin{array}{ll}5.8 & 303.3 \\ 3.8 & 303.0 \\ 2.0 & 301.0 \\ 1.1 & 299.8\end{array}$

$\begin{array}{ll}9.1 & 304.0 \\ 6.4 & 303.8 \\ 4.0 & 302.6 \\ 2.0 & 300.7 \\ 1.2 & 299.9 \\ & 25.0\end{array}$

28.2

48. -19. 19.3

0.31 39. $-15 . \quad 6.0302 .3$

$0.29 \quad 30 . \quad-15 . \quad 2.7300 .0$

NET RADIATIONE $-29 . W / M M E$

$\checkmark$

SOIL TEMPERATURES:

2.48
2.47
3.03
3.26
2.80
2.00
.

2.610 .2

H TH PHI

HT POT $T$

$\begin{array}{lllll}0.30 & 46 . & -16 . & 17.3 & 304.0 \\ 2.06 & -14 . & 9.8 & 303.0\end{array}$

$1.44 \quad 0.31$

-140
4.150

28.0

NET RADIATIONE

$\quad 2.7299 .9$

DAY 183 STARTING TIME 21140123

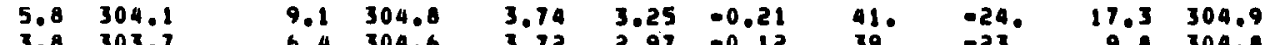

$3.8303 .7 \quad 6.4304 .6 \quad 3.72 \quad 2.97 \quad 0.12 \quad 39.2-23$.

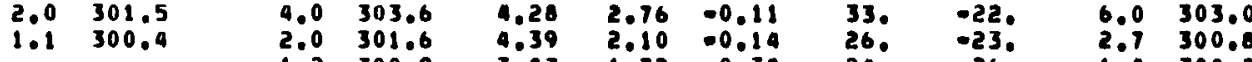

1.1300 .4

SOIL TEMPERATURES:

2.0301 .6

300.923 .03 $28.72-0.38$ NET RAOIATIONE -36. W/MAIIE

DAY 183 STARTING TIME 21850823

Hi bot 1 IME $21850: 23$
HT POT

$\begin{array}{ll}5.8 & 303.1 \\ 3.8 & 302.1\end{array}$

2.0300 .6

$9.1303 .8 \quad 2.60$

v

H PHI HT POT T

$2.11 \quad 2.87$

$3.42, \quad 1.96$

$\begin{array}{ll}3.16 & 1.16 \\ 2.39 & 0.81\end{array}$

0.39
0.37

0.35

0.14

TH PHI

1EMPERATUREB

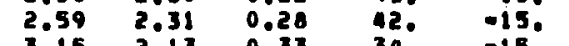

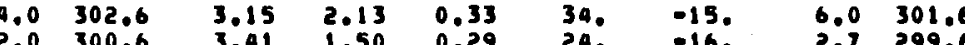

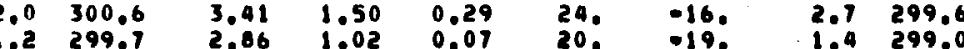

L TEMPERATURES: 29.6

26.6 NET RAOIATIONE - -30.WMMARE

$\begin{array}{ccccc}U & V & W & \text { TH } & \text { PHI } \\ 2.81 & 2.84 & 0.14 & 45 . & -5 . \\ 3.27 & 2.58 & 0.33 & 38 . & -3.3 . \\ 3.73 & 1.97 & 0.33 & 28 . & -3.3 \\ 3.30 & 1.13 & 0.13 & 19 . & -6 . \\ 2.51 & 0.85 & 0.84 & 19 . & 10 .\end{array}$

DAY 183 STARTING TIME 2280123

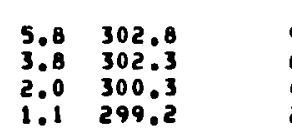

por $T$ U

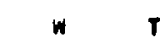

PHI

HT POT 1

$\begin{array}{lll}3.02 & 3.68 & -0.54\end{array}$

$\begin{array}{lll}4.07 & 3.33 & -0.30\end{array}$

$\begin{array}{lll}4.57 & 2.72 & -0.34 \\ 4.04 & 1.99 & -0.53\end{array}$

$\begin{array}{rrr}4.04 & 1.99 & -0.53 \\ 3.26 & 1.66 & 0.10\end{array}$

IH PHI

44. -16

39. -12.

$26 .-16$.

2.0300 .3

9.1303 .5

$\begin{array}{ll}9.9 & 303.3 \quad 2.99 \\ 6.93\end{array}$

$\begin{array}{llll}4.0 & 302.1 & 3.40 & 2.02\end{array}$ $\begin{array}{llll}2.0 & 300.3 \quad 3.36 \quad 1.39\end{array}$

SOIL TEMPERATURES $24.4 \quad 26.4 \quad 27.5$

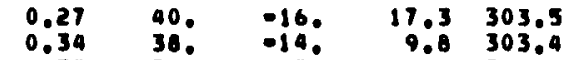
NET RADIATIONE - -30.WIMER

DAY 183 STARTING TIME 22310124

$v$
1.54
1.31
1.32
0.95
0.65
27.3

$\begin{array}{lllll}W & \text { TH } & \text { PHI } & \text { HT } & \text { POT T } \\ 0.31 & 24 . & -16 . & 17.3 & 303.5 \\ 0.35 & 21 . & -15 . & 9.8 & 303.4 \\ 0.33 & 20 . & -16 . & 6.0 & 301.3 \\ 0.30 & 19 . & -16 . & 2.1 & 299.8 \\ 0.04 & 12 . & -20 . & 1.4 & 298.1 \\ \text { NET RADIATION= } & -30 . N / M \# * 2 & \end{array}$

$\begin{array}{ccc}u & v & w \\ 2.65 & 3.01 & 0.25 \\ 3.30 & 2.71 & 0.32 \\ 3.73 & 1.95 & 0.32 \\ 3.17 & 1.17 & 0.12 \\ 2.36 & 0.45 & 0.83\end{array}$

IN PHI

$\begin{array}{ll}3.8 & 302.5 \\ 3.8 & 302.4\end{array}$

2.0299 .9

$9.1303 .3 \quad 3.47$

$\begin{array}{lll}6.0 & 303.0 & 3.37 \\ 4.0 & 302.6 & 3.59\end{array}$

$\begin{array}{llll}2.0 & 300.1 & 3.72 & 0.95 \\ 1.2 & 299.2 & 3.07 & 0.065\end{array}$

SOIL TEMPERATURES:

24.2

6.2

NET RADIATION= $=-30, N / M * * 2$ 
JULY 1-2, 1980: 1920-0200 PST DAY TOWER STARTING TIME 52850154

\begin{tabular}{|c|c|c|c|c|c|c|}
\hline u & v & $\mathbf{w}$ & TH & PHI & HT & TOT \\
\hline $\begin{array}{l}3.59 \\
3.48 \\
3.59 \\
3.80 \\
3.13\end{array}$ & $\begin{array}{l}0.84 \\
0.59 \\
0.69 \\
0.61 \\
0.43\end{array}$ & $\begin{array}{l}0.31 \\
0.31 \\
0.31 \\
0.31 \\
0.05\end{array}$ & $\begin{array}{l}13 . \\
10 . \\
11 . \\
90 \\
8\end{array}$ & $\begin{array}{l}-16 . \\
-15 . \\
-16 . \\
-16 . \\
-20 .\end{array}$ & $\begin{array}{r}17.3 \\
9.8 \\
6.0 \\
2.7 \\
1.4\end{array}$ & $\begin{array}{l}303.6 \\
303.5 \\
301.5 \\
298.9 \\
298.3\end{array}$ \\
\hline
\end{tabular}

TOWER C

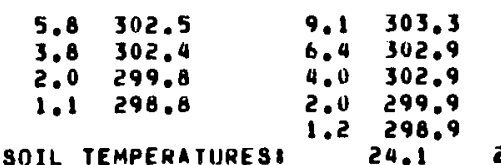

DAY 183 STARTING TIME 22830129
HT POT T POT T

\begin{tabular}{|c|c|c|c|c|c|}
\hline $\begin{array}{l}5.8 \\
3.8 \\
2.0 \\
1.1\end{array}$ & $\begin{array}{l}302.5 \\
302.3 \\
299.7 \\
298.7\end{array}$ & $\begin{array}{l}9.1 \\
6.4 \\
4.0 \\
2.0 \\
1.2\end{array}$ & $\begin{array}{l}303.3 \\
303.0 \\
302.0 \\
299.9 \\
299.1 \\
23.9\end{array}$ & $\begin{array}{r}3.47 \\
3.40 \\
3.66 \\
3.16 \\
3.09 \\
25.8\end{array}$ & $\begin{array}{c}-0.15 \\
-0.52 \\
-0.05 \\
0.30 \\
0.04 \\
26.9\end{array}$ \\
\hline
\end{tabular}

$\begin{array}{ccccc}W & T H & P H I & H T & P O T \\ 0.32 & -2 . & -16 . & 17.3 & 303.5 \\ 0.39 & -9 . & -14 . & 9.8 & 303.5 \\ 0.33 & -7 . & -16 . & 6.0 & 301.4 \\ 0.31 & 5 . & -16 . & 2.7 & 290.8 \\ 0.05 & 1 . & -20 . & 1.4 & 298.2\end{array}$

$\begin{array}{cc}u & v \\ 4.08 & 1.33 \\ 4.24 & 0.99 \\ 4.55 & 0.33 \\ 3.62 & 0.34 \\ 2.63 & 0.72\end{array}$

$\begin{array}{ccc} & T H & P H I \\ 0.25 & 10 . & -5 . \\ 0.24 & 13 . & -5 . \\ 0.38 & 40 & -3 . \\ 0.16 & 50 & -6 . \\ 0.58 & 15 . & 4 .\end{array}$
NET RADIATION= -29,W/MHE

DAY 183 STARTING TIME 22140124

$\begin{array}{ll}H .8 & 302.5 \\ 5.8 & 302.3 \\ 2.0 & 299.9 \\ 1.1 & 298.9\end{array}$

HT POT $T$

$\begin{array}{cc}u & v \\ 3.11 & -0.25 \\ 3.15 & -0.67 \\ 3.63 & -0.67 \\ 3.76 & 9.99 \\ 3.09 & -0.15 \\ 25.7 & 26.0\end{array}$

W TH PHI HT POT T

$0.30 \quad-5 . \quad-15 . \quad 87.3 \quad 303.7$

$\begin{array}{lllll}0.40 & -12 . & -14 . & 9.8 & 303.5 \\ 0.34 & -10 . & -15 . & 6.0 & 301.3\end{array}$

$0.34 \quad 999 . \quad-16.0 \quad 2.7298 .9$

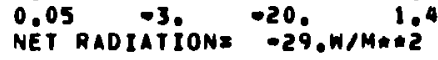

298.9

$\begin{array}{ll}4.0 & 302.7 \\ 2.0 & 300.3\end{array}$

2299.

DAY 183 STARTING TIME 22850824
HT POT.T HT POT T

$\begin{array}{ll}5.8 & 302.6 \\ 3.8 & 302.4 \\ 2.0 & 300.0\end{array}$

$\begin{array}{ll}2.0 & 300.0 \\ 1.1 & 298.8\end{array}$

SOIL TEMPEAATURES!

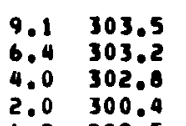

$2.0 \quad 300.4$

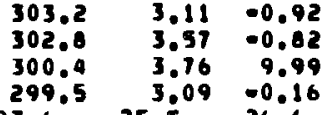

26.6

$\begin{array}{ccccc}W & T H & P H I & H T & \text { POT } \\ 0.30 & -11 . & -16 . & 17.3 & 303.7 \\ 0.38 & -17 . & -19 . & 9.8 & 303.6 \\ 0.34 & -13 . & -15 . & 6.0 & 301.1 \\ 0.34 & 999 . & -16 . & 2.7 & 299.1 \\ 0.05 & -3 . & -20 . & 1.4 & 290.4\end{array}$

$\begin{array}{ccccc}U & V & W & P H & P H I \\ 4.02 & 0.48 & 0.28 & 7 . & -4 . \\ 9.17 & -0.12 & 0.23 & -2 . & -5 . \\ 4.48 & -0.36 & 0.39 & -5 . & -3 . \\ 3.59 & -0.03 & 0.16 & -0.0 & -6 . \\ 2.66 & 0.65 & 0.40 & 14 . & 0 .\end{array}$

$\begin{array}{ccccc}U & V & W & T H & \text { PHI } \\ 3.35 & 0.32 & 0.33 & 5 . & -3 . \\ 3.75 & -0.23 & 0.02 & -3 . & -5 . \\ 4.17 & -0.07 & 0.42 & -6 . & -3 . \\ 3.42 & -0.09 & 0.14 & -2 . & -6 . \\ 2.57 & 0.62 & 0.34 & 14 . & -1 .\end{array}$

$$
\begin{array}{ll}
\text { HT } & \text { POT T } \\
5.8 & 302.6 \\
3.8 & 302.6 \\
2.0 & 300.4 \\
1.1 & 298.8
\end{array}
$$

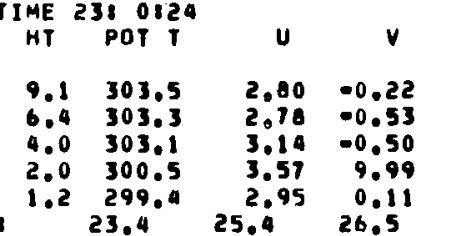

NET RADIATIONE -29,W/MMEZ

SOIL TEMPERATURES

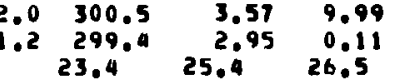

$\begin{array}{ccccc}W & T H & \text { PHI } & \text { HT } & \text { POT T } \\ 0.28 & -4 . & -15 . & 17.3 & 303.4 \\ 0.36 & -11 . & -13 . & 9.8 & 303.2 \\ 0.31 & -9 . & -15 . & 6.0 & 301.0 \\ 0.34 & 999 . & -15 . & 2.7 & 298.9 \\ 0.06 & 2 & -20 . & 1.9 & 298.2\end{array}$
0.06 999. $\quad-150 \quad 2.7298 .9$

\begin{tabular}{|c|c|c|c|c|c|}
\hline${ }_{H Y}{ }_{H}^{103}$ & $\begin{array}{l}3 \text { STARTING } \\
\text { POT T }\end{array}$ & $\begin{array}{c}\text { TIME } \\
\text { HI }\end{array}$ & $\begin{array}{l}23110125 \\
\text { POI T }\end{array}$ & U & $v$ \\
\hline $\begin{array}{l}5.0 \\
3.0 \\
2.0 \\
1.1\end{array}$ & $\begin{array}{l}302.6 \\
302.6 \\
300.6 \\
298.6\end{array}$ & $\begin{array}{l}9.1 \\
6.4 \\
4.0 \\
2.0 \\
1.2\end{array}$ & $\begin{array}{r}303.5 \\
303.3 \\
303.0 \\
300.8 \\
299.3 \\
23.2\end{array}$ & $\begin{array}{l}1.99 \\
1.98 \\
2.47 \\
3.25 \\
2.17 \\
25.2\end{array}$ & $\begin{array}{r}-0.30 \\
-0.54 \\
-0.06 \\
9.99 \\
0.05 \\
26.3\end{array}$ \\
\hline
\end{tabular}
NET RADIATIONE $=20$. $=29, W / M=1.4$

DAY 103 STARTING TIME 23110125

$\begin{array}{rrrrr}H & T H & \text { PHI } & \text { HT } & \text { POT T } \\ 0.22 & -8 . & -15 . & 17.3 & 302.1 \\ 0.29 & -15 . & -12 . & 9.8 & 301.8 \\ 0.26 & -10 . & -15 . & 6.0 & 301.0 \\ 0.38 & 999 . & -14 . & 2.7 & 299.9 \\ 0.07 & 1 . & -19 . & 1.4 & 298.8\end{array}$

$\begin{array}{ccccc}U & V & W & T H & P H I \\ 3.24 & 0.32 & 0.37 & 6 . & -2 . \\ 3.63 & -0.26 & 0.26 & -4 . & -4 . \\ 3.97 & -0.39 & 0.39 & -6 . & -3 . \\ 3.25 & 0.11 & 0.15 & 2 . & -6 . \\ 2.06 & 0.68 & 0.46 & 15 . & 2 .\end{array}$
$\begin{array}{ccc}23.2^{2.3} & 25.2^{2.17} & 0.05 \\ & & \end{array}$

$0.07 \quad 1: \quad-19 . \quad 1.4298 .9$

$\begin{array}{ccccc}U & V & W & T H & \text { PHI } \\ 1.61 & 1.08 & 0.39 & 34 . & 5 . \\ 2.29 & 0.64 & 0.33 & 16 . & 0 . \\ 2.92 & 0.47 & 0.49 & 9 . & 1 . \\ 2.55 & 0.06 & 0.17 & 19 . & 14 . \\ 1.09 & 0.80 & 0.69 & 23 . & 12 .\end{array}$

$\begin{array}{ccc}u & v & w \\ 0.70 & 1.81 & 0.15 \\ 1.27 & 1.45 & 0.18 \\ 1.65 & 1.10 & 0.43 \\ 2.10 & 1.01 & 0.20 \\ 1.84 & 0.76 & 0.67\end{array}$

$\begin{array}{ll}\text { TH } & P H I \\ 69 . & 4 . \\ 49 . & -0 . \\ 34 . & 6 . \\ 26 . & -3 . \\ 23 . & 12 .\end{array}$ 
JULY 1-2, 1980; 1920-0200 PSI

TOWER Â

DAY 103 STAKIING TIME 23820825

IOWEH B

TOWER C

$\begin{array}{lllllllllrl}5.8 & 302.5 & 9.1 & 303.4 & 2.01 & -0.02 & 0.22 & -1 . & -15 . & 17.3 & 302.0 \\ 3.8 & 302.4 & 6.4 & 303.2 & 1.99 & -0.40 & 0.29 & -11 . & -12 . & 9.8 & 301.08 \\ 2.0 & 300.5 & 4.0 & 302.9 & 2.46 & -0.35 & 0.27 & -8 . & -15 . & 6.0 & 300.9 \\ 1.1 & 298.5 & 2.0 & 300.7 & 3.28 & 9.99 & 0.40 & 999 . & -14 . & 2.7 & 299.3 \\ & & 1.2 & 299.0 & 2.85 & 0.17 & 0.06 & 4 . & -20 . & 1.4 & 297.8\end{array}$

$\begin{array}{ccccr}U & V & N & T H & P H 1 \\ 1.11 & 1.58 & 0.16 & 55 . & 0 . \\ 1.46 & 1.06 & 0.21 & 36 . & -0 . \\ 1.02 & 0.72 & 0.43 & 22 . & 5 . \\ 2.41 & 0.78 & 0.10 & 18 . & -4 . \\ 2.10 & 0.71 & 0.56 & 19 . & 7 .\end{array}$

23130825

OAY 183 SIARTING TIME 23330825

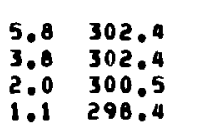

SOIL TEMPERATURES,

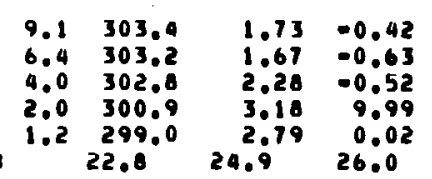

$22.0^{\circ} 24.9$
$W$ TH PHI HT POT T

$0.21 \quad-14, \quad-140 \quad 17.3301 .9$

$0.28 \quad-21 . \quad-11.0 \quad 9.8301 .5$

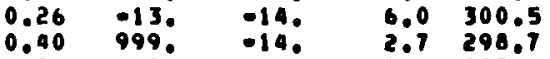
$0.08 \quad 00 \quad-19, \quad \begin{array}{lll}1.9 & 298.4\end{array}$ NET RADIATIONa - 27 .WIMME

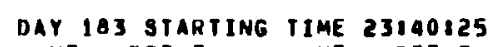

SOIL TEMPERATURESI

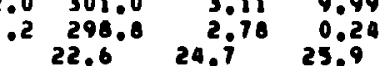

DAY 183 STARTING TIME 23150825

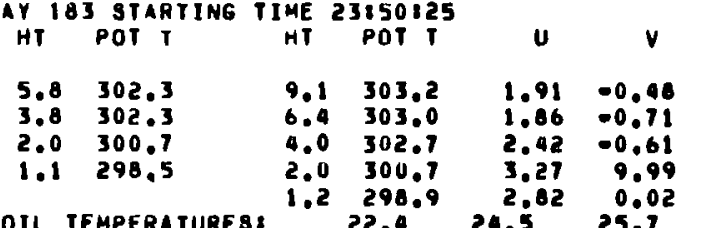

SOIL TEMPERATURES:

$22.4 \quad 24.5 \quad 25.7$

$0.22-10 . \quad-14 . \quad 17.3301 .9$

$0.28-16 . \quad-12 . \quad 9.8301 .7$

$0.20 \quad-10, \quad-15, \quad 6.0300 .9$

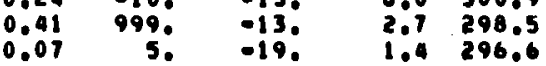
NET RADIATIONE - -26. W/MEAC

W TH PHI HT POT

$\begin{array}{lllll}0.23 & -14 . & -14 . & 17.3 & 302.1 \\ 0.30 & -21: & -12 . & 9.8 & 302.0\end{array}$

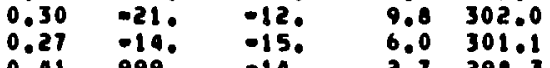

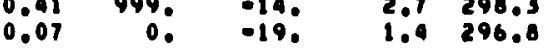
NET RADIATIONE -26.W/MERZ

DAY 104 STARTING TIME O\&OIZ5

$\begin{array}{llllll}5.8 & 302.4 & 9.1 & 303.1 & 2.68 & -1.39 \\ 3.6 & 302.0 & 6.4 & 302.8 & 2.65 & -1.59 \\ 2.0 & 299.9 & 4.0 & 302.2 & 3.32 & -1.24 \\ 1.1 & 298.7 & 2.0 & 300.3 & 3.52 & 9.99 \\ & 1.2 & 299.3 & 2.91 & -0.24\end{array}$

SOIL TEMPERATURES:

DOU TOU STARTING TIME

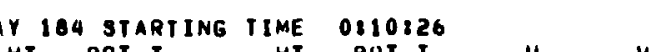

W TH PHI HT POT T

$\begin{array}{lll}0.02 & 1.29 & 0.12 \\ 1.29 & 0.78 & 0.16\end{array}$

$1.29 \quad 0.78 \quad 0.16$

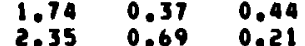

$\begin{array}{lll}2.35 & 0.69 & 0.21 \\ 2.01 & 0.73 & 0.62\end{array}$

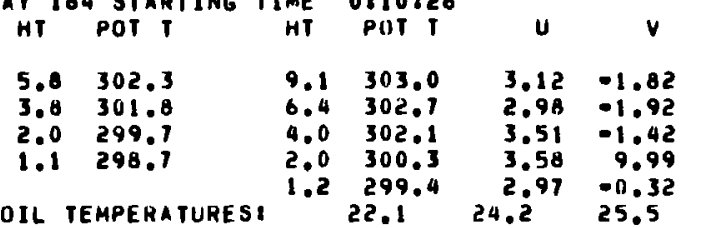

$0.32 \quad-27, \quad-14 . \quad 17.3 \quad 302.6$

0.39 -31. -13. 9.0302 .4

$0.34 \quad-21 . \quad-15 . \quad 6.0301 .5$

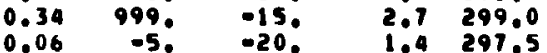
NET RADIATIONA $-20.6 / M A M$

$\begin{array}{ccccr}U & V & W & T H & P H I \\ 0.18 & 0.60 & 0.22 & 38 . & 7 . \\ 1.30 & 0.01 & 0.21 & 1: & 1: \\ 1.17 & -0.35 & 0.56 & -11: & 9 . \\ 2.59 & 0.00 & 0.20 & 0 . & -4: \\ 2.14 & 0.63 & 0.34 & 16 . & 1 .\end{array}$

W TH PHI

0.36 T30.

$\begin{array}{rrrrr}0.36 & -30, & -14 . & 17.3 & 302.5 \\ 0.41 & -33 . & -13 . & 9.8 & 302.7 \\ 0.35 & -22 . & -15 . & 6.0 & 302.0\end{array}$

$0.35 \quad-22 . \quad-15 . \quad 6.0302 .0$

$0.06 \quad-6 . ; \quad-20 . \quad 1.4298 .7$

NET RADIATION= $=-20.25$. W/ME

$\begin{array}{ccccc}U & V & W & T H & P H I \\ 0.09 & 0.58 & 0.15 & 33 & 1 . \\ 1.25 & 0.27 & 0.24 & 12 . & 3 . \\ 1.77 & -0.11 & 0.53 & -3 . & 8 . \\ 2.57 & 0.37 & 0.19 & 0.0 & -4 . \\ 2.11 & 0.69 & 0.55 & 10 . & 6 .\end{array}$

SOIL TEMPERATURES: 22,1

-

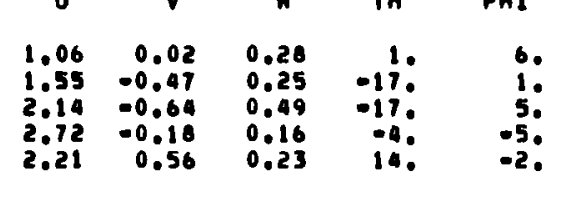

$\begin{array}{ccccc}U & V & W & T H & P H I \\ 0.25 & 0.01 & 0.01 & 3 . & -5 . \\ 1.06 & -0.60 & 0.12 & -29 . & -2 . \\ 1.81 & -0.82 & 0.37 & -24 . & 3 . \\ 2.31 & -0.28 & 0.11 & -7 . & -6 . \\ 2.00 & 0.34 & 0.11 & 10 . & -5 .\end{array}$ 
JULY 1-2, 1980: 1920-0200 PST

TOWER

DAY 184 STARIING IIME O\&20\$5

1OWEH H

TOWER C

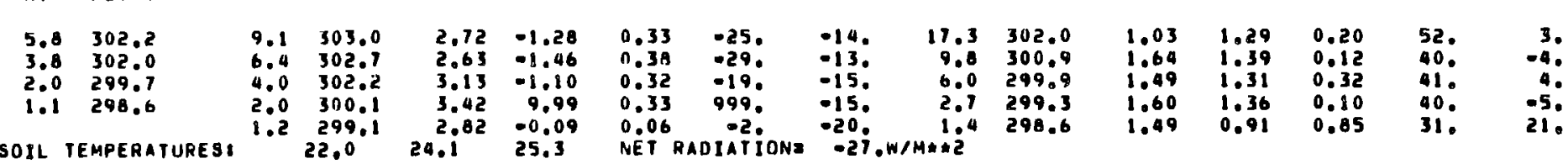

SOIL TEMPERATURESI

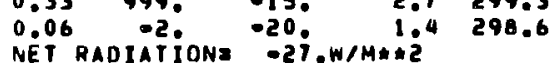

DAY 184 STARTING TIME OB30826

$\begin{array}{ll}5.8 & 302.1 \\ 3.0 & 302.1 \\ 2.0 & 299.5\end{array}$

2.0299 .5

$\begin{array}{rrrr}9.1 & 302.9 & 1.83 & -0.67 \\ 6.4 & 302.7 & 1.77 & -0.86 \\ 4.0 & 302.1 & 2.56 & 0.062 \\ 2.0 & 299.8 & 3.24 & 9.99 \\ 1.2 & 298.5 & 2.75 & 0.29 \\ & 21.9 & 23.9 & 25.2\end{array}$

W TH PHI HT POT

$\begin{array}{lllll}U & V & \text { W } & \text { TH } & \text { PHI }\end{array}$

SOIL TEMPERATURES:

$0.25 \quad-20, \quad-13 . \quad 17.3 \quad 300.9$

$0.31 \quad-26 . \quad-11 . \quad 9.8 \quad 300.7$

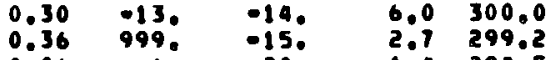

$0.06 \quad 6.020 . \quad 1.4296 .5$

$\begin{array}{llll}1.72 & 3.07 & 0.32 & 61 \\ 1.89 & 2.41 & 0.40 & 52.0\end{array}$

$1.892 .41 \quad 0.40 \quad 52$.

$\begin{array}{llll}1.82 & 1.85 & 0.47 & 45 \\ 1.01 & 1.46 & 0.19 & 39\end{array}$

OAY 1B4 STARTING TIME OIAOIZ6
HT POT T POT T

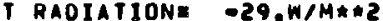

$\begin{array}{llllll}5.8 & 302.0 & 9.1 & 302.9 & 2.56 & -1.03 \\ 3.8 & 301.8 & 6.4 & 302.6 & 2.49 & -1.26 \\ 2.0 & 299.3 & 4.0 & 302.1 & 3.12 & -1.00 \\ 1.1 & 298.3 & 2.0 & 299.7 & 3.45 & 9.99 \\ \text { SOIL TEMPERATURESI } & 1.2 & 298.7 & 2.85 & -00.25 \\ & & 21.7 & 23.8 & 25.1\end{array}$

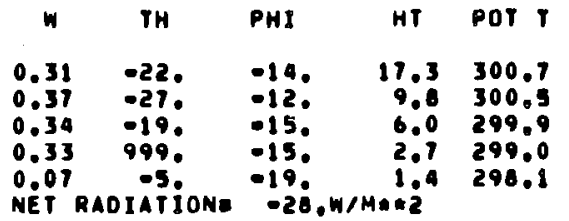

$\begin{array}{ccccc}U & V & W & \text { YH } & \text { PHI } \\ 1.02 & 2.31 & 0.32 & 66 . & 9 . \\ 1.35 & 1.54 & 0.34 & 49 . & 6 . \\ 1.50 & 1.12 & 0.47 & 37 . & 9 . \\ 1.65 & 0.97 & 0.24 & 31 . & -0 . \\ 1.45 & 0.79 & 0.67 & 28 . & 17 .\end{array}$

DAY 164 STARTING TIME O OI5O126 NET RAOIATION= -198, WMMER

$1.65 \quad 0.97 \quad 0.24 \quad 31.0$

$\begin{array}{lll}5.8 & 301.9 & 9.9 \\ 3.8 & 301.6 & 6 \\ 2.0 & 299.2 & 4.0 \\ 1.1 & 298.2 & 2.0\end{array}$

9.1302 .8

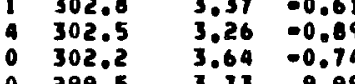

$.0299 .5 \quad 3.73 \quad 9.99$

SOIL TEMPERATURESI

21.6

$3.04 \quad-0.12$

w TH PHI HY POT T

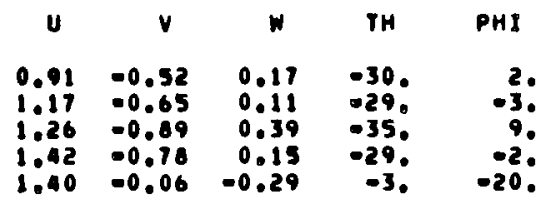

DAY 184 STARTING TIME 11,0827

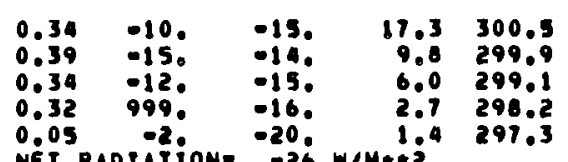

$1.40-0.78 \quad 0.15$

NET RAOLATIONE -26,W/MERE

$\begin{array}{rrrrrr}5.8 & 301.4 & 9.1 & 302.3 & 3.92 & -0.31 \\ 3.8 & 3011.1 & 6.4 & 301.9 & 3.87 & -0.53 \\ 2.0 & 299.0 & 4.0 & 301.4 & 4.15 & -0.30 \\ 1.1 & 298.1 & 2.0 & 299.1 & 3.07 & 9.99 \\ & 1.2 & 298.3 & 3.13 & 0.06 \\ \text { SOIL TEMPERATURES } & & 21.4 & 23.5 & 24.8\end{array}$
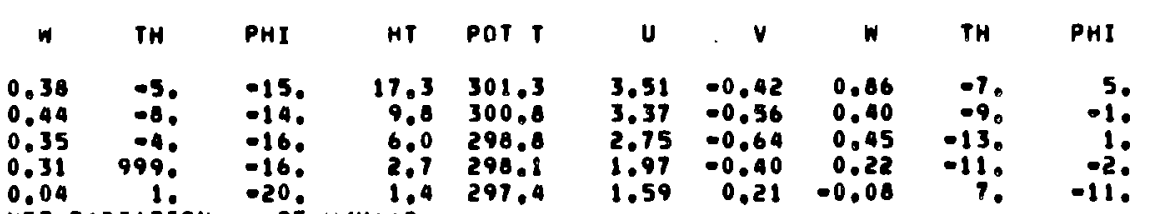
DAY 104 STARTING TIME NET RADIATIONE -25.W/MHAR

$1.59 \quad 0.21 \quad-0.00$

W TH PHI HT POT

$\begin{array}{ccccc}U & V & W & \text { IH } & \text { PHI } \\ 4.16 & -0.12 & 0.60 & -1 . & -1 . \\ 4.90 & -0.13 & 0.32 & -1 . & -4 . \\ 4.01 & -0.14 & 0.36 & -2 . & -4 . \\ 3.60 & 0.28 & 0.16 & 4 . & -6 . \\ 2.66 & 0.71 & 0.54 & 15 . & 3 .\end{array}$

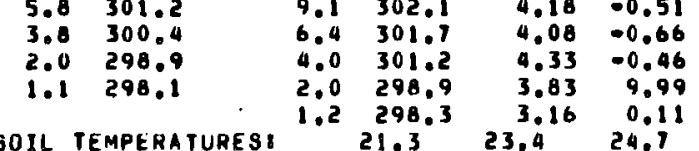

$0.39 \quad-1 . \quad-16 . \quad 17.3302 .4$

$\begin{array}{lllll}0.44 & -9 . & -15 . & 9.8 & 302.2 \\ 0.36 & -6 . & -16 . & 6.0 & 299.3\end{array}$

0.30 999. -16. $\quad 2.7298 .0$

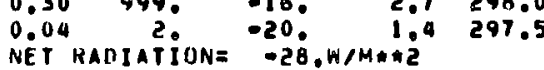


$\underline{\text { JULY 1-2. 1980; 1920-0200 PST }}$

$$
\text { TOWER A }
$$

DAY IOA STARTING TIME $1: 20: 20$

HT POT T

u

TOWEN H

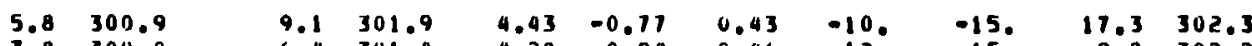

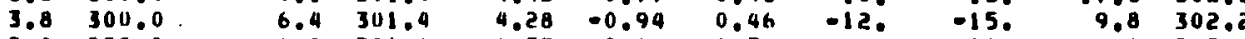

$2.0 \quad 298.8$

$4.53 \quad-0.61$

1.1 29h.

$2.0 \quad 298.9$

$3.82 \quad 9.99$

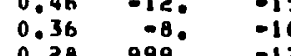

SOIL TEMPERATURESI

298.4

24.5

DAY 184 STARTING TIME

1,30151

$\begin{array}{lll}0.28 & 999 . & -17: \\ 0.03 & -2 . & -20 .\end{array}$

$\begin{array}{ll}6.0 & 299.6 \\ 2.7 & 298.4 \\ 1.4 & 291.9\end{array}$

HT 1830127
PITT

$\begin{array}{ll}5.8 & 300.7 \\ 3.8 & 299.9\end{array}$

2.0290 .8

$\begin{array}{llll}9.1 & 301.9 & 4.58 & -0.9 \\ 6.4 & 301.4 & 4.46 & -0.4 \\ 4.0 & 300.3 & 4.61 & -0.2 \\ 2.0 & 298.9 & 3.84 & 9.9\end{array}$

SOIL TEMPERATURES:

$\begin{array}{llll}9.0 & 298.9 & 3.84 & 9.99\end{array}$

$21.3^{298.4} 23.2^{3.13}$

$v$
-0.12
-0.40
-0.20
9.99
0.16
24.4

$\begin{array}{lllll}W & T H & P H I & H T & P 0 T \\ 0.42 & -2 . & -16 . & 17.3 & 302.3 \\ 0.45 & -5 . & -15 . & 9.8 & 302.2 \\ 0.34 & -3 . & -17 . & 6.0 & 299.6 \\ 0.27 & 999 . & -17 . & 2.7 & 298.4 \\ 0.03 & 3 . & -20 . & 1.4 & 298.0\end{array}$

IOWER C

OAY 184 STARTING TIME 1840827
HT POTT

5.8301 .0

2.0299 .4

1.1299 .0

$\ddot{w}$

SOIL TEMPERATURESI

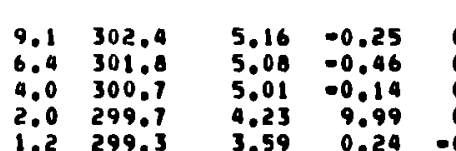

W TH PHI HT POT T

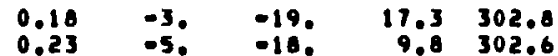

$\begin{array}{lllll}0.23 & -5 . & -18 . & 9.8 & 302.6 \\ 0.03 & -2.0 & -21: & 6.0 & 300.2 \\ 0.03 & 9990 & -20 . & 2.7 & 299.3\end{array}$

DAY 184 STARTING TIME

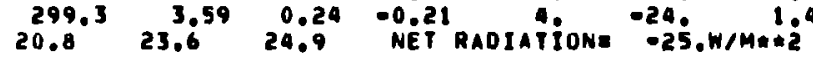

$\begin{array}{llllll}\text { DAT POT T } & \text { HT } & \text { POT T } & U & V \\ 5.8 & 300.6 & 9.1 & 301.8 & 4.41 & -0.62 \\ 3.8 & 299.9 & 6.4 & 301.3 & 4.33 & -0.01 \\ 2.0 & 299.0 & 4.0 & 300.3 & 4.33 & -0.54 \\ 1.1 & 298.6 & 2.0 & 299.3 & 3.58 & 9.99 \\ \text { SOIL TEMPERATURESI } & 1.2 & 298.9 & 2.93 & -0.013 \\ & & 21.4 & 23.0 & 24.1\end{array}$

$\begin{array}{ccccc}W & \text { TH } & \text { PHI } & \text { HT } & \text { POT T } \\ 0.42 & -8 . & -15 . & 17.3 & 302.2 \\ 0.46 & -11 . & -15 . & 9.8 & 302.1 \\ 0.31 & -7 . & -17 . & 6.0 & 299.7 \\ 0.27 & 999 . & -16 . & 2.7 & 298.8 \\ 0.06 & -3 . & -20 . & 1.44 & 298.4\end{array}$

$\begin{array}{ccccc}U & V & W & T H & P H I \\ 5.16 & -0.56 & 0.46 & -6 . & -3 . \\ 5.21 & -0.59 & 0.29 & -6 . & -5 . \\ 4.93 & -0.50 & 0.37 & -6 . & -4 . \\ 3.75 & -0.11 & 0.16 & -2 . & -6 . \\ 2.81 & 0.58 & 0.32 & 12 . & -2 .\end{array}$

U. $V$ TH PHI

$5.49-0.19 \quad 0.34 \quad-2 . \quad-5$.

$\begin{array}{rrrrr}5.64 & -0.14 & 0.30 & -1 . & -5 . \\ 5.42 & -0.19 & 0.40 & -2 . & -4 . \\ 4.20 & 0.14 & 0.19 & 2 . & -6 . \\ 3.06 & 0.71 & 0.57 & 13 . & 2 .\end{array}$

SOIL TEMPERATURES:

21.4 .932 .0

1 NET RADIATION= -20:

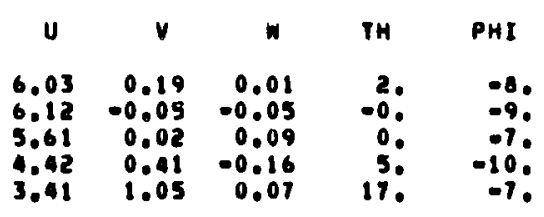

$\begin{array}{ccccc}U & V & W & \text { IH } & P H I \\ 5.35 & -0.19 & 0.35 & -2 . & -5 . \\ 5.39 & -0.32 & 0.30 & -3 . & -5 . \\ 5.00 & -0.35 & 0.41 & -4 . & -4 . \\ 3.89 & 0.02 & 0.17 & 0 . & -6 . \\ 2.88 & 0.68 & 0.49 & 13 . & 1 .\end{array}$


JULY 2-3, 1981: 1813-0345 PST

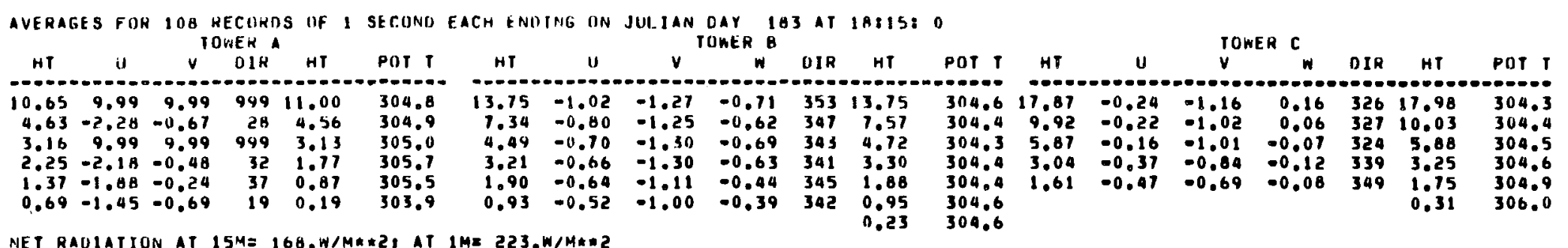

NET RAUIATION AT 15M=160.W/MA*2, AT IME 223. W/MHELC

AVERAGES FOR 900 RECBROS OF 1 SECOND EACH ENOING ON JULLAN DAY 103 AT 1883010

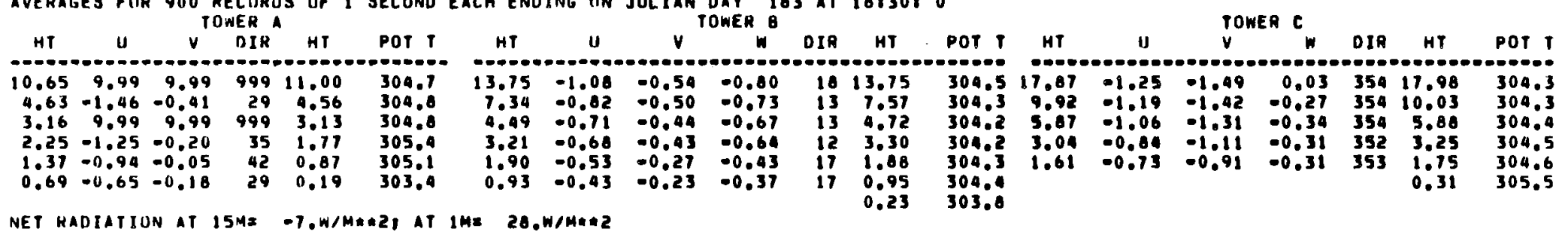

AVERAGES FUR 9DO RECORDS OF 1 SECOND EACH ENDING ON JULIAN DAY 103 AT 1014580

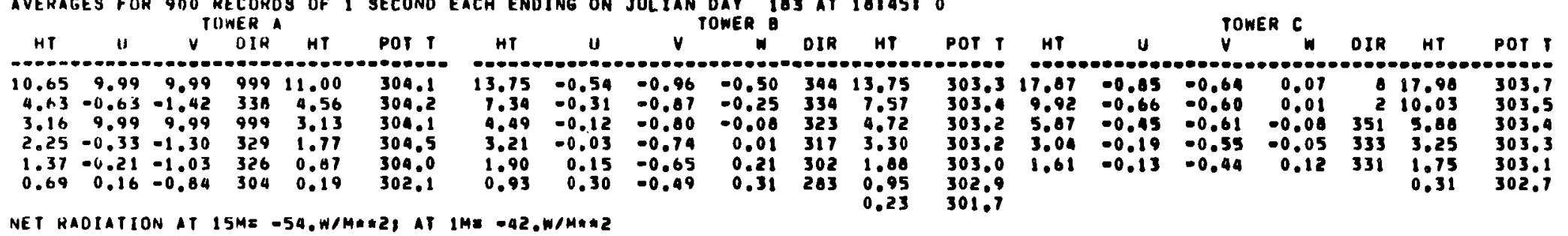

NET RAOIATION AT 15ME -54,WIMARE, AT IME -42,W/MAWZ

AVERAGES FOR 900 KECUROS OF 1 SECOND EACH ENUING UN JULIAN DAY 183 AT $1920: 0$

\begin{tabular}{|c|c|c|c|c|c|c|c|c|c|c|c|c|c|c|c|c|c|c|c|}
\hline HT & u & $v$ & OIA & HT & Por $T$ & HT & $u$ & $v$ & $w$ & DIR & HT & POT $T$ & HT & u & $v$ & $m$ & DIR & HT & POT T \\
\hline $\begin{array}{r}10.65 \\
4.63 \\
3.16 \\
2.25 \\
1.37 \\
0.69\end{array}$ & $\begin{array}{r}9.99 \\
-0.110 \\
9.99 \\
0.20 \\
0.18 \\
0.54\end{array}$ & $\begin{array}{r}9.99 \\
-1.96 \\
9.99 \\
-1.84 \\
-1.50 \\
-1.07\end{array}$ & $\begin{array}{l}999 \\
317 \\
999 \\
308 \\
308 \\
288\end{array}$ & $\begin{array}{r}11.00 \\
4.56 \\
3.13 \\
1.17 \\
0.87 \\
0.19\end{array}$ & $\begin{array}{l}304.0 \\
304.1 \\
303.9 \\
304.2 \\
303.4 \\
301.6\end{array}$ & $\begin{array}{r}13.15 \\
7.34 \\
4.49 \\
3.21 \\
1.90 \\
0.93\end{array}$ & $\begin{array}{r}-0.19 \\
0.24 \\
0.51 \\
0.66 \\
0.83 \\
0.71\end{array}$ & $\begin{array}{l}-2.23 \\
-2.03 \\
-1.89 \\
-1.68 \\
-1.53 \\
-0.91\end{array}$ & $\begin{array}{r}-0.02 \\
0.42 \\
0.59 \\
0.74 \\
0.87 \\
0.84\end{array}$ & $\begin{array}{l}319 \\
308 \\
299 \\
293 \\
286 \\
278\end{array}$ & $\begin{array}{r}13.15 \\
7.57 \\
4.72 \\
3.30 \\
1.88 \\
0.95 \\
0.23\end{array}$ & $\begin{array}{l}302.8 \\
303.2 \\
302.9 \\
302.8 \\
302.3 \\
302.1 \\
301.1\end{array}$ & $\begin{array}{r}17.07 \\
9.92 \\
5.87 \\
3.04 \\
1.61\end{array}$ & $\begin{array}{l}0.97 \\
0.91 \\
1.27 \\
1.35 \\
0.90\end{array}$ & $\begin{array}{l}-2.13 \\
-2.09 \\
-1.96 \\
-1.49 \\
-1.07\end{array}$ & $\begin{array}{l}0.20 \\
0.45 \\
0.42 \\
0.46 \\
0.72\end{array}$ & $\begin{array}{l}302 \\
291 \\
202 \\
272 \\
272\end{array}$ & $\begin{array}{l}17.98 \\
10.03 \\
5.88 \\
3.25 \\
1.75 \\
0.31\end{array}$ & $\begin{array}{l}303.2 \\
302.9 \\
302.5 \\
302.0 \\
3011.4 \\
301.0\end{array}$ \\
\hline
\end{tabular}

NET RADIATION AT ISHE= -54,W/M*\#2, AT $1 M=-44, W / M \# 2$ 
JULY 2-3. 1981; 1813-0345 PST

\begin{tabular}{|c|c|c|c|c|c|c|c|c|c|c|c|c|c|c|c|c|c|c|}
\hline $\begin{array}{l}\text { AVERAGS. } \\
\text { HT }\end{array}$ & $S F \mathrm{FH}$ & $\mathrm{v}^{900 \mathrm{~K}}$ & $\begin{array}{l}\text { ECDKD } \\
\text { AERAA } \\
\text { DIR }\end{array}$ & $\begin{array}{l}\text { DS OF I } \\
\text { HT }\end{array}$ & $\begin{array}{l}\text { SECOIHD } \\
\text { POT T }\end{array}$ & $\begin{array}{c}\text { EACH ENDINS; } \\
\text { HT }\end{array}$ & II ON & $\begin{array}{c}\text { JULIAN DAY } \\
\text { TOWER B }\end{array}$ & $\begin{array}{l}83 A T \\
\text { DIR }\end{array}$ & $\begin{array}{c}198151 \\
\text { HT }\end{array}$ & PDI & HT & u & $V_{V}^{T O}$ & $c_{w}$ & DIR & HT & TOT \\
\hline $\begin{array}{l}10.65 \\
4.63 \\
3.16 \\
2.25 \\
1.37 \\
0.69\end{array}$ & $\begin{array}{l}9.99 \\
0.21 \\
9.99 \\
0.55 \\
0.39 \\
0.71\end{array}$ & $\begin{array}{r}9.94 \\
-2.33 \\
9.99 \\
-2.06 \\
-1.65 \\
-1.11\end{array}$ & $\begin{array}{l}999 \\
309 \\
999 \\
300 \\
301 \\
2 B 2\end{array}$ & $\begin{array}{r}11.00 \\
4.56 \\
3.13 \\
1.71 \\
0.87 \\
0.19\end{array}$ & $\begin{array}{l}303.0 \\
303.8 \\
303.6 \\
303.9 \\
303.0 \\
301.4\end{array}$ & $\begin{array}{r}13.75 \\
1.34 \\
4.49 \\
3.21 \\
1.90 \\
0.93\end{array}$ & $\begin{array}{l}0.12 \\
0.57 \\
0.86 \\
0.97 \\
1.08 \\
0.87\end{array}$ & $\begin{array}{l}-2.53 \\
-2.32 \\
-2.16 \\
-1.81 \\
-1.65 \\
-0.94\end{array}$ & $\begin{array}{l}312 \\
301 \\
293 \\
286 \\
281 \\
272\end{array}$ & $\begin{array}{r}13.75 \\
7.57 \\
4.72 \\
3.30 \\
1.88 \\
0.95 \\
0.23\end{array}$ & $\begin{array}{l}302.5 \\
302.9 \\
302.6 \\
302.4 \\
301.9 \\
301.0 \\
3000.8\end{array}$ & $\begin{array}{r}17.87 \\
9.92 \\
5.87 \\
3.04 \\
1.61\end{array}$ & $\begin{array}{l}1.00 \\
1.43 \\
1.73 \\
1.60 \\
1.06\end{array}$ & $\begin{array}{l}-2.76 \\
-2.55 \\
-2.40 \\
-1.70 \\
-1.27\end{array}$ & $\begin{array}{l}0.37 \\
0.59 \\
0.57 \\
0.55 \\
0.79\end{array}$ & $\begin{array}{l}295 \\
285 \\
279 \\
271 \\
275\end{array}$ & $\begin{array}{r}17.98 \\
10.03 \\
5.88 \\
3.25 \\
1.75 \\
0.31\end{array}$ & $\begin{array}{l}303.1 \\
302.8 \\
302.3 \\
301.6 \\
301.1 \\
300.9\end{array}$ \\
\hline
\end{tabular}

NET RADIAIION AT 15ME -54,W/M**2, AT IM= $-44, W / M A 42$

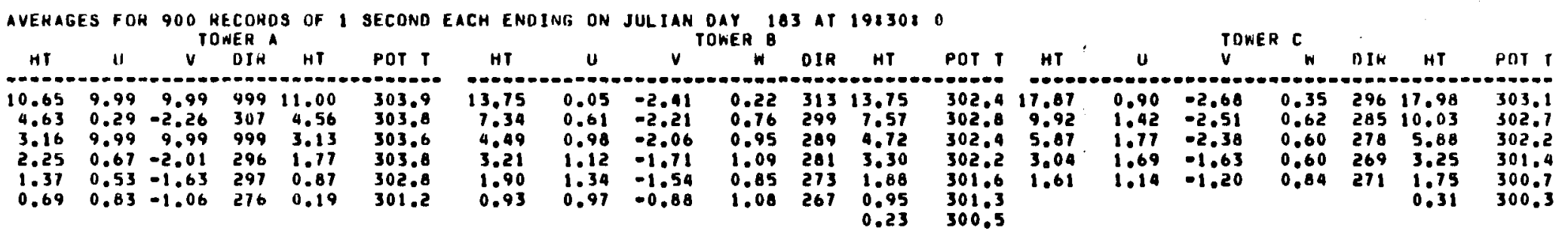

NET RADIATION AT 15M= -54.W/MAL2, AT IME -44,W/MMH2

AVERAGES FOR 900 RECOROS OF I SECOND EACH ENDING ON JULIAN DAY 103 AT 1984510

\begin{tabular}{|c|c|c|c|c|c|c|c|c|c|c|c|c|c|c|c|c|c|c|c|}
\hline $\mathrm{HT}$ & u & $v$ & DIR & HT & POT $T$ & HT & 11 & $v$ & $w$ & DIK & HT & POT T & HT & U & $v$ & & OSR & HT & POT \\
\hline $\begin{array}{r}10.65 \\
4.63 \\
3.16 \\
2.25 \\
1.37 \\
0.69\end{array}$ & $\begin{array}{l}9.99 \\
0.01 \\
9.99 \\
0.47 \\
0.45 \\
0.81\end{array}$ & $\begin{array}{r}9.99 \\
-1.76 \\
9.99 \\
-1.67 \\
-1.41 \\
-0.91\end{array}$ & $\begin{array}{l}9991 \\
314 \\
999 \\
299 \\
297 \\
273\end{array}$ & $\begin{array}{r}11.00 \\
4.56 \\
3.13 \\
1.17 \\
0.87 \\
0.19\end{array}$ & $\begin{array}{l}303.7 \\
303.7 \\
303.6 \\
303.8 \\
302.7 \\
300.6\end{array}$ & $\begin{array}{r}13.75 \\
7.34 \\
4.49 \\
3.21 \\
1.90 \\
0.93\end{array}$ & $\begin{array}{r}-0.24 \\
0.39 \\
0.77 \\
0.98 \\
1.22 \\
1.09\end{array}$ & $\begin{array}{l}-1.80 \\
-1.12 \\
-1.69 \\
-1.50 \\
-1.30 \\
-0.86\end{array}$ & $\begin{array}{l}0.02 \\
0.55 \\
0.79 \\
0.99 \\
1.14 \\
1.13\end{array}$ & $\begin{array}{l}322 \\
302 \\
290 \\
261 \\
273 \\
263\end{array}$ & $\begin{array}{l}13.75 \\
7.57 \\
9.72 \\
3.30 \\
1.88 \\
0.95 \\
0.23\end{array}$ & $\begin{array}{l}302.0 \\
302.8 \\
302.5 \\
302.3 \\
301.6 \\
301.0 \\
300.0\end{array}$ & $\begin{array}{r}17.87 \\
9.92 \\
5.87 \\
3.04 \\
1.61\end{array}$ & $\begin{array}{l}0.61 \\
1.19 \\
1.57 \\
1.84 \\
1.32\end{array}$ & $\begin{array}{l}-2.24 \\
-2.12 \\
-2.11 \\
-1.46 \\
-1.03\end{array}$ & $\begin{array}{l}0.29 \\
0.54 \\
0.53 \\
0.62 \\
0.93\end{array}$ & $\begin{array}{l}299 \\
285 \\
278 \\
263 \\
262\end{array}$ & $\begin{array}{r}17.98 \\
10.03 \\
5.88 \\
3.25 \\
1.75 \\
0.31\end{array}$ & $\begin{array}{l}303.0 \\
302.6 \\
302.3 \\
301.2 \\
299.9 \\
299.1\end{array}$ \\
\hline
\end{tabular}

NET RADIATION AT 15M= $-54, W / M * 221$ AT IMX $-44, W / M M E 2$

AVERAGES FOR 900 RECURDS OF I SECOND EACH ENDING DN JULIAN DAY 103 AT 208 O8 0

\begin{tabular}{|c|c|c|c|c|c|c|c|c|c|c|c|c|c|c|c|c|c|c|c|}
\hline HT & u & $v$ & DIR & HT & POT T & HT & $u$ & $v$ & $N$ & DIR & HT & Pot $t$ & HT & $u$ & $v$ & w & DIR & HT & POT T \\
\hline $\begin{array}{r}10.65 \\
4.63 \\
3.16 \\
2.25 \\
1.37 \\
0.69\end{array}$ & $\begin{array}{l}9.99 \\
0.12 \\
9.99 \\
0.53 \\
0.46 \\
0.82\end{array}$ & $\begin{array}{r}9.99 \\
-2.13 \\
9.99 \\
-1.93 \\
-1.63 \\
-1.08\end{array}$ & $\begin{array}{l}999 \\
311 \\
999 \\
299 \\
299 \\
271\end{array}$ & $\begin{array}{r}11.00 \\
4.56 \\
3.13 \\
1.71 \\
0.87 \\
0.19\end{array}$ & $\begin{array}{l}303.4 \\
303.4 \\
303.2 \\
303.4 \\
302.2 \\
300.2\end{array}$ & $\begin{array}{r}13.75 \\
7.34 \\
4.49 \\
3.21 \\
1.90 \\
0.93\end{array}$ & $\begin{array}{r}-0.14 \\
0.36 \\
0.69 \\
0.83 \\
1.04 \\
0.96\end{array}$ & $\begin{array}{l}-2.45 \\
-2.08 \\
-1.92 \\
-1.68 \\
-1.54 \\
-1.03\end{array}$ & $\begin{array}{r}-0.00 \\
0.54 \\
0.71 \\
0.90 \\
1.05 \\
1.04\end{array}$ & $\begin{array}{l}318 \\
305 \\
295 \\
288 \\
281 \\
271\end{array}$ & $\begin{array}{r}13.75 \\
7.57 \\
4.72 \\
3.30 \\
1.88 \\
0.95 \\
0.23\end{array}$ & $\begin{array}{l}301.7 \\
302.4 \\
302.1 \\
301.9 \\
301.3 \\
300.7 \\
299.8\end{array}$ & $\begin{array}{r}17.87 \\
9.92 \\
5.87 \\
3.04 \\
1.61\end{array}$ & $\begin{array}{l}0.75 \\
1.15 \\
1.46 \\
1.63 \\
1.17\end{array}$ & $\begin{array}{l}-2.72 \\
-2.39 \\
-2.16 \\
-1.60 \\
-1.14\end{array}$ & $\begin{array}{l}0.33 \\
0.51 \\
0.48 \\
0.52 \\
0.05\end{array}$ & $\begin{array}{l}299 \\
289 \\
281 \\
269 \\
269\end{array}$ & $\begin{array}{r}17.98 \\
10.03 \\
5.88 \\
3.25 \\
1.75 \\
0.31\end{array}$ & $\begin{array}{l}302.6 \\
302.2 \\
301.9 \\
300.9 \\
299.6 \\
298.8\end{array}$ \\
\hline
\end{tabular}

NET RADIATION AT 15M= $-54 . W / M A M 2$ I AT IM= $-44 . W / M * * 2$ 
$\underline{\text { JULY }} \underline{2-3}, \underline{1981: 1813-0345} \underline{\text { PST }}$

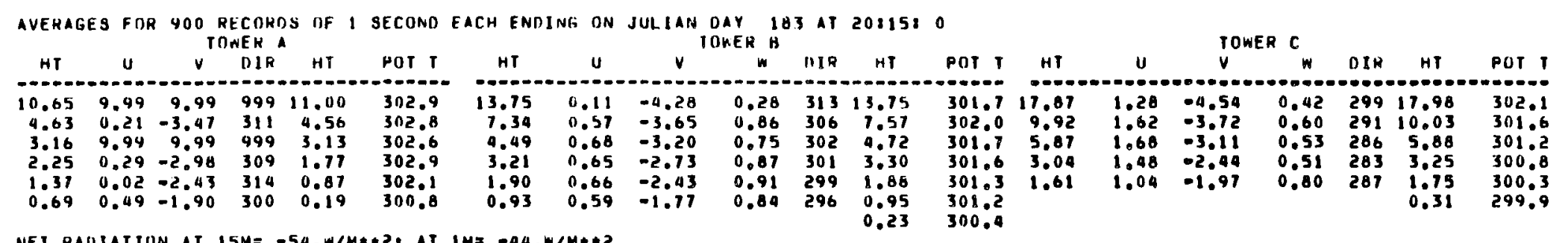

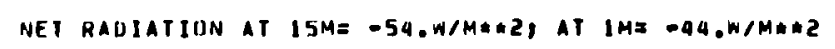

AVEHAGES FOR 900 RECOROS OF 1 SECOND EACH ENOING ON JULIAN DAY 183 AT 2083080

\begin{tabular}{|c|c|c|c|c|c|c|c|c|c|c|c|c|c|c|c|c|c|c|c|}
\hline HT & u & $v$ & OIR & HT & POT T & HT & U & $v$ & $w$ & DIR & HT & POT $T$ & HT & u & $v$ & $w$ & DIR & HT & POT $T$ \\
\hline $\begin{array}{r}10.65 \\
4.63 \\
3.16 \\
2.25 \\
1.37 \\
0.69\end{array}$ & $\begin{array}{l}9.99 \\
0.12 \\
9.99 \\
0.34 \\
0.12 \\
0.54\end{array}$ & $\begin{array}{r}9.99 \\
-3.06 \\
9.99 \\
-2.62 \\
-2.12 \\
-1.60\end{array}$ & $\begin{array}{l}999 \\
312 \\
999 \\
307 \\
311 \\
296\end{array}$ & $\begin{array}{r}11.00 \\
4.56 \\
3.11 \\
1.77 \\
0.87 \\
0.19\end{array}$ & $\begin{array}{l}303.0 \\
302.8 \\
302.6 \\
302.9 \\
302.0 \\
300.6\end{array}$ & $\begin{array}{r}13.75 \\
7.34 \\
4.49 \\
3.21 \\
1.90 \\
0.93\end{array}$ & $\begin{array}{r}-0.03 \\
0.40 \\
0.59 \\
0.60 \\
0.63 \\
0.60\end{array}$ & $\begin{array}{l}-3.53 \\
-3.06 \\
-2.11 \\
-2.30 \\
-2.06 \\
-1.49\end{array}$ & $\begin{array}{l}0.17 \\
0.69 \\
0.69 \\
0.79 \\
0.85 \\
0.81\end{array}$ & $\begin{array}{l}315 \\
307 \\
302 \\
300 \\
298 \\
292\end{array}$ & $\begin{array}{r}13.75 \\
7.51 \\
4.72 \\
3.30 \\
1.88 \\
0.95 \\
0.23\end{array}$ & $\begin{array}{l}301.7 \\
302.1 \\
301.8 \\
301.6 \\
301.3 \\
301.1 \\
300.3\end{array}$ & $\begin{array}{r}17.87 \\
9.92 \\
5.87 \\
3.04 \\
1.61\end{array}$ & $\begin{array}{l}1.02 \\
1.48 \\
1.71 \\
1.38 \\
1.09\end{array}$ & $\begin{array}{l}-3.77 \\
-3.14 \\
-2.75 \\
-2.06 \\
-1.57\end{array}$ & $\begin{array}{l}0.38 \\
0.60 \\
0.55 \\
0.55 \\
0.82\end{array}$ & $\begin{array}{l}299 \\
289 \\
283 \\
271 \\
280\end{array}$ & $\begin{array}{r}17.98 \\
10.03 \\
5.88 \\
3.25 \\
1.75 \\
0.31\end{array}$ & $\begin{array}{l}302.2 \\
301.7 \\
301.2 \\
300.6 \\
299.9 \\
299.5\end{array}$ \\
\hline
\end{tabular}

t

NET RAOIATION AT 15M= -54,W/MMH2, AT 1ME -44,W/M\#HZ

AVERAGES FOH 900 RECUROS OF 1 SECUND EACH ENDING ON JULIAN DAY 183 AI 2084580

\begin{tabular}{|c|c|c|c|c|c|c|c|c|c|c|c|c|c|c|c|c|c|c|c|}
\hline HT & "I & $v$ & DIR & HI & POT 1 & $\mathrm{HT}$ & $u$ & $v$ & $w$ & DIR & HT & POT & HT & U & v & $w$ & DIR & HT & POT $T$ \\
\hline $\begin{array}{r}10.65 \\
4.63 \\
3.16 \\
2.25 \\
1.37 \\
0.69\end{array}$ & $\begin{array}{l}9.99 \\
0.07 \\
9.94 \\
0.32 \\
0.14 \\
0.56\end{array}$ & $\begin{array}{r}9.99 \\
-2.83 \\
9.99 \\
-2.44 \\
-1.96 \\
-1.45\end{array}$ & $\begin{array}{l}999 \\
313 \\
999 \\
307 \\
310 \\
293\end{array}$ & $\begin{array}{r}11.00 \\
4.56 \\
3.13 \\
1.71 \\
0.87 \\
0.19\end{array}$ & $\begin{array}{l}303.2 \\
302.9 \\
302.9 \\
302.9 \\
301.9 \\
300.5\end{array}$ & $\begin{array}{r}13.75 \\
7.34 \\
4.49 \\
3.21 \\
1.90 \\
0.93\end{array}$ & $\begin{array}{r}-0.14 \\
0.37 \\
0.59 \\
0.67 \\
0.75 \\
0.69\end{array}$ & $\begin{array}{l}-3.22 \\
-2.78 \\
-2.50 \\
-2.14 \\
-1.92 \\
-1.37\end{array}$ & $\begin{array}{l}0.11 \\
0.66 \\
0.70 \\
0.82 \\
0.89 \\
0.86\end{array}$ & $\begin{array}{l}317 \\
307 \\
301 \\
297 \\
293 \\
280\end{array}$ & $\begin{array}{r}13.75 \\
7.57 \\
9.72 \\
3.30 \\
1.88 \\
0.95 \\
0.23\end{array}$ & $\begin{array}{l}301.7 \\
302.1 \\
301.8 \\
301.6 \\
301.02 \\
300.9 \\
299.9\end{array}$ & $\begin{array}{r}17.07 \\
9.92 \\
5.87 \\
3.04 \\
1.61\end{array}$ & $\begin{array}{l}0.87 \\
1.39 \\
1.75 \\
1.68 \\
1.18\end{array}$ & $\begin{array}{l}-3.45 \\
-3.00 \\
-2.71 \\
-1.99 \\
-1.50\end{array}$ & $\begin{array}{l}0.37 \\
0.61 \\
0.59 \\
0.58 \\
0.87\end{array}$ & $\begin{array}{l}300 \\
290 \\
282 \\
274 \\
276\end{array}$ & $\begin{array}{r}17.98 \\
10.03 \\
5.88 \\
3.25 \\
1.75 \\
0.31\end{array}$ & $\begin{array}{l}302.2 \\
301 . .8 \\
301.3 \\
300.5 \\
299.6 \\
299.1\end{array}$ \\
\hline
\end{tabular}

NET RADIATIIIN AT 15M= -54.W/MHAR, AT IME -44.W/MAML

aVERAGES FOR 9O0 RECoros OF 1 SECONO EACH ENOING ON JULIAN DAY 103 AT 218080

\begin{tabular}{|c|c|c|c|c|c|c|c|c|c|c|c|c|c|c|c|c|c|c|c|}
\hline AVERAGES & $S F O R$ & 900 & $\begin{array}{l}\text { ECOROS } \\
\text { WER A }\end{array}$ & & SECONO & EACH ENO & ING ON & JUL IAN & $\begin{array}{l}\text { AY } \\
\text { WER B }\end{array}$ & AT & $21: 08$ & 0 & & & & R C & & & \\
\hline HI & u & $v$ & DIR & HT & POI & H T & u & v & $w$ & DIR & HT & Pot 1 & HT & $u$ & v & $w$ & DIR & HT & POT \\
\hline $\begin{array}{r}10.65 \\
4.63 \\
3.16 \\
2.25 \\
1.37 \\
0.69\end{array}$ & $\begin{array}{l}4.99 \\
0.13 \\
9.99 \\
0.43 \\
0.26 \\
0.63\end{array}$ & $\begin{array}{r}9.99 \\
-2.50 \\
9.99 \\
-2.22 \\
-1.81 \\
-1.28\end{array}$ & $\begin{array}{l}9991 \\
312 \\
999 \\
303 \\
306 \\
288\end{array}$ & $\begin{array}{l}11.00 \\
4.56 \\
3.13 \\
1.77 \\
0.87 \\
0.19\end{array}$ & $\begin{array}{l}303.2 \\
303.0 \\
302.8 \\
303.1 \\
302.0 \\
300.4\end{array}$ & $\begin{array}{r}13.75 \\
7.34 \\
4.49 \\
3.21 \\
1.90 \\
0.93\end{array}$ & $\begin{array}{r}-0.18 \\
0.36 \\
0.65 \\
0.78 \\
0.93 \\
0.87\end{array}$ & $\begin{array}{l}-2.76 \\
-2.43 \\
-2.17 \\
-1.86 \\
-1.70 \\
-1.16\end{array}$ & $\begin{array}{l}0.08 \\
0.59 \\
0.73 \\
0.88 \\
1.00 \\
0.97\end{array}$ & $\begin{array}{l}318 \\
306 \\
298 \\
292 \\
286 \\
278\end{array}$ & $\begin{array}{r}13.75 \\
7.57 \\
9.72 \\
3.30 \\
1.88 \\
0.95 \\
0.23\end{array}$ & $\begin{array}{l}301.6 \\
302.2 \\
301.8 \\
301.8 \\
301.0 \\
300.6 \\
299.5\end{array}$ & $\begin{array}{r}17.87 \\
9.92 \\
5.87 \\
3.04 \\
1.61\end{array}$ & $\begin{array}{l}0.71 \\
1.25 \\
1.60 \\
1.65 \\
1.18\end{array}$ & $\begin{array}{l}-2.91 \\
-2.56 \\
-2.41 \\
-1.78 \\
-1.32\end{array}$ & $\begin{array}{l}0.33 \\
0.56 \\
0.54 \\
0.56 \\
0.86\end{array}$ & $\begin{array}{l}301 \\
288 \\
281 \\
272 \\
273\end{array}$ & $\begin{array}{r}17.98 \\
10.03 \\
5.88 \\
3.25 \\
1.75 \\
0.31\end{array}$ & $\begin{array}{l}302.3 \\
301.9 \\
301.5 \\
300.6 \\
299.5 \\
298.8\end{array}$ \\
\hline
\end{tabular}

NET RAOIATIUN AT $154=-54$,W/M*\#2, AT $1 M=-44$, W/M**2 
JULY 2-3, 1981: 1813-0345 PST

\begin{tabular}{|c|c|c|c|c|c|c|c|c|c|c|c|c|c|c|c|c|c|c|c|}
\hline AVERAGE & ES FOK & $900 \mathrm{TO}$ & $\begin{array}{l}\text { ECORO } \\
\text { NER A }\end{array}$ & & SECOND & EACH & ING ON & JUL IAN & $\begin{array}{l}\text { DAY } \\
\text { rOWER B }\end{array}$ & & 111 & & & & & 0 & & & \\
\hline HT & U & $v$ & DIR & HT & POT T & HT & $\mathbf{u}$ & $v$ & $w$ & DIR & HT & POT T & HT & & & & DIR & HT & POT \\
\hline $\begin{array}{r}10.65 \\
4.63 \\
3.16 \\
2.25 \\
1.37 \\
0.69\end{array}$ & $\begin{array}{r}9.99 \\
-0.12 \\
9.99 \\
0.20 \\
0.14 \\
0.52\end{array}$ & $\begin{array}{r}9.99 \\
-2.02 \\
9.99 \\
-1.84 \\
-1.48 \\
-1.08\end{array}$ & $\begin{array}{l}999 \\
318 \\
994 \\
308 \\
309 \\
289\end{array}$ & $\begin{array}{r}11.00 \\
4.56 \\
3.13 \\
1.71 \\
0.87 \\
0.19\end{array}$ & $\begin{array}{l}303.0 \\
303.0 \\
302.8 \\
303.1 \\
302.0 \\
300.2\end{array}$ & $\begin{array}{r}13.75 \\
7.34 \\
4.49 \\
3.21 \\
1.90 \\
0.93\end{array}$ & $\begin{array}{r}-0.24 \\
0.22 \\
0.50 \\
0.64 \\
0.82 \\
0.83\end{array}$ & $\begin{array}{l}-2.25 \\
-1.99 \\
-1.83 \\
-1.61 \\
-1.50 \\
-1.03\end{array}$ & $\begin{array}{r}-0.00 \\
0.47 \\
0.59 \\
0.75 \\
0.89 \\
0.91\end{array}$ & $\begin{array}{l}321 \\
30 A \\
299 \\
293 \\
286 \\
276\end{array}$ & $\begin{array}{r}13.15 \\
7.57 \\
4.72 \\
3.30 \\
1.88 \\
0.95 \\
0.23\end{array}$ & $\begin{array}{l}301.4 \\
302.1 \\
301.9 \\
301.7 \\
301.1 \\
300.7 \\
299.5\end{array}$ & $\begin{array}{r}17.87 \\
9.92 \\
5.87 \\
3.04 \\
1.61\end{array}$ & $\begin{array}{l}0.63 \\
1.07 \\
1.39 \\
1.64 \\
1.26\end{array}$ & $\begin{array}{l}-2.32 \\
-2.09 \\
-2.02 \\
-1.55 \\
-1.10\end{array}$ & $\begin{array}{l}0.30 \\
0.49 \\
0.45 \\
0.54 \\
0.89\end{array}$ & $\begin{array}{l}299 \\
287 \\
280 \\
268 \\
266\end{array}$ & $\begin{array}{r}17.98 \\
10.03 \\
5.88 \\
3.25 \\
1.75 \\
0.31\end{array}$ & $\begin{array}{l}302.1 \\
301.9 \\
301.6 \\
300.8 \\
299.3 \\
298.1\end{array}$ \\
\hline
\end{tabular}

NET RADIATION AT 15M= -54.W/MMAL, AT IME -44.W/MA\#2

AVERAGES FOR 900 RECOHOS OF 1 SECOND EACH ENOING ON JULIAN DAY 103 AT 2113010

\begin{tabular}{|c|c|c|c|c|c|c|c|c|c|c|c|c|c|c|c|c|c|c|c|}
\hline HT & $u$ & $v$ & DIR & HT & POI 1 & HT & $u$ & $v$ & $\omega$ & OIR & HT & POT & HT & u & $v$ & $w$ & DIR & HT & POT \\
\hline $\begin{array}{l}10.65 \\
4.63 \\
3.16 \\
2.25 \\
1.37 \\
0.69\end{array}$ & $\begin{array}{l}9.99 \\
0.23 \\
9.99 \\
0.12 \\
0.09 \\
0.50\end{array}$ & $\begin{array}{r}9.99 \\
-2.02 \\
9.99 \\
-1.90 \\
-1.56 \\
-1.11\end{array}$ & $\begin{array}{l}999 \\
321 \\
999 \\
311 \\
311 \\
291\end{array}$ & $\begin{array}{r}11.00 \\
4.56 \\
3.13 \\
1.77 \\
0.87 \\
0.19\end{array}$ & $\begin{array}{l}303.0 \\
303.0 \\
302.8 \\
303.1 \\
302.0 \\
300.3\end{array}$ & $\begin{array}{r}13.75 \\
7.34 \\
9.49 \\
3.21 \\
1.90 \\
0.93\end{array}$ & $\begin{array}{r}-0.21 \\
0.20 \\
0.44 \\
0.56 \\
0.72 \\
0.75\end{array}$ & $\begin{array}{l}-2.23 \\
-1.90 \\
-1.87 \\
-1.66 \\
-1.53 \\
-1.09\end{array}$ & $\begin{array}{l}0.02 \\
0.44 \\
0.55 \\
0.70 \\
0.82 \\
0.86\end{array}$ & $\begin{array}{l}320 \\
309 \\
301 \\
296 \\
289 \\
280\end{array}$ & $\begin{array}{r}13.75 \\
7.57 \\
9.72 \\
3.30 \\
1.88 \\
0.95 \\
0.23\end{array}$ & $\begin{array}{l}301.4 \\
302.02 \\
302.0 \\
301.8 \\
301.3 \\
300.9 \\
299.9\end{array}$ & $\begin{array}{r}17.87 \\
9.92 \\
9.87 \\
3.04 \\
1.61\end{array}$ & $\begin{array}{l}0.67 \\
1.11 \\
1.40 \\
1.63 \\
1.21\end{array}$ & $\begin{array}{l}-2.41 \\
-2.16 \\
-2.08 \\
-1.65 \\
-1.18\end{array}$ & $\begin{array}{l}0.30 \\
0.50 \\
0.46 \\
0.54 \\
0.87\end{array}$ & $\begin{array}{l}299 \\
207 \\
281 \\
270 \\
269\end{array}$ & $\begin{array}{r}17.98 \\
10.03 \\
5.88 \\
3.25 \\
1.75 \\
0.31\end{array}$ & $\begin{array}{l}302.2 \\
301.9 \\
301.7 \\
301.0 \\
299.6 \\
298.5\end{array}$ \\
\hline
\end{tabular}

AVERAGES FOR 900 RECOROS OF 1 SECOND EACH ENOING ON JULIAN DAY 103 AT 2114580

\begin{tabular}{|c|c|c|c|c|c|c|c|c|c|c|c|c|c|c|c|c|c|c|c|}
\hline HT & u & $v$ & DIH & HT & ז' ז' & $\begin{array}{r}n T \\
-n\end{array}$ & u & $v$ & w & DIR & HT & POT T & HT & $u$ & $v$ & $=$ & DIR & HT & POI T \\
\hline $\begin{array}{r}10.65 \\
4.63 \\
3.16 \\
2.25 \\
1.37 \\
0.69\end{array}$ & $\begin{array}{l}9.99 \\
0.110 \\
9.99 \\
0.47 \\
0.37 \\
0.72\end{array}$ & $\begin{array}{r}9.99 \\
-2.06 \\
9.99 \\
-1.91 \\
-1.55 \\
-1.06\end{array}$ & $\begin{array}{l}999 \\
312 \\
999 \\
301 \\
301 \\
200\end{array}$ & $\begin{array}{r}11.00 \\
4.56 \\
3.13 \\
1.77 \\
0.87 \\
0.19\end{array}$ & $\begin{array}{l}303.2 \\
303.2 \\
303.0 \\
303.2 \\
302.1 \\
300.2\end{array}$ & $\begin{array}{r}13.75 \\
7.34 \\
4.49 \\
3.21 \\
1.90 \\
0.93\end{array}$ & $\begin{array}{l}0.07 \\
0.09 \\
0.70 \\
0.90 \\
1.09 \\
1.00\end{array}$ & $\begin{array}{l}-2.17 \\
-1.98 \\
-1.80 \\
-1.64 \\
-1.50 \\
-1.00\end{array}$ & $\begin{array}{l}0.25 \\
0.68 \\
0.79 \\
0.95 \\
1.08 \\
1.06\end{array}$ & $\begin{array}{l}313 \\
301 \\
293 \\
286 \\
270 \\
270\end{array}$ & $\begin{array}{l}13.75 \\
7.57 \\
4.72 \\
3.30 \\
1.88 \\
0.95 \\
0.23\end{array}$ & $\begin{array}{l}301,5 \\
302,3 \\
302,0 \\
301.8 \\
301.2 \\
300.7 \\
299,5\end{array}$ & $\begin{array}{r}17.87 \\
9.92 \\
5.67 \\
3.04 \\
1.61\end{array}$ & $\begin{array}{l}0.98 \\
1.32 \\
1.58 \\
1.75 \\
1.29\end{array}$ & $\begin{array}{l}-2.20 \\
-2.00 \\
-2.01 \\
-1.50 \\
-1.07\end{array}$ & $\begin{array}{l}0.35 \\
0.55 \\
0.52 \\
0.59 \\
0.90\end{array}$ & $\begin{array}{l}291 \\
282 \\
276 \\
265 \\
264\end{array}$ & $\begin{array}{r}17.90 \\
10.03 \\
5.00 \\
3.25 \\
1.75 \\
0.31\end{array}$ & $\begin{array}{l}302.3 \\
302.1 \\
301.9 \\
300.9 \\
299.4 \\
298.4\end{array}$ \\
\hline
\end{tabular}

NET RADIATION AT ISM= -54,W/MEMLI AT IME -44,W/MAML

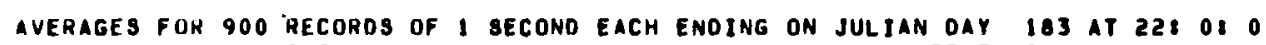

\begin{tabular}{|c|c|c|c|c|c|c|c|c|c|c|c|c|c|c|c|c|c|c|c|}
\hline HI & u & $v$ & DIR & $\mathrm{HT}$ & POI & HT & u & $v$ & $w$ & DIR & HT & Por T & HT & U & $v$ & $w$ & OIR & HT & POT \\
\hline $\begin{array}{r}10.65 \\
4.63 \\
3.16 \\
2.25 \\
1.37 \\
0.69\end{array}$ & $\begin{array}{l}9.99 \\
0.12 \\
9.99 \\
0.51 \\
0.38 \\
0.73\end{array}$ & $\begin{array}{r}9.99 \\
-2.222 \\
9.99 \\
-2.01 \\
-1.64 \\
-1.110\end{array}$ & $\begin{array}{l}999 \\
311 \\
999 \\
300 \\
301 \\
281\end{array}$ & $\begin{array}{r}11.00 \\
4.56 \\
3.13 \\
1.77 \\
0.81 \\
0.19\end{array}$ & $\begin{array}{l}303.1 \\
303.1 \\
302.8 \\
303.0 \\
301.9 \\
300.2\end{array}$ & $\begin{array}{r}13.75 \\
7.34 \\
4.49 \\
3.21 \\
1.90 \\
0.93\end{array}$ & $\begin{array}{r}-0.14 \\
0.37 \\
0.73 \\
0.92 \\
1.11 \\
0.99\end{array}$ & $\begin{array}{l}-2.33 \\
-2.08 \\
-1.95 \\
-1.71 \\
-1.55 \\
-1.03\end{array}$ & $\begin{array}{l}0.09 \\
0.59 \\
0.76 \\
0.95 \\
1.10 \\
1.07\end{array}$ & $\begin{array}{l}318 \\
304 \\
294 \\
286 \\
279 \\
270\end{array}$ & $\begin{array}{r}13.75 \\
7.57 \\
4.72 \\
3.30 \\
1.80 \\
0.95 \\
0.23\end{array}$ & $\begin{array}{l}301.4 \\
302.2 \\
301.8 \\
301.5 \\
300.9 \\
300.4 \\
299.5\end{array}$ & $\begin{array}{r}17.87 \\
9.92 \\
3.87 \\
3.04 \\
1.61\end{array}$ & $\begin{array}{l}0.64 \\
1.16 \\
1.55 \\
1.84 \\
1.32\end{array}$ & $\begin{array}{l}-2.40 \\
-2.22 \\
-2.19 \\
-1.61 \\
-1.10\end{array}$ & $\begin{array}{l}0.30 \\
0.52 \\
0.51 \\
0.60 \\
0.93\end{array}$ & $\begin{array}{l}299 \\
287 \\
279 \\
266 \\
264\end{array}$ & $\begin{array}{r}17.98 \\
10.03 \\
5.88 \\
3.25 \\
1.75 \\
0.31\end{array}$ & $\begin{array}{l}302.3 \\
301.9 \\
301.6 \\
300.6 \\
299.0 \\
298.0\end{array}$ \\
\hline
\end{tabular}


JULY 2-3. 1981: 1813-0345 PST

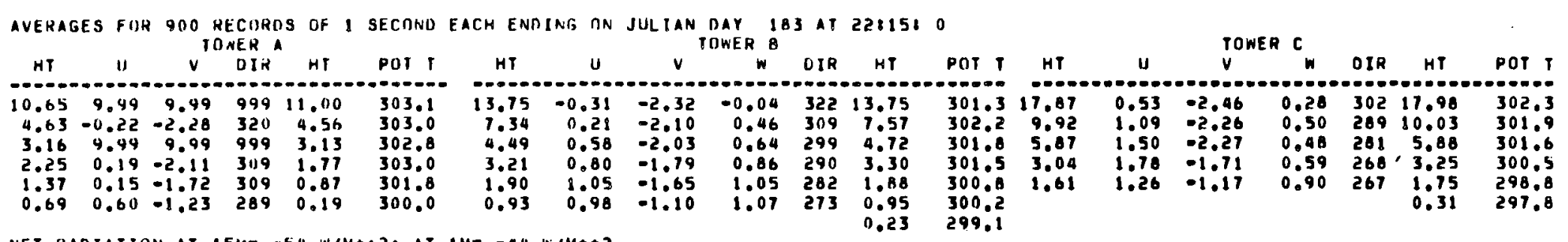

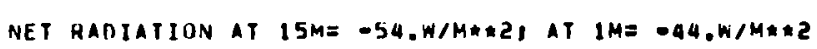

AVERAGES FOR 9OO RECORDS OF I SECOND EACH ENDING ON JULIAN DAY 183 AT 2283010

\begin{tabular}{|c|c|c|c|c|c|c|c|c|c|c|c|c|c|c|c|c|c|c|c|}
\hline HT & 11 & $v$ & OIR & HI & POT T & HT & u & $v$ & $w$ & DIR & NT & POT T & KT & u & $v^{\text {rom }}$ & & DIR & HT & POT \\
\hline $\begin{array}{r}10.65 \\
4.63 \\
3.16 \\
2.25 \\
1.37 \\
0.69\end{array}$ & $\begin{array}{r}9.99 \\
-0.43 \\
9.99 \\
-0.05 \\
-0.07 \\
0.39\end{array}$ & $\begin{array}{r}9.99 \\
-2.45 \\
9.99 \\
-2.18 \\
-1.76 \\
-1.36\end{array}$ & $\begin{array}{l}999 \\
324 \\
999 \\
316 \\
317 \\
299\end{array}$ & $\begin{array}{r}11.00 \\
4.56 \\
3.13 \\
1.717 \\
0.87 \\
0.19\end{array}$ & $\begin{array}{l}302.8 \\
302.7 \\
302.4 \\
302.6 \\
301.5 \\
299.9\end{array}$ & $\begin{array}{r}13.75 \\
7.34 \\
4.49 \\
3.21 \\
1.90 \\
0.93\end{array}$ & $\begin{array}{r}-0.52 \\
-0.08 \\
0.26 \\
0.42 \\
0.60 \\
0.64\end{array}$ & $\begin{array}{l}-2.73 \\
-2.46 \\
-2.22 \\
-1.90 \\
-1.72 \\
-1.23\end{array}$ & $\begin{array}{l}-0.27 \\
0.21 \\
0.36 \\
0.61 \\
0.74 \\
0.79\end{array}$ & $\begin{array}{l}325 \\
316 \\
308 \\
302 \\
295 \\
207\end{array}$ & $\begin{array}{r}13.75 \\
7.57 \\
4.72 \\
3.30 \\
1.88 \\
0.95 \\
0.23\end{array}$ & $\begin{array}{l}301.2 \\
301.9 \\
301.8 \\
301.4 \\
300.8 \\
300.4 \\
299.2\end{array}$ & $\begin{array}{r}17.87 \\
9.92 \\
5.87 \\
3.09 \\
1.61\end{array}$ & $\begin{array}{l}0.29 \\
0.85 \\
1.36 \\
1.63 \\
1.17\end{array}$ & $\begin{array}{l}-2.07 \\
-2.63 \\
-2.43 \\
-1.95 \\
-1.39\end{array}$ & $\begin{array}{l}0.28 \\
0.44 \\
0.43 \\
0.56 \\
0.88\end{array}$ & $\begin{array}{l}310 \\
297 \\
285 \\
275 \\
274\end{array}$ & $\begin{array}{r}17.98 \\
10.03 \\
5.80 \\
3.25 \\
8.75 \\
0.31\end{array}$ & $\begin{array}{l}302.1 \\
301.7 \\
301.3 \\
300.9 \\
298.9 \\
297.9\end{array}$ \\
\hline
\end{tabular}

N

\begin{tabular}{|c|c|c|c|c|c|c|c|c|c|c|c|c|c|c|c|c|c|c|c|}
\hline VEHAGES & $\begin{array}{c}S \text { FOR } \\
\text { U }\end{array}$ & $v^{00}$ & $\begin{array}{l}\text { ECOROS } \\
\text { NER A } \\
\text { DIR }\end{array}$ & 3 of 1 & SECOND & EACH ENDINC & IG ON & JULI IAN & $\begin{array}{l}\text { ir } \\
\text { IER } \\
\text { W }\end{array}$ & $\begin{array}{l}3 \text { AT } \\
\text { OIA }\end{array}$ & $\begin{array}{c}228451 \\
\text { HT }\end{array}$ & POT & HT & $\mathbf{u}$ & $v^{\text {TOWER }}$ & & DIR & NT & P01 \\
\hline $\begin{array}{r}10.65 \\
4.63 \\
3.16 \\
2.25 \\
1.37 \\
0.69\end{array}$ & $\begin{array}{l}9.99 \\
0.08 \\
9.99 \\
0.39 \\
0.25 \\
0.65\end{array}$ & $\begin{array}{r}9.99 \\
-2.63 \\
9.99 \\
-2.34 \\
-1.90 \\
-1.38\end{array}$ & $\begin{array}{l}999 \\
313 \\
999 \\
305 \\
307 \\
289\end{array}$ & $\begin{array}{r}11.00 \\
4.56 \\
3.13 \\
1.77 \\
0.87 \\
0.19\end{array}$ & $\begin{array}{l}302.8 \\
302.6 \\
302.3 \\
302.5 \\
301.04 \\
299.7\end{array}$ & $\begin{array}{r}13.75 \\
7.34 \\
4.49 \\
3.21 \\
1.90 \\
0.93\end{array}$ & $\begin{array}{l}0.03 \\
0.49 \\
0.00 \\
0.95 \\
1.12 \\
0.96\end{array}$ & $\begin{array}{l}-2.67 \\
-2.43 \\
-2.310 \\
-1.97 \\
-1.78 \\
-1.21\end{array}$ & $\begin{array}{l}0.23 \\
0.70 \\
0.83 \\
1.08 \\
1.14 \\
1.09\end{array}$ & $\begin{array}{l}314 \\
303 \\
295 \\
289 \\
282 \\
275\end{array}$ & $\begin{array}{l}13.75 \\
1.57 \\
4.72 \\
3.30 \\
1.88 \\
0.95 \\
0.23\end{array}$ & $\begin{array}{l}301.1 \\
301.8 \\
301.8 \\
301.1 \\
300.3 \\
299.9 \\
298.7\end{array}$ & $\begin{array}{r}17.87 \\
9.92 \\
5.87 \\
3.04 \\
1.61\end{array}$ & $\begin{array}{l}0.80 \\
1.38 \\
1.77 \\
1.92 \\
1.31\end{array}$ & $\begin{array}{l}-2.80 \\
-2.59 \\
-2.57 \\
-1.86 \\
-1.33\end{array}$ & $\begin{array}{l}0.36 \\
0.60 \\
0.60 \\
0.65 \\
0.94\end{array}$ & $\begin{array}{l}297 \\
287 \\
280 \\
269 \\
270\end{array}$ & $\begin{array}{r}17.98 \\
10.03 \\
5.88 \\
3.25 \\
1.75 \\
0.31\end{array}$ & $\begin{array}{l}301.9 \\
301.6 \\
301.2 \\
300.0 \\
298.7 \\
297.9\end{array}$ \\
\hline
\end{tabular}

NET RADIATION AT 15ME -54.W/MA\#2, AT IME -44.W/MHAZ

AVERAGES FOR 900 RECOHDS OF I SECOND EACH ENDING ON JULIAN DAY 103 AT 231 OI 0

\begin{tabular}{|c|c|c|c|c|c|c|c|c|c|c|c|c|c|c|c|c|c|c|c|}
\hline ח & u & $v$ & DIR & HT & POT & HT & u & $v$ & $w$ & DIR & HT & Pot & & $u$ & $v$ & $w$ & DIR & HT & POT 1 \\
\hline $\begin{array}{l}10.63 \\
4.63 \\
3.16 \\
2.25 \\
1.37 \\
0.69\end{array}$ & $\begin{array}{r}9.99 \\
-0.29 \\
9.99 \\
0.09 \\
0.04 \\
0.49\end{array}$ & $\begin{array}{r}9.99 \\
-2.42 \\
9.99 \\
-2.16 \\
-1.72 \\
-1.30\end{array}$ & $\begin{array}{l}999 \\
321 \\
999 \\
312 \\
313 \\
294\end{array}$ & $\begin{array}{r}11.00 \\
4.56 \\
3.13 \\
1.77 \\
0.87 \\
0.19\end{array}$ & $\begin{array}{l}302.7 \\
302.6 \\
302.3 \\
302.6 \\
301.4 \\
299.7\end{array}$ & $\begin{array}{r}13.75 \\
7.34 \\
4.49 \\
3.21 \\
1.90 \\
0.93\end{array}$ & $\begin{array}{r}-0.32 \\
0.15 \\
0.45 \\
0.60 \\
0.76 \\
0.76\end{array}$ & $\begin{array}{l}-2.65 \\
-2.31 \\
-2.13 \\
-1.83 \\
-1.67 \\
-1.18\end{array}$ & $\begin{array}{r}-0.12 \\
0.40 \\
0.52 \\
0.72 \\
0.85 \\
0.89\end{array}$ & $\begin{array}{l}321 \\
311 \\
303 \\
296 \\
290 \\
282\end{array}$ & $\begin{array}{r}13.75 \\
7.57 \\
4.72 \\
3.30 \\
1.88 \\
0.95 \\
0.23\end{array}$ & $\begin{array}{l}301.0 \\
301.7 \\
301.4 \\
301.2 \\
300.5 \\
300.1 \\
298.9\end{array}$ & $\begin{array}{l}87.87 \\
9.92 \\
5.87 \\
3.04 \\
1.61\end{array}$ & $\begin{array}{l}0.60 \\
1.14 \\
1.54 \\
1.74 \\
1.23\end{array}$ & $\begin{array}{l}-2.67 \\
-2.44 \\
-2.37 \\
-1.77 \\
-1.21\end{array}$ & $\begin{array}{l}0.30 \\
0.52 \\
0.50 \\
0.59 \\
0.90\end{array}$ & $\begin{array}{l}302 \\
289 \\
281 \\
270 \\
269\end{array}$ & $\begin{array}{r}17.98 \\
10.03 \\
5.88 \\
3.25 \\
8.75 \\
0.31\end{array}$ & $\begin{array}{l}301.8 \\
301.5 \\
301.1 \\
300.0 \\
298.5 \\
297.4\end{array}$ \\
\hline
\end{tabular}

NET RADIATION AT $15 M=-54, W / M * 2$, AT $1 M=-44 . W / M \# \# 2$ 
JULY $\underline{2-3}, \underline{1981 ;} 1813-0345$ PST

AVERAGES FGH 900 RECOROS OF I SECOND EACH ENOING ON JULIAN DAY 183 AT 2311580

\begin{tabular}{|c|c|c|c|c|c|c|c|c|c|c|c|c|c|c|c|c|c|c|c|}
\hline HT & 13 & $v$ & DIR & HT & POT & HT & v & $v$ & $w$ & DIR & HT & POT T & HT & u & $v$ & $\omega$ & DIA & $\mathrm{HT}$ & TOT \\
\hline $\begin{array}{r}10.65 \\
4.63 \\
3.16 \\
2.25 \\
1.37 \\
0.69\end{array}$ & $\begin{array}{l}9.94 \\
0.26 \\
9.99 \\
0.07 \\
0.04 \\
0.04\end{array}$ & $\begin{array}{r}9.99 \\
-2.50 \\
9.99 \\
-2.22 \\
-1.76 \\
-1.32\end{array}$ & $\begin{array}{l}999 \\
320 \\
999 \\
313 \\
313 \\
295\end{array}$ & $\begin{array}{r}11.00 \\
4.56 \\
3.13 \\
1.17 \\
0.87 \\
0.19\end{array}$ & $\begin{array}{l}302.4 \\
302.3 \\
302.1 \\
302.3 \\
301.1 \\
299.5\end{array}$ & $\begin{array}{r}13.75 \\
7.34 \\
9.49 \\
3.21 \\
1.90 \\
0.93\end{array}$ & $\begin{array}{l}-0.35 \\
0.18 \\
0.44 \\
0.56 \\
0.73 \\
0.74\end{array}$ & $\begin{array}{l}-2.66 \\
-2.33 \\
-2.15 \\
-1.83 \\
-1.69 \\
-1.19\end{array}$ & $\begin{array}{r}-0.11 \\
0.44 \\
0.56 \\
0.71 \\
0.83 \\
0.87\end{array}$ & $\begin{array}{l}322 \\
310 \\
303 \\
297 \\
291 \\
283\end{array}$ & $\begin{array}{l}13.75 \\
7.57 \\
4.72 \\
3.30 \\
1.88 \\
0.95 \\
0.23\end{array}$ & $\begin{array}{l}300.7 \\
301.5 \\
301.2 \\
301.0 \\
300.9 \\
300.0 \\
298.7\end{array}$ & $\begin{array}{r}17.67 \\
9.92 \\
5.87 \\
3.04 \\
1.61\end{array}$ & $\begin{array}{l}0.52 \\
1.10 \\
1.48 \\
1.75 \\
1.27\end{array}$ & $\begin{array}{l}-2.80 \\
-2.43 \\
-2.31 \\
-1.77 \\
-1.22\end{array}$ & $\begin{array}{l}0.30 \\
0.50 \\
0.48 \\
0.59 \\
0.91\end{array}$ & $\begin{array}{l}304 \\
290 \\
282 \\
270 \\
268\end{array}$ & $\begin{array}{r}17.98 \\
10.03 \\
5.88 \\
3.25 \\
1.75 \\
0.31\end{array}$ & $\begin{array}{l}301.6 \\
301.2 \\
300.9 \\
299.8 \\
298.3 \\
297.2\end{array}$ \\
\hline
\end{tabular}

NET RAOIATION AT 154=-54,W/ME*2, AT IME -44.W/M*A2

AVERAGES FOR 9OO RECORDS OF I SECOND EACH ENDING ON JULIAN DAY 103 AT 2313080

\begin{tabular}{|c|c|c|c|c|c|c|c|c|c|c|c|c|c|c|c|c|c|c|c|}
\hline HT & u & $v$ & DIH & HT & POT T & HT & $\mathbf{u}$ & $v$ & $w$ & DIR & HT & POT & $\mathrm{HT}$ & $\mathbf{u}$ & $v$ & & oIn & $\mathrm{HT}$ & POT T \\
\hline $\begin{array}{r}10.65 \\
4.63 \\
3.16 \\
2.25 \\
1.37 \\
0.69\end{array}$ & $\begin{array}{r}9.99 \\
-0.24 \\
9.99 \\
0.12 \\
0.10 \\
0.51\end{array}$ & $\begin{array}{r}9.99 \\
-2.15 \\
9.99 \\
-1.93 \\
-1.57 \\
-1.16\end{array}$ & $\begin{array}{l}999 \\
321 \\
999 \\
311 \\
511 \\
291\end{array}$ & $\begin{array}{r}11.00 \\
4.56 \\
3.13 \\
1.77 \\
0.87 \\
0.19\end{array}$ & $\begin{array}{l}302.3 \\
302.2 \\
302.0 \\
302.2 \\
301.0 \\
299.3\end{array}$ & $\begin{array}{l}13.75 \\
7.34 \\
4.49 \\
3.21 \\
1.90 \\
0.93\end{array}$ & $\begin{array}{r}-0.26 \\
0.20 \\
0.51 \\
0.65 \\
0.88 \\
0.84\end{array}$ & $\begin{array}{l}-2.17 \\
-1.96 \\
-1.80 \\
-1.57 \\
-1 . .47 \\
-1.000\end{array}$ & $\begin{array}{r}-0.00 \\
0.42 \\
0.57 \\
0.73 \\
0.85 \\
0.92\end{array}$ & $\begin{array}{l}321 \\
309 \\
299 \\
292 \\
284 \\
275\end{array}$ & $\begin{array}{r}13.75 \\
7.57 \\
4.72 \\
3.30 \\
1.88 \\
0.95 \\
0.23\end{array}$ & $\begin{array}{l}300.6 \\
301.4 \\
301.1 \\
300.9 \\
300.0 \\
299.8 \\
298.5\end{array}$ & $\begin{array}{r}17.87 \\
9.92 \\
5.87 \\
3.04 \\
1.61\end{array}$ & $\begin{array}{l}0.60 \\
1.08 \\
1.40 \\
1.78 \\
1.31\end{array}$ & $\begin{array}{l}-2.21 \\
-2.01 \\
-1.98 \\
-1.48 \\
-0.91\end{array}$ & $\begin{array}{l}0.29 \\
0.48 \\
0.44 \\
0.56 \\
0.91\end{array}$ & $\begin{array}{l}299 \\
286 \\
279 \\
265 \\
261\end{array}$ & $\begin{array}{r}17.98 \\
10.03 \\
5.80 \\
3.25 \\
1.75 \\
0.31\end{array}$ & $\begin{array}{l}301.4 \\
301.4 \\
300.8 \\
299.9 \\
298.0 \\
296.7\end{array}$ \\
\hline
\end{tabular}

AVERAGES FOR 900 RECORDS OF I SECOND EACH ENDING ON JULIAN DAY 103 AT 2314580

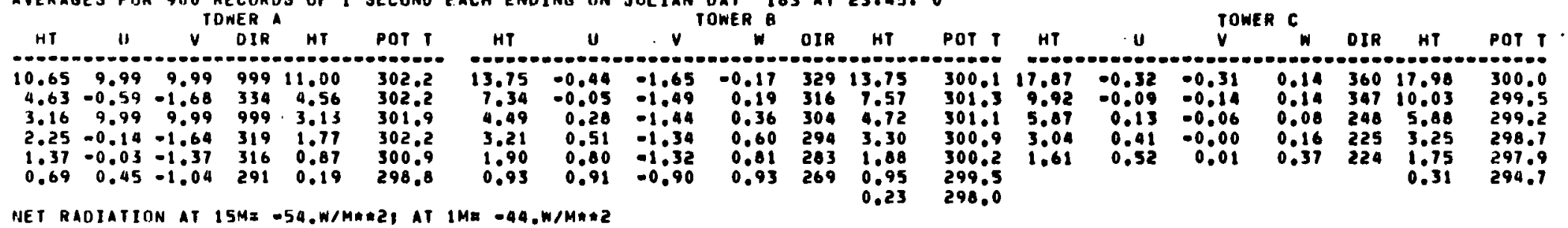

NET RAOIATION AT 15ME -54.W/MMARI AT IMA -44,W/MAMZ

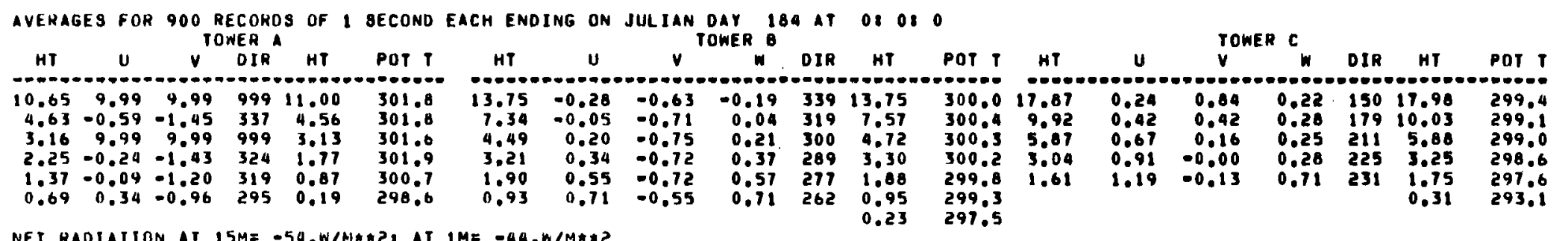

NET RADIATION AT I5M= -54.W/MI\#2, AT IME -44.W/M**2 
$\underline{\text { JULYY }} \underline{2-3}, \underline{1981} ; \underline{1813-0345} \underline{\text { PST }}$

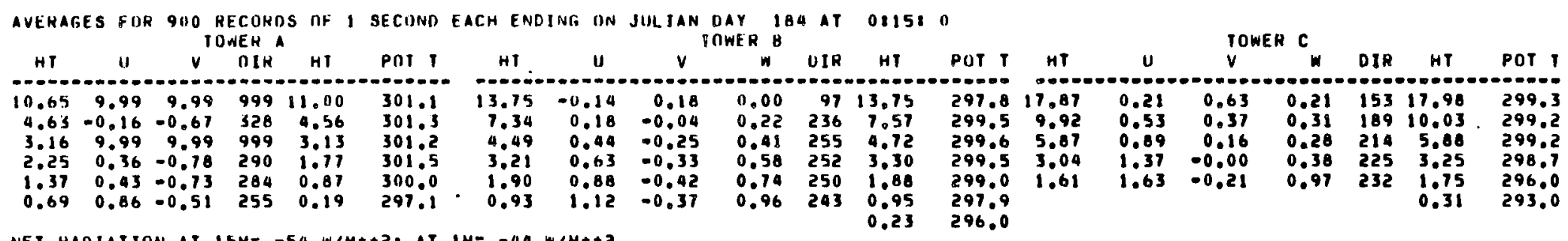

NET HADIATION AT ISM= -54,W/M**2, AT IME -44,W/M\#*2

AVERAGES FOR 900 HECOROS OF I SECOND EACH ENDING ON JULIAN DAY 104 AT 023080

\begin{tabular}{|c|c|c|c|c|c|c|c|c|c|c|c|c|c|c|c|c|c|c|c|}
\hline HI & u & $v$ & OIR & HI & POI T & HT & u & $v$ & $w$ & DIR & HT & POT I & HT & U & $v$ & $w$ & DIA & HT & POT $T$ \\
\hline $\begin{array}{r}10.65 \\
4.63 \\
3.16 \\
2.25 \\
1.37 \\
0.69\end{array}$ & $\begin{array}{l}9.99 \\
0.02 \\
9.99 \\
0.58 \\
0.65 \\
1.04\end{array}$ & $\begin{array}{r}9.99 \\
-0.41 \\
9.99 \\
-0.22 \\
-0.44 \\
-0.21\end{array}$ & $\begin{array}{l}999 \\
312 \\
999 \\
245 \\
259 \\
239\end{array}$ & $\begin{array}{r}11.00 \\
4.56 \\
3.13 \\
1.17 \\
0.87 \\
0.19\end{array}$ & $\begin{array}{l}300.9 \\
301.1 \\
301.0 \\
301.4 \\
299.9 \\
295.5\end{array}$ & $\begin{array}{r}13.75 \\
7.34 \\
4.49 \\
3.21 \\
1.90 \\
0.93\end{array}$ & $\begin{array}{l}0.32 \\
0.51 \\
0.74 \\
0.92 \\
1.21 \\
1.42\end{array}$ & $\begin{array}{r}0.19 \\
0.13 \\
-0.04 \\
-0.08 \\
-0.27 \\
-0.35\end{array}$ & $\begin{array}{l}0.37 \\
0.51 \\
0.68 \\
0.80 \\
0.97 \\
1.22\end{array}$ & $\begin{array}{l}194 \\
210 \\
228 \\
230 \\
237 \\
238\end{array}$ & $\begin{array}{l}13.75 \\
7.57 \\
4.72 \\
3.30 \\
1.88 \\
0.95 \\
0.23\end{array}$ & $\begin{array}{l}297.2 \\
299.5 \\
299.6 \\
299.5 \\
299.0 \\
297.0 \\
295.2\end{array}$ & $\begin{array}{r}17.87 \\
9.92 \\
5.07 \\
3.04 \\
1.61\end{array}$ & $\begin{array}{l}0.90 \\
1.36 \\
1.81 \\
2.39 \\
1.86\end{array}$ & $\begin{array}{r}0.01 \\
-0.07 \\
-0.12 \\
-0.25 \\
-0.36\end{array}$ & $\begin{array}{l}0.32 \\
0.51 \\
0.51 \\
0.67 \\
1.17\end{array}$ & $\begin{array}{l}224 \\
228 \\
228 \\
230 \\
235\end{array}$ & $\begin{array}{r}17.98 \\
10.03 \\
5.80 \\
3.25 \\
8.75 \\
0.31\end{array}$ & $\begin{array}{l}299.6 \\
299.4 \\
299.3 \\
298.3 \\
294.4 \\
293.6\end{array}$ \\
\hline
\end{tabular}

$\$$

AVERAGES FOR 900 RECOROS OF 1 SECOND EACH ENDING ON JULIAN DAY 184 AT 024510

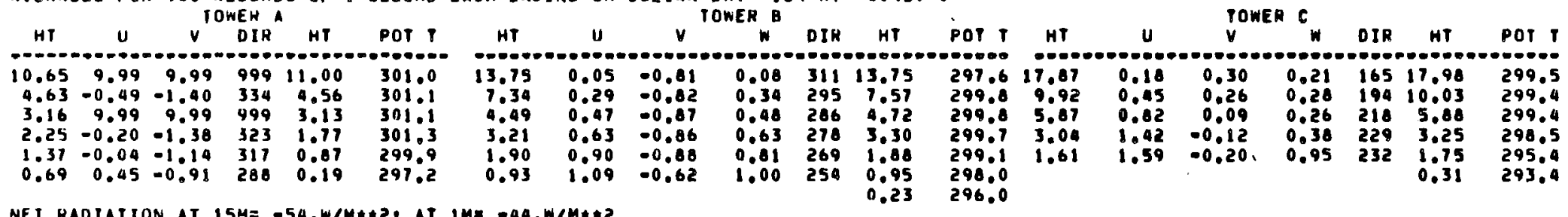

NET RADIATION AT 15M= -54.W/MHER, AT IME -44,W/M\#H2

AVEHAGES FOH 900 RECORDS OF I SECOND EACH ENDING ON JULIAN DAY 104 AT IT OL 0

HI
U


JULY 2-3, 1981: 1873-0345 PSI

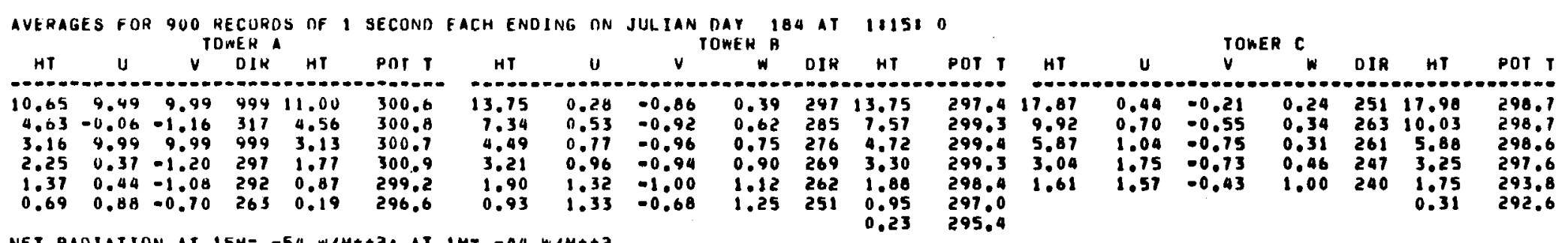

NET RADIATION AT 15M= -54.W/MMAZI AT IME -44.W/MHA2

AVERAGES FOR 900 RECORDS OF I SECOND EACH ENDING ON JULIAM OAY 184 AT 113080

\begin{tabular}{|c|c|c|c|c|c|c|c|c|c|c|c|c|c|c|c|c|c|c|c|}
\hline HT & If & $v$ & DIR & HT & Pot T & HT & u & $v$ & $w$ & DIR & HT & POT T & HT & $\mathbf{u}$ & $v$ & $w$ & DIR & HT & POT \\
\hline $\begin{array}{l}0.65 \\
4.63 \\
3.16 \\
2.25 \\
1.37 \\
0.69\end{array}$ & $\begin{array}{r}9.99 \\
-0.22 \\
9.99 \\
0.09 \\
0.15 \\
0.66\end{array}$ & $\begin{array}{r}9.99 \\
-1.67 \\
9.99 \\
-1.67 \\
-1.42 \\
-1.02\end{array}$ & $\begin{array}{l}999 \\
322 \\
999 \\
311 \\
304 \\
262\end{array}$ & $\begin{array}{r}11.00 \\
4.56 \\
3.13 \\
1.71 \\
0.87 \\
0.19\end{array}$ & $\begin{array}{l}301.0 \\
301.1 \\
301.1 \\
301.3 \\
299.7 \\
291.3\end{array}$ & $\begin{array}{r}13.75 \\
7.34 \\
4.49 \\
3.21 \\
1.90 \\
0.93\end{array}$ & $\begin{array}{l}0.15 \\
0.42 \\
0.64 \\
0.80 \\
1.06 \\
1.07\end{array}$ & $\begin{array}{l}-1.50 \\
-1.41 \\
-1.31 \\
-1.29 \\
-1.23 \\
-0.04\end{array}$ & $\begin{array}{l}0.34 \\
0.60 \\
0.69 \\
0.82 \\
1.00 \\
1.06\end{array}$ & $\begin{array}{l}309 \\
298 \\
289 \\
283 \\
274 \\
263\end{array}$ & $\begin{array}{r}13.75 \\
7.51 \\
9.72 \\
3.30 \\
1.80 \\
0.95 \\
0.23\end{array}$ & $\begin{array}{l}298.5 \\
299.8 \\
299.0 \\
299.7 \\
299.0 \\
298.1 \\
296.5\end{array}$ & $\begin{array}{r}17.87 \\
9.92 \\
5.87 \\
3.04 \\
1.61\end{array}$ & $\begin{array}{l}0.59 \\
0.76 \\
0.98 \\
1.55 \\
1.53\end{array}$ & $\begin{array}{l}-1.08 \\
-1.11 \\
-1.26 \\
-1.16 \\
-0.56\end{array}$ & $\begin{array}{l}0.28 \\
0.36 \\
0.28 \\
0.43 \\
0.99\end{array}$ & $\begin{array}{l}286 \\
282 \\
217 \\
261 \\
245\end{array}$ & $\begin{array}{r}17.90 \\
10.03 \\
5.88 \\
3.25 \\
1.75 \\
0.31\end{array}$ & $\begin{array}{l}299.6 \\
299.4 \\
299.4 \\
298.7 \\
294.9 \\
293.2\end{array}$ \\
\hline
\end{tabular}

AVERAGES FOH 900 RECORDS OF 1 SECOND EACH ENOING ON JULIAN DAY 184 AT 114510

\begin{tabular}{|c|c|c|c|c|c|c|c|c|c|c|c|c|c|c|c|c|c|c|c|}
\hline $\begin{array}{l}H T \\
H T\end{array}$ & $u$ & $v$ & OIR & $\begin{array}{c}H T \\
H\end{array}$ & POT T & HT & $u$ & $v$ & $w$ & DIR & HT & POT I & $\begin{array}{c}\text { HT } \\
-\infty\end{array}$ & $u$ & $v$ & 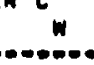 & DIR & $\begin{array}{c}\text { HT } \\
-0 . .0\end{array}$ & ד' \\
\hline $\begin{array}{l}10.65 \\
4.63 \\
3.16 \\
2.25 \\
1.37 \\
0.69\end{array}$ & $\begin{array}{r}9.99 \\
-0.30 \\
9.99 \\
0.10 \\
0.19 \\
0.68\end{array}$ & $\begin{array}{r}9.99 \\
-1.59 \\
9.99 \\
-1.58 \\
-1.34 \\
-0.96\end{array}$ & $\begin{array}{l}999 \\
325 \\
999 \\
311 \\
307 \\
279\end{array}$ & $\begin{array}{r}11.00 \\
4.56 \\
3.13 \\
1.77 \\
0.07 \\
0.19\end{array}$ & $\begin{array}{l}301.1 \\
301.1 \\
300.9 \\
301.1 \\
299.6 \\
291.4\end{array}$ & $\begin{array}{r}13.75 \\
7.34 \\
4.49 \\
3.21 \\
1.90 \\
0.93\end{array}$ & $\begin{array}{r}-0.34 \\
0.10 \\
0.42 \\
0.63 \\
0.92 \\
1.04\end{array}$ & $\begin{array}{l}-1.36 \\
-1.26 \\
-1.21 \\
-1.19 \\
-1.16 \\
-0.81\end{array}$ & $\begin{array}{r}0.13 \\
0.28 \\
0.49 \\
0.68 \\
0.88 \\
1.02\end{array}$ & $\begin{array}{l}329 \\
310 \\
296 \\
287 \\
276 \\
262\end{array}$ & $\begin{array}{r}13.75 \\
7.57 \\
4.72 \\
3.30 \\
1.80 \\
0.95 \\
0.23\end{array}$ & $\begin{array}{l}298.3 \\
299.8 \\
299.6 \\
299.5 \\
298.8 \\
298.0 \\
296.4\end{array}$ & $\begin{array}{r}17.87 \\
9.92 \\
5.87 \\
3.04 \\
1.61\end{array}$ & $\begin{array}{l}0.33 \\
0.65 \\
0.99 \\
1.46 \\
1.42\end{array}$ & $\begin{array}{l}-1.35 \\
-1.46 \\
-1.55 \\
-1.40 \\
-0.88\end{array}$ & $\begin{array}{l}0.24 \\
0.35 \\
0.26 \\
0.42 \\
0.95\end{array}$ & $\begin{array}{l}301 \\
290 \\
282 \\
260 \\
256\end{array}$ & $\begin{array}{r}17.98 \\
10.03 \\
5.88 \\
3.25 \\
1.75 \\
0.31\end{array}$ & $\begin{array}{l}299.8 \\
299.6 \\
299.4 \\
298.7 \\
295.6 \\
293.7\end{array}$ \\
\hline
\end{tabular}

NET RAOIATION AT 15ME -54.W/M\#\#2, AT IME -44,W/M\#MZ

AVERAGES FOR 900 RECONOS OF I SECOND EACH ENOING ON JULIAN OAY 184 AT 21080

\begin{tabular}{|c|c|c|c|c|c|c|c|c|c|c|c|c|c|c|c|c|c|c|c|}
\hline HT & u & $v$ & DIR & $H T$ & POT $T$ & HT & $u$ & $v$ & 1 & DIA & HT & Por 1 & HT & $\mathbf{u}$ & $v^{T D t}$ & ${ }^{c}$ & OIR & HT & POT \\
\hline $\begin{array}{r}10.65 \\
4.63 \\
3.16 \\
2.25 \\
1.37 \\
0.69\end{array}$ & $\begin{array}{l}9.99 \\
0.04 \\
9.99 \\
0.51 \\
0.60 \\
1.00\end{array}$ & $\begin{array}{r}9.99 \\
-0.99 \\
9.99 \\
-1.07 \\
-1.01 \\
-0.00\end{array}$ & $\begin{array}{l}999 \\
312 \\
499 \\
289 \\
284 \\
255\end{array}$ & $\begin{array}{r}11.00 \\
4.56 \\
3.13 \\
1.77 \\
0.87 \\
0.19\end{array}$ & $\begin{array}{l}300.9 \\
301.0 \\
300.9 \\
301.1 \\
299.4 \\
296.6\end{array}$ & $\begin{array}{r}13.75 \\
1.34 \\
4.49 \\
3.21 \\
1.90 \\
0.93\end{array}$ & $\begin{array}{l}0.05 \\
0.41 \\
0.68 \\
0.88 \\
1.20 \\
1.27\end{array}$ & $\begin{array}{l}-1.07 \\
-1.02 \\
-1.02 \\
-0.91 \\
-1.00 \\
-0.067\end{array}$ & $\begin{array}{l}0.26 \\
0.53 \\
0.68 \\
0.84 \\
1.04 \\
1.18\end{array}$ & $\begin{array}{l}312 \\
292 \\
281 \\
212 \\
264 \\
252\end{array}$ & $\begin{array}{l}13.75 \\
7.51 \\
4.72 \\
3.30 \\
1.88 \\
0.95 \\
0.23\end{array}$ & $\begin{array}{l}290.0 \\
299.1 \\
299.6 \\
299.6 \\
298.9 \\
297.5 \\
295.7\end{array}$ & $\begin{array}{r}17.87 \\
9.92 \\
5.87 \\
3.04 \\
1.61\end{array}$ & $\begin{array}{l}0.48 \\
0.90 \\
1.24 \\
1.82 \\
1.63\end{array}$ & $\begin{array}{l}-1.34 \\
-1.42 \\
-1.43 \\
-1.23 \\
-0.63\end{array}$ & $\begin{array}{l}0.26 \\
0.40 \\
0.34 \\
0.53 \\
1.06\end{array}$ & $\begin{array}{l}295 \\
282 \\
274 \\
259 \\
246\end{array}$ & $\begin{array}{l}17.98 \\
10.03 \\
5.88 \\
3.25 \\
1.75 \\
0.31\end{array}$ & $\begin{array}{l}299.0 \\
299.5 \\
299.4 \\
298.5 \\
295.1 \\
293.1\end{array}$ \\
\hline
\end{tabular}


JULY 2-3, 1981: 1813-0345 PST

AVEKAGES FOH 90O RECUHIS OF I SECOHO EACH ENDING ON JULIAN DAY 184 AT 211580

\begin{tabular}{|c|c|c|c|c|c|c|c|c|c|c|c|c|c|c|c|c|c|c|c|}
\hline HT & $u$ & $v^{\prime \prime}$ & $\begin{array}{l}\text { NEK } \\
\text { OIK }\end{array}$ & HI & Por 1 & $H T$ & $u$ & $v$ & $\mathrm{KER}^{\mathrm{N}}{ }^{\mathrm{B}}$ & otk & HI & Pot 8 & HI & u & $\begin{array}{l}\text { ro } \\
v\end{array}$ & C C & DIR & HT & Por \\
\hline $\begin{array}{r}10.65 \\
4.63 \\
3.16 \\
2.25 \\
1.37 \\
0.69\end{array}$ & $\begin{array}{l}9.99 \\
0.24 \\
9.99 \\
0.83 \\
0.90 \\
1.30\end{array}$ & $\begin{array}{r}9.99 \\
-0.40 \\
9.99 \\
-0.52 \\
-0.05 \\
-0.34\end{array}$ & $\begin{array}{l}994 \\
288 \\
999 \\
257 \\
261 \\
239\end{array}$ & $\begin{array}{r}11.00 \\
4.56 \\
3.13 \\
1.77 \\
0.07 \\
0.19\end{array}$ & $\begin{array}{l}301.2 \\
301.4 \\
301.3 \\
301.5 \\
299.7 \\
295.9\end{array}$ & $\begin{array}{r}13.75 \\
7.34 \\
4.49 \\
3.21 \\
1.90 \\
0.93\end{array}$ & $\begin{array}{l}0.35 \\
0.56 \\
0.84 \\
1.04 \\
1.39 \\
1.44\end{array}$ & $\begin{array}{l}-0.83 \\
-0.82 \\
-0.88 \\
=0.86 \\
=0.90 \\
-0.56\end{array}$ & $\begin{array}{l}0.45 \\
0.64 \\
0.80 \\
0.94 \\
1.16 \\
1.31\end{array}$ & $\begin{array}{l}293 \\
280 \\
271 \\
264 \\
258 \\
246\end{array}$ & $\begin{array}{r}13.75 \\
7.57 \\
4.72 \\
3.30 \\
1.80 \\
0.95 \\
0.23\end{array}$ & $\begin{array}{l}290.3 \\
300.0 \\
299.9 \\
299.9 \\
299.0 \\
297.1 \\
295.4\end{array}$ & $\begin{array}{r}17.87 \\
9.92 \\
5.87 \\
3.04 \\
1.61\end{array}$ & $\begin{array}{l}0.85 \\
1.13 \\
1.46 \\
2.22 \\
1.73\end{array}$ & $\begin{array}{l}-1.39 \\
-1.44 \\
-1.43 \\
-1.25 \\
-0.66\end{array}$ & $\begin{array}{l}0.32 \\
0.43 \\
0.36 \\
0.65 \\
1.13\end{array}$ & $\begin{array}{l}203 \\
276 \\
269 \\
254 \\
245\end{array}$ & $\begin{array}{r}17.98 \\
10.03 \\
5.88 \\
3.25 \\
1.75 \\
0.31\end{array}$ & $\begin{array}{l}300.1 \\
299.8 \\
299.6 \\
298.3 \\
294.7 \\
293.7\end{array}$ \\
\hline
\end{tabular}

NET RADIATION AT 15M= -54,W/MMAC, AT IME MMA,W/MAMZ

AVERAGES FOH 900 RECOROS OF 1 SECONO EACH ENDING ON JULIAN DAY 104 AT 213020

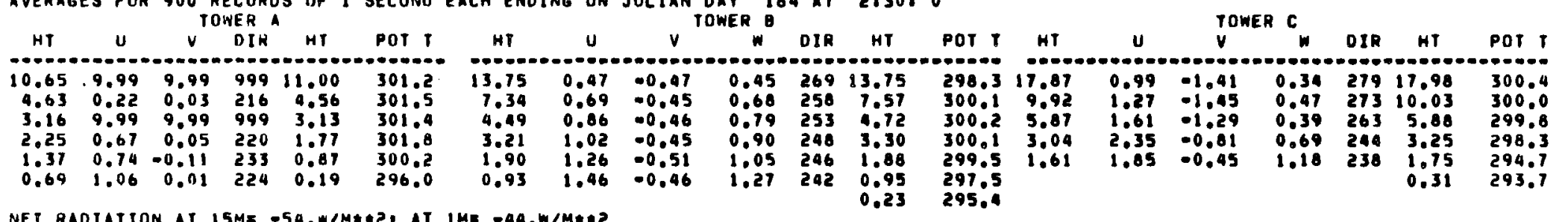


JULY 2-3, 1981: 1813-0345 PST

\begin{tabular}{|c|c|c|c|c|c|c|c|c|c|c|c|c|c|c|c|c|c|c|c|}
\hline $\begin{array}{l}\text { AVEHAGES } \\
\text { HI }\end{array}$ & $\begin{array}{l}\text { S FOH } \\
\text { u }\end{array}$ & $v^{198}$ & $\begin{array}{l}\text { ECORDS } \\
\text { WER } \\
\text { DIR }\end{array}$ & S OF 1 & $\begin{array}{l}\text { SECONI) } \\
\text { POT T }\end{array}$ & $\begin{array}{c}\text { EACH ENDING } \\
\text { HT }\end{array}$ & 11 & $\begin{array}{c}\text { JIILIAN } \\
V\end{array}$ & $\begin{array}{ll}Y Y \\
\text { UER }\end{array}$ & UIR & $\begin{array}{l}380 \\
\text { HT }\end{array}$ & POT T & HT & 0 & TOH & c & DIR & HT & POT T \\
\hline $\begin{array}{l}10.65 \\
4.63 \\
3.16 \\
2.25 \\
1.31 \\
0.69\end{array}$ & $\begin{array}{l}9.99 \\
0.16 \\
9.99 \\
1.30 \\
1.56 \\
1.56\end{array}$ & $\begin{array}{l}9.99 \\
0.91 \\
9.99 \\
0.83 \\
0.45 \\
0.31\end{array}$ & $\begin{array}{l}999 \\
174 \\
999 \\
192 \\
2118 \\
211\end{array}$ & $\begin{array}{r}11.00 \\
4.56 \\
3.13 \\
1.77 \\
0.87 \\
0.19\end{array}$ & $\begin{array}{l}300.7 \\
300.8 \\
300.8 \\
301.0 \\
299.0 \\
295.9\end{array}$ & $\begin{array}{r}13.75 \\
1.34 \\
4.49 \\
3.21 \\
1.90 \\
0.93\end{array}$ & $\begin{array}{l}0.32 \\
0.71 \\
1.04 \\
1.30 \\
1.61 \\
1.45\end{array}$ & $\begin{array}{l}0.87 \\
0.82 \\
0.65 \\
0.67 \\
0.41 \\
0.00\end{array}$ & $\begin{array}{l}0.29 \\
0.64 \\
0.91 \\
1.01 \\
1.22 \\
1.29\end{array}$ & $\begin{array}{l}154 \\
176 \\
193 \\
197 \\
210 \\
224\end{array}$ & $\begin{array}{r}13.75 \\
7.57 \\
4.72 \\
3.30 \\
1.88 \\
0.95 \\
0.23\end{array}$ & $\begin{array}{l}297.1 \\
299.5 \\
299.5 \\
299.5 \\
290.5 \\
297.0 \\
295.6\end{array}$ & $\begin{array}{r}17.87 \\
9.92 \\
5.87 \\
3.04 \\
1.61\end{array}$ & $\begin{array}{l}0.56 \\
0.97 \\
1.37 \\
1.92 \\
1.70\end{array}$ & $\begin{array}{r}1.03 \\
0.80 \\
0.69 \\
0.41 \\
-0.06\end{array}$ & $\begin{array}{l}0.27 \\
0.39 \\
0.34 \\
0.51 \\
1.06\end{array}$ & $\begin{array}{l}163 \\
185 \\
198 \\
213 \\
226\end{array}$ & $\begin{array}{r}17.98 \\
10.03 \\
5.88 \\
3.25 \\
1.75 \\
0.31\end{array}$ & $\begin{array}{l}299.4 \\
299.2 \\
299.2 \\
298.3 \\
295.2 \\
293.6\end{array}$ \\
\hline
\end{tabular}

NET HADIATION AT $15 \mathrm{MZ} 126$. W/M\#\#2, AT $1 \mathrm{MZ} 177$.W/M\#\#2

AVERAGES FOR 900 RECORDS OF 1 SECOND EACH ENDING ON JULIAN DAY 184 AT 311580

\begin{tabular}{|c|c|c|c|c|c|c|c|c|c|c|c|c|c|c|c|c|c|c|c|}
\hline HT & "I & $v$ & DIR & HT & POT T & HT & U & $v$ & $w$ & DIR & HI & POT 1 & HT & u & $v$ & $w$ & DIR & $\mathrm{HT}$ & POT \\
\hline $\begin{array}{l}10.65 \\
4.03 \\
3.16 \\
2.25 \\
1.37 \\
0.09\end{array}$ & $\begin{array}{l}9.99 \\
0.66 \\
9.99 \\
1.14 \\
1.34 \\
1.54\end{array}$ & $\begin{array}{l}9.99 \\
0.38 \\
9.99 \\
0.34 \\
0.03 \\
0.08\end{array}$ & $\begin{array}{l}9991 \\
195 \\
999 \\
208 \\
223 \\
221\end{array}$ & $\begin{array}{r}11.00 \\
4.56 \\
3.13 \\
1.177 \\
0.87 \\
0.19\end{array}$ & $\begin{array}{l}300.8 \\
301.1 \\
301.0 \\
301.2 \\
299.2 \\
295.9\end{array}$ & $\begin{array}{r}13.75 \\
7.34 \\
4.49 \\
3.21 \\
1.90 \\
0.93\end{array}$ & $\begin{array}{l}0.27 \\
0.51 \\
0.87 \\
1.10 \\
1.45 \\
1.45\end{array}$ & $\begin{array}{r}0.69 \\
0.61 \\
0.46 \\
0.39 \\
0.12 \\
-0.19\end{array}$ & $\begin{array}{l}0.30 \\
0.48 \\
0.76 \\
0.90 \\
1.12 \\
1.27\end{array}$ & $\begin{array}{l}156 \\
174 \\
197 \\
205 \\
220 \\
232\end{array}$ & $\begin{array}{r}13.75 \\
7.57 \\
4.72 \\
3.30 \\
1.80 \\
0.95 \\
0.23\end{array}$ & $\begin{array}{l}297.1 \\
299.6 \\
299.5 \\
299.4 \\
298.5 \\
296.7 \\
295.4\end{array}$ & $\begin{array}{r}17.67 \\
9.92 \\
5.87 \\
3.04 \\
1.61\end{array}$ & $\begin{array}{l}0.44 \\
0.97 \\
1.33 \\
1.60 \\
1.43\end{array}$ & $\begin{array}{l}1.52 \\
1.40 \\
1.17 \\
0.71 \\
0.21\end{array}$ & $\begin{array}{l}0.26 \\
0.36 \\
0.27 \\
0.38 \\
0.85\end{array}$ & $\begin{array}{l}151 \\
169 \\
183 \\
201 \\
216\end{array}$ & $\begin{array}{r}17.98 \\
10.03 \\
5.80 \\
3.25 \\
1.75 \\
0.31\end{array}$ & $\begin{array}{l}298.4 \\
298.2 \\
298.1 \\
291.3 \\
295.2 \\
293.5\end{array}$ \\
\hline
\end{tabular}

NET RAOIATION AT 15M= -23.W/MH*2I AT IME T.W/MHA2

$\pm$

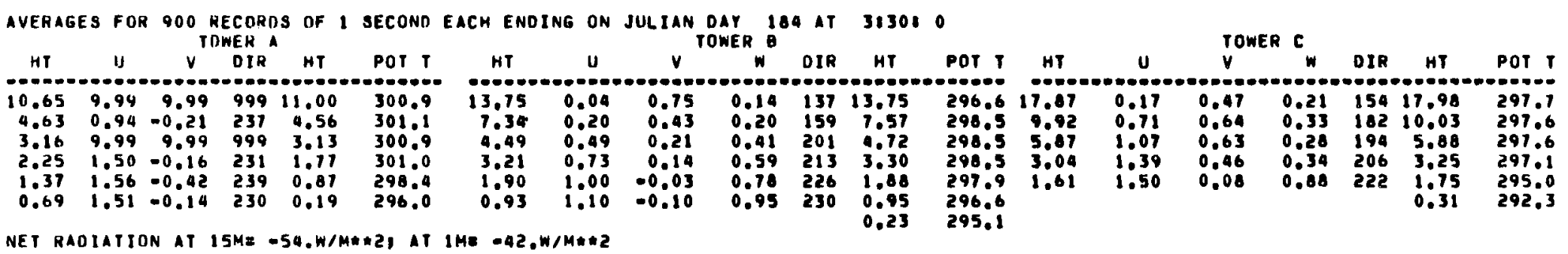

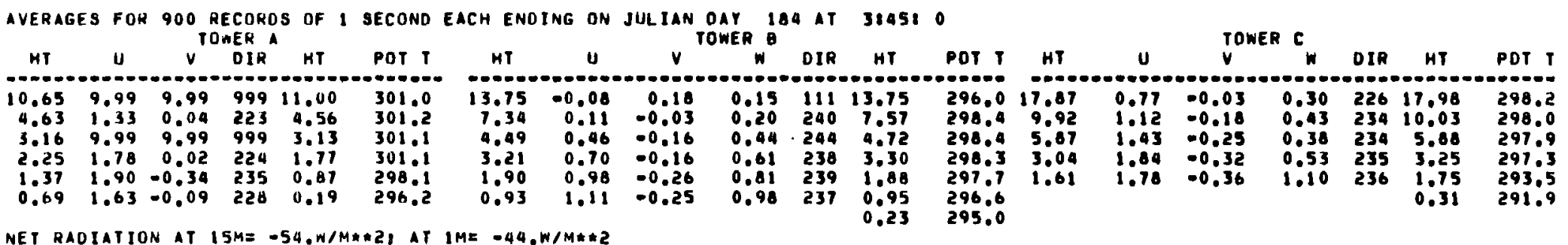

NET RAOIATION AT ISM= -54,W/MA*2, AT IME -44,W/M\#\#2 
OCTOBER 16, 1981; 1510-2215 PST

AVEHAGES FOR 328 RECIHHS OF I SECOHAD EACH EIHAING ON JULIAN DAY 289 AT 151530

\begin{tabular}{|c|c|c|c|c|c|c|c|c|c|c|c|c|c|c|c|c|c|c|c|}
\hline HT & $u$ & $v$ & $\mathrm{OIH}$ & HI & POT & $\mathrm{HT}$ & 11 & $v$ & $w$ & OIR & HT & POT & $H T$ & $\mathbf{u}$ & $v$ & $w$ & DIR & HT & POT \\
\hline $\begin{array}{r}10.65 \\
4.63 \\
3.16 \\
2.25 \\
1.37 \\
0.69\end{array}$ & $\begin{array}{l}9.99 \\
3.21 \\
9.99 \\
2.70 \\
1.66 \\
1.97\end{array}$ & $\begin{array}{r}9.99 \\
-0.09 \\
9.99 \\
-0.13 \\
-0.29 \\
0.11\end{array}$ & $\begin{array}{l}999 \\
226 \\
999 \\
227 \\
235 \\
221\end{array}$ & $\begin{array}{r}11.00 \\
4.56 \\
3.13 \\
1.77 \\
0.87 \\
0.19\end{array}$ & $\begin{array}{l}292.5 \\
292.8 \\
292.8 \\
292.9 \\
292.9 \\
292.1\end{array}$ & $\begin{array}{r}13.75 \\
7.34 \\
4.49 \\
3.21 \\
1.40 \\
0.93\end{array}$ & $\begin{array}{l}3.18 \\
2.53 \\
2.31 \\
2.28 \\
1.85 \\
1.43\end{array}$ & $\begin{array}{l}-0.90 \\
-0.78 \\
-0.76 \\
=0.72 \\
-0.77 \\
-0.41\end{array}$ & $\begin{array}{l}2.41 \\
2.39 \\
2.03 \\
1.13 \\
1.49 \\
1.38\end{array}$ & $\begin{array}{l}240 \\
242 \\
243 \\
242 \\
247 \\
241\end{array}$ & $\begin{array}{r}13.75 \\
7.57 \\
4.72 \\
3.30 \\
1.88 \\
0.95 \\
0.23\end{array}$ & $\begin{array}{l}292.0 \\
292.0 \\
292.1 \\
292.0 \\
291.8 \\
999.9 \\
291.0\end{array}$ & $\begin{array}{r}17.87 \\
9.92 \\
5.87 \\
3.04 \\
1.61\end{array}$ & $\begin{array}{l}5.00 \\
4.26 \\
3.86 \\
3.15 \\
2.70\end{array}$ & $\begin{array}{l}0.84 \\
0.50 \\
0.47 \\
0.36 \\
0.15\end{array}$ & $\begin{array}{l}0.98 \\
0.97 \\
1.04 \\
0,80 \\
0.85\end{array}$ & $\begin{array}{l}215 \\
218 \\
218 \\
218 \\
221\end{array}$ & $\begin{array}{r}17.98 \\
10.03 \\
5.88 \\
3.25 \\
1 . .75 \\
0.31\end{array}$ & $\begin{array}{l}292.6 \\
292.5 \\
292.6 \\
292.7 \\
292.6 \\
293.8\end{array}$ \\
\hline
\end{tabular}

AVERAGES FOR 900 RECBRDS OF I SECOND EACH ENDING ON JULIAN DAY 289 AT 1583010

\begin{tabular}{|c|c|c|c|c|c|c|c|c|c|c|c|c|c|c|c|c|c|c|c|}
\hline & & & WER & & & & & & 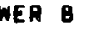 & & & & & & & & & & \\
\hline $\mathrm{HT}$ & u & $v$ & & HT & T & & U & & $\omega$ & IR & & Tot & HT & & & & DIR & HT & POT $T$ \\
\hline $\begin{array}{l}10.65 \\
4.63 \\
3.16 \\
2.25 \\
1.37 \\
0.69\end{array}$ & $\begin{array}{l}9.99 \\
3.01 \\
9.99 \\
2.49 \\
1.50 \\
1.75\end{array}$ & $\begin{array}{l}9.99 \\
0.69 \\
9.99 \\
0.60 \\
0.19 \\
0.63\end{array}$ & $\begin{array}{l}999 \\
212 \\
999 \\
211 \\
217 \\
205\end{array}$ & $\begin{array}{r}11.00 \\
4.56 \\
3.13 \\
1.77 \\
0.87 \\
0.19\end{array}$ & $\begin{array}{l}292.5 \\
292.8 \\
292.8 \\
292.8 \\
292.8 \\
292.1\end{array}$ & $\begin{array}{r}13.75 \\
7.34 \\
4.49 \\
3.21 \\
1.90 \\
0.93\end{array}$ & $\begin{array}{l}3.30 \\
2.42 \\
2.22 \\
2.17 \\
1.80 \\
1.32\end{array}$ & $\begin{array}{l}0.36 \\
0.51 \\
0.40 \\
0.41 \\
0.19 \\
0.34\end{array}$ & $\begin{array}{l}2.48 \\
2.28 \\
1.93 \\
1.09 \\
1.31 \\
1.23\end{array}$ & $\begin{array}{l}210 \\
213 \\
219 \\
219 \\
218 \\
210\end{array}$ & $\begin{array}{l}13.75 \\
7.57 \\
4.72 \\
3.30 \\
1.88 \\
0.95 \\
0.93\end{array}$ & $\begin{array}{l}291.8 \\
291.8 \\
298.8 \\
291.8 \\
291.6 \\
999.9 \\
291.1\end{array}$ & $\begin{array}{r}17.87 \\
9.92 \\
5.87 \\
3.04 \\
1.61\end{array}$ & $\begin{array}{l}5.46 \\
4.03 \\
4.37 \\
3.46 \\
2.09\end{array}$ & $\begin{array}{l}0.70 \\
0.56 \\
0.57 \\
0.45 \\
0.18\end{array}$ & $\begin{array}{l}1.07 \\
1.04 \\
1.10 \\
0.93 \\
0.91\end{array}$ & $\begin{array}{l}217 \\
218 \\
217 \\
217 \\
221\end{array}$ & $\begin{array}{r}17.98 \\
10.03 \\
5.88 \\
3.25 \\
1.75 \\
0.31\end{array}$ & $\begin{array}{l}292.4 \\
292.02 \\
292.3 \\
292.3 \\
292.1 \\
293.1\end{array}$ \\
\hline
\end{tabular}

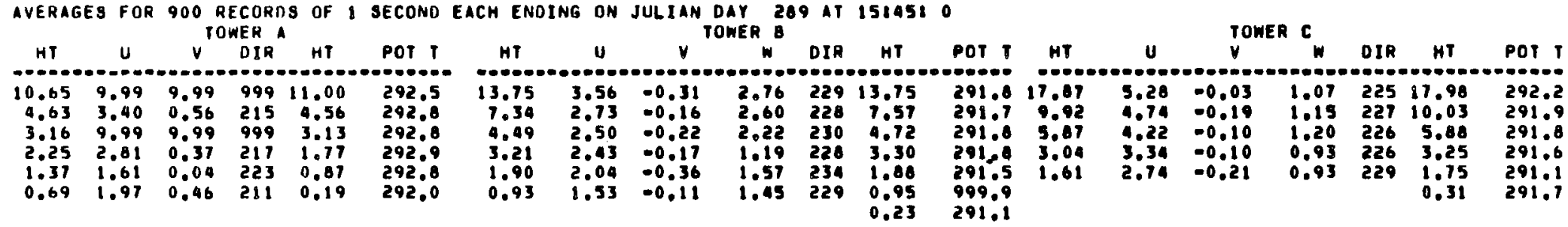

AVERAGES FOR 900 RECORDS OF 1 SECONO EACH ENDING ON JULIAN DAY 209 AT 168 O8 0

\begin{tabular}{|c|c|c|c|c|c|c|c|c|c|c|c|c|c|c|c|c|c|c|c|}
\hline HT & u & $v$ & DIR & HT & Pot T & HT & 11 & $v$ & W & DIR & HT & POT & HT & $\mathbf{U}$ & $v$ & $w$ & DIR & HT & PDT T \\
\hline $\begin{array}{r}10.65 \\
4.63 \\
3.16 \\
2.25 \\
1.37 \\
0.69\end{array}$ & $\begin{array}{l}9.99 \\
4.12 \\
9.99 \\
3.37 \\
1.89 \\
2.38\end{array}$ & $\begin{array}{r}9.99 \\
0.38 \\
9.98 \\
0.18 \\
-0.11 \\
0.42\end{array}$ & $\begin{array}{l}999 \\
219 \\
999 \\
221 \\
228 \\
215\end{array}$ & $\begin{array}{r}11.00 \\
4.56 \\
3.13 \\
1.17 \\
0.87 \\
0.19\end{array}$ & $\begin{array}{l}292.5 \\
292.8 \\
292.8 \\
292.9 \\
292.8 \\
292.0\end{array}$ & $\begin{array}{r}13.75 \\
7.34 \\
9.49 \\
3.21 \\
1.90 \\
0.93\end{array}$ & $\begin{array}{l}4.11 \\
3.12 \\
2.17 \\
2.73 \\
2.22 \\
1.68\end{array}$ & $\begin{array}{r}-0.12 \\
0.03 \\
-0.04 \\
0.04 \\
-0.22 \\
0.01\end{array}$ & $\begin{array}{l}3.12 \\
2.90 \\
2.44 \\
1.30 \\
1.69 \\
1.60\end{array}$ & $\begin{array}{l}226 \\
224 \\
225 \\
224 \\
230 \\
224\end{array}$ & $\begin{array}{r}13.75 \\
7.57 \\
4.72 \\
3.30 \\
1.80 \\
0.95 \\
0.23\end{array}$ & $\begin{array}{l}291.8 \\
291.7 \\
291.7 \\
291.7 \\
291.5 \\
999.9 \\
291.0\end{array}$ & $\begin{array}{r}17.87 \\
9.92 \\
5.87 \\
3.04 \\
1.61\end{array}$ & $\begin{array}{l}5.90 \\
5.19 \\
.64 \\
3.60 \\
3.04\end{array}$ & $\begin{array}{l}0.51 \\
0.30 \\
0.28 \\
0.24 \\
0.05\end{array}$ & $\begin{array}{l}1.16 \\
8.14 \\
1.23 \\
0.99 \\
0.95\end{array}$ & $\begin{array}{l}220 \\
221 \\
221 \\
221 \\
224\end{array}$ & $\begin{array}{r}17.98 \\
10.03 \\
5.08 \\
3.25 \\
1.75 \\
0.31\end{array}$ & $\begin{array}{l}291.8 \\
291.6 \\
291.5 \\
291.4 \\
290.9 \\
291.4\end{array}$ \\
\hline
\end{tabular}


OCTOBER 16, 1981; 1510-2215 PST

AVEHAGES FOH 900 HECORUS OF 1 SECOHD EACH ENDING ON JULIAN DAY 289 AT 1681580

\begin{tabular}{|c|c|c|c|c|c|c|c|c|c|c|c|c|c|c|c|c|c|c|c|}
\hline $\mathrm{HT}$ & u & $v$ & $\begin{array}{l}N E R \\
D I R\end{array}$ & HI & POI I & HT & II & $v$ & $w$ & UIR & HI & POT 1 & HT & U & $\begin{array}{l}\text { Tow } \\
v\end{array}$ & $\mathrm{C}_{\mathrm{W}}$ & DIR & HT & Por \\
\hline $\begin{array}{l}10.65 \\
4.63 \\
3.16 \\
2.25 \\
1.37 \\
0.69\end{array}$ & $\begin{array}{l}9.99 \\
4.72 \\
9.99 \\
3.92 \\
2.23 \\
2.02\end{array}$ & $\begin{array}{r}9.99 \\
0.37 \\
9.99 \\
0.19 \\
-0.15 \\
0.39\end{array}$ & $\begin{array}{l}999 \\
220 \\
999 \\
222 \\
228 \\
217\end{array}$ & $\begin{array}{r}11.00 \\
4.30 \\
3.13 \\
1.77 \\
0.87 \\
0.19\end{array}$ & $\begin{array}{l}292.5 \\
292.7 \\
292.7 \\
292.8 \\
292.8 \\
291.9\end{array}$ & $\begin{array}{r}13.75 \\
1.34 \\
4.49 \\
3.21 \\
1.90 \\
0.93\end{array}$ & $\begin{array}{l}4.81 \\
3.71 \\
3.42 \\
3.38 \\
2.79 \\
2.09\end{array}$ & $\begin{array}{l}=0.56 \\
-0.33 \\
-0.37 \\
-0.25 \\
-0.48 \\
-0.24\end{array}$ & $\begin{array}{l}3.79 \\
3.52 \\
3.01 \\
1.55 \\
2.19 \\
1.98\end{array}$ & $\begin{array}{l}231 \\
230 \\
231 \\
229 \\
234 \\
231\end{array}$ & $\begin{array}{l}13.75 \\
7.51 \\
4.72 \\
3.30 \\
1.88 \\
0.95 \\
0.23\end{array}$ & $\begin{array}{l}291.0 \\
291.7 \\
291.7 \\
291.7 \\
291.5 \\
999.9 \\
291.0\end{array}$ & $\begin{array}{r}17.87 \\
9.92 \\
5.87 \\
3.04 \\
1.61\end{array}$ & $\begin{array}{l}6.86 \\
5.94 \\
5.21 \\
4.11 \\
3.38\end{array}$ & $\begin{array}{r}0.09 \\
-0.09 \\
-0.09 \\
-0.08 \\
-0.27\end{array}$ & $\begin{array}{l}1.32 \\
1.30 \\
1.34 \\
1.09 \\
1.06\end{array}$ & $\begin{array}{l}224 \\
225 \\
225 \\
226 \\
229\end{array}$ & $\begin{array}{r}17.98 \\
10.03 \\
5.88 \\
3.25 \\
1.75 \\
0.31\end{array}$ & $\begin{array}{l}291.8 \\
291.5 \\
291.5 \\
291.3 \\
290.8 \\
291.3\end{array}$ \\
\hline
\end{tabular}

AVERAGES FOR 900 HECOROS OF 1 SECOND EACH ENDING ON JULIAN DAY 289 AT 1613080

\begin{tabular}{|c|c|c|c|c|c|c|c|c|c|c|c|c|c|c|c|c|c|c|c|}
\hline & & & NER A & & & & & & R $B$ & & & & & & & C & & & \\
\hline HT & u & $v$ & $k$ & HT & POT T & & u & $v$ & & DIR & HT & T T & HT & U & & $w$ & OIR & & POT \\
\hline $\begin{array}{l}0.65 \\
4.63 \\
3.16 \\
2.25 \\
1.37 \\
0.69\end{array}$ & $\begin{array}{l}9.99 \\
4.62 \\
9.99 \\
3.81 \\
2.19 \\
2.76\end{array}$ & $\begin{array}{r}9.99 \\
-0.11 \\
9.99 \\
-0.17 \\
-0.36 \\
0.16\end{array}$ & $\begin{array}{l}999 \\
226 \\
999 \\
227 \\
234 \\
221\end{array}$ & $\begin{array}{r}11.00 \\
4.56 \\
3.13 \\
1.77 \\
0.87 \\
0.19\end{array}$ & $\begin{array}{l}292.5 \\
292.6 \\
292.6 \\
292.8 \\
292.7 \\
291.9\end{array}$ & $\begin{array}{r}13.75 \\
7.34 \\
4.49 \\
3.21 \\
1.90 \\
0.93\end{array}$ & $\begin{array}{l}5.13 \\
3.98 \\
3.54 \\
3.52 \\
2.89 \\
2.22\end{array}$ & $\begin{array}{l}-1.12 \\
-0.81 \\
-0.79 \\
-0.69 \\
-0.82 \\
-0.54\end{array}$ & $\begin{array}{l}4.07 \\
3.77 \\
3.17 \\
1.60 \\
2.31 \\
2.11\end{array}$ & $\begin{array}{l}237 \\
236 \\
231 \\
236 \\
240 \\
238\end{array}$ & $\begin{array}{r}13.75 \\
7.57 \\
4.72 \\
3.30 \\
1.86 \\
0.95 \\
0.23\end{array}$ & $\begin{array}{l}291.08 \\
291.7 \\
291.7 \\
291.6 \\
291.5 \\
999 . .9\end{array}$ & $\begin{array}{r}17.87 \\
9.92 \\
5.87 \\
3.04 \\
1.61\end{array}$ & $\begin{array}{l}7.73 \\
6.68 \\
3.89 \\
4.59 \\
3.80\end{array}$ & $\begin{array}{l}=0 \\
=0 \\
=0 \\
=0\end{array}$ & $\begin{array}{l}1.45 \\
1.50 \\
1.57 \\
1.24 \\
1.25\end{array}$ & $\begin{array}{l}228 \\
230 \\
230 \\
231 \\
234\end{array}$ & $\begin{array}{r}17.98 \\
10.03 \\
5.80 \\
3.25 \\
1.75 \\
0.31\end{array}$ & $\begin{array}{l}291.9 \\
291.6 \\
291.5 \\
291.5 \\
290.8 \\
291.3\end{array}$ \\
\hline
\end{tabular}

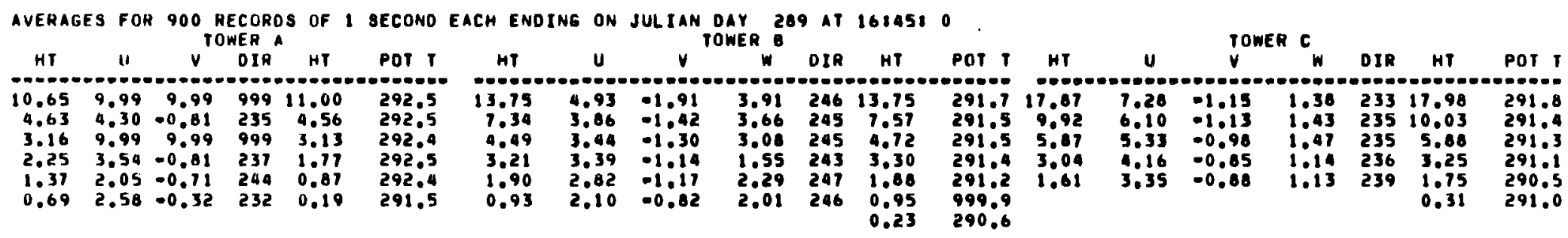

AVERAGES FOR 900 RECORDS OF I SECONO EACH ENDING ON JULIAN DAY 209 AT 111080

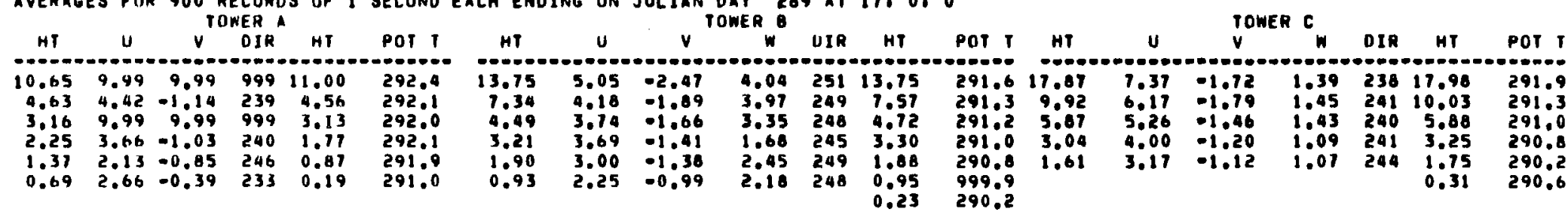




\section{OCTOBER 16, 1981; 1510-2215 PST}

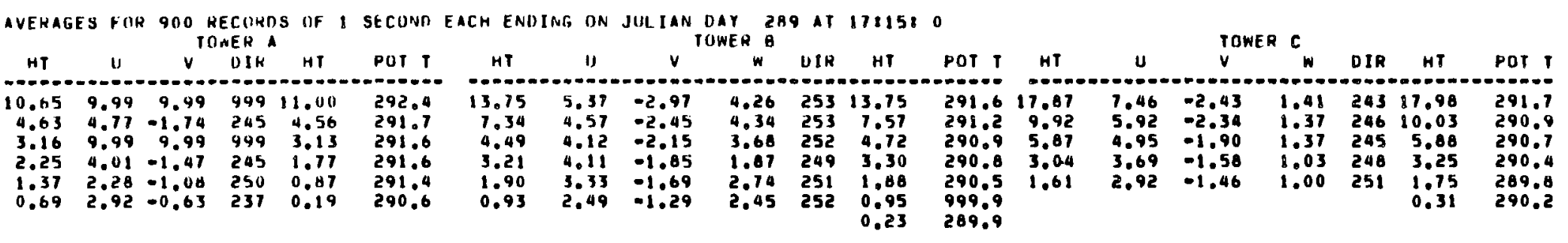

AVERAGES FOR 900 RECURDS OF 1 SECOND EACH ENDING ON JULIAN DAY 289 AT 1113010

\begin{tabular}{|c|c|c|c|c|c|c|c|c|c|c|c|c|c|c|c|c|c|c|c|}
\hline HT & u & $v$ & DIR & HT & POT $T$ & HT & u & $v$ & $w$ & DIR & HT & POT & HT & u & vor & $c_{w}$ & DIR & HT & POT \\
\hline $\begin{array}{l}0.65 \\
4.63 \\
3.16 \\
2.25 \\
1.37 \\
0.69\end{array}$ & $\begin{array}{l}9.99 \\
4.67 \\
9.99 \\
3.92 \\
2.19 \\
2.85\end{array}$ & $\begin{array}{r}9.99 \\
-2.27 \\
9.99 \\
-1.91 \\
-1.29 \\
-0.86\end{array}$ & $\begin{array}{l}999 \\
250 \\
999 \\
250 \\
255 \\
241\end{array}$ & $\begin{array}{r}11.00 \\
4.56 \\
3.113 \\
1.17 \\
0.87 \\
0.19\end{array}$ & $\begin{array}{l}292.4 \\
291.7 \\
291.5 \\
291.4 \\
291.1 \\
290.3\end{array}$ & $\begin{array}{r}13.15 \\
7.34 \\
4.49 \\
3.21 \\
1.90 \\
0.93\end{array}$ & $\begin{array}{l}5.19 \\
4.72 \\
4.33 \\
4.29 \\
3.51 \\
2.55\end{array}$ & $\begin{array}{l}-3.32 \\
-2.99 \\
-2.58 \\
-2.15 \\
-1.99 \\
-1.91\end{array}$ & $\begin{array}{l}4.19 \\
4.50 \\
3.89 \\
1.96 \\
2.91 \\
2.50\end{array}$ & $\begin{array}{l}257 \\
257 \\
255 \\
251 \\
253 \\
253\end{array}$ & $\begin{array}{r}13.75 \\
7.57 \\
4.72 \\
3.30 \\
1.86 \\
0.95 \\
0.23\end{array}$ & $\begin{array}{l}291.6 \\
291.04 \\
290.9 \\
290.7 \\
290.3 \\
999.9 \\
289.7\end{array}$ & $\begin{array}{l}17.87 \\
9.92 \\
9.87 \\
3.04 \\
1.61\end{array}$ & $\begin{array}{l}7.06 \\
5.61 \\
4.63 \\
3.53 \\
2.75\end{array}$ & $\begin{array}{l}-2.98 \\
-2.73 \\
-2.25 \\
-1.85 \\
-1.65\end{array}$ & $\begin{array}{l}1.35 \\
1.34 \\
1.34 \\
1.02 \\
0.97\end{array}$ & $\begin{array}{l}247 \\
250 \\
250 \\
252 \\
255\end{array}$ & $\begin{array}{r}17.98 \\
10.03 \\
5.80 \\
3.25 \\
1.75 \\
0.31\end{array}$ & $\begin{array}{l}291.0 \\
290.09 \\
290.0 \\
290.3 \\
289.7 \\
290.2\end{array}$ \\
\hline
\end{tabular}

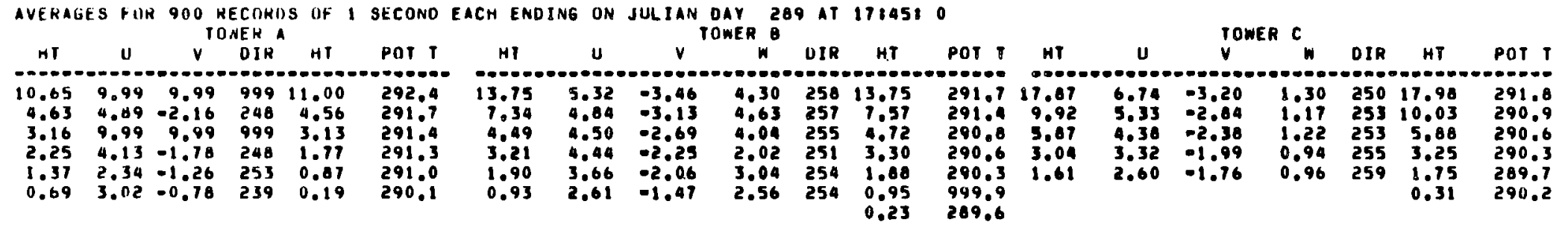

AVERAGES FOR 900 RECORDS OF 1 SECOND EACH ENOING ON JULIAN DAY 209 AT 108020

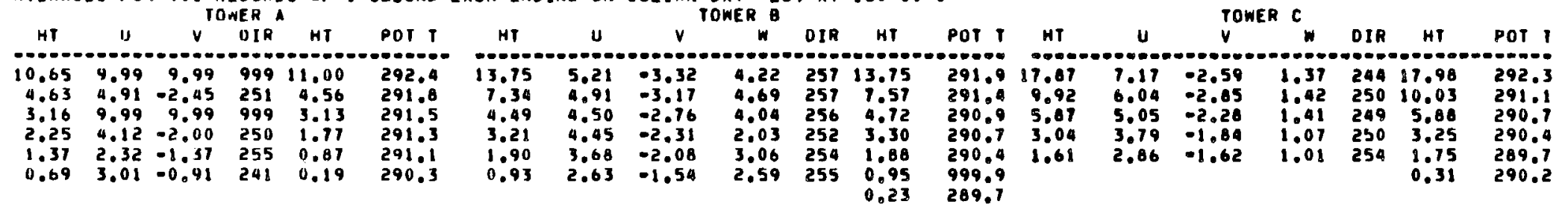


OCTOBER 16, 1981: 1510-2215 PST

\begin{tabular}{|c|c|c|c|c|c|c|c|c|c|c|c|c|c|c|c|c|c|c|}
\hline $\begin{array}{l}\text { AVERAGES } \\
\text { HT }\end{array}$ & $\triangle \mathrm{SFOH}$ & $v^{1000}$ & $\begin{array}{l}\text { IECOHDS } \\
\text { BNER A } \\
\text { DIH }\end{array}$ & $\begin{array}{ll}S \text { OF } 1 \\
\text { HT }\end{array}$ & $\begin{array}{l}\text { SECOND } \\
\text { POT I }\end{array}$ & $\begin{array}{c}\text { EACH ENDINE } \\
\text { HT }\end{array}$ & $N G$ ON & $\begin{array}{c}\text { JULIAN DAY } \\
\text { TOMER B } \\
V\end{array}$ & $\begin{array}{l}9 \mathrm{AT} \\
\text { DIH }\end{array}$ & HT & POT T & HT & U & $v^{10}$ & TOWEN C & DIR & HT & POT T \\
\hline $\begin{array}{l}10.65 \\
4.63 \\
3.16 \\
2.25 \\
1.37 \\
0.69\end{array}$ & $\begin{array}{l}9.99 \\
4.48 \\
9.99 \\
3.80 \\
2.18 \\
2.78\end{array}$ & $\begin{array}{r}9.99 \\
-2.49 \\
9.99 \\
-2.02 \\
-1.36 \\
-0.91\end{array}$ & $\begin{array}{l}999 \\
254 \\
999 \\
252 \\
256 \\
243\end{array}$ & $\begin{array}{l}11.00 \\
4.56 \\
3.13 \\
1.71 \\
0.87 \\
0.19\end{array}$ & $\begin{array}{l}293.3 \\
292.5 \\
292.1 \\
292.0 \\
291.7 \\
290.8\end{array}$ & $\begin{array}{r}13.75 \\
7.34 \\
4.49 \\
3.21 \\
1.90 \\
0.93\end{array}$ & $\begin{array}{l}4.52 \\
4.40 \\
4.17 \\
0.12 \\
3.39 \\
2.47\end{array}$ & $\begin{array}{l}-3.40 \\
-3.117 \\
-2.17 \\
-2.30 \\
-2.04 \\
-1.52\end{array}$ & $\begin{array}{l}261 \\
260 \\
258 \\
254 \\
255 \\
256\end{array}$ & $\begin{array}{r}13.75 \\
7.57 \\
4.72 \\
3.30 \\
1.88 \\
0.95 \\
0.23\end{array}$ & $\begin{array}{l}292.9 \\
292.2 \\
291.6 \\
291.3 \\
290.9 \\
999.9 \\
290.2\end{array}$ & $\begin{array}{r}17.07 \\
9.92 \\
5.87 \\
3.04 \\
1.61\end{array}$ & $\begin{array}{l}4.71 \\
3.90 \\
3.04 \\
2.07 \\
1.50\end{array}$ & $\begin{array}{l}-2.93 \\
-2.63 \\
-2.03 \\
-1.60 \\
-1.33\end{array}$ & $\begin{array}{l}0.98 \\
0.72 \\
0.81 \\
0.56 \\
0.59\end{array}$ & $\begin{array}{l}256 \\
258 \\
258 \\
262 \\
266\end{array}$ & $\begin{array}{l}11.98 \\
10.03 \\
5.88 \\
3.25 \\
1.75 \\
0.31\end{array}$ & $\begin{array}{l}292.9 \\
291.6 \\
291.0 \\
290.7 \\
290.1 \\
290.4\end{array}$ \\
\hline
\end{tabular}

AVERAGES FUR 900 RECOROS OF I SECONO EACH ENDING ON JULIAN DAY 209 AT 1813080

\begin{tabular}{|c|c|c|c|c|c|c|c|c|c|c|c|c|c|c|c|c|c|c|c|}
\hline HT & $u$ & $v$ & OIH & HT & POI T & HT & u & $v$ & $w$ & OIR & $H T$ & & HT & $\mathbf{u}$ & & & DIR & HT & РОT \\
\hline $\begin{array}{l}10.65 \\
4.63 \\
3.16 \\
2.25 \\
1.37 \\
0.69\end{array}$ & $\begin{array}{l}9.99 \\
2.83 \\
9.99 \\
2.59 \\
1.57 \\
2.01\end{array}$ & $\begin{array}{r}9.99 \\
-2.94 \\
9.99 \\
-1.80 \\
-1.25 \\
-0.87\end{array}$ & $\begin{array}{l}999 \\
265 \\
999 \\
261 \\
263 \\
248\end{array}$ & $\begin{array}{r}11.00 \\
4.56 \\
3.13 \\
1.11 \\
0.87 \\
0.19\end{array}$ & $\begin{array}{l}293.8 \\
293.1 \\
292.7 \\
292.4 \\
292.1 \\
290.9\end{array}$ & $\begin{array}{r}13.75 \\
7.34 \\
4.49 \\
3.21 \\
1.90 \\
0.93\end{array}$ & $\begin{array}{l}2.01 \\
2.15 \\
2.27 \\
2.30 \\
2.06 \\
1.46\end{array}$ & $\begin{array}{l}-3.03 \\
-2.67 \\
-2.29 \\
-1.93 \\
-1.174 \\
-1.23\end{array}$ & $\begin{array}{l}1.67 \\
2.26 \\
2.12 \\
1.15 \\
1.70 \\
1.53\end{array}$ & $\begin{array}{l}281 \\
276 \\
270 \\
264 \\
265 \\
265\end{array}$ & $\begin{array}{r}13.75 \\
7.57 \\
4.72 \\
3.30 \\
1.80 \\
0.95\end{array}$ & $\begin{array}{l}293.0 \\
292.6 \\
292.1 \\
291.9 \\
291.4 \\
999.9\end{array}$ & $\begin{array}{r}17.87 \\
9.92 \\
5.87 \\
3.04 \\
1.61\end{array}$ & $\begin{array}{l}0.12 \\
0.22 \\
0.43 \\
0.61 \\
0.74\end{array}$ & $\begin{array}{l}-1.1 \\
-1.7 \\
-1.7 \\
-1.5 \\
-1.3\end{array}$ & $\begin{array}{l}0.21 \\
0.11 \\
0.36 \\
0.22 \\
0.42\end{array}$ & $\begin{array}{l}311 \\
308 \\
301 \\
293 \\
285\end{array}$ & $\begin{array}{r}17.98 \\
10.03 \\
5.80 \\
3.25 \\
1.75 \\
0.31\end{array}$ & $\begin{array}{l}292.0 \\
291.6 \\
291.5 \\
291.1 \\
289.9 \\
288.7\end{array}$ \\
\hline
\end{tabular}

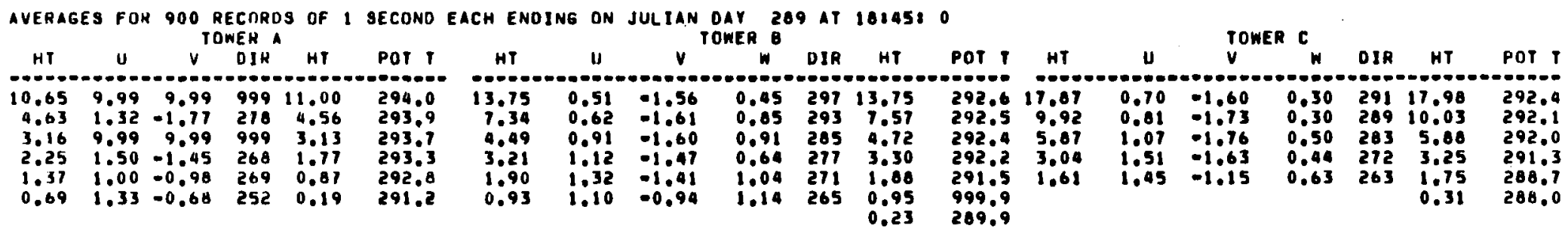

AVERAGES FOR 900 RECORDS OF I SECOND EACH ENOING ON JULIAN OAY 289 AT 191080

\begin{tabular}{|c|c|c|c|c|c|c|c|c|c|c|c|c|c|c|c|c|c|c|c|}
\hline HT & II & $v$ & OIR & HI & POI 1 & HT & u & $v$ & 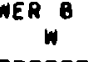 & OIR & HT & Por T & & u & $v^{10}$ & ER $C_{W}$ & OIR & HT & POT \\
\hline $\begin{array}{r}10.65 \\
4.63 \\
3.16 \\
2.25 \\
1.37 \\
0.69\end{array}$ & $\begin{array}{l}9.99 \\
1.66 \\
9.99 \\
1.80 \\
1.21 \\
1.55\end{array}$ & $\begin{array}{r}9.99 \\
-1.20 \\
9.99 \\
-1.01 \\
-0.76 \\
-0.45\end{array}$ & $\begin{array}{l}999 \\
260 \\
999 \\
254 \\
256 \\
241\end{array}$ & $\begin{array}{r}11.00 \\
4.56 \\
3.13 \\
1.71 \\
0.87 \\
0.119\end{array}$ & $\begin{array}{l}294.0 \\
293.9 \\
293.8 \\
293.4 \\
292.8 \\
291.2\end{array}$ & $\begin{array}{r}13.75 \\
7.34 \\
4.49 \\
3.21 \\
1.90 \\
0.93\end{array}$ & $\begin{array}{l}0.35 \\
0.44 \\
0.69 \\
0.81 \\
1.07 \\
0.92\end{array}$ & $\begin{array}{l}-0.077 \\
-0.53 \\
-0.066 \\
-0.68 \\
-0.73 \\
-0.94\end{array}$ & $\begin{array}{l}0.28 \\
0.59 \\
0.68 \\
0.52 \\
0.66 \\
0.91\end{array}$ & $\begin{array}{l}278 \\
275 \\
268 \\
265 \\
259 \\
250\end{array}$ & $\begin{array}{r}13.75 \\
7.57 \\
4.72 \\
3.30 \\
1.88 \\
0.95 \\
0.23\end{array}$ & $\begin{array}{l}292.4 \\
292.4 \\
292.3 \\
292.2 \\
291.6 \\
999.9 \\
289.8\end{array}$ & $\begin{array}{r}17.87 \\
9.92 \\
5.87 \\
3.04 \\
1.61\end{array}$ & $\begin{array}{r}-0.30 \\
-0.13 \\
0.118 \\
0.60 \\
0.66\end{array}$ & $\begin{array}{l}-1.15 \\
-1.59 \\
-1.01 \\
-1.17 \\
-0.05\end{array}$ & $\begin{array}{r}0.16 \\
-0.04 \\
0.19 \\
0.13 \\
0.33\end{array}$ & $\begin{array}{l}324 \\
319 \\
307 \\
287 \\
277\end{array}$ & $\begin{array}{r}17.98 \\
10.03 \\
5.88 \\
3.25 \\
1.05 \\
0.31\end{array}$ & $\begin{array}{l}291.6 \\
291.2 \\
290.9 \\
290.1 \\
288.0 \\
287.3\end{array}$ \\
\hline
\end{tabular}


OCTOBER 16. 1981: $1510-2215$ PST

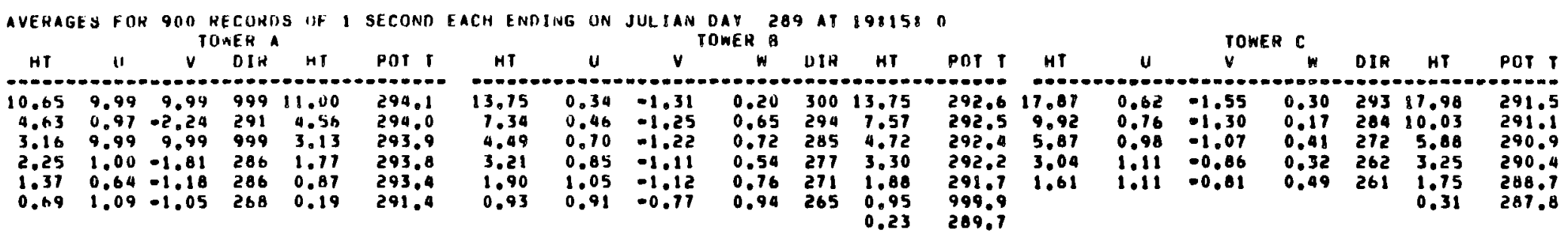

AVERAGES FOR 900 RECORDS OF I SECOND EACH ENOING ON JULIAN DAY 289 AT 1913010

\begin{tabular}{|c|c|c|c|c|c|c|c|c|c|c|c|c|c|c|c|c|c|c|c|}
\hline HT & U & $v$ & DIR & HT & POT T & HT & U & $v$ & $w$ & DIA & HT & Por 1 & HT & U & v & $w$ & DIR & HT & POT $T$ \\
\hline $\begin{array}{r}10.65 \\
9.63 \\
3.16 \\
2.25 \\
1.37 \\
0.69\end{array}$ & $\begin{array}{l}9.99 \\
1.25 \\
9.99 \\
1.22 \\
0.75 \\
1.16\end{array}$ & $\begin{array}{r}9.99 \\
-2.19 \\
9.99 \\
-2.23 \\
-1.48 \\
-1.33\end{array}$ & $\begin{array}{l}999 \\
290 \\
999 \\
286 \\
288 \\
274\end{array}$ & $\begin{array}{r}11.00 \\
4.56 \\
3.11 \\
1.71 \\
0.87 \\
0.19\end{array}$ & $\begin{array}{l}294.4 \\
294.02 \\
294.0 \\
293.9 \\
293.6 \\
292.2\end{array}$ & $\begin{array}{r}13.15 \\
7.34 \\
4.49 \\
3.21 \\
1.90 \\
0.93\end{array}$ & $\begin{array}{l}0.44 \\
0.59 \\
0.18 \\
0.89 \\
0.99 \\
0.18\end{array}$ & $\begin{array}{l}-2.09 \\
-2.54 \\
-2.28 \\
-1.98 \\
-1.75 \\
-1.25\end{array}$ & $\begin{array}{l}0.36 \\
0.89 \\
0.86 \\
0.56 \\
0.84 \\
0.91\end{array}$ & $\begin{array}{l}306 \\
302 \\
296 \\
290 \\
205 \\
202\end{array}$ & $\begin{array}{r}13.75 \\
1.57 \\
0.72 \\
3.30 \\
1.08 \\
0.95 \\
0.23\end{array}$ & $\begin{array}{l}293.4 \\
293.3 \\
293.0 \\
292.8 \\
292.2 \\
999.9 \\
290.8\end{array}$ & $\begin{array}{r}17.87 \\
9.92 \\
5.87 \\
3.04 \\
1.61\end{array}$ & $\begin{array}{l}0.73 \\
0.04 \\
0.93 \\
1.08 \\
1.19\end{array}$ & $\begin{array}{l}2.00 \\
1.94 \\
1.08 \\
0.74 \\
0.43\end{array}$ & $\begin{array}{l}0.32 \\
0.02 \\
0.23 \\
0.22 \\
0.42\end{array}$ & $\begin{array}{l}155 \\
165 \\
175 \\
190 \\
205\end{array}$ & $\begin{array}{r}17.98 \\
10.03 \\
5.88 \\
3.25 \\
1.75 \\
0.31\end{array}$ & $\begin{array}{l}291.7 \\
291.4 \\
291.4 \\
290.7 \\
289.3 \\
281.3\end{array}$ \\
\hline
\end{tabular}

AVERAGES FOR 9OO RECOROS OF I SECOND EACH ENDING ON JULIAM DAY 209 AT 1914510

\begin{tabular}{|c|c|c|c|c|c|c|c|c|c|c|c|c|c|c|c|c|c|c|c|}
\hline HT & $u$ & $v$ & DIR & AT & POT 1 & MT & u & $v$ & $w$ & DIA & HT & Pot & HT & $\mathbf{u}$ & $v^{T 01}$ & $c^{c}$ & OIR & $\mathrm{HT}$ & POT \\
\hline $\begin{array}{r}10.65 \\
4.63 \\
3.16 \\
2.25 \\
1.37 \\
0.69\end{array}$ & $\begin{array}{l}9.99 \\
2.17 \\
9.99 \\
2.25 \\
1.48 \\
1.83\end{array}$ & $\begin{array}{r}9.99 \\
-2.31 \\
9.99 \\
-1.84 \\
-1.22 \\
-0.71\end{array}$ & $\begin{array}{l}999 \\
271 \\
999 \\
264 \\
264 \\
297\end{array}$ & $\begin{array}{r}11.00 \\
4.56 \\
3.13 \\
1.77 \\
0.87 \\
0.19\end{array}$ & $\begin{array}{l}294.5 \\
294.3 \\
293.4 \\
293.3 \\
292.1 \\
291.4\end{array}$ & $\begin{array}{r}13.75 \\
7.34 \\
9.49 \\
3.21 \\
1.90 \\
0.93\end{array}$ & $\begin{array}{l}1.60 \\
1.67 \\
2.11 \\
2.44 \\
2.23 \\
1.54\end{array}$ & $\begin{array}{l}-2.37 \\
-2.42 \\
-2.32 \\
-1.96 \\
-1.06 \\
-1.08\end{array}$ & $\begin{array}{l}1.34 \\
1.82 \\
1.92 \\
1.19 \\
1.81 \\
1.57\end{array}$ & $\begin{array}{l}280 \\
280 \\
272 \\
263 \\
261 \\
260\end{array}$ & $\begin{array}{r}13.75 \\
7.57 \\
4.72 \\
3.30 \\
1.08 \\
0.95 \\
0.23\end{array}$ & $\begin{array}{l}293.1 \\
293.6 \\
293.1 \\
292.5 \\
291.6 \\
999.9 \\
290.3\end{array}$ & $\begin{array}{r}17.87 \\
9.92 \\
5.87 \\
3.04 \\
1.61\end{array}$ & $\begin{array}{l}0.47 \\
0.52 \\
0.67 \\
0.74 \\
0.88\end{array}$ & $\begin{array}{r}0.57 \\
0.14 \\
0.03 \\
-0.12 \\
-0.27\end{array}$ & $\begin{array}{l}0.26 \\
0.11 \\
0.25 \\
0.19 \\
0.37\end{array}$ & $\begin{array}{l}174 \\
209 \\
222 \\
234 \\
241\end{array}$ & $\begin{array}{r}17.98 \\
10.03 \\
5.80 \\
3.25 \\
1.75 \\
0.31\end{array}$ & $\begin{array}{l}291.9 \\
291.7 \\
291.4 \\
290.9 \\
289.7 \\
287.9\end{array}$ \\
\hline
\end{tabular}

AVERAGES FOK 900 RECOROS OF 1 SECOND EACH ENDING ON JULIAN DAY 289 AT 201080

\begin{tabular}{|c|c|c|c|c|c|c|c|c|c|c|c|c|c|c|c|c|c|c|c|}
\hline HT & 11 & $v$ & OIK & HT & POT T & HT & u & $v$ & $\omega$ & DIR & HT & POT & HT & u & $v$ & $w$ & OIR & $\mathrm{HT}$ & POT $T$ \\
\hline $\begin{array}{l}0.65 \\
4.63 \\
3.16 \\
2.25 \\
1.37 \\
0.69\end{array}$ & $\begin{array}{l}9.99 \\
3.08 \\
9.99 \\
2.65 \\
1.70 \\
2.00\end{array}$ & $\begin{array}{r}9.99 \\
-1.28 \\
9.99 \\
-1.04 \\
-0.84 \\
-0.040\end{array}$ & $\begin{array}{l}999 \\
247 \\
999 \\
246 \\
251 \\
236\end{array}$ & $\begin{array}{r}11.00 \\
4.56 \\
3.13 \\
1.77 \\
0.87 \\
0.19\end{array}$ & $\begin{array}{l}293.9 \\
293.2 \\
292.9 \\
292.7 \\
292.3 \\
291.1\end{array}$ & $\begin{array}{r}13.75 \\
7.34 \\
4.49 \\
3.21 \\
1.90 \\
0.93\end{array}$ & $\begin{array}{l}2.75 \\
2.66 \\
2.79 \\
2.91 \\
2.51 \\
1.74\end{array}$ & $\begin{array}{l}-2.23 \\
-1.86 \\
-1.61 \\
-1.33 \\
-1.20 \\
-0.74\end{array}$ & $\begin{array}{l}2.21 \\
2.64 \\
2.51 \\
1.37 \\
2.03 \\
1.73\end{array}$ & $\begin{array}{l}263 \\
259 \\
255 \\
249 \\
250 \\
248\end{array}$ & $\begin{array}{r}13.75 \\
7.57 \\
4.72 \\
3.330 \\
1.80 \\
0.95 \\
0.23\end{array}$ & $\begin{array}{l}293.4 \\
292.8 \\
292.2 \\
291.9 \\
291.2 \\
999.9 \\
290.1\end{array}$ & $\begin{array}{r}17.87 \\
9.92 \\
5.87 \\
3.04 \\
1.61\end{array}$ & $\begin{array}{l}2.46 \\
2.27 \\
2.03 \\
1.65 \\
1.43\end{array}$ & $\begin{array}{l}-0.64 \\
=0.36 \\
=0.25 \\
-0.20 \\
=0.21\end{array}$ & $\begin{array}{l}0.59 \\
0.44 \\
0.64 \\
0.46 \\
0.53\end{array}$ & $\begin{array}{l}239 \\
234 \\
232 \\
231 \\
233\end{array}$ & $\begin{array}{r}17.98 \\
10.03 \\
5.80 \\
3.25 \\
1.75 \\
0.31\end{array}$ & $\begin{array}{l}292.4 \\
291.7 \\
291.4 \\
290.9 \\
290.0 \\
288.7\end{array}$ \\
\hline
\end{tabular}


OCTOBER 16, 1981: 1510-2215 PST

\begin{tabular}{|c|c|c|c|c|c|c|c|c|c|c|c|c|c|c|c|c|c|c|c|}
\hline $\begin{array}{c}\text { A VERAGES } \\
\text { HT }\end{array}$ & S FOR & 900 & $\begin{array}{l}\text { ECORDS } \\
\text { WERAA } \\
\text { DIRA }\end{array}$ & 8 of 1 & SECOND & EACH ENDING & IG ON & JULIAN & $\begin{array}{c}\text { DAY } \\
\text { TOWFR } \\
\end{array}$ & 89 & $\begin{array}{c}20: 151 \\
\text { HT }\end{array}$ & POT & HT & $\mathbf{v}$ & $v^{T 0}$ & 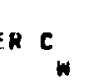 & DIK & HT & POT \\
\hline $\begin{array}{r}10.65 \\
4.63 \\
3.16 \\
2.25 \\
1.37 \\
0.69\end{array}$ & $\begin{array}{l}9.99 \\
3.49 \\
9.99 \\
3.03 \\
1.87 \\
2.24\end{array}$ & $\begin{array}{r}9.99 \\
-2.04 \\
9.99 \\
-1.61 \\
-1.16 \\
-0.16\end{array}$ & $\begin{array}{l}9991 \\
255 \\
999 \\
252 \\
256 \\
243\end{array}$ & $\begin{array}{r}11.00 \\
4.56 \\
3.13 \\
1.77 \\
0.87 \\
0.19\end{array}$ & $\begin{array}{l}293.6 \\
292.6 \\
292.6 \\
292.4 \\
292.1 \\
291.1\end{array}$ & $\begin{array}{r}13.75 \\
7.34 \\
4.49 \\
3.21 \\
1.90 \\
0.93\end{array}$ & $\begin{array}{l}3.26 \\
3.06 \\
3.02 \\
3.11 \\
2.62 \\
1.80\end{array}$ & $\begin{array}{l}-3.20 \\
-2.448 \\
-2.12 \\
-1.75 \\
-1.58 \\
-1.110\end{array}$ & $\begin{array}{l}2.64 \\
3.05 \\
2.77 \\
1.44 \\
2.17 \\
1.88\end{array}$ & $\begin{array}{l}269 \\
264 \\
260 \\
254 \\
256 \\
255\end{array}$ & $\begin{array}{r}13.75 \\
7.57 \\
4.72 \\
3.30 \\
1.88 \\
0.95 \\
0.23\end{array}$ & $\begin{array}{l}293.0 \\
292.5 \\
292.0 \\
291.7 \\
291.2 \\
999.9 \\
290.2\end{array}$ & $\begin{array}{r}17.87 \\
9.92 \\
5.87 \\
3.04 \\
1.61\end{array}$ & $\begin{array}{l}3.37 \\
3.20 \\
2.97 \\
2.26 \\
1.77\end{array}$ & $\begin{array}{l}-2.23 \\
-2.02 \\
-1.70 \\
-1.36 \\
-1.15\end{array}$ & $\begin{array}{l}0.78 \\
0.76 \\
0.92 \\
0.63 \\
0.68\end{array}$ & $\begin{array}{l}258 \\
257 \\
254 \\
256 \\
258\end{array}$ & $\begin{array}{r}17.98 \\
10.03 \\
5.08 \\
3.25 \\
1.75 \\
0.31\end{array}$ & $\begin{array}{l}292.7 \\
292.7 \\
291.5 \\
290.7 \\
289.9 \\
289.9\end{array}$ \\
\hline
\end{tabular}

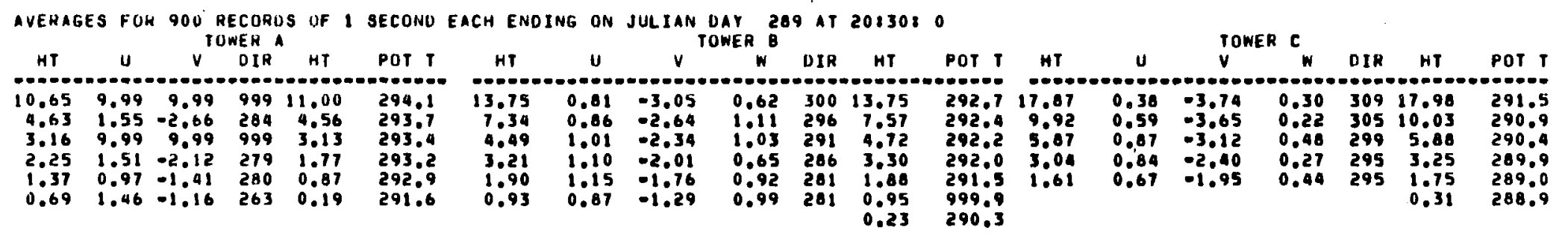

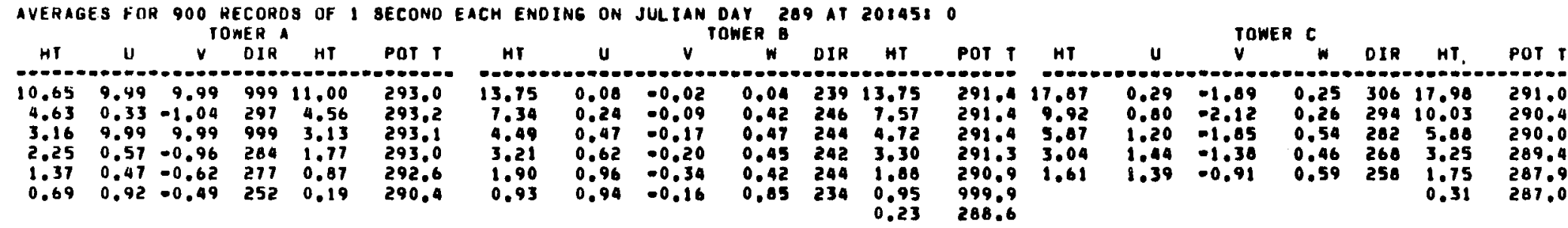

AVERAGES FON 900 RECORDS OF 1 BECONO EACH ENDING ON JULIAN DAY 209 AT 211080

\begin{tabular}{|c|c|c|c|c|c|c|c|c|c|c|c|c|c|c|c|c|c|c|c|}
\hline HT & 11 & $v$ & OIR & HT & POT 1 & HT & u & $v$ & H & DIR & HT & POT & HT & U & $v$ & $\boldsymbol{w}$ & OIR & HT & POT T \\
\hline $\begin{array}{r}10.65 \\
4.63 \\
3.16 \\
2.25 \\
1.37 \\
0.69\end{array}$ & $\begin{array}{l}9.99 \\
0.48 \\
9.99 \\
0.65 \\
0.45 \\
0.90\end{array}$ & $\begin{array}{r}9.99 \\
-2.08 \\
9.99 \\
-1.79 \\
-1.12 \\
-1.06\end{array}$ & $\begin{array}{l}999 \\
301 \\
999 \\
295 \\
292 \\
274\end{array}$ & $\begin{array}{r}11.00 \\
4.56 \\
3.13 \\
1.77 \\
0.87 \\
0.19\end{array}$ & $\begin{array}{l}293.1 \\
293.1 \\
293.0 \\
292.8 \\
292.1 \\
290.6\end{array}$ & $\begin{array}{r}13.75 \\
7.34 \\
4.49 \\
3.21 \\
1.90 \\
0.93\end{array}$ & $\begin{array}{l}0.25 \\
0.29 \\
0.54 \\
0.70 \\
0.99 \\
0.96\end{array}$ & $\begin{array}{l}-0.91 \\
-1.020 \\
-1.037 \\
-1.034 \\
-1.033 \\
-0.94\end{array}$ & $\begin{array}{l}0.30 \\
0.60 \\
0.63 \\
0.49 \\
0.73 \\
0.98\end{array}$ & $\begin{array}{l}299 \\
301 \\
293 \\
287 \\
278 \\
269\end{array}$ & $\begin{array}{r}13.75 \\
7.57 \\
4.72 \\
3.30 \\
1.80 \\
0.95 \\
0.23\end{array}$ & $\begin{array}{l}291.3 \\
291.3 \\
291.3 \\
291.1 \\
290.6 \\
999.9 \\
288.7\end{array}$ & $\begin{array}{r}17.87 \\
9.92 \\
5.07 \\
3.04 \\
1.61\end{array}$ & $\begin{array}{l}0.26 \\
0.57 \\
0.94 \\
1.38 \\
1.55\end{array}$ & $\begin{array}{l}-1.34 \\
-1.65 \\
-1.61 \\
-1.46 \\
-1.08\end{array}$ & $\begin{array}{l}0.23 \\
0.26 \\
0.46 \\
0.40 \\
0.63\end{array}$ & $\begin{array}{l}303 \\
296 \\
284 \\
271 \\
259\end{array}$ & $\begin{array}{r}17.98 \\
10.03 \\
5.08 \\
3.25 \\
1.75 \\
0.31\end{array}$ & $\begin{array}{l}291.0 \\
290.0 \\
290.4 \\
289.8 \\
287.2 \\
286.1\end{array}$ \\
\hline
\end{tabular}


OCTOBER 16, 1981: 1510-2215 PST

AVERAGES FOR 9OO RECORDS OF I SECOND EACH ENDING ON JULIAN DAY 284 AT 2181580

\begin{tabular}{|c|c|c|c|c|c|c|c|c|c|c|c|c|c|c|c|c|c|c|c|}
\hline HT & 11 & $v$ & OIR & HT & POI 1 & $\mathrm{HT}$ & u & $v$ & $w$ & DIR & $\mathrm{HT}$ & Por 1 & HT & u & $v$ & $w$ & DIR & HT & POT T \\
\hline $\begin{array}{r}10.65 \\
4.63 \\
3.16 \\
2.25 \\
1.37 \\
0.69\end{array}$ & $\begin{array}{l}9.99 \\
0.45 \\
9.99 \\
0.72 \\
0.61 \\
1.09\end{array}$ & $\begin{array}{r}9.99 \\
-1.17 \\
9.99 \\
-1.09 \\
-0.117 \\
-0.55\end{array}$ & $\begin{array}{l}999 \\
294 \\
999 \\
281 \\
276 \\
251\end{array}$ & $\begin{array}{r}11.01 \\
4.56 \\
3.13 \\
1.71 \\
0.87 \\
0.19\end{array}$ & $\begin{array}{l}292.7 \\
292.8 \\
292.8 \\
292.6 \\
292.0 \\
289.1\end{array}$ & $\begin{array}{r}13.75 \\
7.34 \\
4.49 \\
3.21 \\
1.99 \\
0.93\end{array}$ & $\begin{array}{l}0.29 \\
0.35 \\
0.64 \\
0.86 \\
1.21 \\
1.18\end{array}$ & $\begin{array}{l}-0.53 \\
-0.64 \\
-0.75 \\
-0.77 \\
-0.06 \\
-0.52\end{array}$ & $\begin{array}{l}0.31 \\
0.59 \\
0.66 \\
0.55 \\
0.82 \\
1.12\end{array}$ & $\begin{array}{l}285 \\
286 \\
274 \\
266 \\
260 \\
298\end{array}$ & $\begin{array}{r}13.75 \\
7.51 \\
4.72 \\
3.30 \\
1.08 \\
0.95 \\
0.23\end{array}$ & $\begin{array}{l}291.4 \\
291.5 \\
291.5 \\
291.4 \\
290.7 \\
999.9 \\
288.3\end{array}$ & $\begin{array}{r}17.67 \\
9.92 \\
5.87 \\
3.04 \\
1.61\end{array}$ & $\begin{array}{l}0.41 \\
0.61 \\
1.12 \\
1.75 \\
1.77\end{array}$ & $\begin{array}{l}-0.25 \\
-0.38 \\
-0.25 \\
-0.15 \\
-0.15\end{array}$ & $\begin{array}{l}0.24 \\
0.23 \\
0.46 \\
0.47 \\
0.63\end{array}$ & $\begin{array}{l}256 \\
257 \\
237 \\
229 \\
229\end{array}$ & $\begin{array}{r}17.98 \\
10.03 \\
5.88 \\
3.25 \\
1.75 \\
0.31\end{array}$ & $\begin{array}{l}290.8 \\
290.6 \\
290.4 \\
289.4 \\
286.7 \\
285.8\end{array}$ \\
\hline
\end{tabular}

AVERAGES FUR 9UO RECORDS OF 1 SECOND EACH ENDING ON JULIAN DAY 289 AT 2113010

\begin{tabular}{|c|c|c|c|c|c|c|c|c|c|c|c|c|c|c|c|c|c|c|c|}
\hline \multirow{2}{*}{\multicolumn{7}{|c|}{$y^{\text {TOWER }}$ OIR $^{A}$}} & \multirow{2}{*}{\multicolumn{5}{|c|}{ TOWER B }} & \multicolumn{8}{|c|}{ TOWER C } \\
\hline & & & & & & & & & & & & Pot T & MT & u & & & DIR & HT & POT \\
\hline $\begin{array}{l}10.65 \\
4.63 \\
3.16 \\
2.25 \\
1.37 \\
0.69\end{array}$ & $\begin{array}{l}9.99 \\
0.53 \\
9.99 \\
0.01 \\
0.56 \\
1.10\end{array}$ & $\begin{array}{r}9.99 \\
-0.49 \\
9.99 \\
-0.38 \\
-0.34 \\
-0.11\end{array}$ & $\begin{array}{l}999 \\
267 \\
999 \\
249 \\
256 \\
233\end{array}$ & $\begin{array}{r}11.00 \\
4.56 \\
3.13 \\
1.77 \\
0.87 \\
0.19\end{array}$ & $\begin{array}{l}292.6 \\
292.7 \\
292.7 \\
292.6 \\
292.1 \\
289.1\end{array}$ & $\begin{array}{r}13.75 \\
7.34 \\
4.49 \\
3.21 \\
1.90 \\
0.93\end{array}$ & $\begin{array}{l}0.36 \\
0.41 \\
0.76 \\
0.94 \\
1.27 \\
1.31\end{array}$ & $\begin{array}{r}0.46 \\
0.30 \\
0.09 \\
0.02 \\
-0.27 \\
-0.14\end{array}$ & $\begin{array}{l}0.33 \\
0.55 \\
0.70 \\
0.50 \\
0.80 \\
1.14\end{array}$ & $\begin{array}{l}172 \\
109 \\
217 \\
223 \\
236 \\
231\end{array}$ & $\begin{array}{r}13.75 \\
7.57 \\
1.72 \\
3.30 \\
1.80 \\
0.95 \\
0.23\end{array}$ & $\begin{array}{l}291.3 \\
291.4 \\
291.4 \\
291.4 \\
290.0 \\
999.9 \\
287.8\end{array}$ & $\begin{array}{r}17.87 \\
9.92 \\
5.87 \\
3.04 \\
1.61\end{array}$ & $\begin{array}{l}0.31 \\
0.57 \\
0.94 \\
1.30 \\
1.73\end{array}$ & $\begin{array}{r}0.79 \\
0.51 \\
0.10 \\
0.15 \\
-0.08\end{array}$ & $\begin{array}{l}0.23 \\
0.25 \\
0.41 \\
0.36 \\
0.59\end{array}$ & $\begin{array}{l}156 \\
183 \\
202 \\
218 \\
227\end{array}$ & $\begin{array}{r}17.98 \\
10.03 \\
5.08 \\
3.25 \\
1.75 \\
0.31\end{array}$ & $\begin{array}{l}290.5 \\
290.2 \\
290.2 \\
289.7 \\
287.3 \\
285.1\end{array}$ \\
\hline
\end{tabular}

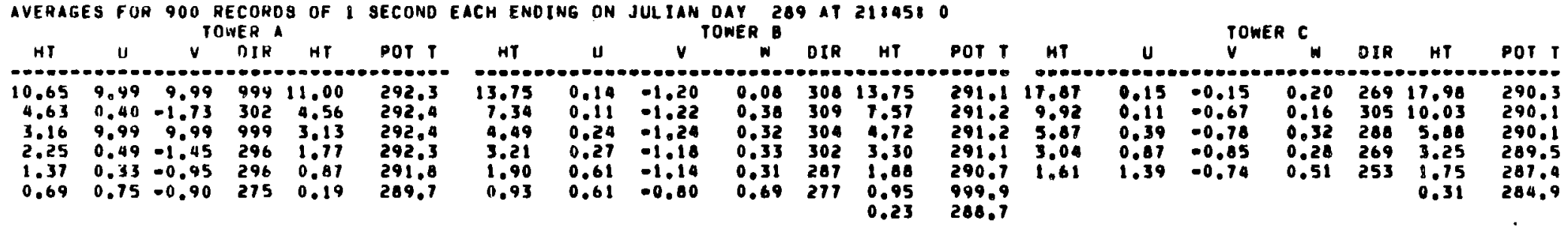

AVERAGES FOR 900 RECORDS OF 1 SECOND EACH ENOING ON JULIAN OAY 289 AT 221 O2 0

\begin{tabular}{|c|c|c|c|c|c|c|c|c|c|c|c|c|c|c|c|c|c|c|c|}
\hline $\mathrm{HT}$ & u & $v$ & OIR & HT & POT $T$ & HT & U & $v$ & w & DIR & HT & POT 7 & HT & $u$ & $v$ & $w$ & DIR & $H 7$ & POT T \\
\hline $\begin{array}{l}10.65 \\
4.63 \\
3.16 \\
2.25 \\
1.37 \\
0.69\end{array}$ & $\begin{array}{l}9.99 \\
0.99 \\
9.99 \\
1.21 \\
0.74 \\
1.26\end{array}$ & $\begin{array}{r}9.99 \\
-2.44 \\
9.99 \\
-1.89 \\
-1.32 \\
-0.99\end{array}$ & $\begin{array}{l}999 \\
292 \\
999 \\
282 \\
285 \\
263\end{array}$ & $\begin{array}{r}11.00 \\
4.56 \\
3.13 \\
1.77 \\
0.87 \\
0.19\end{array}$ & $\begin{array}{l}292.0 \\
291.9 \\
291.6 \\
291.4 \\
290.9 \\
289.4\end{array}$ & $\begin{array}{r}13.15 \\
7.34 \\
4.49 \\
3.21 \\
1.90 \\
0.93\end{array}$ & $\begin{array}{l}0.67 \\
0.82 \\
1.18 \\
1.46 \\
1.53 \\
1.19\end{array}$ & $\begin{array}{l}-2.55 \\
-2.45 \\
-2.26 \\
-1.95 \\
-1.69 \\
-1.14\end{array}$ & $\begin{array}{l}0.66 \\
1.13 \\
1.19 \\
0.79 \\
1.28 \\
1.26\end{array}$ & $\begin{array}{l}300 \\
296 \\
287 \\
278 \\
272 \\
268\end{array}$ & $\begin{array}{r}13.75 \\
7.57 \\
4.72 \\
3.30 \\
1.80 \\
0.95 \\
0.23\end{array}$ & $\begin{array}{l}291.1 \\
291.0 \\
290.0 \\
290.5 \\
289.8 \\
999.9 \\
288.3\end{array}$ & $\begin{array}{r}17.87 \\
9.92 \\
5.87 \\
3.04 \\
1.61\end{array}$ & $\begin{array}{l}0.89 \\
1.22 \\
1.58 \\
1.60 \\
1.43\end{array}$ & $\begin{array}{l}-2.39 \\
-2.29 \\
-2.09 \\
-1.42 \\
-1.00\end{array}$ & $\begin{array}{l}0.35 \\
0.33 \\
0.63 \\
0.53 \\
0.59\end{array}$ & $\begin{array}{l}294 \\
286 \\
277 \\
266 \\
259\end{array}$ & $\begin{array}{r}17.98 \\
10.03 \\
5.80 \\
3.25 \\
1.75 \\
0.31\end{array}$ & $\begin{array}{l}290.7 \\
290.3 \\
289.9 \\
208.9 \\
287.1 \\
285.9\end{array}$ \\
\hline
\end{tabular}


OCTOBER 16, 1981; 1510-2215 PST

\begin{tabular}{|c|c|c|c|c|c|c|c|c|c|c|c|c|c|c|c|c|c|c|c|}
\hline AVERAGE & $E S F O K$ & 000 & $\begin{array}{l}\text { ECURI } \\
\text { VER }\end{array}$ & I) 0 & $E O N$ & EACH & U 6 & JULIh & $\begin{array}{l}\text { nAY } \\
\text { OAER }\end{array}$ & & $\mu$ & & & & TOWES & & & & \\
\hline HT & $u$ & $v$ & DIH & HI & POT & HT & u & $v$ & W & DIR & HT & POT T & HT & U & $v$ & $\ldots$ & DIR & HT & POT T \\
\hline $\begin{array}{r}10.65 \\
4.63 \\
3.16 \\
2.25 \\
1.37 \\
0.69\end{array}$ & $\begin{array}{l}9.99 \\
1.019 \\
9.99 \\
0.98 \\
0.45 \\
1.07\end{array}$ & $\begin{array}{r}9.49 \\
-3.18 \\
9.99 \\
-2.62 \\
-1.78 \\
-1.62\end{array}$ & $\begin{array}{l}999 \\
296 \\
999 \\
294 \\
300 \\
281\end{array}$ & $\begin{array}{r}11.000 \\
4.56 \\
3.13 \\
1.77 \\
0.87 \\
0.19\end{array}$ & $\begin{array}{l}292.3 \\
292.1 \\
292.0 \\
291.0 \\
291.0 \\
290.0\end{array}$ & $\begin{array}{r}13.75 \\
7.34 \\
4.49 \\
3.21 \\
1.90 \\
0.93\end{array}$ & $\begin{array}{l}1.49 \\
0.57 \\
0.79 \\
0.94 \\
1.01 \\
0.81\end{array}$ & $\begin{array}{l}-2.96 \\
-2.74 \\
-2.54 \\
-2.22 \\
-1.86 \\
-1.35\end{array}$ & $\begin{array}{l}0.44 \\
0.92 \\
0.88 \\
0.59 \\
0.90 \\
0.98\end{array}$ & $\begin{array}{l}305 \\
303 \\
297 \\
292 \\
206 \\
283\end{array}$ & $\begin{array}{r}13.75 \\
7.57 \\
4.72 \\
3.30 \\
1.80 \\
0.95 \\
0.23\end{array}$ & $\begin{array}{l}291.1 \\
291.1 \\
290.9 \\
290.6 \\
290.0 \\
999.9 \\
280.6\end{array}$ & $\begin{array}{r}17.07 \\
9.92 \\
5.67 \\
3.04 \\
1.61\end{array}$ & $\begin{array}{l}0.58 \\
0.87 \\
1.33 \\
1.68 \\
1.42\end{array}$ & $\begin{array}{l}-2.43 \\
-2.43 \\
-2.33 \\
-1.81 \\
-1.26\end{array}$ & $\begin{array}{l}0.29 \\
0.25 \\
0.58 \\
0.53 \\
0.62\end{array}$ & $\begin{array}{l}301 \\
295 \\
285 \\
272 \\
266\end{array}$ & $\begin{array}{r}17.98 \\
10.03 \\
5.80 \\
3.25 \\
1.75 \\
0.31\end{array}$ & $\begin{array}{l}290.7 \\
290.4 \\
290.0 \\
280.8 \\
286.9 \\
286.3\end{array}$ \\
\hline
\end{tabular}


L. Tethersonde Data

Data from 24 Tethersonde flights during the four cases are listed below, labeled with the case date and the laugh and landing times. Data are often included after landing of the sonde to give a good sample of the surface temperature and pressure. The Tethersonde data were recorded every 10-20 seconds, but have been edited for this data volume. Below a belght of $50 m$ all data are 11sted. Above $50 \mathrm{~m}$, data samples from successive helghts differing by less than $10 \mathrm{~m}$ have been deleted.

The data colums are time, pressure, helght, temperature, wet bulb temperature, relative humidity, mixing ratio, wind direction, wind speed, and potential temperature. Several flights 11 st 0.0 in the wet bulb temperature column. Although not included in the listing, the wet bulb depression was measured and used to calculate relative humidity and mixing rat10. During the p1rst plight on June 19, 1980 the wet bulb wick went dry around 2310. This is obvious in the data. On some soundings the sonde battery became too weak and data are only listed for a partial flight, e.8. July 2-3, $1981 ; 0123-0140$

On the evening of July 2, 1981 a limited comparison was made between the thermistor carried by the Tethersonde and the aspirated thermistors on the towers. The sonde was placed next to a thermistor at tower B and one at tower C for approximately 5 minutes each, and the indicated temperatures were compared. For these two cases the sonde temperatures averaged about 0.6 degrees higher than the tower temperatures. Hence, caution should be exercised in attempting to match or join Tethersonde temperature profiles with tower profiles. 
June $19-20,1980: 2300-2352$ RST

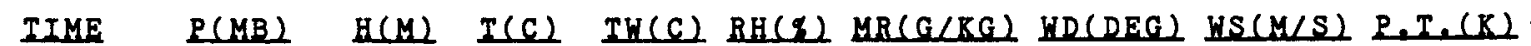

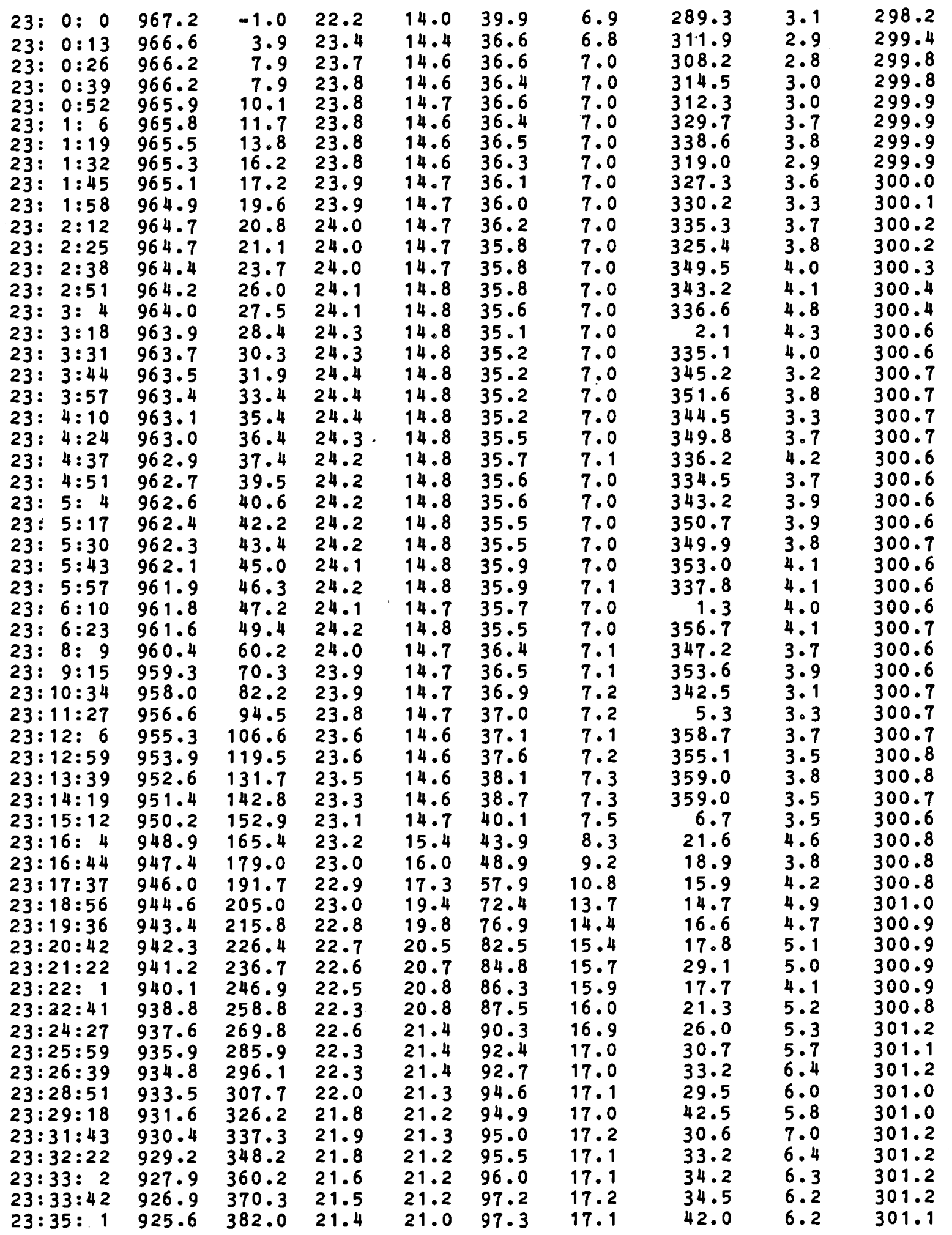




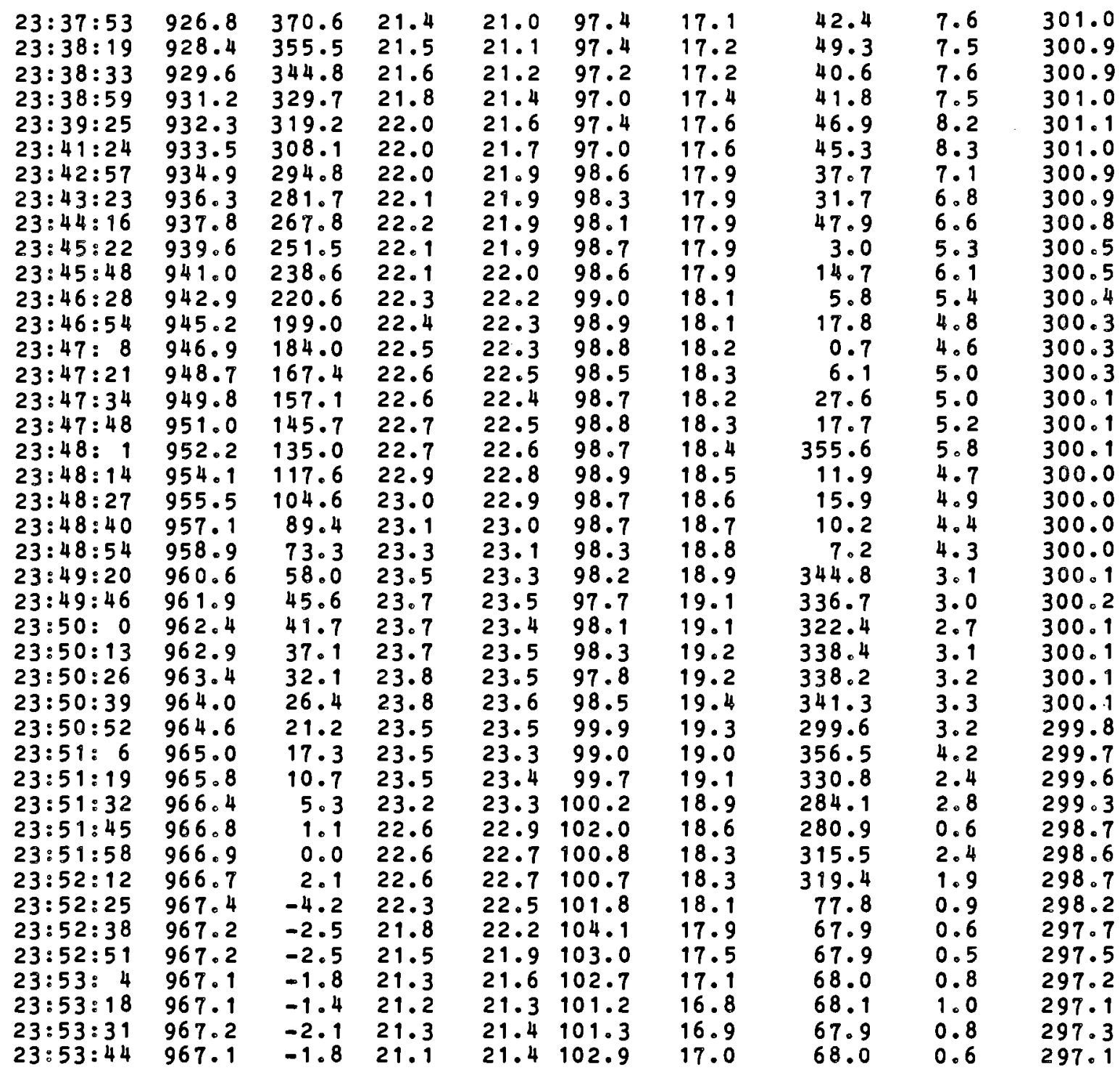

June 19-20, 1980: 0001-0042 PST

IIME $P(M B) \quad H(M) \quad I(C)$ IN(C) RH(S) MR(G/KG) WD(DEG) $N S(M / S)$ P.T. (K)

$\begin{array}{llrlllllll}0: 0: 47 & 966.4 & 3.6 & 21.8 & 13.7 & 40.2 & 6.8 & 293.7 & 2.0 & 297.8 \\ 0: 1: 0 & 966.1 & 5.9 & 21.7 & 13.7 & 40.1 & 6.8 & 286.2 & 2.6 & 297.8 \\ 0: 1: 13 & 965.7 & 9.8 & 22.3 & 13.7 & 37.4 & 6.5 & 295.1 & 3.1 & 298.4 \\ 0: 1: 27 & 965.3 & 13.6 & 22.9 & 13.8 & 35.8 & 6.5 & 332.1 & 3.1 & 299.0 . \\ 0: 1: 40 & 964.8 & 17.5 & 23.0 & 13.8 & 35.0 & 6.4 & 326.8 & 2.9 & 299.2 \\ 0: 1: 53 & 964.3 & 22.0 & 23.2 & 13.9 & 35.0 & 6.5 & 334.5 & 3.2 & 299.5 \\ 0: 2: 7 & 964.0 & 25.4 & 23.3 & 13.9 & 34.7 & 6.5 & 332.5 & 3.2 & 299.5 \\ 0: 2: 20 & 963.6 & 29.2 & 23.3 & 14.0 & 34.8 & 6.5 & 339.6 & 3.2 & 299.7 \\ 0: 2: 33 & 963.2 & 32.8 & 23.4 & 14.0 & 34.4 & 6.4 & 344.1 & 4.1 & 299.7 \\ 0: 2: 46 & 962.8 & 36.4 & 23.5 & 14.1 & 34.5 & 6.5 & 10.6 & 3.2 & 299.9 \\ 0: 3: 0 & 962.3 & 40.4 & 23.5 & 14.1 & 34.8 & 6.6 & 15.2 & 3.2 & 299.9 \\ 0: 3: 13 & 961.8 & 45.2 & 23.5 & 14.1 & 35.0 & 6.6 & 7.1 & 2.8 & 300.0 \\ 0: 3: 26 & 961.4 & 48.7 & 23.4 & 14.1 & 34.8 & 6.6 & 16.8 & 3.1 & 299.9 \\ 0: 4: 6 & 960.2 & 59.4 & 23.4 & 14.0 & 34.6 & 6.5 & 344.6 & 4.0 & 300.1 \\ 0: 4: 45 & 958.9 & 71.0 & 23.4 & 14.0 & 34.7 & 6.5 & 4.9 & 3.7 & 300.1\end{array}$




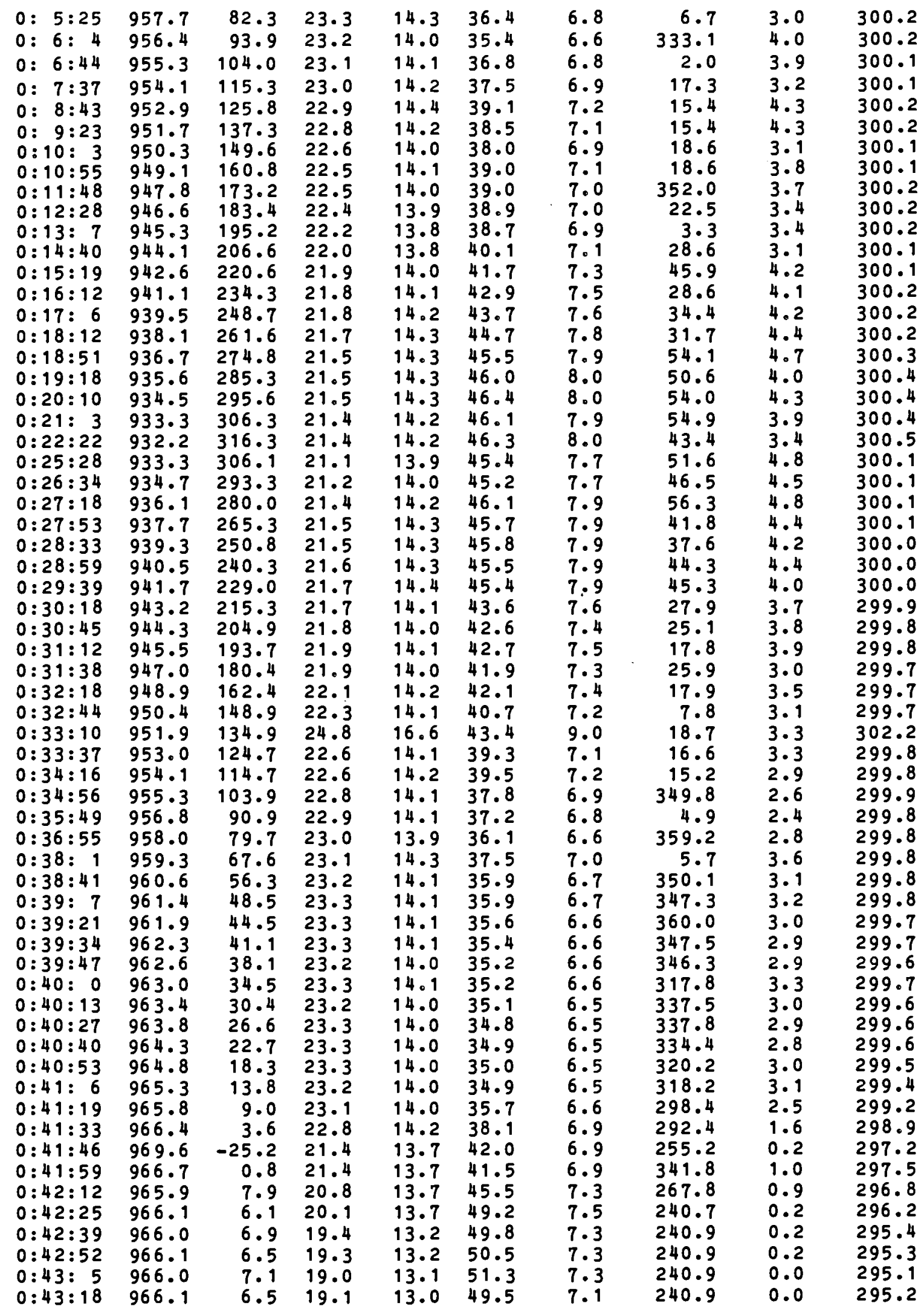




$\begin{array}{llllllllll}0: 43: 31 & 966.1 & 6.5 & 19.1 & 13.0 & 50.4 & 7.2 & 240.8 & 0.2 & 295.1 \\ 0: 43: 45 & 966.1 & 6.6 & 19.1 & 12.9 & 49.0 & 7.1 & 240.8 & 0.3 & 295.2 \\ 0: 43: 58 & 966.0 & 6.8 & 19.1 & 12.9 & 49.2 & 7.1 & 241.0 & 0.3 & 295.2\end{array}$

June 19-20, 1980: 0050-0131 PST

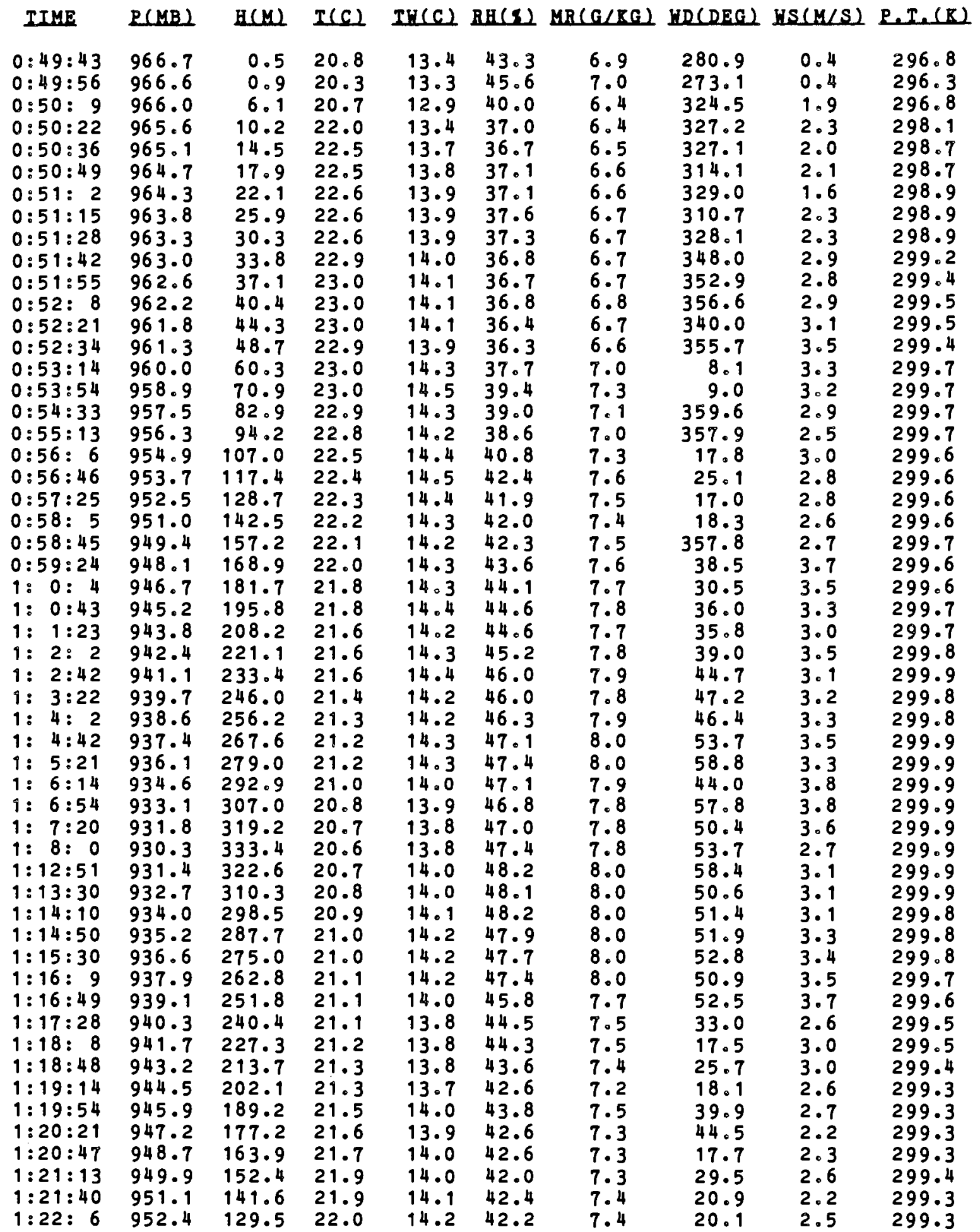




\begin{tabular}{|c|c|c|c|c|c|c|c|c|c|}
\hline $\begin{array}{l}1: 22: 46 \\
1: 23: 12\end{array}$ & $\begin{array}{l}954.1 \\
955.3\end{array}$ & $\begin{array}{l}114.2 \\
103.4\end{array}$ & $\begin{array}{l}22.2 \\
22.1\end{array}$ & $\begin{array}{l}14.1 \\
14.1\end{array}$ & $\begin{array}{l}41.2 \\
41.0\end{array}$ & $\begin{array}{l}7.3 \\
7.2\end{array}$ & $\begin{array}{r}17.1 \\
343.3\end{array}$ & $\begin{array}{l}2.1 \\
2.3\end{array}$ & $\begin{array}{l}299.3 \\
299.1\end{array}$ \\
\hline $1: 23: 52$ & 956.5 & 92.7 & 22.4 & 14.0 & 39.2 & 7.0 & 356.1 & 2.9 & 299.3 \\
\hline $1: 24: 45$ & 957.6 & 82.3 & 22.5 & 14.2 & 39.5 & 7.1 & 359.2 & 2.4 & 299.3 \\
\hline $1: 25: 38$ & 958.9 & 70.5 & 22.5 & 14.3 & 40.4 & 7.2 & 6.7 & 2.3 & 299.3 \\
\hline $1: 26: 31$ & 960.2 & 58.7 & 22.7 & 14.4 & 40.4 & $7 \cdot 3$ & 344.8 & 2.5 & 299.3 \\
\hline $\begin{array}{l}1: 27: 24 \\
1: 27: 37\end{array}$ & 961.4 & $\begin{array}{l}48.3 \\
45.8\end{array}$ & $\begin{array}{l}22.8 \\
22.8\end{array}$ & $\begin{array}{l}14 \cdot 3 \\
14.4\end{array}$ & $\begin{array}{l}39.2 \\
39.4\end{array}$ & $7 \cdot 1$ & $\begin{array}{l}351.8 \\
350.6\end{array}$ & $2 \cdot 3$ & $\begin{array}{l}299.3 \\
299.3\end{array}$ \\
\hline $1: 27: 50$ & 961.9 & 43.2 & 22.8 & 14.3 & 39.0 & 7.1 & 343.4 & 2.2 & 299.3 \\
\hline $1: 28: 3$ & 962.1 & 42.0 & 22.8 & $14 \cdot 3$ & 38.6 & 7.0 & 337.1 & 2.4 & $9 \cdot 3$ \\
\hline $1: 28: 16$ & 962.3 & 40.2 & 22.9 & 14.3 & 38.8 & $7 \cdot 1$ & 334.0 & 2.5 & 299.3 \\
\hline $\begin{array}{l}1: 28: 30 \\
1: 28: 43\end{array}$ & $\begin{array}{l}962.6 \\
962.9\end{array}$ & $\begin{array}{l}37.0 \\
34.3\end{array}$ & $\begin{array}{l}22.9 \\
22.9\end{array}$ & $\begin{array}{l}14 \cdot 3 \\
14.3\end{array}$ & $\begin{array}{l}38.4 \\
38.3\end{array}$ & $\begin{array}{l}7.0 \\
7.0\end{array}$ & $\begin{array}{l}333.4 \\
327.5\end{array}$ & $\begin{array}{l}2.4 \\
2.3\end{array}$ & $\begin{array}{l}299.3 \\
299.3\end{array}$ \\
\hline $1: 28: 56$ & 963.3 & 31.1 & 22.9 & 14.3 & 38.1 & 7.0 & 331.1 & 2.3 & 299.2 \\
\hline $1: 29: 9$ & 963.6 & 27.8 & 22.9 & $14 \cdot 3$ & 38.2 & 7.0 & 335.3 & 2.6 & 299.2 \\
\hline $1: 29: 22$ & 964.0 & 25.0 & 22.9 & 14.3 & 38.3 & 7.0 & 333 & 2.5 & 299.2 \\
\hline $1: 29: 36$ & 964.3 & 22.2 & 22.9 & $14 \cdot 3$ & 38.6 & 7.0 & 311.3 & 2.3 & 299.1 \\
\hline $1: 29: 49$ & 964.5 & 19.8 & 22.8 & $14 \cdot 3$ & 39.1 & $7 \cdot 1$ & $\begin{array}{l}313.5 \\
315.8\end{array}$ & $\begin{array}{l}2.5 \\
2.6\end{array}$ & $\begin{array}{l}299.0 \\
298.0\end{array}$ \\
\hline $\begin{array}{l}1: 30: 2 \\
1: 30: 15\end{array}$ & $\begin{array}{l}964.8 \\
965.1\end{array}$ & $\begin{array}{l}17.1 \\
14.7\end{array}$ & $\begin{array}{l}22.8 \\
22.8\end{array}$ & $\begin{array}{l}14.3 \\
14.3\end{array}$ & $\begin{array}{l}39.2 \\
39.2\end{array}$ & $\begin{array}{l}7.1 \\
7.1\end{array}$ & & $\begin{array}{l}2.6 \\
3.0\end{array}$ & $\begin{array}{l}298.9 \\
299.0\end{array}$ \\
\hline $1: 30: 28$ & 965.5 & 11.3 & 22.7 & 14.3 & 39.2 & 7.0 & 299.7 & 2.3 & 298.8 \\
\hline $1: 30: 42$ & 965 & 7.7 & 22.7 & 14.3 & 39.8 & 7.1 & 307.7 & 2.6 & 298.8 \\
\hline $1: 30: 55$ & 966.2 & 5.1 & 22.4 & 14.4 & 41.4 & $7 \cdot 3$ & 282.5 & 2.3 & 298.5 \\
\hline $1: 31: 8$ & 966.4 & $3 \cdot 3$ & 21.5 & 14.4 & 45.7 & 7. & & 1.2 & 297.6 \\
\hline $1: 31: 21$ & 967.1 & $-3 \cdot 3$ & 21.1 & 14.1 & 46.1 & 7.5 & 280.4 & 1.6 & 297.1 \\
\hline $1: 31: 34$ & 966.4 & 3.0 & 21.3 & 14.1 & 45.4 & & 52.1 & 0.8 & 297.3 \\
\hline $1: 31: 48$ & 966.5 & 2.0 & 20.5 & 14.1 & 49.7 & 7.8 & 81.7 & 0.2 & 296.5 \\
\hline $1: 32: 1$ & 966.5 & 1.9 & 20.1 & 14.0 & 51.0 & 7.8 & & 0.1 & 296.1 \\
\hline $1: 32: 14$ & 966.6 & 1.5 & 19.8 & 13.9 & 52.3 & 7.8 & 81.4 & 0.2 & 295.8 \\
\hline $1: 32: 27$ & 966.6 & 1.1 & 19.6 & 13.8 & 52.7 & 7.8 & 81.2 & 0.1 & \\
\hline $1: 32: 40$ & 966.5 & 1.6 & 19.5 & 13.7 & 52.4 & 7.7 & 81.4 & 0.1 & 295.5 \\
\hline $1: 32: 54$ & 966.5 & 1.6 & 19.3 & 13.6 & 53.3 & 7.8 & 81.4 & 0.3 & \\
\hline $1: 33: 7$ & 966.6 & 1.5 & 19.4 & 13.7 & 53.2 & 7. & 81.4 & 0.4 & \\
\hline $1: 33: 21$ & 966.6 & 1.6 & 19.3 & 13.6 & 52.9 & 7.7 & 81.3 & 0.1 & 295.3 \\
\hline
\end{tabular}

June 19-20, 1980; 2135-0234 PST

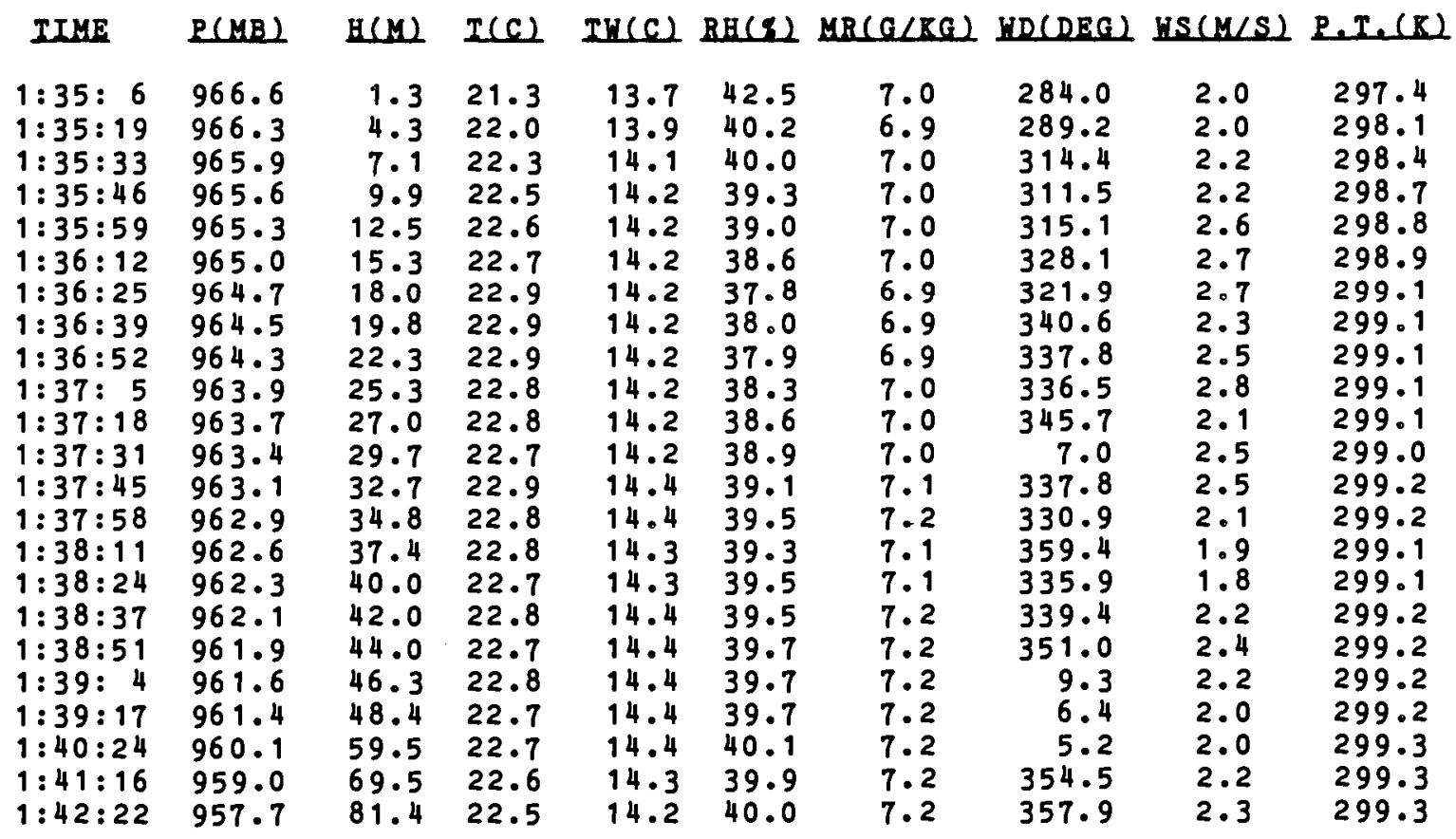




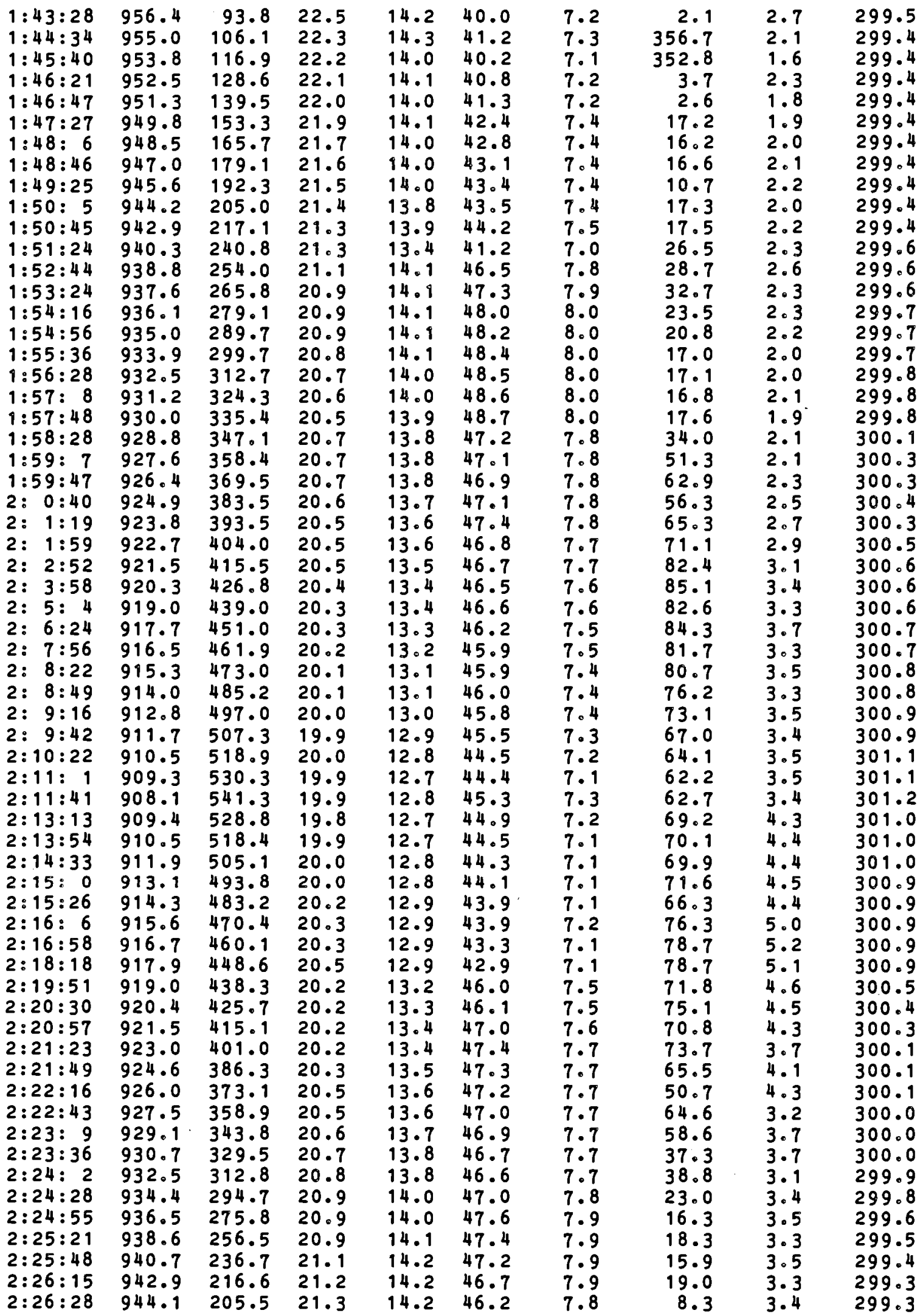




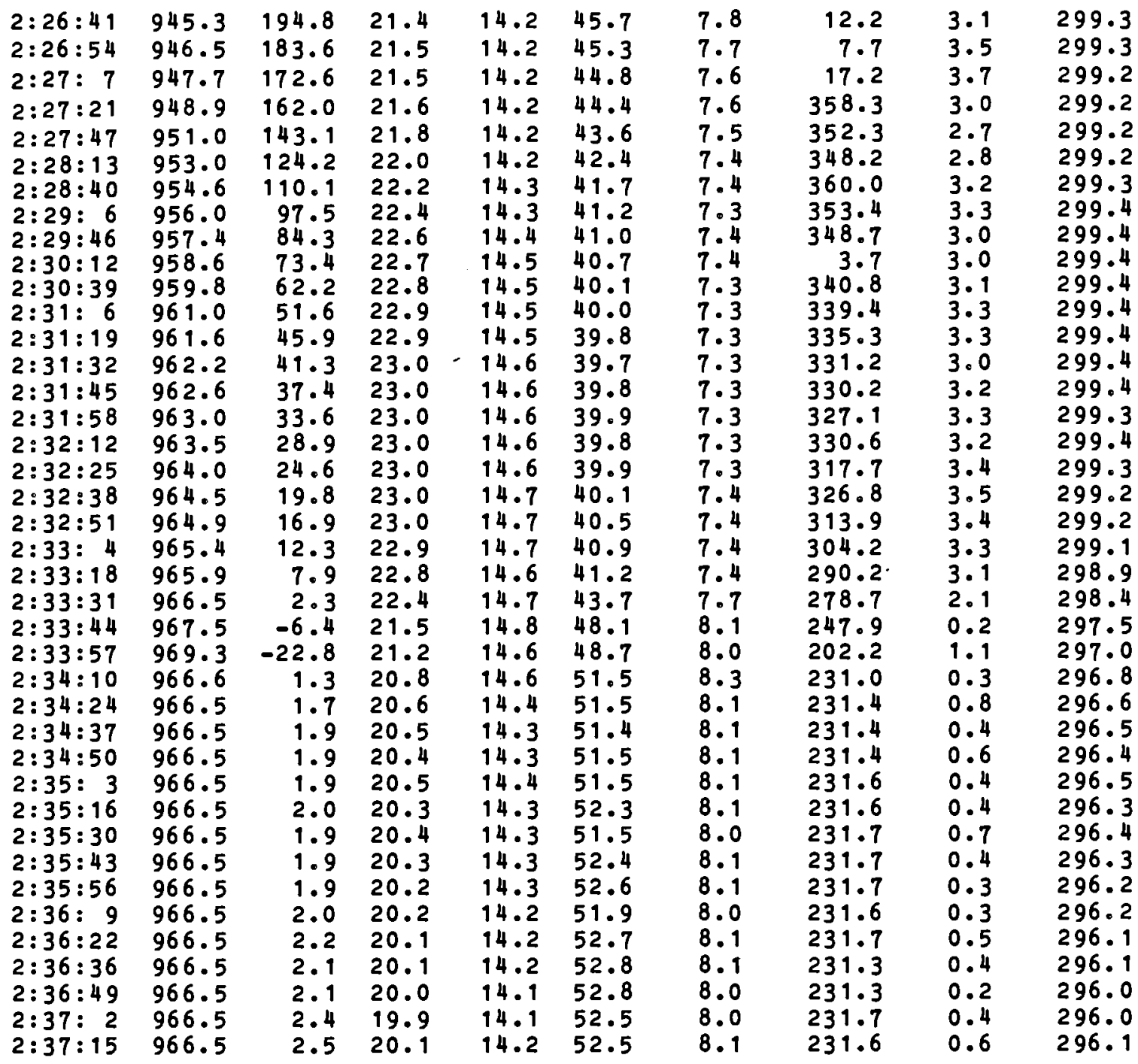


July 1-2, 1980: 1944-2027 RSI

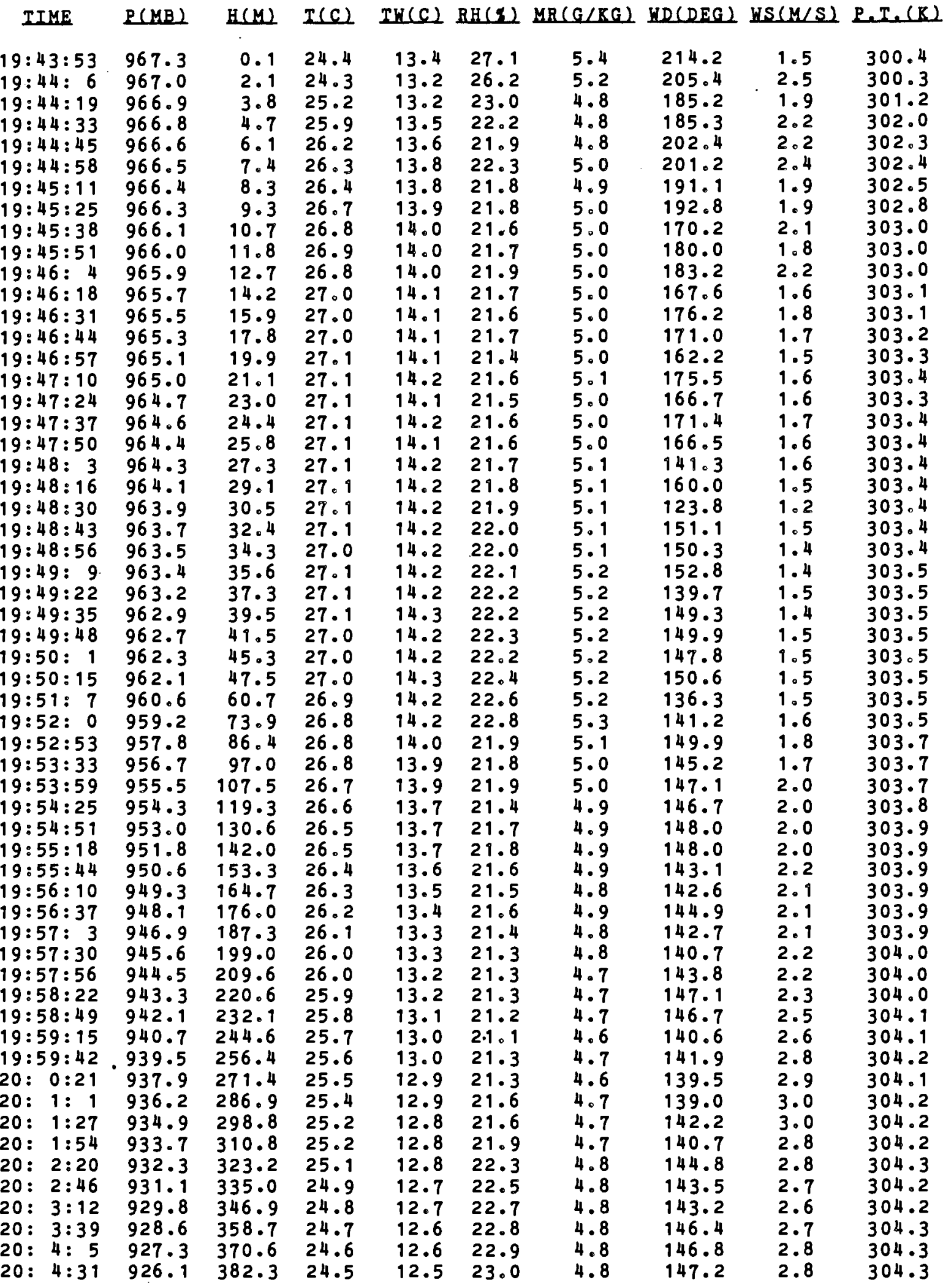









\begin{tabular}{|c|c|c|c|c|c|c|c|c|c|}
\hline $\begin{array}{l}20: 29: \quad 8 \\
20: 29: 21 \\
20: 29: 34 \\
20: 29: 48 \\
20: 30: \quad 1 \\
20: 30: 14 \\
20: 30: 27 \\
20: 30: 40 \\
20: 30: 54\end{array}$ & $\begin{array}{l}967.7 \\
967.8 \\
967.9 \\
967.9 \\
967.5 \\
967.6 \\
967.5 \\
967.5 \\
967.5\end{array}$ & $\begin{array}{l}-4 \cdot 1 \\
-4 \cdot 5 \\
-5 \cdot 8 \\
-5 \cdot 7 \\
-2 \cdot 2 \\
-2 \cdot 6 \\
-2 \cdot 1 \\
-2 \cdot 3 \\
-1.7\end{array}$ & $\begin{array}{l}24.2 \\
24.1 \\
24.2 \\
24.3 \\
24.0 \\
23.5 \\
23.3 \\
23.3 \\
23.2\end{array}$ & $\begin{array}{l}13.3 \\
13.3 \\
13.3 \\
13.4 \\
13.4 \\
13.2 \\
13.0 \\
13.0 \\
13.0\end{array}$ & $\begin{array}{l}27 \cdot 1 \\
27 \cdot 3 \\
27.5 \\
27 \cdot 5 \\
28.5 \\
29.4 \\
29.4 \\
29.0 \\
29.5\end{array}$ & $\begin{array}{l}5.3 \\
5.3 \\
5.4 \\
5.4 \\
5.5 \\
5.5 \\
5.4 \\
5.4 \\
5.5\end{array}$ & $\begin{array}{r}33.9 \\
31.5 \\
351.2 \\
29.7 \\
32.7 \\
32.1 \\
32.2 \\
32.3 \\
32.2\end{array}$ & $\begin{array}{l}1.7 \\
1.7 \\
1.7 \\
1.5 \\
0.8 \\
0.3 \\
0.7 \\
0.8 \\
0.5\end{array}$ & $\begin{array}{l}300.2 \\
300.1 \\
300.1 \\
300.2 \\
299.9 \\
299.4 \\
299.2 \\
299.3 \\
299.2\end{array}$ \\
\hline
\end{tabular}

Iuly 1-2, 1980: 2043-2114 PST

IIHE $P(M B) H(K) T(C) I H(C) R H(C) H R(G / K G) H D(D E G) H S(M / S) P E T(R)$

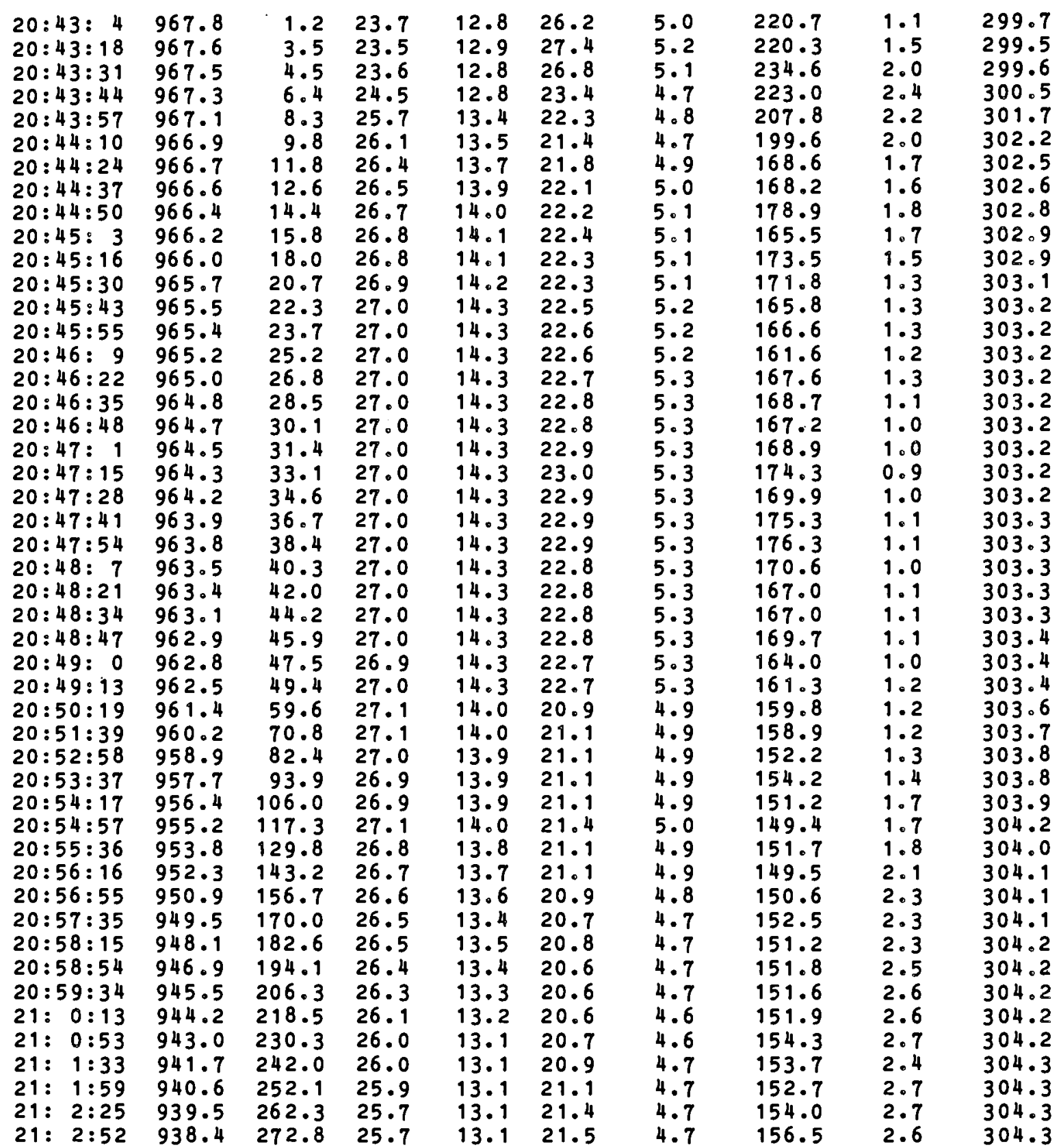




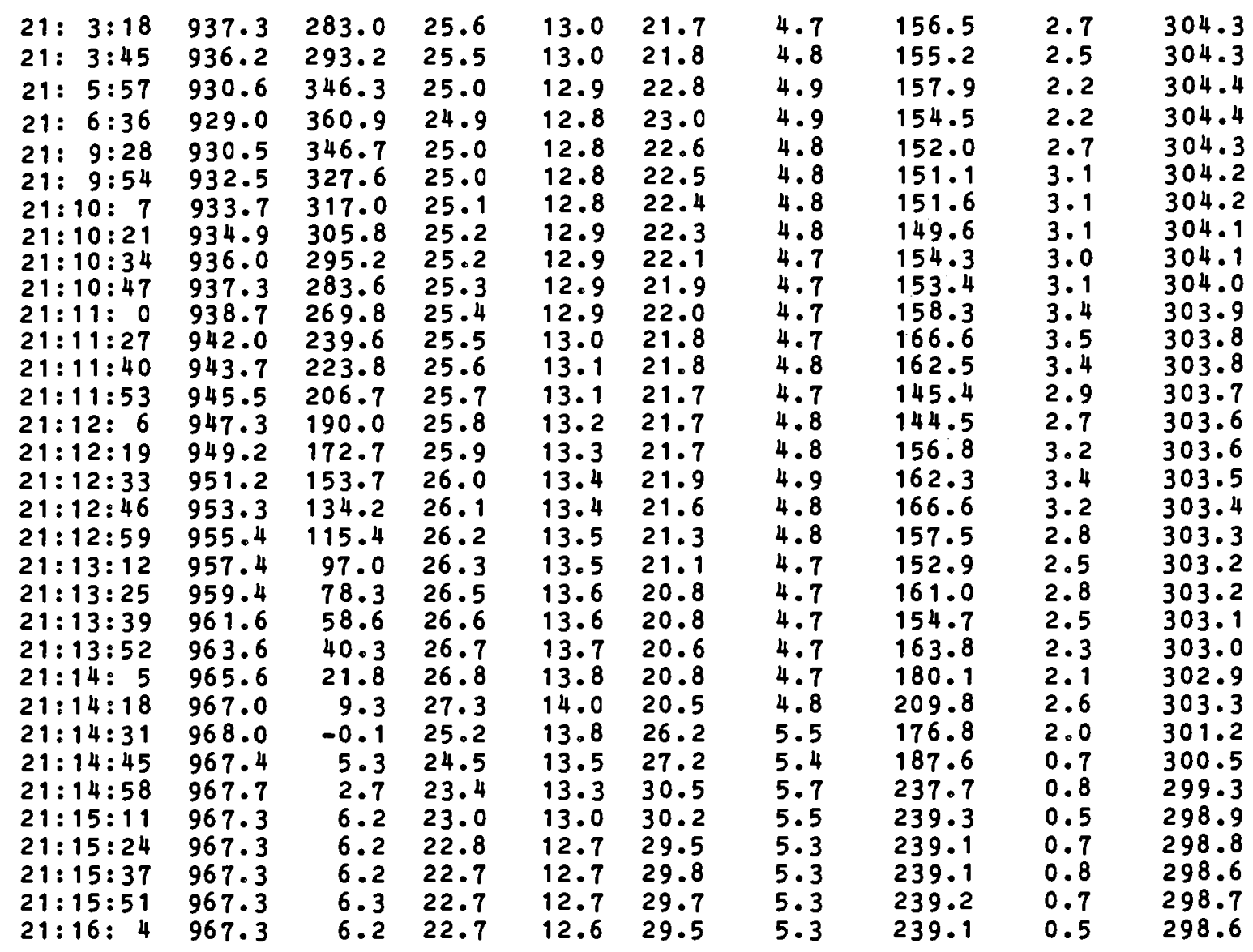

Iuly 1-2, 1980: 2124-2208 PST

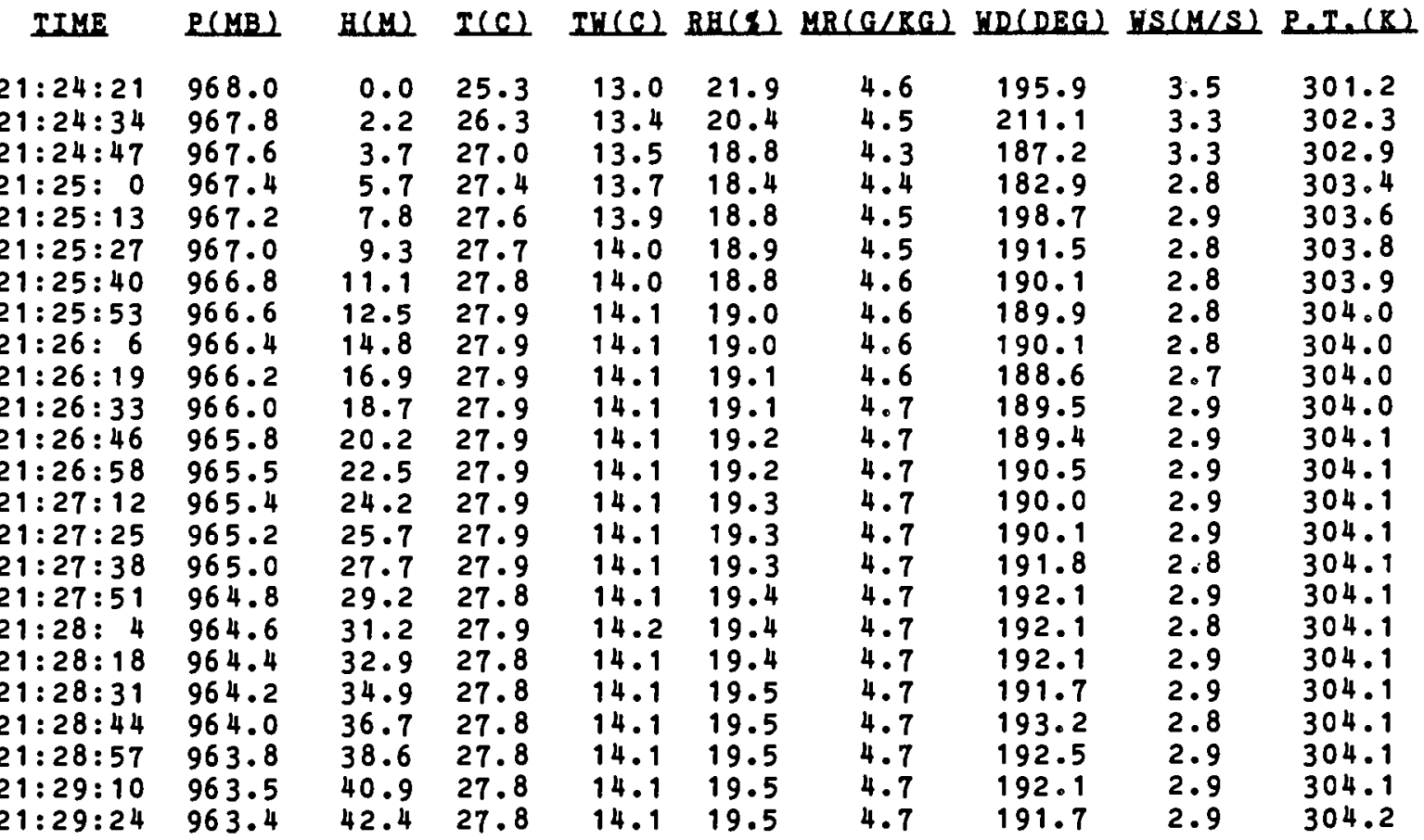




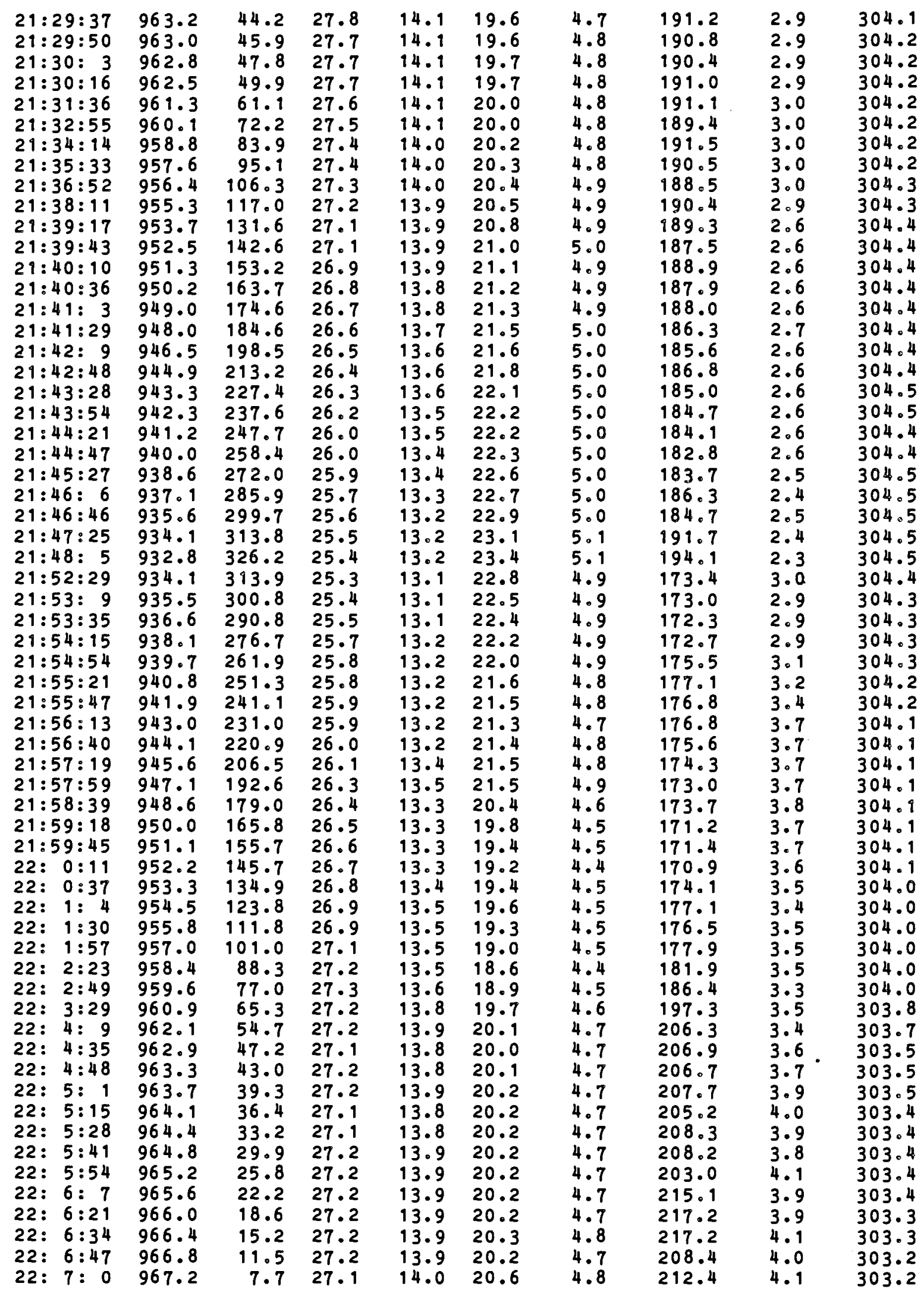




$\begin{array}{lllllllllll}22: 7: 13 & 967.6 & 4.2 & 26.5 & 14.1 & 23.4 & 5.2 & 239.6 & 3.4 & 302.5 \\ 22: 7: 27 & 967.9 & 1.4 & 24.4 & 13.6 & 28.3 & 5.6 & 234.4 & 2.1 & 300.3 \\ 22: 7: 40 & 968.6 & -5.0 & 23.6 & 13.2 & 28.7 & 5.4 & 237.0 & 2.0 & 299.5 \\ 22: 7: 53 & 967.7 & 3.6 & 23.4 & 13.1 & 29.6 & 5.5 & 241.2 & 0.8 & 299.3 \\ 22: 8: 6 & 967.6 & 4.0 & 23.0 & 12.9 & 29.8 & 5.4 & 241.2 & 0.9 & 298.9 \\ 22: 8: 19 & 967.6 & 4.2 & 23.0 & 12.9 & 29.5 & 5.4 & 241.4 & 0.6 & 299.0 \\ 22: 8: 33 & 967.6 & 3.9 & 22.8 & 12.8 & 29.9 & 5.4 & 241.2 & 0.5 & 298.8 \\ 22: 8: 46 & 967.6 & 3.8 & 22.8 & 12.8 & 30.0 & 5.4 & 241.1 & 0.5 & 298.8 \\ 22: 8: 59 & 967.6 & 4.0 & 22.6 & 12.7 & 30.2 & 5.4 & 241.1 & 0.6 & 298.6\end{array}$

July $1-2$. 1980; 2219-2304 RST

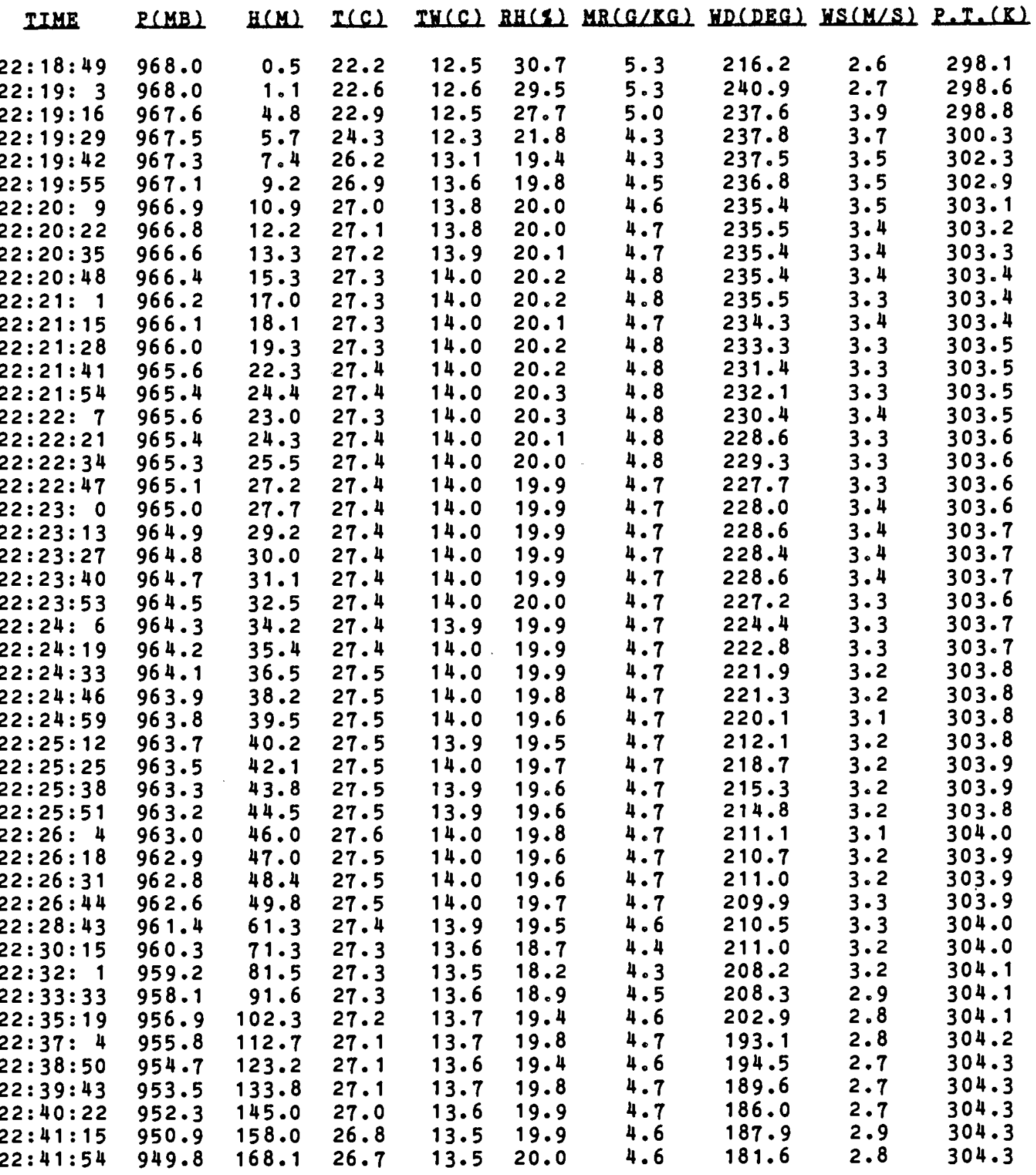




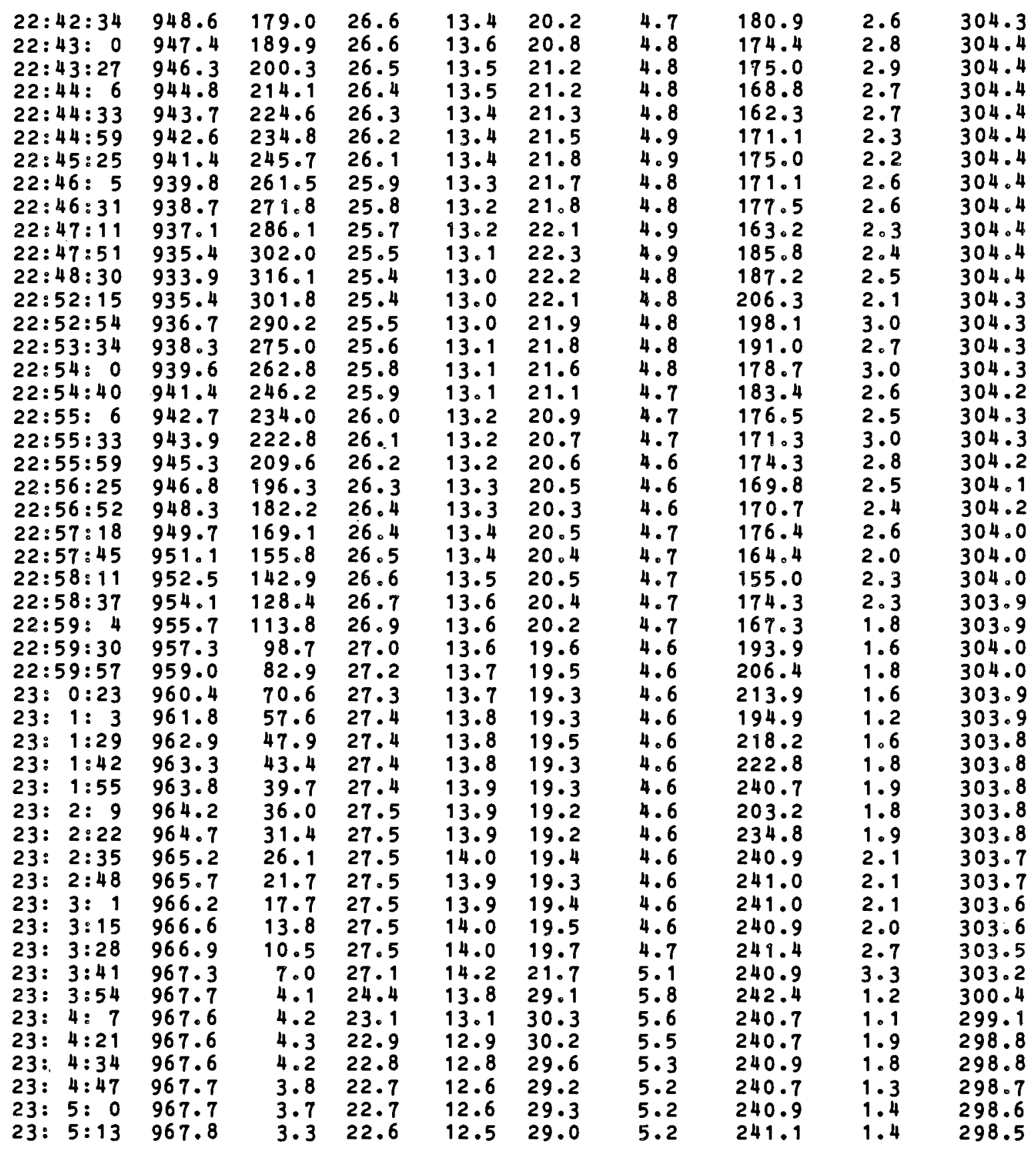

July 1-2. 1980: 2310-2348 PST

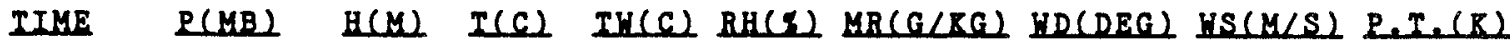

$\begin{array}{lrrrrrrrrr}23: 9: 54 & 968.2 & 0.8 & 22.0 & 12.4 & 30.8 & 5.3 & 240.8 & 2.6 & 297.9 \\ 23: 10: 7 & 967.7 & 5.1 & 23.8 & 11.9 & 20.7 & 4.0 & 249.6 & 2.9 & 299.8 \\ 23: 10: 21 & 967.4 & 7.3 & 25.5 & 12.8 & 20.1 & 4.3 & 239.3 & 2.7 & 301.5 \\ 23: 10: 34 & 967.4 & 7.7 & 26.5 & 13.3 & 19.4 & 4.3 & 196.5 & 1.5 & 302.5 \\ 23: 10: 47 & 967.0 & 11.5 & 26.7 & 13.5 & 19.7 & 4.5 & 126.9 & 0.8 & 302.7 \\ 23: 11: 0 & 966.7 & 13.9 & 26.8 & 13.7 & 20.2 & 4.6 & 240.1 & 0.5 & 302.9 \\ 23: 11: 13 & 966.5 & 16.3 & 26.9 & 13.7 & 20.4 & 4.7 & 165.7 & 1.3 & 303.0 \\ 23: 11: 27 & 966.0 & 20.0 & 26.8 & 13.8 & 21.1 & 4.8 & 166.0 & 1.1 & 302.9\end{array}$




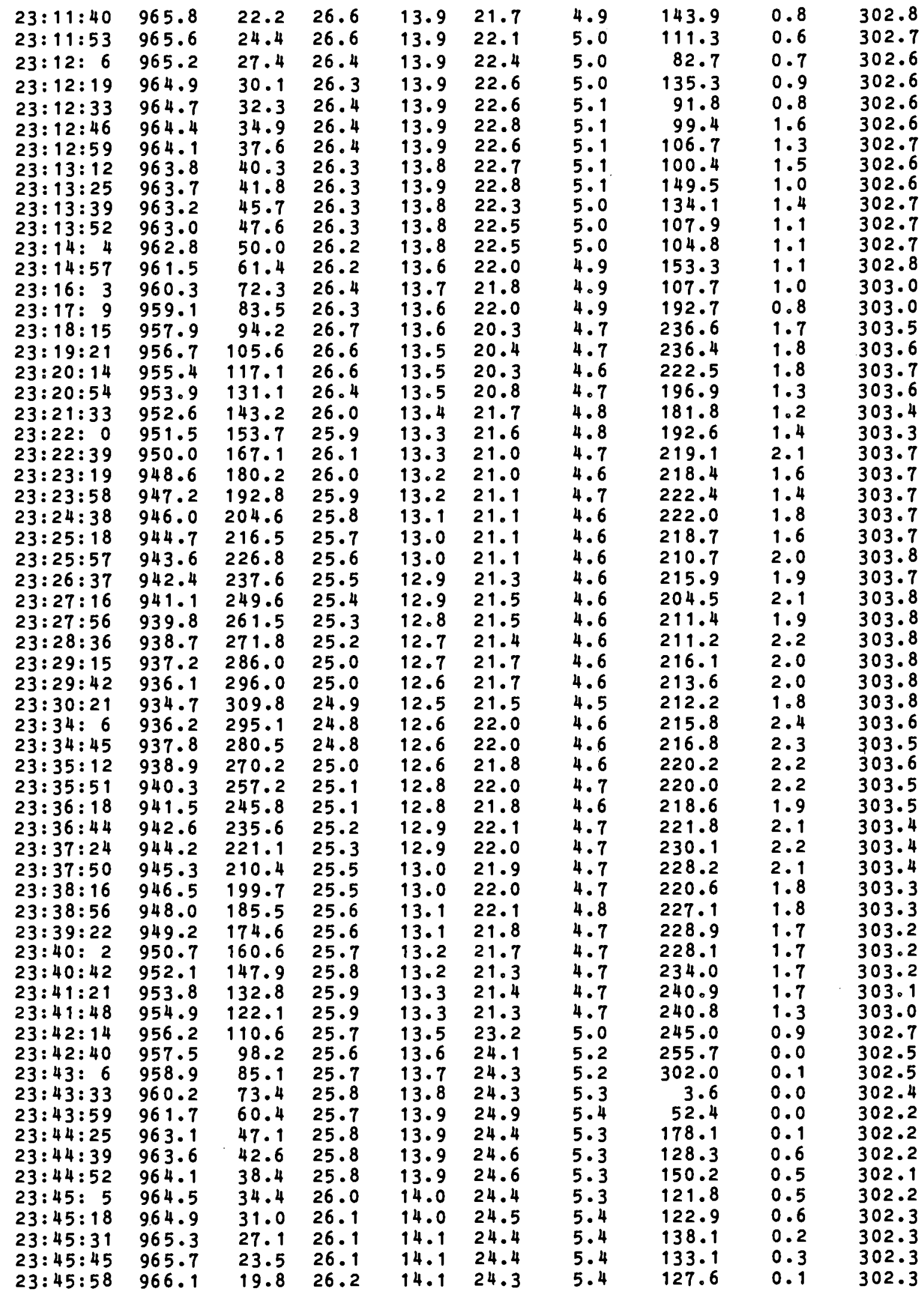




$\begin{array}{llllllllll}23: 46: 11 & 966.4 & 17.5 & 26.2 & 14.2 & 24.6 & 5.5 & 172.4 & 0.0 & 302.3 \\ 23: 46: 24 & 966.5 & 16.0 & 26.2 & 14.2 & 24.6 & 5.4 & 161.3 & 0.0 & 302.3 \\ 23: 46: 37 & 966.7 & 14.1 & 26.2 & 14.2 & 24.6 & 5.4 & 163.9 & 0.0 & 302.3 \\ 23: 46: 51 & 967.0 & 11.9 & 26.2 & 14.2 & 24.6 & 5.4 & 195.5 & 0.0 & 302.2 \\ 23: 47: 4 & 967.2 & 9.7 & 26.2 & 14.1 & 24.2 & 5.4 & 204.2 & 0.0 & 302.3 \\ 23: 47: 17 & 967.3 & 8.5 & 26.1 & 14.0 & 24.2 & 5.3 & 218.8 & 0.9 & 302.1 \\ 23: 47: 30 & 967.7 & 5.6 & 26.2 & 14.0 & 23.6 & 5.2 & 242.3 & 1.6 & 302.2 \\ 23: 47: 43 & 967.9 & 3.3 & 26.0 & 13.8 & 23.5 & 5.1 & 241.4 & 1.6 & 302.0 \\ 23: 47: 57 & 967.8 & 4.1 & 25.9 & 13.9 & 24.2 & 5.2 & 240.9 & 1.7 & 301.9 \\ 23: 48: 10 & 968.2 & 1.1 & 25.3 & 14.4 & 29.2 & 6.1 & 240.8 & 1.1 & 301.2 \\ 23: 48: 23 & 968.2 & 0.8 & 22.4 & 13.2 & 34.0 & 6.0 & 242.0 & 1.2 & 298.3 \\ 23: 48: 36 & 968.2 & 0.8 & 21.8 & 12.8 & 34.5 & 5.8 & 241.3 & 0.7 & 297.6 \\ 23: 49: 3 & 968.2 & 0.5 & 21.5 & 12.6 & 34.4 & 5.7 & 240.9 & 1.1 & 297.3 \\ 23: 49: 16 & 968.2 & 0.5 & 21.5 & 12.5 & 34.1 & 5.6 & 241.0 & 1.3 & 297.3\end{array}$

July 1-2, 1980: 2358-0042 RST

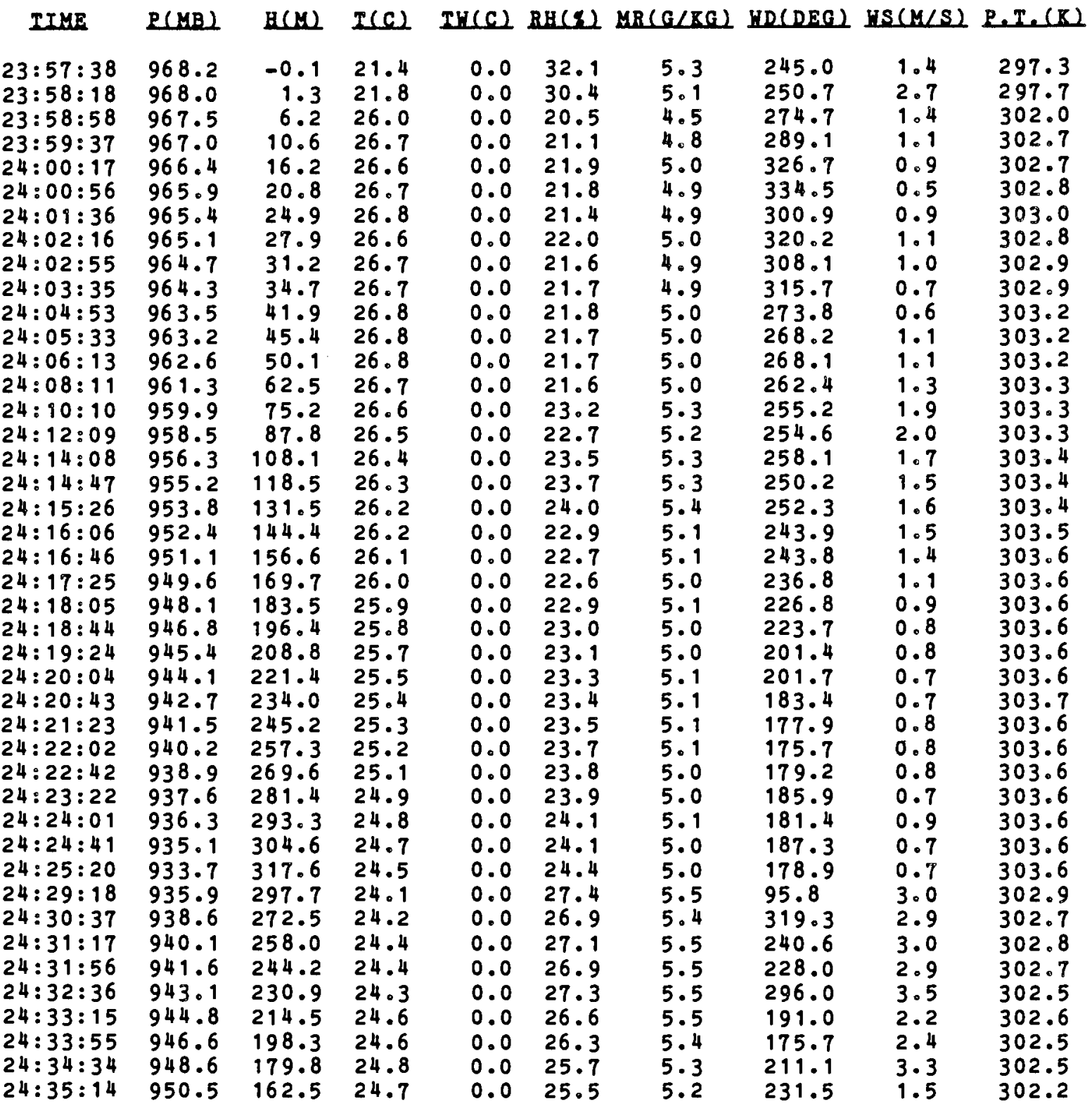




$\begin{array}{rrrrrrrrrr}24: 36: 33 & 954.3 & 126.9 & 25.1 & 0.0 & 24.6 & 5.1 & 54.9 & 1.5 & 302.2 \\ 24: 37: 13 & 956.8 & 103.9 & 25.2 & 0.0 & 25.0 & 5.2 & 209.7 & 1.0 & 302.1 \\ 24: 37: 52 & 958.7 & 87.3 & 25.0 & 0.0 & 24.9 & 5.2 & 185.9 & 2.5 & 301.8 \\ 24: 38: 32 & 960.4 & 71.7 & 25.3 & 0.0 & 23.6 & 5.0 & 158.2 & 1.8 & 302.0 \\ 24: 39: 11 & 961.7 & 59.2 & 25.5 & 0.0 & 23.2 & 5.0 & 126.6 & 1.1 & 302.0 \\ 24: 39: 51 & 963.2 & 46.1 & 25.3 & 0.0 & 24.1 & 5.0 & 156.1 & 1.7 & 301.6 \\ 24: 40: 31 & 965.1 & 28.7 & 25.3 & 0.0 & 24.1 & 5.0 & 57.4 & 0.2 & 301.4 \\ 24: 41: 10 & 966.7 & 14.1 & 25.2 & 0.0 & 25.1 & 5.2 & 250.9 & 1.0 & 301.3 \\ 24: 41: 50 & 967.6 & 6.0 & 24.7 & 0.0 & 27.3 & 5.5 & 244.7 & 2.8 & 300.7 \\ 24: 42: 29 & 968.1 & 1.1 & 22.5 & 0.0 & 32.7 & 5.8 & 243.4 & 1.5 & 298.4 \\ 24: 43: 09 & 968.1 & 1.8 & 22.1 & 0.0 & 32.7 & 5.6 & 242.0 & 1.0 & 298.0 \\ 24: 43: 49 & 968.1 & 1.2 & 21.4 & 0.0 & 35.2 & 5.8 & 257.5 & 1.0 & 297.3\end{array}$

July 1-2, 1980; 0050-0133 RST

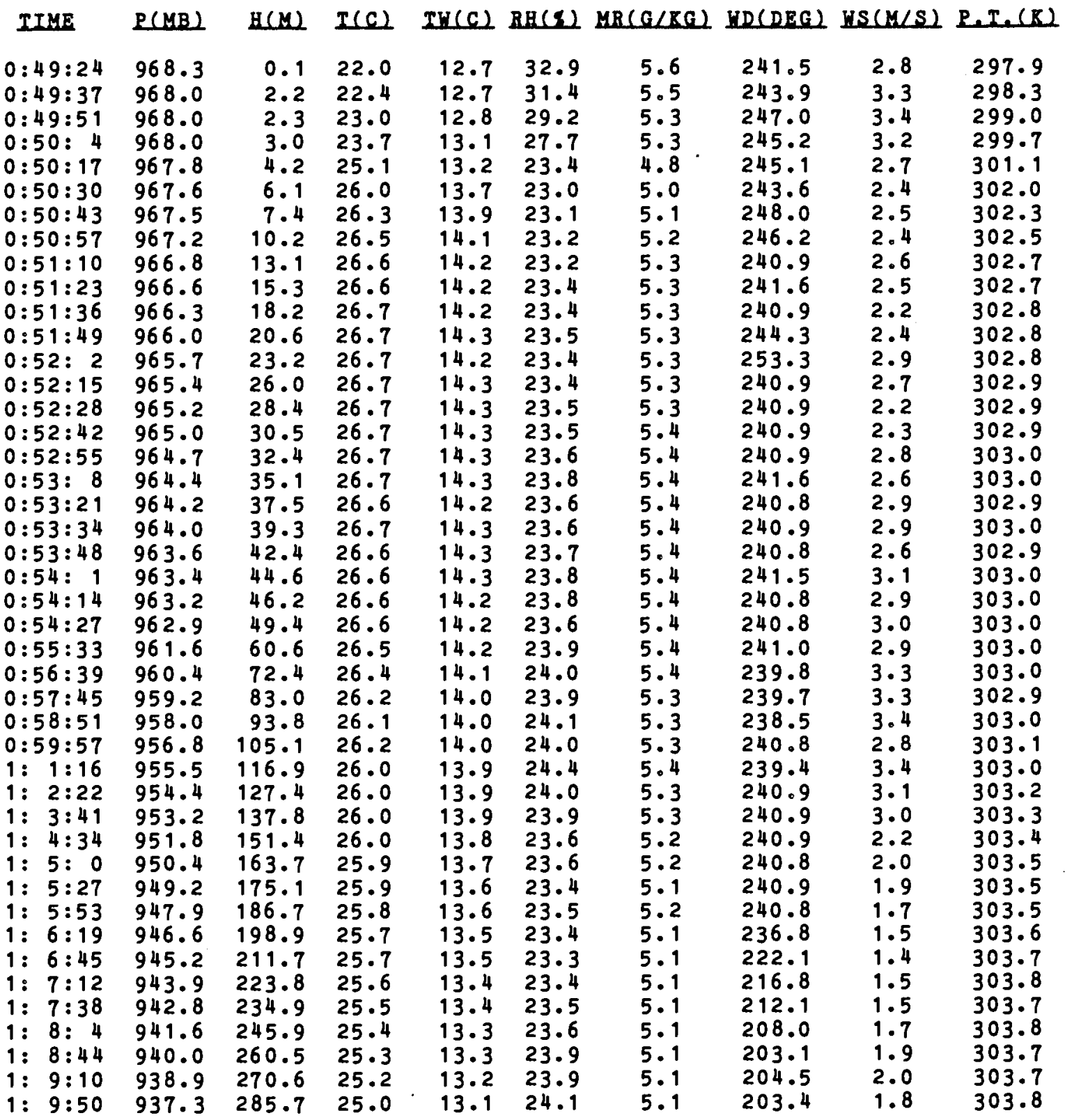




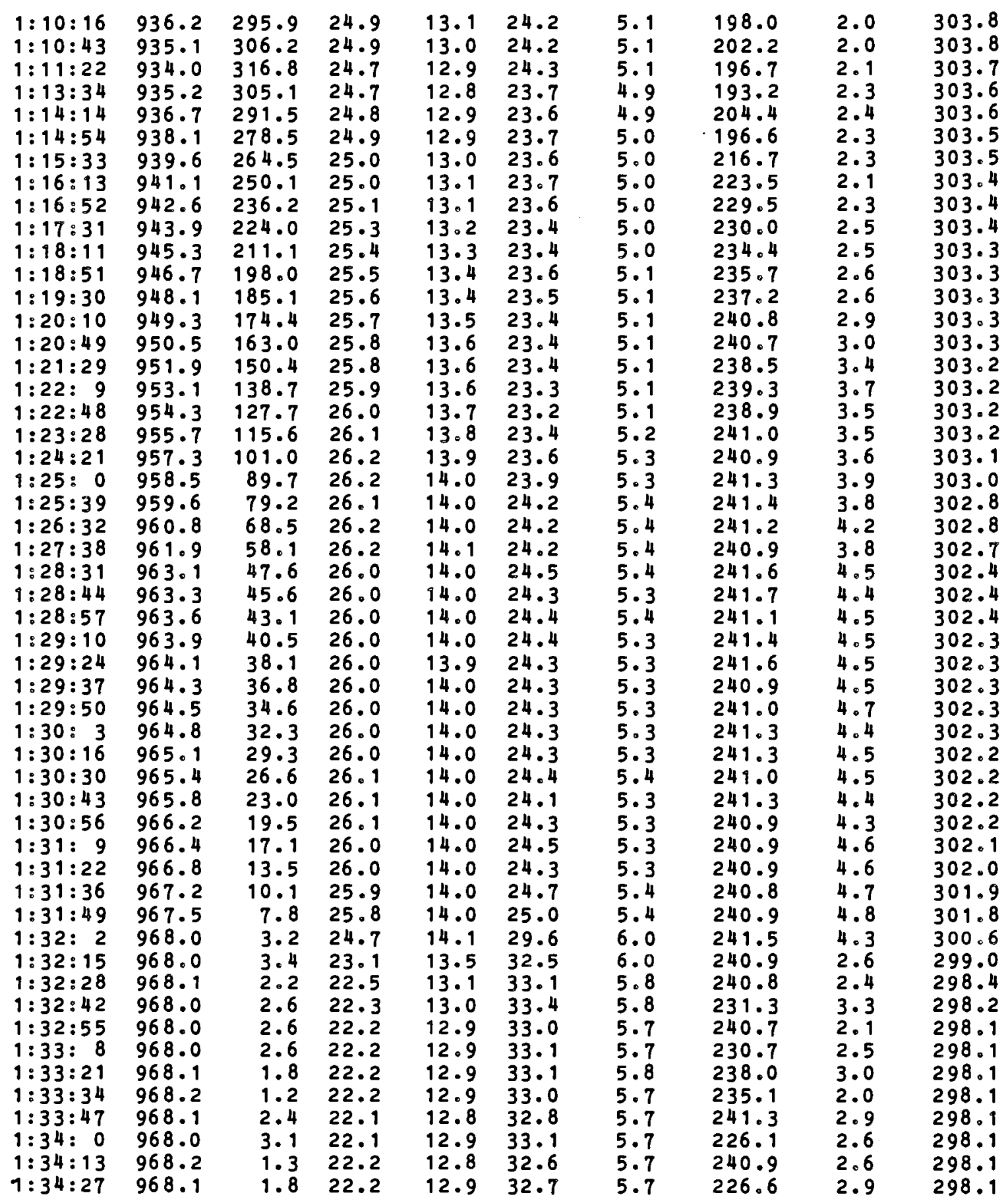


July 2-3, 1981: 1949-2026 PSI

IIME $P(M B) \quad H(M) \quad I(C) \quad I W(C) R H(G) M R(G / B G)$ WD(DEG) $W S(M / S) R . T .(B)$

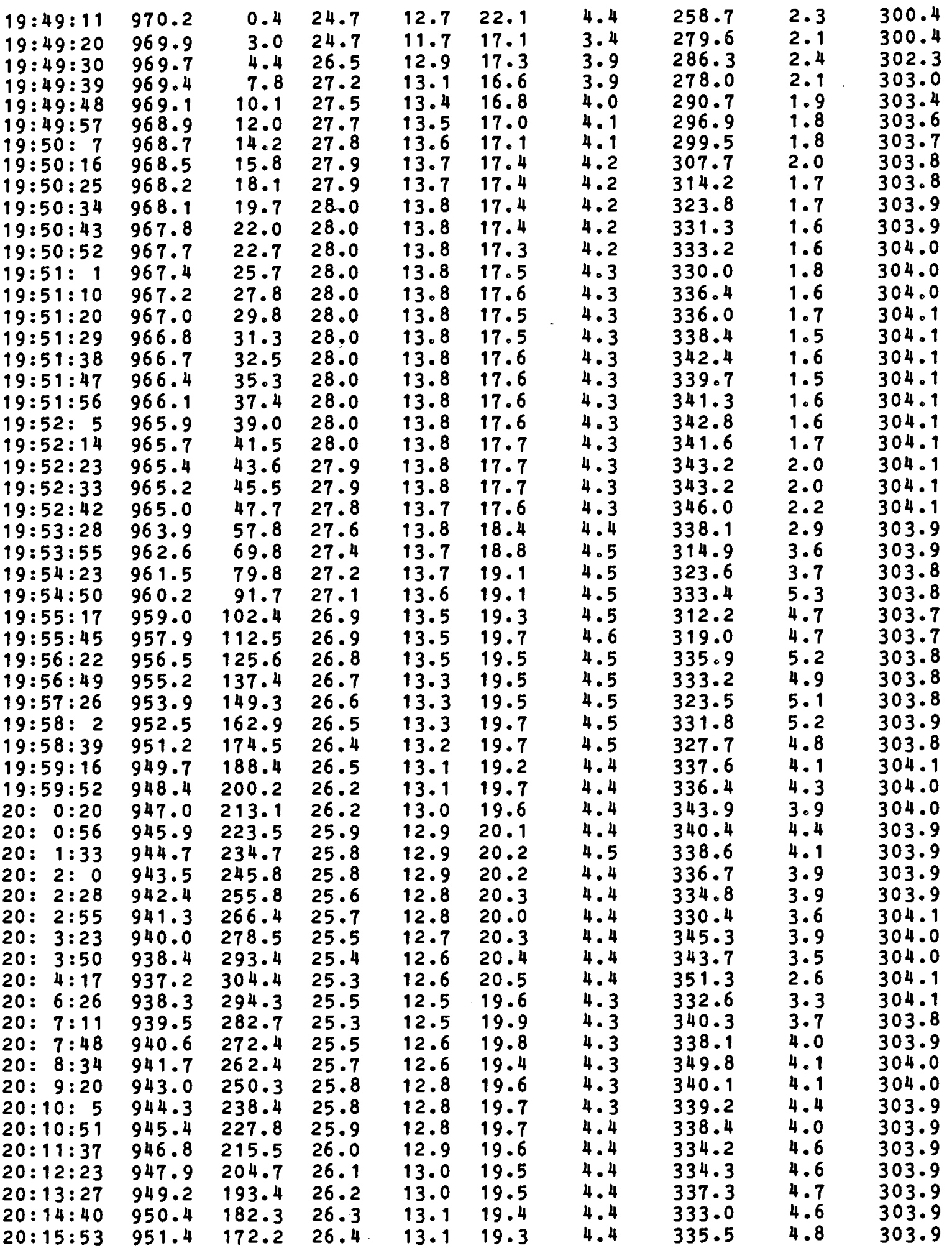




\begin{tabular}{|c|c|c|c|c|c|c|c|c|c|}
\hline $\begin{array}{l}20: 16: 30 \\
20: 17: 7 \\
20: 17: 43 \\
20: 18: 20 \\
20: 18: 47 \\
20: 19: 24 \\
20: 20: 10 \\
20: 20: 55 \\
20: 21: 32 \\
20: 22: 8 \\
20: 22: 36 \\
20: 22: 45 \\
20: 22: 55 \\
20: 23: 4 \\
20: 23: 13 \\
20: 23: 22 \\
20: 23: 31 \\
20: 23: 40 \\
20: 23: 49 \\
20: 23: 52 \\
20: 24: 8 \\
20: 24: 17 \\
20: 24: 26 \\
20: 24: 35 \\
20: 24: 44 \\
20: 24: 53 \\
20: 25: 22 \\
20: 25: 11 \\
20: 25: 21 \\
20: 25: 30 \\
20: 25: 39 \\
20: 25: 48 \\
20: 25: 58 \\
20: 26: 7 \\
20: 26: 16 \\
20: 26: 25 \\
20: 26: 34 \\
20: 26: 43 \\
20: 26: 52\end{array}$ & $\begin{array}{l}952.8 \\
954.2 \\
955.6 \\
957.1 \\
958.2 \\
959.4 \\
960.5 \\
961.8 \\
962.9 \\
964.0 \\
964.8 \\
965.0 \\
965.3 \\
965.5 \\
965.9 \\
966.1 \\
966.2 \\
966.4 \\
966.7 \\
966.9 \\
967.1 \\
967.4 \\
967.6 \\
967.9 \\
968.3 \\
968.5 \\
969.0 \\
969.3 \\
969.3 \\
969.5 \\
969.8 \\
970.1 \\
969.4 \\
969.9 \\
969.8 \\
969.8 \\
969.8 \\
969.8 \\
969.8\end{array}$ & $\begin{array}{r}159.7 \\
146.5 \\
133.7 \\
120.1 \\
110.0 \\
99.2 \\
88.6 \\
77.1 \\
67.1 \\
56.6 \\
49.3 \\
47.2 \\
45.1 \\
43.0 \\
39.8 \\
37.4 \\
37.1 \\
34.7 \\
32.3 \\
30.5 \\
28.2 \\
26.1 \\
24.0 \\
21.5 \\
17.7 \\
15.7 \\
10.9 \\
8.5 \\
8.1 \\
6.4 \\
4.1 \\
0.8 \\
7.4 \\
3.1 \\
3.9 \\
3.8 \\
3.6 \\
3.6 \\
3.6\end{array}$ & $\begin{array}{l}26 \cdot 5 \\
26 \cdot 7 \\
26.8 \\
26.9 \\
26.9 \\
27 \cdot 0 \\
27 \cdot 1 \\
27 \cdot 3 \\
27.4 \\
27.4 \\
27 \cdot 5 \\
27 \cdot 5 \\
27.5 \\
27.5 \\
27.4 \\
27.6 \\
27.6 \\
27.6 \\
27.5 \\
27.6 \\
27.5 \\
27.4 \\
27 \cdot 5 \\
27.6 \\
27.5 \\
27.6 \\
27.5 \\
27.2 \\
27.2 \\
27.1 \\
26.8 \\
26.7 \\
26.0 \\
26.0 \\
25.2 \\
25.0 \\
25.0 \\
25.0 \\
24.9\end{array}$ & $\begin{array}{l}13.2 \\
13.3 \\
13.3 \\
13.4 \\
13.4 \\
13.5 \\
13.6 \\
13.8 \\
13.9 \\
13.9 \\
14.0 \\
14.0 \\
14.0 \\
14.1 \\
14.0 \\
14.1 \\
14.1 \\
14.1 \\
14.1 \\
14.2 \\
14.1 \\
14.1 \\
14.1 \\
14.1 \\
14.1 \\
14.2 \\
14.2 \\
14.1 \\
14.1 \\
14.2 \\
14.1 \\
14.0 \\
14.0 \\
14.0 \\
13.8 \\
13.6 \\
13.6 \\
13.5 \\
13.5\end{array}$ & $\begin{array}{l}19.2 \\
19.1 \\
19.1 \\
19.1 \\
19.0 \\
19.0 \\
19.2 \\
19.4 \\
19.6 \\
19.5 \\
19.7 \\
19.8 \\
19.8 \\
19.9 \\
20.0 \\
19.8 \\
19.9 \\
19.9 \\
20.2 \\
20.4 \\
20.1 \\
20.4 \\
20.2 \\
20.1 \\
20.2 \\
20.3 \\
20.6 \\
21.3 \\
21.0 \\
21.6 \\
22.0 \\
22.4 \\
24.1 \\
24.5 \\
26.3 \\
25.4 \\
25.6 \\
25.3 \\
25.6\end{array}$ & $\begin{array}{l}4.4 \\
4.4 \\
4.4 \\
4.4 \\
4.4 \\
4.4 \\
4.5 \\
4.6 \\
4.6 \\
4.6 \\
4.7 \\
4.7 \\
4.7 \\
4.7 \\
4.7 \\
4.8 \\
4.8 \\
4.8 \\
4.8 \\
4.9 \\
4.8 \\
4.8 \\
4.8 \\
4.8 \\
4.8 \\
4.8 \\
4.9 \\
5.0 \\
4.9 \\
5.0 \\
5.0 \\
5.1 \\
5.2 \\
5.3 \\
5.4 \\
5.2 \\
5.3 \\
5.2 \\
5.2\end{array}$ & $\begin{array}{l}335.5 \\
334.1 \\
335.5 \\
330.0 \\
329.9 \\
330.4 \\
327.2 \\
331.9 \\
330.6 \\
328.4 \\
327.8 \\
329.1 \\
327.9 \\
333.8 \\
329.2 \\
327.8 \\
317.7 \\
342.3 \\
316.1 \\
335.5 \\
343.9 \\
319.1 \\
312.2 \\
315.1 \\
318.3 \\
307.0 \\
311.6 \\
329.9 \\
320.8 \\
300.1 \\
277.4 \\
291.2 \\
308.0 \\
318.5 \\
318.6 \\
318.7 \\
318.6 \\
318.6 \\
318.7\end{array}$ & $\begin{array}{l}4.5 \\
4.5 \\
4.5 \\
4.5 \\
4.2 \\
4.4 \\
4.1 \\
4.3 \\
4.0 \\
4.0 \\
3.8 \\
4.1 \\
4.0 \\
3.6 \\
3.8 \\
3.9 \\
4.1 \\
3.6 \\
3.4 \\
3.7 \\
3.8 \\
3.6 \\
3.6 \\
3.7 \\
3.6 \\
3.7 \\
3.4 \\
2.9 \\
3.1 \\
3.0 \\
3.0 \\
2.2 \\
1.2 \\
0.7 \\
0.4 \\
0.5 \\
0.1 \\
0.3 \\
0.5\end{array}$ & $\begin{array}{l}303.8 \\
303.9 \\
303.8 \\
303.8 \\
303.8 \\
303.8 \\
303.8 \\
303.8 \\
303.8 \\
303.7 \\
303.8 \\
303.7 \\
303.7 \\
303.7 \\
303.6 \\
303.8 \\
303.7 \\
303.7 \\
303.6 \\
303.6 \\
303.5 \\
303.4 \\
303.4 \\
303.5 \\
303.5 \\
303.5 \\
303.4 \\
303.0 \\
303.0 \\
302.9 \\
302.6 \\
302.4 \\
301.9 \\
301.9 \\
301.0 \\
300.8 \\
300.8 \\
300.8 \\
300.7\end{array}$ \\
\hline
\end{tabular}

Iuly 2-3. 1981: 2034-2110 RST

\begin{tabular}{|c|c|c|c|c|c|c|c|c|c|}
\hline LIME & $P(M B)$ & $\mathrm{H}(\mathrm{M})$ & $I(C)$ & $\operatorname{IH}(c)$ & $\mathrm{BH}(x)$ & $M R(G / K G)$ & HDC DEG & $H S(M / S)$ & R.T. $(K)$ \\
\hline $\begin{array}{l}0: 34: 13 \\
0: 34: 22 \\
0: 34: 31 \\
0: 34: 40 \\
0: 34: 49 \\
0: 34: 58 \\
0: 35: 7 \\
:=35: 17 \\
0: 35: 26 \\
0: 35: 35 \\
0: 35: 44 \\
0: 35: 53 \\
0: 36: 2 \\
0: 36: 11 \\
0: 36: 20 \\
0: 36: 30 \\
0: 36: 39 \\
0: 36: 48\end{array}$ & $\begin{array}{l}970.4 \\
970.0 \\
969.8 \\
969.3 \\
969.1 \\
968.7 \\
968.3 \\
968.0 \\
967.6 \\
967.4 \\
966.9 \\
966.5 \\
966.2 \\
965.9 \\
965.7 \\
965.6 \\
965.4 \\
965.2\end{array}$ & $\begin{array}{l}0.0 \\
3.3 \\
5.7 \\
10.0 \\
11.9 \\
15.5 \\
19.5 \\
21.9 \\
25.7 \\
27.6 \\
32.1 \\
35.5 \\
38.5 \\
40.9 \\
42.6 \\
44.0 \\
45.5 \\
47.4\end{array}$ & $\begin{array}{l}25.9 \\
26.6 \\
27.1 \\
27.2 \\
27.3 \\
27.3 \\
27.4 \\
27.3 \\
27.4 \\
27.4 \\
27.4 \\
27.4 \\
27.5 \\
27.5 \\
27.5 \\
27.5 \\
27.5 \\
27.5\end{array}$ & $\begin{array}{l}13.0 \\
13.3 \\
13.5 \\
13.6 \\
13.7 \\
13.8 \\
13.8 \\
13.8 \\
13.8 \\
13.8 \\
13.8 \\
13.8 \\
13.9 \\
13.8 \\
13.9 \\
13.8 \\
13.8 \\
13.8\end{array}$ & $\begin{array}{l}19.8 \\
18.7 \\
18.7 \\
19.0 \\
18.9 \\
19.0 \\
19.2 \\
19.2 \\
19.2 \\
19.1 \\
19.3 \\
18.9 \\
19.0 \\
18.9 \\
19.0 \\
19.1 \\
19.0 \\
19.0\end{array}$ & $\begin{array}{l}4.3 \\
4.2 \\
4.3 \\
4.4 \\
4.4 \\
4.5 \\
4.5 \\
4.5 \\
4.5 \\
4.5 \\
4.6 \\
4.5 \\
4.5 \\
4.5 \\
4.5 \\
4.5 \\
4.5 \\
4.5\end{array}$ & $\begin{array}{l}305.4 \\
301.5 \\
306.5 \\
301.1 \\
326.0 \\
312.9 \\
314.6 \\
307.3 \\
320.0 \\
327.9 \\
329.2 \\
330.9 \\
338.1 \\
330.9 \\
328.4 \\
331.7 \\
330.3 \\
328.6\end{array}$ & $\begin{array}{l}3.2 \\
3.6 \\
3.3 \\
3.3 \\
3.1 \\
3.6 \\
3.3 \\
3.2 \\
3.4 \\
4.0 \\
3.5 \\
3.8 \\
3.9 \\
4.0 \\
3.8 \\
3.9 \\
4.0 \\
3.9\end{array}$ & $\begin{array}{l}301.7 \\
302.4 \\
302.9 \\
303.0 \\
303.2 \\
303.2 \\
303.3 \\
303.3 \\
303.4 \\
303.4 \\
303.4 \\
303.5 \\
303.6 \\
303.6 \\
303.7 \\
303.6 \\
303.7 \\
303.7\end{array}$ \\
\hline
\end{tabular}




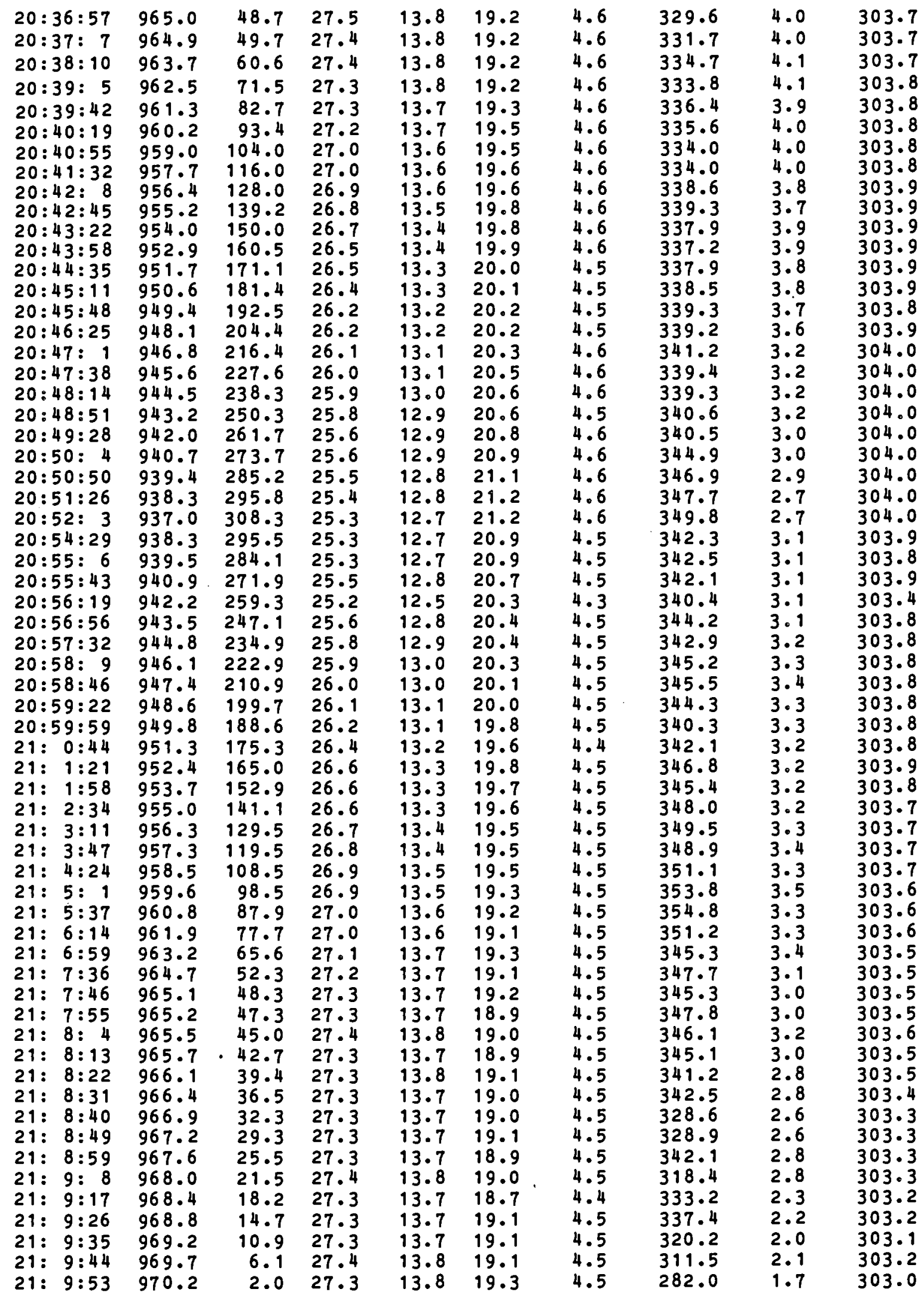




$\begin{array}{llllllllll}21: 10: 2 & 970.6 & -2.0 & 27.3 & 13.8 & 19.5 & 4.6 & 278.3 & 1.9 & 303.0 \\ 21: 10: 12 & 971.1 & -6.2 & 26.9 & 13.9 & 21.2 & 4.9 & 278.0 & 1.4 & 302.6 \\ 21: 10: 21 & 971.1 & -6.0 & 25.3 & 13.4 & 23.8 & 5.0 & 266.2 & 1.1 & 301.0 \\ 21: 10: 30 & 971.1 & -6.1 & 24.8 & 13.2 & 24.7 & 5.0 & 258.7 & 1.2 & 300.4 \\ 21: 10: 39 & 971.1 & -6.2 & 24.4 & 13.0 & 24.4 & 4.8 & 252.8 & 1.1 & 300.1 \\ 21: 10: 49 & 971.1 & -6.4 & 24.2 & 12.8 & 24.7 & 4.8 & 249.8 & 1.6 & 299.8 \\ 21: 10: 58 & 971.0 & -5.8 & 24.5 & 12.8 & 23.4 & 4.6 & 254.7 & 1.1 & 300.1 \\ 21: 11: 7 & 971.0 & -5.8 & 24.9 & 12.6 & 21.2 & 4.3 & 261.0 & 1.0 & 300.6 \\ 21: 11: 16 & 971.0 & -5.9 & 25.0 & 13.0 & 22.6 & 4.6 & 269.6 & 1.3 & 300.7\end{array}$

July 2-3, 1981: 2120-2151 PST

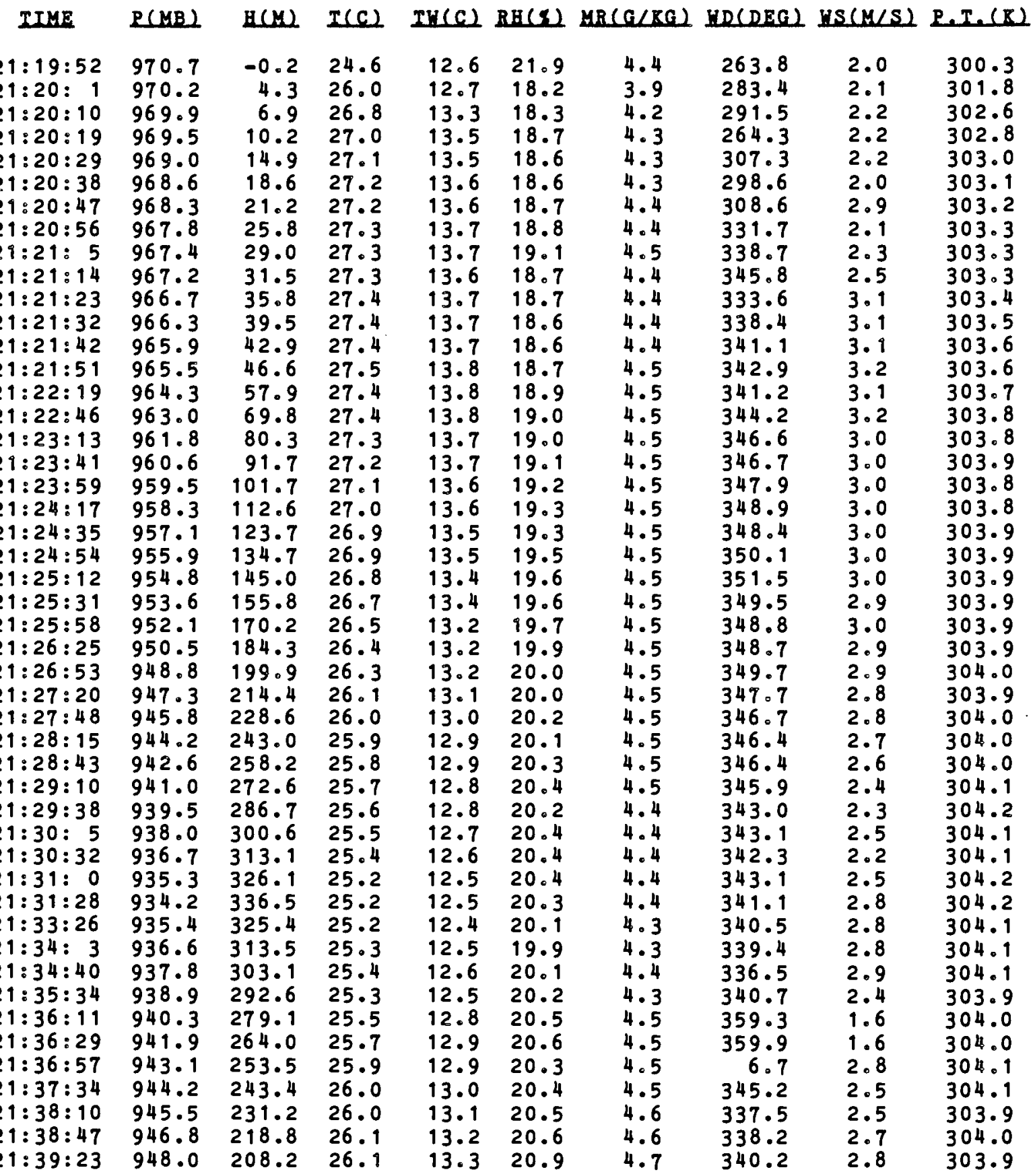




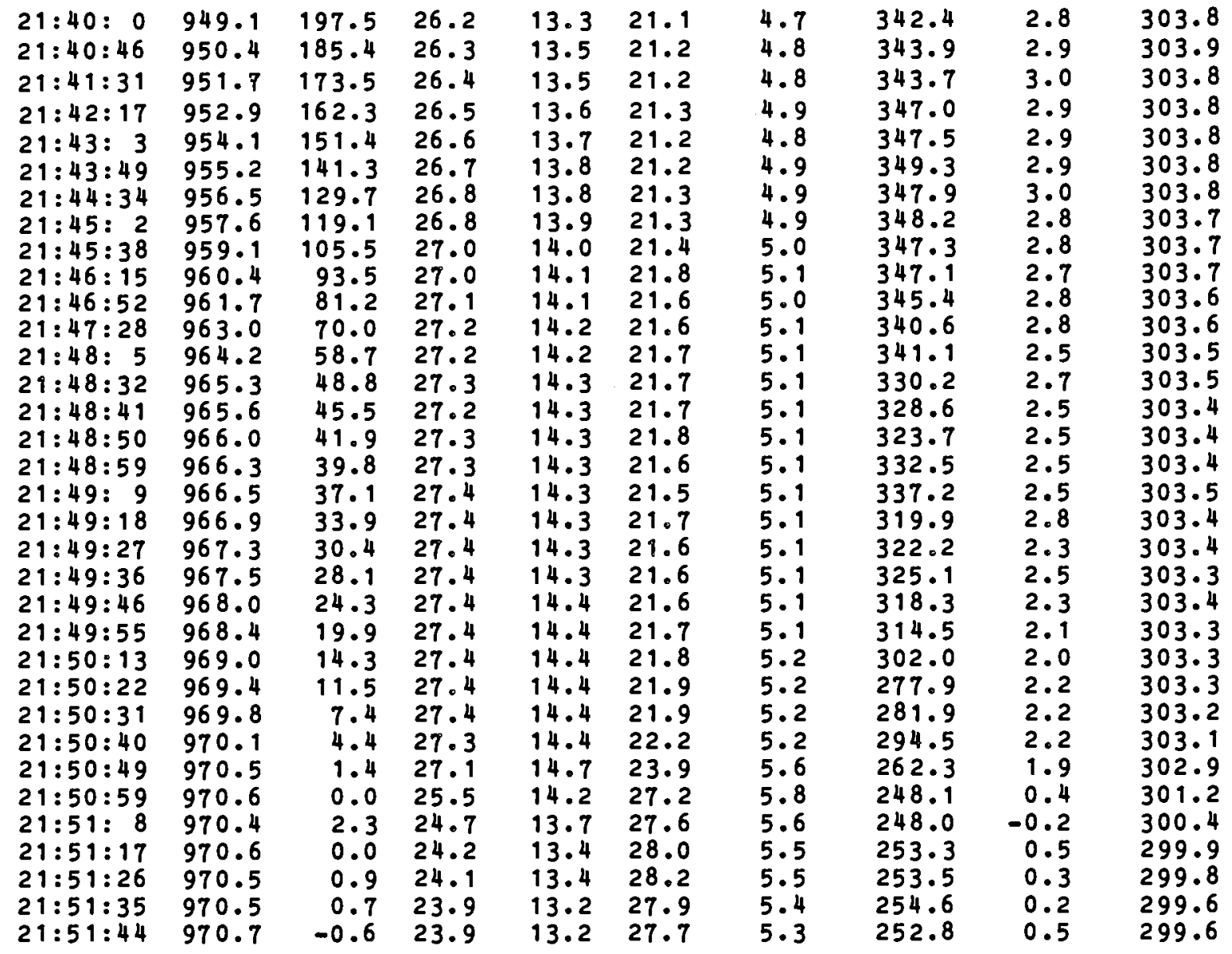

July 2-3. 1981: 2203-2240 PST

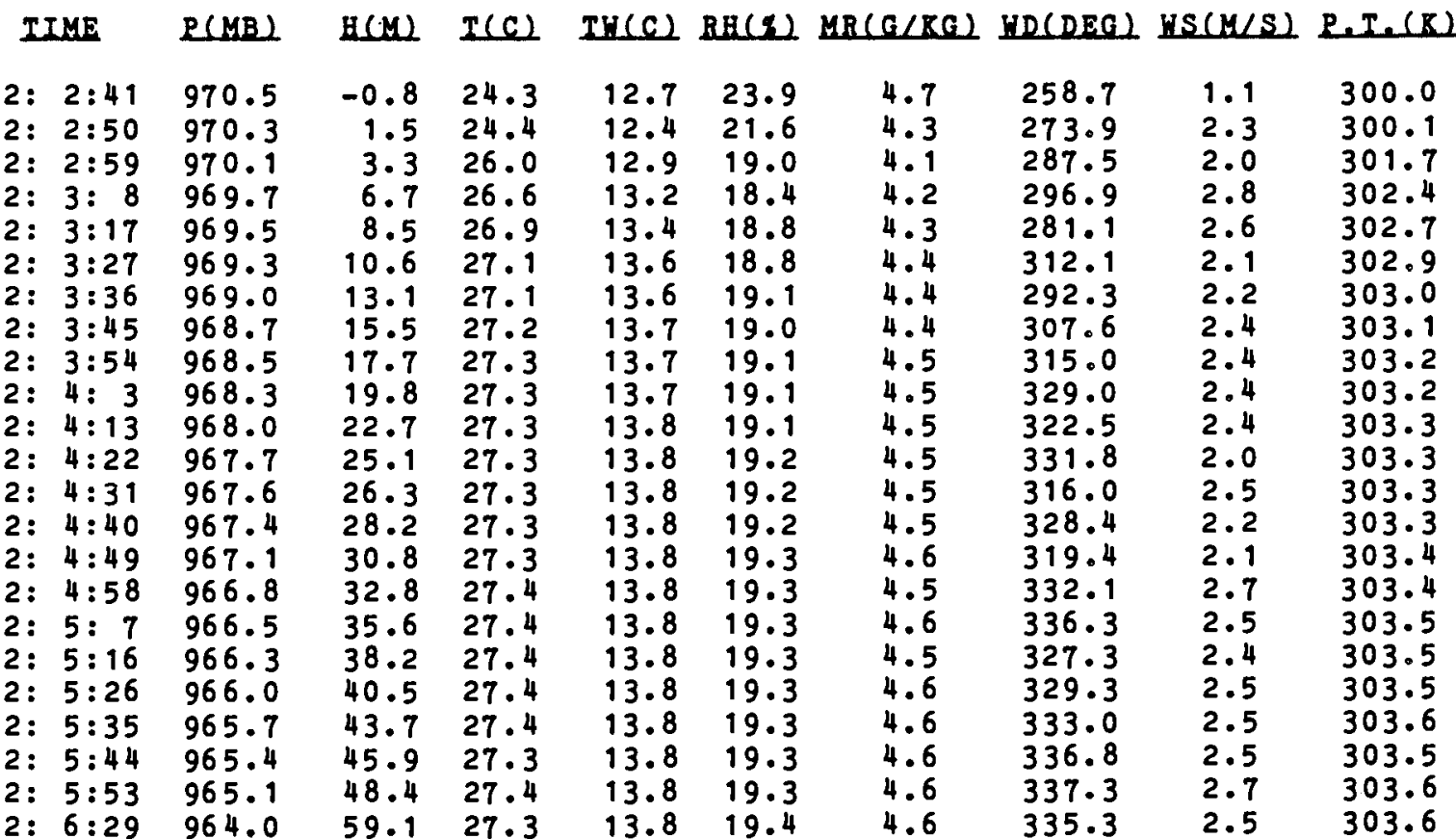




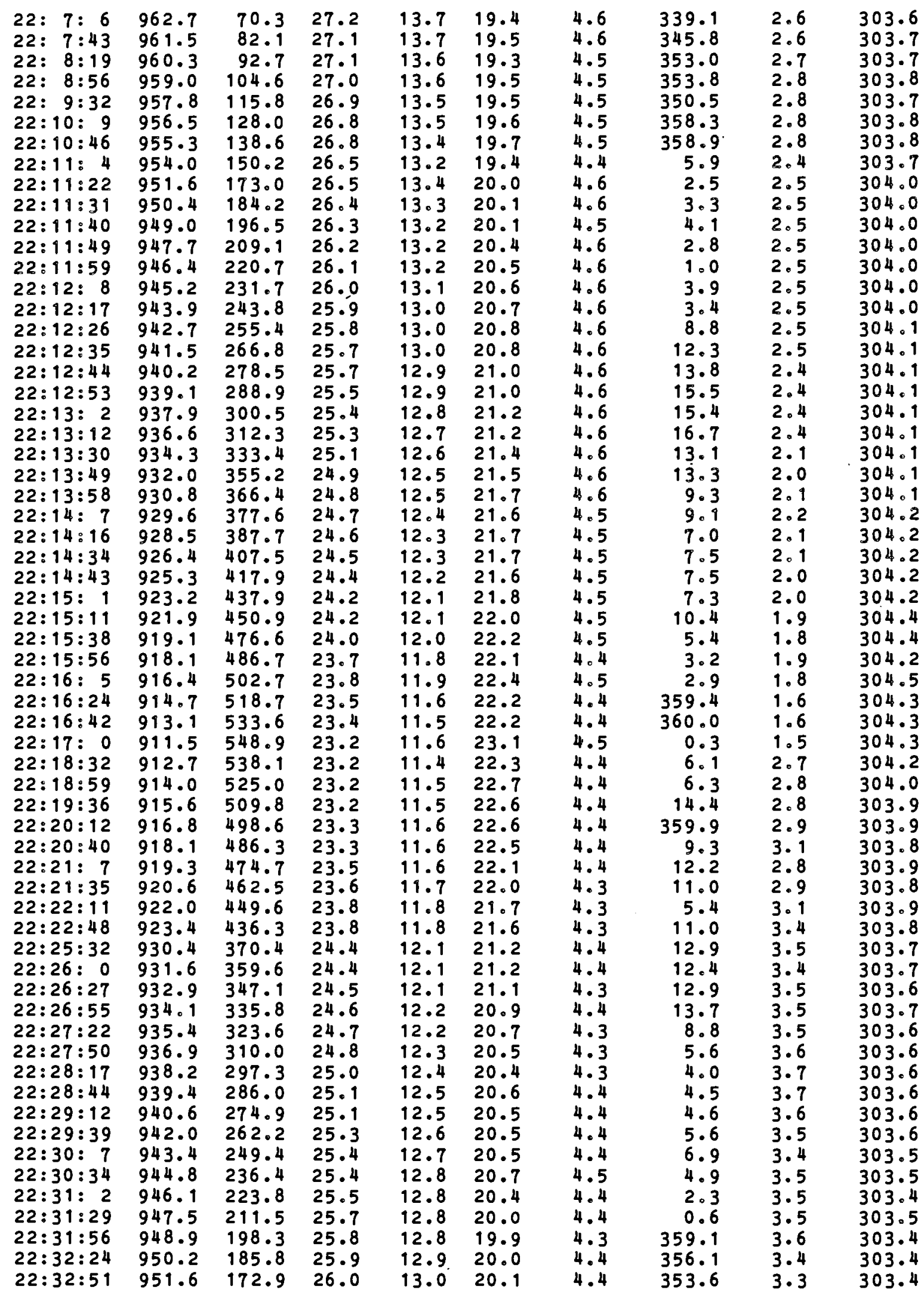




$\begin{array}{llllllllll}22: 33: 19 & 953.2 & 158.9 & 26.1 & 13.1 & 19.8 & 4.4 & 349.5 & 3.4 & 303.4 \\ 22: 33: 46 & 954.3 & 148.2 & 26.3 & 13.2 & 19.8 & 4.4 & 349.9 & 3.4 & 303.5 \\ 22: 34: 4 & 955.4 & 138.1 & 26.3 & 13.1 & 19.6 & 4.4 & 348.2 & 3.4 & 303.4 \\ 22: 34: 32 & 956.9 & 124.3 & 26.4 & 13.3 & 19.7 & 4.5 & 344.2 & 3.3 & 303.4 \\ 22: 34: 59 & 958.3 & 111.9 & 26.5 & 13.3 & 19.7 & 4.5 & 341.2 & 3.4 & 303.4 \\ 22: 35: 26 & 959.7 & 98.7 & 26.6 & 13.4 & 19.9 & 4.5 & 339.3 & 3.5 & 303.3 \\ 22: 35: 54 & 961.1 & 86.3 & 26.8 & 13.5 & 19.9 & 4.6 & 337.9 & 3.6 & 303.3 \\ 22: 36: 22 & 962.5 & 72.6 & 26.8 & 13.6 & 19.9 & 4.6 & 335.2 & 3.6 & 303.3 \\ 22: 36: 49 & 964.0 & 59.7 & 26.9 & 13.7 & 19.9 & 4.6 & 328.1 & 3.3 & 303.3 \\ 22: 37: 16 & 965.4 & 46.2 & 27.0 & 13.7 & 19.9 & 4.6 & 327.4 & 3.2 & 303.2 \\ 22: 37: 26 & 965.9 & 42.0 & 27.0 & 13.7 & 19.9 & 4.6 & 324.9 & 3.0 & 303.2 \\ 22: 37: 35 & 966.3 & 38.2 & 27.0 & 13.7 & 19.9 & 4.6 & 324.8 & 3.0 & 303.1 \\ 22: 37: 44 & 966.5 & 36.2 & 27.1 & 13.8 & 19.9 & 4.6 & 323.0 & 3.0 & 303.2 \\ 22: 37: 53 & 967.0 & 32.5 & 27.1 & 13.7 & 19.8 & 4.6 & 325.9 & 2.8 & 303.1 \\ 22: 38: 2 & 967.4 & 28.4 & 27.1 & 13.8 & 19.8 & 4.6 & 326.6 & 2.7 & 303.1 \\ 22: 38: 11 & 967.8 & 24.7 & 27.0 & 13.8 & 20.0 & 4.6 & 321.9 & 2.8 & 303.0 \\ 22: 38: 20 & 968.3 & 20.2 & 27.0 & 13.8 & 20.0 & 4.6 & 332.5 & 2.9 & 303.0 \\ 22: 38: 29 & 968.7 & 16.5 & 27.0 & 13.7 & 19.9 & 4.6 & 286.8 & 2.8 & 302.9 \\ 22: 38: 38 & 969.2 & 12.1 & 27.0 & 13.8 & 20.0 & 4.6 & 301.0 & 2.9 & 302.9 \\ 22: 38: 48 & 969.5 & 9.2 & 27.0 & 13.8 & 20.0 & 4.6 & 302.2 & 3.1 & 302.8 \\ 22: 38: 57 & 970.1 & 4.2 & 27.1 & 13.8 & 20.2 & 4.7 & 293.8 & 2.3 & 302.8 \\ 22: 39: 6 & 970.5 & -0.1 & 26.2 & 13.8 & 22.6 & 5.0 & 263.3 & 1.7 & 301.9 \\ 22: 39: 15 & 970.6 & -1.1 & 24.8 & 13.5 & 25.7 & 5.2 & 260.2 & 1.2 & 300.5 \\ 22: 39: 25 & 970.6 & -0.6 & 24.0 & 13.1 & 26.7 & 5.1 & 252.5 & 1.3 & 299.7 \\ 22: 39: 34 & 970.6 & -0.3 & 23.9 & 12.9 & 26.4 & 5.1 & 254.6 & 1.2 & 299.6 \\ 22: 39: 43 & 970.5 & 0.4 & 23.7 & 12.7 & 25.9 & 4.9 & 261.4 & 1.6 & 299.4 \\ 22: 39: 52 & 970.5 & 0.4 & 23.7 & 12.7 & 25.5 & 4.8 & 267.0 & 1.4 & 299.4 \\ 22: 40: 1 & 970.6 & -1.1 & 23.8 & 12.6 & 24.8 & 4.7 & 260.8 & 1.1 & 299.5 \\ 22: 40: 10 & 970.5 & 0.0 & 23.8 & 12.7 & 25.6 & 4.9 & 260.1 & 0.9 & 299.5 \\ 22: 40: 19 & 970.6 & -0.5 & 23.6 & 12.6 & 25.6 & 4.8 & 248.8 & 1.0 & 299.3 \\ 22: 40: 28 & 970.5 & 0.2 & 23.6 & 12.7 & 25.7 & 4.9 & 256.6 & 1.4 & 299.3\end{array}$

Iuly 2-3, 1981: 2331-2353 PST

IIME

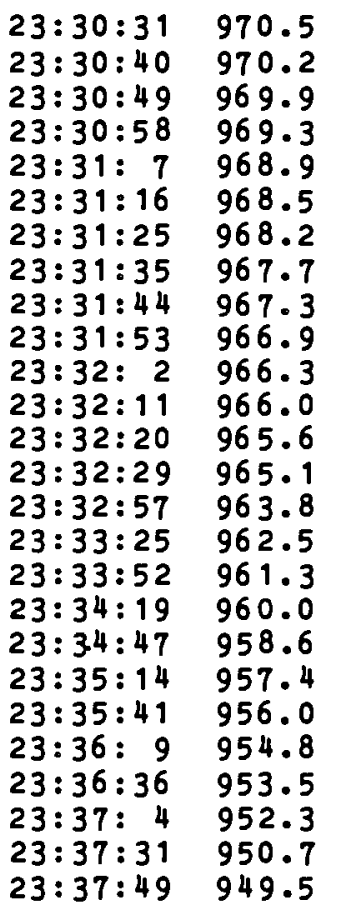

$H(H) \quad I(C)$

$\begin{array}{rr}0.7 & 22.7 \\ 4.0 & 24.9 \\ 6.9 & 25.8 \\ 12.1 & 26.0 \\ 15.9 & 26.3 \\ 19.0 & 26.4 \\ 22.4 & 26.4 \\ 26.7 & 26.4 \\ 29.9 & 26.4 \\ 34.0 & 26.4 \\ 38.9 & 26.4 \\ 42.3 & 26.4 \\ 45.8 & 26.4 \\ 49.9 & 26.4 \\ 61.8 & 26.4 \\ 74.3 & 26.3 \\ 85.1 & 26.2 \\ 97.0 & 26.1 \\ 109.5 & 26.0 \\ 120.9 & 25.9 \\ 133.5 & 25.8 \\ 144.3 & 25.7 \\ 156.5 & 25.5 \\ 167.2 & 25.4 \\ 181.7 & 25.3 \\ 192.9 & 25.2\end{array}$

$I H(c)$ RH(C)

$\begin{array}{ll}11.6 & 23.5 \\ 12.3 & 19.4 \\ 12.8 & 19.2 \\ 13.1 & 19.7 \\ 13.2 & 19.8 \\ 13.4 & 20.1 \\ 13.4 & 20.2 \\ 13.4 & 20.2 \\ 13.4 & 20.2 \\ 13.4 & 20.4 \\ 13.4 & 20.3 \\ 13.4 & 20.5 \\ 13.5 & 20.5 \\ 13.5 & 20.5 \\ 13.5 & 20.7 \\ 13.5 & 21.2 \\ 13.5 & 21.2 \\ 13.4 & 21.4 \\ 13.4 & 21.4 \\ 13.3 & 21.4 \\ 13.2 & 21.6 \\ 13.2 & 21.7 \\ 13.1 & 22.0 \\ 13.1 & 22.2 \\ 13.1 & 22.4 \\ 13.0 & 22.5\end{array}$

4.2
4.0
$4 \cdot 1$
$4 \cdot 3$
4.4
$4 \cdot 5$
4.5
$4 \cdot 5$
4.5
4.5
4.5
4.6
4.6
4.6
4.6
4.7
4.7
4.7
4.7
4.7
4.7
4.7
4.7
4.7
4.8
4.8

286.5
301.7
304.1
313.0
310.6
306.1
313.4
343.0
346.7
337.6
355.9
353.9
357.8
0.1
9.0
6.8
11.9
10.3
6.7
5.4
10.5
13.0
12.4
12.7
8.5
6.6

$\begin{array}{ll}1.6 & 298.4 \\ 1.4 & 300.7 \\ 1.1 & 301.6 \\ 1.0 & 301.9 \\ 1.2 & 302.1 \\ 1.7 & 302.3 \\ 1.6 & 302.3 \\ 1.4 & 302.3 \\ 1.6 & 302.4 \\ 1.8 & 302.4 \\ 1.7 & 302.5 \\ 1.7 & 302.5 \\ 1.7 & 302.6 \\ 1.9 & 302.6 \\ 2.2 & 302.7 \\ 2.1 & 302.8 \\ 2.3 & 302.7 \\ 2.3 & 302.7 \\ 2.2 & 302.8 \\ 2.2 & 302.8 \\ 2.4 & 302.8 \\ 2.3 & 302.8 \\ 2.6 & 302.8 \\ 2.5 & 302.8 \\ 2.3 & 302.8 \\ 2.5 & 302.8\end{array}$




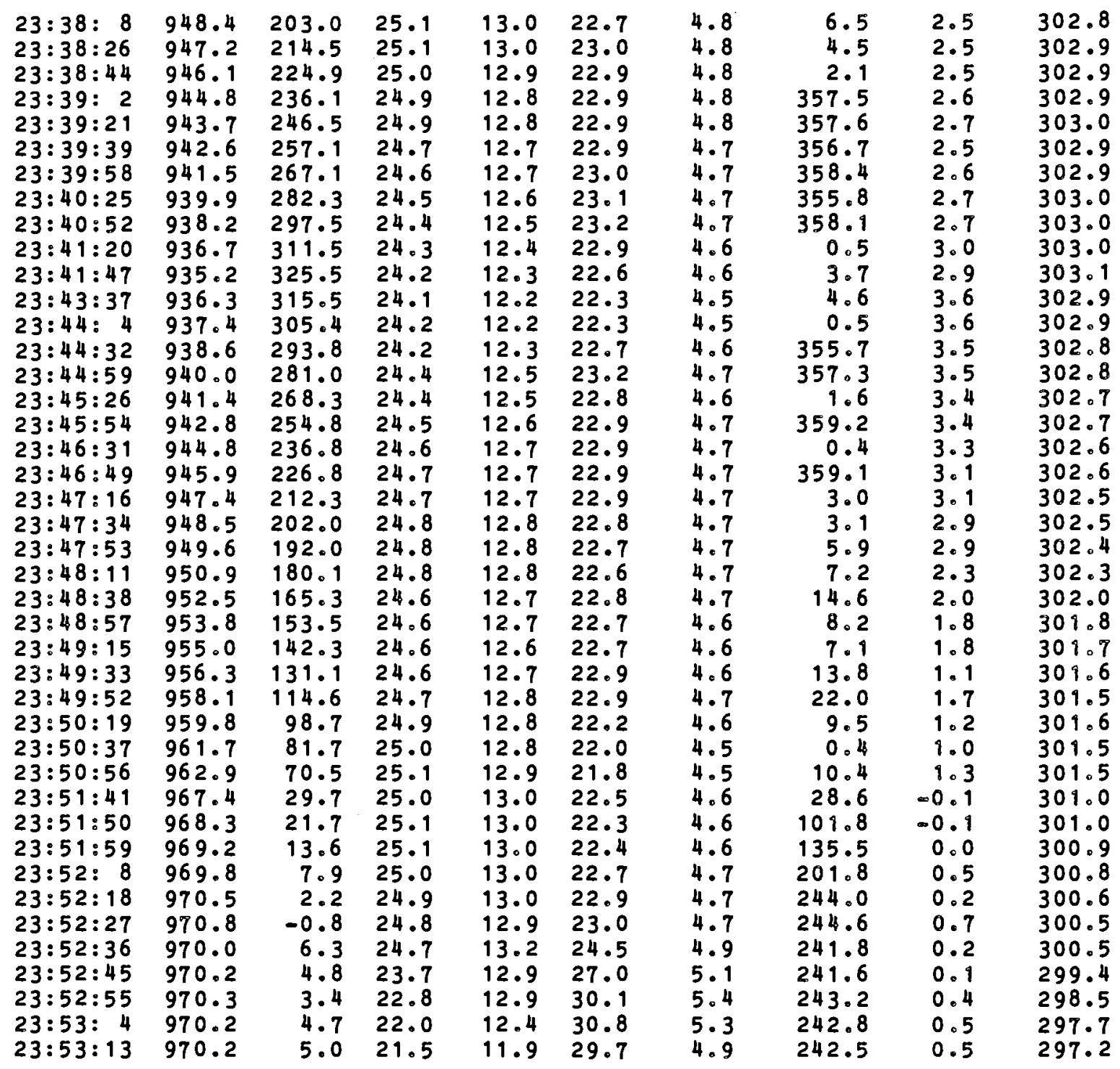

IU17 2-3. 1981; 2358-0025 RST

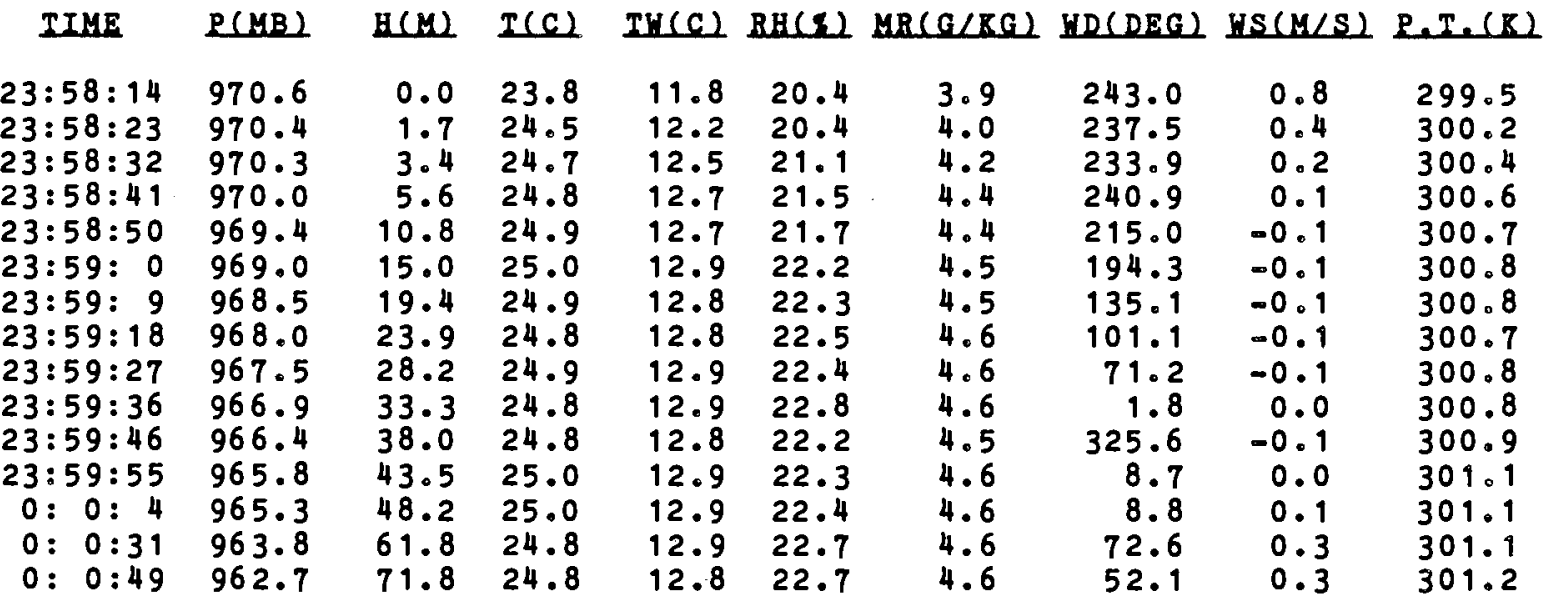




\begin{tabular}{|c|c|c|c|c|c|c|c|c|c|c|}
\hline $\begin{array}{l}0: \\
0:\end{array}$ & $\begin{array}{l}1: 17 \\
1: 44\end{array}$ & $\begin{array}{l}961.3 \\
959.9\end{array}$ & $\begin{array}{l}84.8 \\
97.0\end{array}$ & $\begin{array}{l}24.7 \\
24.6\end{array}$ & $\begin{array}{l}12.8 \\
12.8\end{array}$ & $\begin{array}{l}22.9 \\
23.1\end{array}$ & $\begin{array}{l}4.7 \\
4.7\end{array}$ & $\begin{array}{l}58.1 \\
57.3\end{array}$ & $\begin{array}{l}0.3 \\
0.6\end{array}$ & $\begin{array}{l}301.2 \\
301.2\end{array}$ \\
\hline 0: & $2: 11$ & 958.4 & 110.8 & 24.5 & 12.7 & 23.1 & 4.7 & 45.7 & 0.5 & 301.3 \\
\hline $0:$ & $2: 39$ & 957.0 & 123.3 & 24.5 & 12.7 & 23.1 & 4.6 & 33.9 & 0.5 & 301.4 \\
\hline 0 & $3: 7$ & 955.6 & 136.7 & 24.4 & 12.6 & 23.3 & 4.7 & 26.4 & 0.4 & 301.4 \\
\hline $0:$ & $3: 34$ & 954.2 & 149.1 & 24.4 & 12.6 & 23.3 & 4.7 & 22.2 & 0.5 & 301.5 \\
\hline $0:$ & $4: 1$ & 952.8 & 162.0 & $24 \cdot 3$ & 12.6 & $\begin{array}{r}23.5 \\
? 2\end{array}$ & 4.7 & $\begin{array}{l}18.5 \\
23.4\end{array}$ & 0.5 & $\begin{array}{l}301.6 \\
301.7\end{array}$ \\
\hline 0: & $\begin{array}{l}4: 29 \\
5: 5\end{array}$ & 949.9 & 188.7 & 24.5 & 12.6 & 23.1 & 4.7 & $\begin{array}{r}25.4 \\
9.7\end{array}$ & 2.5 & 302.0 \\
\hline $0:$ & $5: 33$ & 948.7 & 199.7 & 24.6 & 12.8 & 23.7 & 4.9 & 11.9 & 2.8 & 302.3 \\
\hline 0 : & $6: 0$ & 947.3 & 212.7 & 24.6 & 12.8 & 23.8 & 4.9 & 8.7 & 2.7 & 302.4 \\
\hline $\begin{array}{l}\text { 0: } \\
0:\end{array}$ & $\begin{array}{l}6: 28 \\
6: 55\end{array}$ & 946.0 & 224.8 & 24.5 & 12.7 & 23.6 & 4.8 & 2.1 & 2.6 & $\begin{array}{l}302.4 \\
302.4\end{array}$ \\
\hline 0 : & $7: 23$ & $\begin{array}{l}944.0 \\
943.5\end{array}$ & $\begin{array}{l}237.4 \\
248.1\end{array}$ & $\begin{array}{l}24.4 \\
24.3\end{array}$ & $\begin{array}{l}12.1 \\
12.6\end{array}$ & $\begin{array}{l}23.8 \\
23.9\end{array}$ & $\begin{array}{l}4.0 \\
4.8\end{array}$ & 0.2 & $\begin{array}{l}2.0 \\
2.8\end{array}$ & 302.5 \\
\hline 0 : & $7: 50$ & 942.3 & 258.7 & 24.2 & 12.6 & 24.0 & 4.8 & 357.1 & 2.8 & 302.5 \\
\hline $0:$ & $8: 17$ & 941.0 & 270.4 & 24.2 & 12.6 & 24.2 & 4.9 & 0.7 & 2.8 & 302.6 \\
\hline 0 : & $8: 45$ & 939.8 & 281.9 & 24.2 & 12.6 & 24.3 & 4.9 & 358.7 & 2.8 & 302.7 \\
\hline 0 : & $9: 12$ & 938.6 & 293.0 & 24.0 & 12.5 & 24.3 & 4.8 & 355.1 & 2.8 & 302.6 \\
\hline 0: & $9: 40$ & 937.4 & 304.0 & 24.0 & 12.5 & $24 \cdot 3$ & 4.8 & 353.0 & 3.0 & $\begin{array}{l}302.7 \\
302.5\end{array}$ \\
\hline $\begin{array}{l}0: \\
0:\end{array}$ & $\begin{array}{l}12: 6 \\
12: 43\end{array}$ & $\begin{array}{l}938.7 \\
939.9\end{array}$ & $\begin{array}{l}292.5 \\
280.8\end{array}$ & $\begin{array}{l}23.9 \\
24.0\end{array}$ & $\begin{array}{l}12.4 \\
12.4\end{array}$ & $\begin{array}{l}24.1 \\
24.0\end{array}$ & $\begin{array}{l}4.8 \\
4.8\end{array}$ & $\begin{array}{r}359.9 \\
2.4\end{array}$ & $\begin{array}{l}3.4 \\
3.3\end{array}$ & $\begin{array}{l}302.5 \\
302.5\end{array}$ \\
\hline $0:$ & $13: 19$ & 941.1 & 269.5 & 24.0 & 12.5 & 24.1 & 4.8 & 5.9 & 3.1 & 302.4 \\
\hline $0:$ & $13: 56$ & 942.3 & 258.4 & 24.1 & 12.5 & 23.9 & 4.8 & 2.4 & 3.1 & 302.4 \\
\hline 0 : & $14: 32$ & 943.6 & 246.8 & 24.2 & 12.6 & 23.9 & 4.8 & 4.5 & 3.1 & 302.4 \\
\hline 0 : & 15: 9 & 944.9 & 234.6 & 24.3 & 12.6 & 23.8 & 4.8 & 6.6 & 3.0 & 302.3 \\
\hline $0:$ & $15: 36$ & 946.1 & 223.7 & 24.3 & 12.6 & 23.7 & 4.8 & 11.5 & 2.8 & 302.2 \\
\hline 0 & $16: 13$ & 947.6 & 209.6 & 24.3 & 12.6 & 23.8 & 4.8 & 18.7 & 2.8 & 302.0 \\
\hline 0 & $16: 40$ & 948.8 & 198.6 & 24.2 & 12.5 & 23.6 & 4.7 & 21.2 & 2.6 & 301.8 \\
\hline 0 & $17: 8$ & 950.3 & 185.4 & 24.1 & 12.6 & 24.4 & 4.8 & 25.0 & 2.6 & 301.6 \\
\hline & $17: 35$ & 951.8 & 171.1 & 24.1 & 12.6 & 24.3 & 4.8 & 27.8 & 2.3 & 301.4 \\
\hline & $17: 53$ & 952.9 & 161.1 & 24.0 & 12.6 & 24.5 & 4.8 & 25.7 & 2.0 & 301.3 \\
\hline 0 & $18: 20$ & 954.6 & 145.5 & 24.0 & 12.6 & 24.7 & 4.8 & 24.9 & 1.4 & 301.1 \\
\hline 0 & $18: 39$ & 955.8 & 134.2 & 24.0 & 12.7 & 24.9 & 4.9 & 20. & 0.7 & 301.0 \\
\hline & $18: 57$ & 957.4 & 119.7 & 24.0 & 12.6 & 24.4 & 4. & 31.7 & 1.5 & 300.9 \\
\hline 0 & $19: 25$ & 959.0 & 105.0 & 24.3 & 12.8 & $24 \cdot 3$ & 4. & 15. & & 301.0 \\
\hline & $19: 43$ & 960.9 & 88.2 & 24.4 & 12.8 & 24.0 & 4.8 & 5.9 & 0.6 & 300.9 \\
\hline & $20: 1$ & 962.3 & 75.9 & 24.4 & 12.8 & 24.1 & 4.8 & & 0.5 & 300.9 \\
\hline & $20: 38$ & 963.9 & 61.2 & 24.6 & 12.9 & 23.7 & 4.8 & 23.3 & 0.1 & 300.9 \\
\hline & $21: 5$ & 965.2 & 49.6 & 24.7 & 13.0 & 23. & 4. & & -0.1 & 300.9 \\
\hline & $21: 14$ & 965.6 & 45.8 & 24.7 & 13.0 & 23.8 & 4.8 & 10.4 & -0.1 & 300.8 \\
\hline & $21: 23$ & 965.9 & 42.8 & 24.6 & 13.0 & 24. & 4. & 304.9 & -0.1 & 300.7 \\
\hline & $21: 32$ & 966.3 & 39.6 & 24.4 & 12.8 & 24.0 & 4.8 & 278.4 & 0.0 & 300.5 \\
\hline & $21: 42$ & 966.6 & 36.6 & 24.6 & 12.9 & 23. & 4.7 & 246.9 & -0.1 & 300.7 \\
\hline & $21: 51$ & 966.9 & 33.5 & 24.6 & 12.9 & 23.4 & 4.7 & 244.1 & -0.1 & 300.7 \\
\hline & $22: 0$ & & 30. & 24.7 & 13.0 & 23 & 4. & 244. & & 300.7 \\
\hline & 22: 9 & & 27.6 & 24.7 & 12.9 & 23. & 4.7 & 243.3 & 0.0 & 300.7 \\
\hline & $22: 19$ & & 25.4 & 24.7 & 13.0 & & 4.7 & 239. & 0.2 & \\
\hline & $22: 28$ & 96 & 23.5 & 24.7 & 12.9 & 23. & 4. & 232. & 0.3 & 300.6 \\
\hline & $22: 37$ & 968 & 21.6 & 24.8 & 13.0 & & 4. & 229 & 0.4 & 300.7 \\
\hline & $22: 46$ & & 18. & 24.8 & 13.0 & & 4.7 & $226 。$ & 0.3 & 300.6 \\
\hline & $22: 55$ & & 16. & 24.8 & 13.0 & & 4.7 & 230. & 0.5 & 300.7 \\
\hline & $23: 4$ & & 16. & 24.8 & 13.0 & 23 & 4.7 & 244.0 & 0.4 & 300.7 \\
\hline & $23: 13$ & $96 \mathrm{~s}$ & 14. & 24.9 & 13.0 & & 4.7 & 244.1 & 0.6 & 300.7 \\
\hline & $23: 22$ & & 13. & 24.8 & 13.0 & 23. & 4.7 & 244.1 & & 300.7 \\
\hline 0 & $23: 31$ & 969 & & 24.8 & 13.0 & 23. & 4.7 & 244.1 & 0.6 & 300.6 \\
\hline $0:$ & $23: 41$ & & 10 & 24.9 & 13.0 & & 4.7 & 244.2 & & 300.6 \\
\hline $0:$ & $23: 50$ & 969. & 7.1 & 24.9 & 13.0 & 23. & 4.7 & 244.1 & 0.6 & 300.7 \\
\hline $0:$ & $23: 59$ & 970 & 6.2 & 24.9 & 13.1 & & 4.7 & 244.2 & 0.8 & \\
\hline & $24: 8$ & 970.2 & 4.1 & 25.0 & 13.1 & 23. & 4.7 & 244.2 & 0.7 & 300.7 \\
\hline & $24: 17$ & & 3.5 & 25.0 & 13.1 & 23.2 & 4.7 & 243.4 & 1.1 & 300.7 \\
\hline 0 & $24: 26$ & 970.3 & 2.9 & 24.9 & 13.0 & 23.1 & 4.7 & 244.1 & 1.3 & 300.6 \\
\hline $0:$ & $24: 35$ & 970.4 & 2.3 & 24.9 & 13.0 & 23.1 & 4.7 & 241.6 & 1.3 & 300.6 \\
\hline
\end{tabular}




$\begin{array}{lrrrrrrrrr}0: 24: 44 & 970.6 & 0.2 & 24.9 & 13.0 & 23.3 & 4.7 & 244.1 & 1.7 & 300.6 \\ 0: 24: 54 & 970.9 & -2.1 & 24.8 & 13.0 & 23.7 & 4.8 & 243.9 & 2.3 & 300.4 \\ 0: 25: 3 & 971.0 & -3.6 & 23.2 & 12.9 & 29.2 & 5.3 & 244.1 & 1.5 & 298.8 \\ 0: 25: 12 & 970.6 & 0.4 & 21.5 & 12.3 & 32.7 & 5.4 & 244.1 & -0.2 & 297.1 \\ 0: 25: 21 & 970.5 & 1.0 & 20.7 & 11.9 & 33.9 & 5.3 & 244.0 & 0.2 & 296.3 \\ 0: 25: 31 & 970.0 & 5.8 & 20.4 & 11.6 & 33.0 & 5.1 & 244.0 & 0.2 & 296.1 \\ 0: 25: 40 & 970.0 & 5.8 & 20.1 & 11.3 & 32.5 & 5.0 & 244.8 & 0.5 & 295.9 \\ 0: 25: 49 & 968.8 & 16.4 & 20.2 & 11.3 & 32.5 & 5.0 & 315.4 & 0.8 & 296.0 \\ 0: 25: 58 & 970.2 & 4.0 & 20.1 & 11.3 & 32.4 & 4.9 & 320.1 & 0.5 & 295.8\end{array}$

Iuly 2-3. 1981: 0036-0114 RST

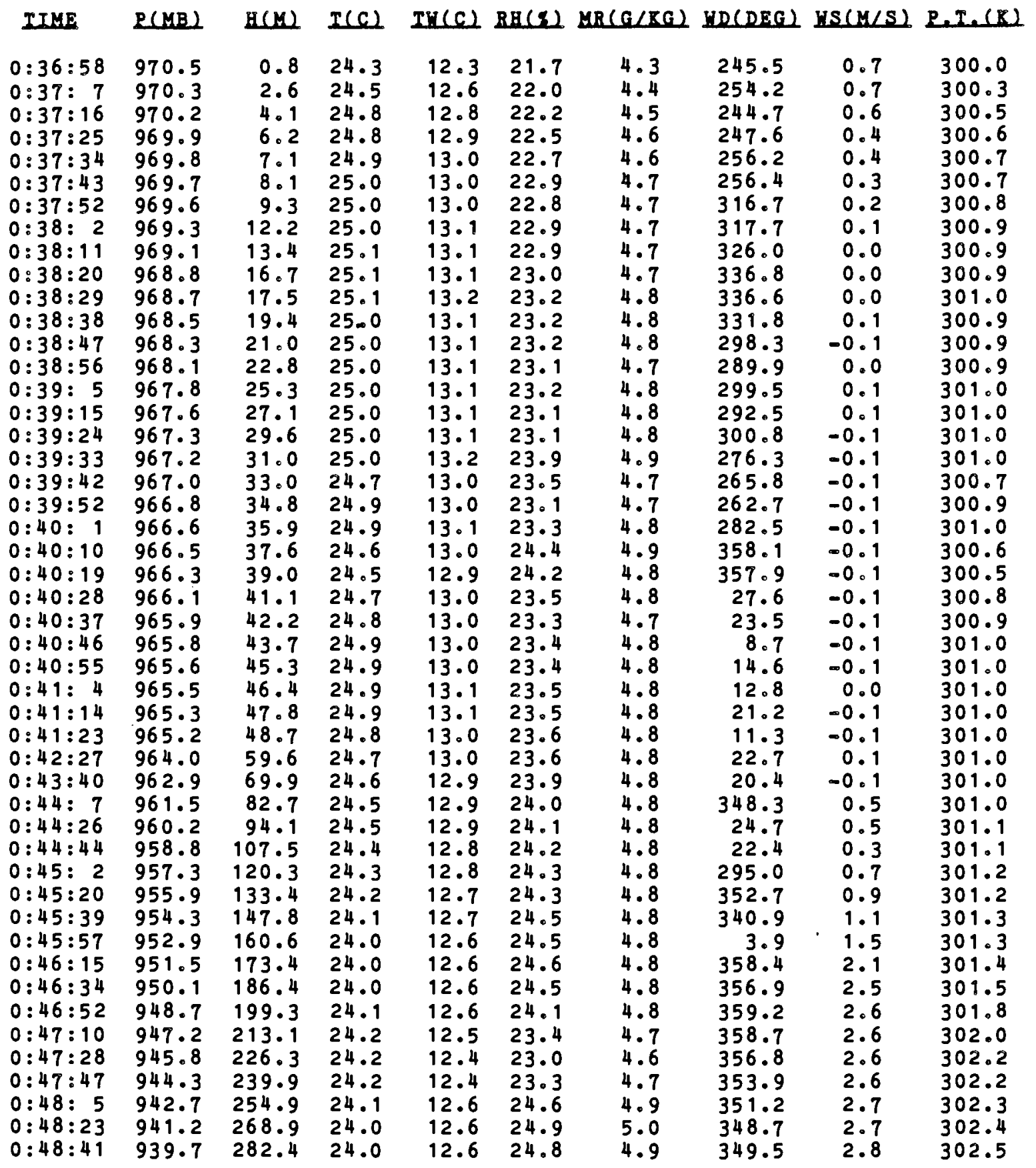




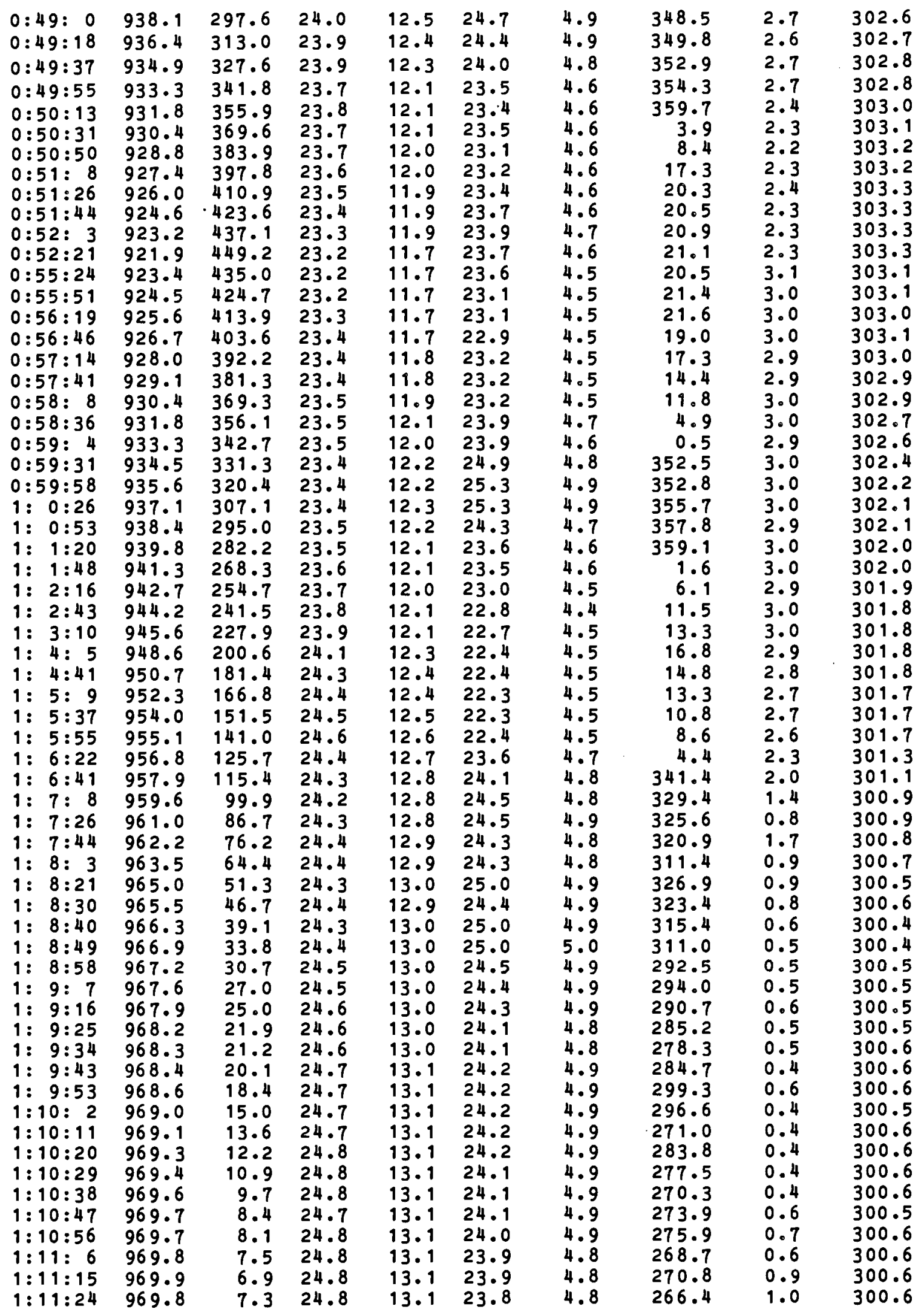




$\begin{array}{llllllllll}1: 11: 33 & 970.1 & 5.1 & 24.9 & 13.1 & 23.8 & 4.8 & 262.0 & 1.0 & 300.6 \\ 1: 11: 43 & 970.1 & 5.2 & 24.9 & 13.2 & 23.8 & 4.8 & 261.2 & 0.9 & 300.7 \\ 1: 11: 52 & 970.3 & 3.2 & 24.9 & 13.2 & 23.9 & 4.9 & 257.7 & 0.9 & 300.6 \\ 1: 12: 1 & 970.3 & 2.9 & 24.9 & 13.2 & 24.1 & 4.9 & 261.8 & 1.6 & 300.6 \\ 1: 12: 10 & 970.2 & 3.8 & 24.8 & 13.2 & 24.2 & 4.9 & 265.7 & 1.5 & 300.6 \\ 1: 12: 19 & 970.4 & 2.1 & 24.7 & 13.2 & 24.6 & 4.9 & 266.4 & 1.2 & 300.4 \\ 1: 12: 28 & 970.4 & 2.0 & 24.6 & 13.1 & 24.6 & 4.9 & 266.1 & 1.3 & 300.4 \\ 1: 12: 37 & 970.5 & 1.5 & 24.6 & 13.1 & 24.5 & 4.9 & 276.4 & 1.4 & 300.3 \\ 1: 12: 46 & 970.6 & 0.7 & 24.6 & 13.1 & 24.3 & 4.9 & 276.6 & 1.6 & 300.3 \\ 1: 12: 55 & 970.6 & 0.1 & 24.4 & 13.1 & 25.5 & 5.0 & 263.0 & 1.9 & 300.1 \\ 1: 13: 5 & 970.7 & -0.5 & 24.0 & 13.0 & 26.0 & 5.0 & 259.8 & 2.0 & 299.7 \\ 1: 13: 14 & 970.8 & -1.5 & 22.8 & 12.8 & 29.8 & 5.4 & 255.4 & 1.6 & 298.5 \\ 1: 13: 23 & 970.9 & -2.0 & 21.9 & 12.5 & 32.2 & 5.4 & 248.3 & 0.9 & 297.5 \\ 1: 13: 32 & 970.8 & -1.5 & 21.0 & 12.0 & 32.7 & 5.3 & 248.9 & 0.8 & 296.7 \\ 1: 13: 41 & 970.8 & -1.6 & 20.8 & 11.8 & 32.4 & 5.2 & 248.0 & 1.0 & 296.5 \\ 1: 13: 50 & 970.9 & -2.3 & 20.6 & 11.6 & 32.4 & 5.1 & 249.7 & 0.9 & 296.3 \\ 1: 13: 59 & 970.9 & -2.1 & 20.6 & 11.6 & 32.5 & 5.1 & 249.6 & 1.1 & 296.2 \\ 1: 14: 8 & 970.7 & -0.3 & 20.7 & 11.7 & 32.6 & 5.1 & 253.3 & 1.0 & 296.4 \\ 1: 14: 18 & 970.8 & -1.4 & 20.6 & 11.6 & 32.0 & 5.0 & 248.4 & 0.8 & 296.2 \\ 1: 14: 27 & 970.8 & -1.2 & 20.6 & 11.7 & 32.4 & 5.1 & 250.7 & 1.1 & 296.3\end{array}$

InIy 2-3, 1981: 0123-0140 PST

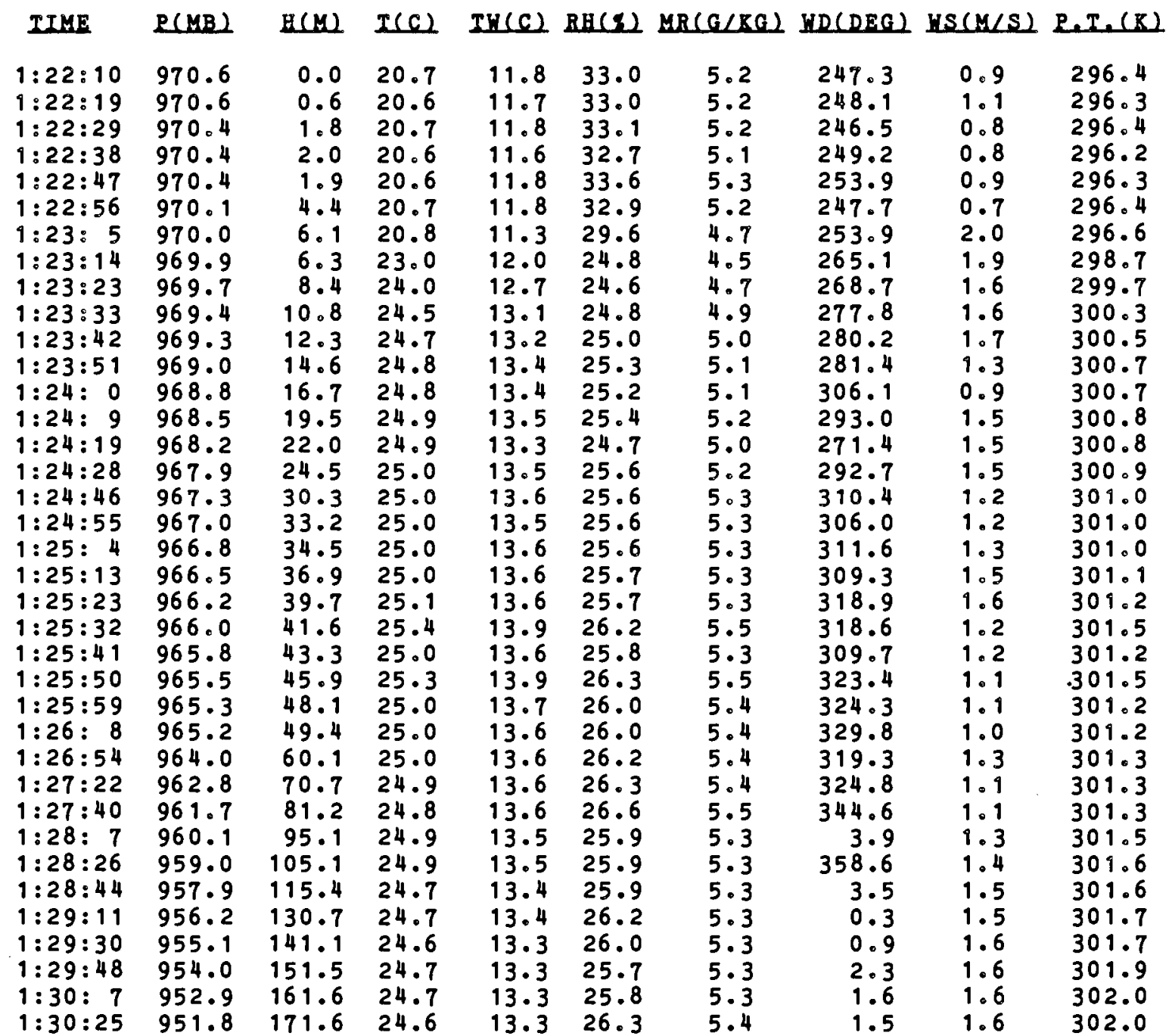




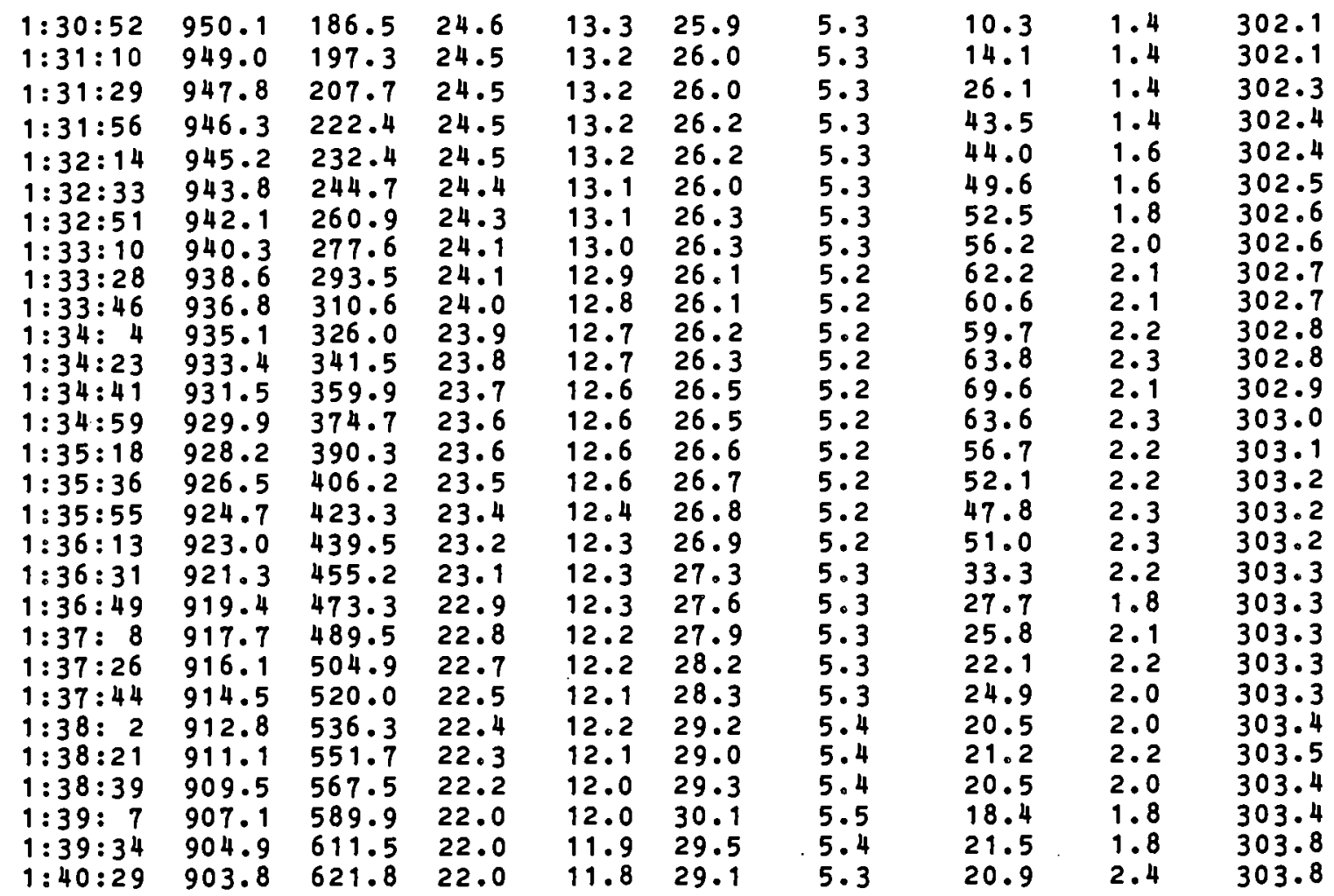

July 2-3, 1981: 0207-0234 RST

$\begin{array}{llllllllll}2: 7: 12 & 970.6 & 0.0 & 25.0 & 12.7 & 21.2 & 4.3 & 280.7 & 1.1 & 300.7 \\ 2: 7: 22 & 970.3 & 3.0 & 25.0 & 12.8 & 21.4 & 4.4 & 276.3 & 1.0 & 300.8 \\ 2: 7: 31 & 970.2 & 3.8 & 25.1 & 12.9 & 21.6 & 4.5 & 282.1 & 1.0 & 300.9 \\ 2: 7: 40 & 970.0 & 5.7 & 25.2 & 12.9 & 21.7 & 4.5 & 288.7 & 1.0 & 301.0 \\ 2: 7: 49 & 969.9 & 6.6 & 25.2 & 13.0 & 21.7 & 4.5 & 290.5 & 1.1 & 301.0 \\ 2: 7: 58 & 969.7 & 8.1 & 25.3 & 13.0 & 21.9 & 4.6 & 292.5 & 1.2 & 301.1 \\ 2: 8: 7 & 969.4 & 10.5 & 25.3 & 13.0 & 21.8 & 4.5 & 298.4 & 1.1 & 301.1 \\ 2: 8: 16 & 969.2 & 12.2 & 25.3 & 13.0 & 21.9 & 4.6 & 297.3 & 1.1 & 301.1 \\ 2: 8: 25 & 969.0 & 14.3 & 25.3 & 13.0 & 21.9 & 4.6 & 286.7 & 1.1 & 301.2 \\ 2: 8: 34 & 968.9 & 15.1 & 25.3 & 13.0 & 22.0 & 4.6 & 303.4 & 1.1 & 301.1 \\ 2: 8: 44 & 968.8 & 16.4 & 25.3 & 13.0 & 22.0 & 4.6 & 300.4 & 1.0 & 301.2 \\ 2: 8: 53 & 968.7 & 17.4 & 25.3 & 13.1 & 22.0 & 4.6 & 283.9 & 1.0 & 301.2 \\ 2: 9: 12 & 968.4 & 19.5 & 25.3 & 13.0 & 21.9 & 4.6 & 289.4 & 1.1 & 301.2 \\ 2: 9: 11 & 968.3 & 20.3 & 25.3 & 13.1 & 22.0 & 4.6 & 291.0 & 1.0 & 301.2 \\ 2: 9: 20 & 968.0 & 23.2 & 25.3 & 13.1 & 22.1 & 4.6 & 305.0 & 1.0 & 301.3 \\ 2: 9: 29 & 967.9 & 23.9 & 25.3 & 13.1 & 22.1 & 4.6 & 299.2 & 0.9 & 301.3 \\ 2: 9: 38 & 967.8 & 25.5 & 25.3 & 13.1 & 22.1 & 4.6 & 296.2 & 0.9 & 301.3 \\ 2: 9: 47 & 967.6 & 27.0 & 25.4 & 13.1 & 22.1 & 4.6 & 299.6 & 1.0 & 301.3 \\ 2: 9: 56 & 967.5 & 27.5 & 25.3 & 13.0 & 22.0 & 4.6 & 299.3 & 0.9 & 301.3 \\ 2: 10: 5 & 967.3 & 30.0 & 25.3 & 13.0 & 22.0 & 4.6 & 296.7 & 0.8 & 301.3 \\ 2: 10: 15 & 967.1 & 31.6 & 25.3 & 13.0 & 21.8 & 4.6 & 292.9 & 0.8 & 301.4 \\ 2: 10: 24 & 967.0 & 32.2 & 25.3 & 13.1 & 21.9 & 4.6 & 301.1 & 0.7 & 301.4 \\ 2: 10: 33 & 966.8 & 34.2 & 25.4 & 13.1 & 21.9 & 4.6 & 299.3 & 0.6 & 301.4 \\ 2: 10: 42 & 966.8 & 34.2 & 25.4 & 13.1 & 21.8 & 4.6 & 296.0 & 0.7 & 301.4 \\ 2: 10: 51 & 966.5 & 37.0 & 25.4 & 13.1 & 21.8 & 4.6 & 299.5 & 0.7 & 301.4 \\ 2: 11: 1 & 966.6 & 36.2 & 25.4 & 13.0 & 21.8 & 4.6 & 301.9 & 0.6 & 301.4 \\ 2: 11: 10 & 966.2 & 39.4 & 25.3 & 13.0 & 21.7 & 4.6 & 297.0 & 0.7 & 301.4 \\ 2: 11: 19 & 966.0 & 42.0 & 25.3 & 13.0 & 21.7 & 4.6 & 300.4 & 0.6 & 301.4\end{array}$




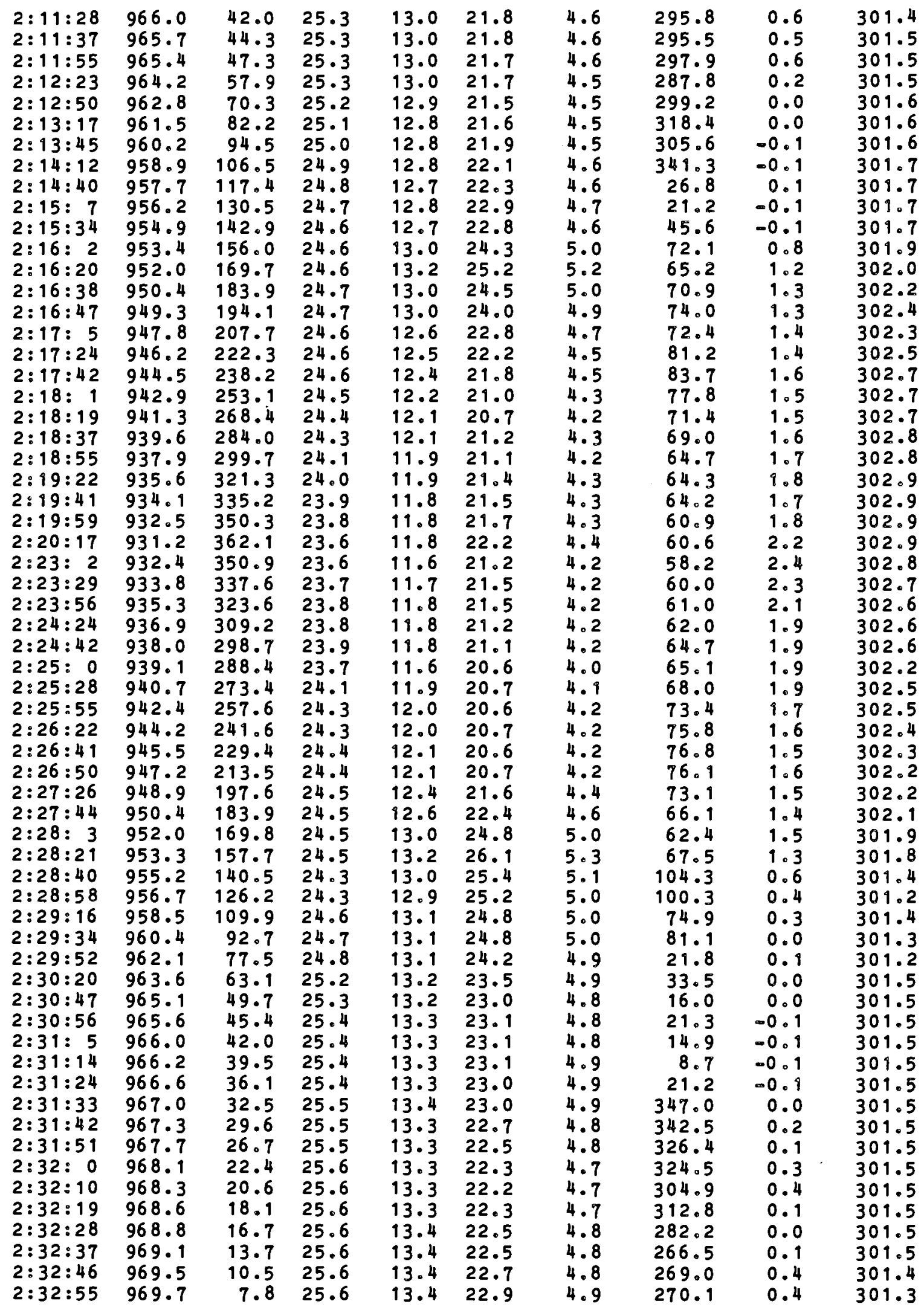




$\begin{array}{lrrrrrrrrr}2: 33: 4 & 969.9 & 6.2 & 25.5 & 13.5 & 23.2 & 4.9 & 250.8 & 0.7 & 301.3 \\ 2: 33: 13 & 970.1 & 4.7 & 25.5 & 13.5 & 23.5 & 5.0 & 244.3 & 1.0 & 301.3 \\ 2: 33: 32 & 970.6 & 0.4 & 24.0 & 13.5 & 28.9 & 5.6 & 243.9 & 1.9 & 299.7 \\ 2: 33: 41 & 969.7 & 8.0 & 22.4 & 13.0 & 32.9 & 5.8 & 244.5 & 0.0 & 298.1 \\ 2: 33: 50 & 970.1 & 4.4 & 21.3 & 12.5 & 34.6 & 5.7 & 244.1 & -0.1 & 297.0 \\ 2: 34: 8 & 970.6 & 0.3 & 20.9 & 12.0 & 33.3 & 5.3 & 244.5 & 0.0 & 296.6 \\ 2: 34: 17 & 970.6 & 0.4 & 20.8 & 11.9 & 33.2 & 5.3 & 244.2 & 0.0 & 296.5 \\ 2: 34: 26 & 970.8 & -1.2 & 20.8 & 11.9 & 32.9 & 5.2 & 244.1 & -0.1 & 296.4\end{array}$

Iuly 2-3. 1981: 0239-0303 RST

\begin{tabular}{|c|c|c|c|c|c|c|c|c|c|}
\hline IIME & $P(M B)$ & $H(M)$ & (c) & $L(c)$ & $a(s)$ & $R(G / R G)$ & $K D(D E G)$ & $\mathrm{HS}(\mathrm{K} / \mathrm{S})$ & P.T. \\
\hline $\begin{array}{l}2: 38: 40 \\
2: 38: 49 \\
2: 38: 58 \\
2: 39: 7 \\
2: 39: 16 \\
2: 39: 35 \\
2: 39: 44 \\
2: 39: 53 \\
2: 40: 2 \\
2: 40: 11 \\
2: 40: 20 \\
2: 40: 29 \\
2: 40: 38 \\
2: 40: 48 \\
2: 40: 57 \\
2: 41: 6 \\
2: 41: 24 \\
2: 41: 34 \\
2: 41: 43 \\
2: 41: 52 \\
2: 42: 1 \\
2: 42: 10 \\
2: 42: 19 \\
2: 42: 28 \\
2: 42: 37 \\
2: 42: 46 \\
2: 42: 56 \\
2: 43: 5 \\
2: 43: 14 \\
2: 43: 23 \\
2: 43: 32 \\
2: 43: 41 \\
2: 43: 50 \\
2: 43: 59 \\
2: 44: 8 \\
2: 44: 18 \\
2: 44: 27 \\
2: 45: 31 \\
2: 45: 49 \\
2: 46: 7 \\
2: 46: 26 \\
2: 46: 44 \\
2: 47: 2 \\
2: 47: 20 \\
2: 47: 38 \\
2: 47: 57 \\
2: 48: 15 \\
2: 48: 34 \\
2: 48: 52\end{array}$ & $\begin{array}{l}970.6 \\
970.0 \\
970.0 \\
969.7 \\
969.5 \\
969.2 \\
969.1 \\
969.0 \\
968.9 \\
968.8 \\
968.6 \\
968.4 \\
968.3 \\
968.2 \\
968.0 \\
967.9 \\
967.7 \\
967.5 \\
967.3 \\
967.3 \\
967.1\end{array}$ & $\begin{array}{r}-1.0 \\
4.9 \\
5.1 \\
7.5 \\
9.3 \\
12.0 \\
12.8 \\
13.8 \\
14.4 \\
15.9 \\
17.4 \\
18.8 \\
20.3 \\
20.9 \\
22.5 \\
23.3 \\
25.9 \\
27.7 \\
29.4 \\
29.2 \\
31.1 \\
32.2 \\
33.9 \\
34.8 \\
35.8 \\
37.3 \\
38.0 \\
38.0 \\
40.2 \\
41.8 \\
42.4 \\
44.2 \\
45.3 \\
46.1 \\
46.5 \\
48.5 \\
49.7 \\
200.7 \\
218.8 \\
237.7\end{array}$ & $\begin{array}{l}20 \cdot 0 \\
20 \cdot 1 \\
20 \cdot 0 \\
21 \cdot 4 \\
23 \cdot 2 \\
24 \cdot 5 \\
24 \cdot 7 \\
25 \cdot 0 \\
25 \cdot 1 \\
25 \cdot 2 \\
25 \cdot 3 \\
25 \cdot 3 \\
25 \cdot 3 \\
25 \cdot 3 \\
25 \cdot 3 \\
25 \cdot 3 \\
25 \cdot 3 \\
25 \cdot 4 \\
25 \cdot 4 \\
25 \cdot 3 \\
25 \cdot 4 \\
25 \cdot 3 \\
25 \cdot 3 \\
25 \cdot 4 \\
25 \cdot 3 \\
25 \cdot 3 \\
25 \cdot 3 \\
25 \cdot 3 \\
25 \cdot 3 \\
25 \cdot 3 \\
25 \cdot 3 \\
25 \cdot 3 \\
25 \cdot 3 \\
25 \cdot 3 \\
25 \cdot 3 \\
25 \cdot 2 \\
25 \cdot 2 \\
25 \cdot 1 \\
25 \cdot 1 \\
25 \cdot 0 \\
24 \cdot 9 \\
24 \cdot 8 \\
24 \cdot 6 \\
24 \cdot 5 \\
24 \cdot 3 \\
24 \cdot 1 \\
24 \cdot 0 \\
23 \cdot 8 \\
23 \cdot 8\end{array}$ & $\begin{array}{l}11.3 \\
11.4 \\
11.1 \\
10.9 \\
11.8 \\
12.6 \\
12.8 \\
13.0 \\
13.1 \\
13.2 \\
13.2 \\
13.3 \\
13.3 \\
13.3 \\
13.3 \\
13.3 \\
13.4 \\
13.4 \\
13.4 \\
13.5 \\
13.5 \\
13.5 \\
13.4 \\
13.4 \\
13.4 \\
13.3 \\
13.4 \\
13.4 \\
13.4 \\
13.4 \\
13.4 \\
13.4 \\
13.4 \\
13.4 \\
13.3 \\
13.3 \\
13.3 \\
13.4 \\
13.3 \\
13.3 \\
13.2 \\
13.2 \\
13.1 \\
13.0 \\
12.9 \\
12.9 \\
12.9 \\
12.9 \\
12.9\end{array}$ & $\begin{array}{l}33.2 \\
33.2 \\
31.7 \\
24.5 \\
22.6 \\
22.6 \\
22.5 \\
22.8 \\
22.7 \\
22.8 \\
23.0 \\
23.1 \\
23.2 \\
23.1 \\
23.3 \\
23.2 \\
23.5 \\
23.7 \\
23.7 \\
23.9 \\
24.0 \\
24.0 \\
23.9 \\
23.7 \\
23.4 \\
23.3 \\
23.5 \\
23.6 \\
23.6 \\
23.8 \\
23.7 \\
23.8 \\
23.8 \\
23.7 \\
23.7 \\
23.8 \\
23.8 \\
24.2 \\
24.2 \\
24.5 \\
24.4 \\
24.6 \\
24.9 \\
25.0 \\
25.1 \\
25 \cdot 7 \\
26.4 \\
26.8 \\
27.3\end{array}$ & $\begin{array}{l}5.0 \\
5.1 \\
4.8 \\
4.0 \\
4.2 \\
4.5 \\
4.5 \\
4.7 \\
4.7 \\
4.7 \\
4.8 \\
4.8 \\
4.9 \\
4.8 \\
4.9 \\
4.9 \\
4.9 \\
5.0 \\
5.0 \\
5.0 \\
5.0 \\
5.0 \\
5.0 \\
5.0 \\
4.9 \\
4.9 \\
4.9 \\
4.9 \\
4.9 \\
5.0 \\
5.0 \\
5.0 \\
5.0 \\
5.0 \\
4.9 \\
5.0 \\
4.9 \\
5.0 \\
5.0 \\
5.1 \\
5.0 \\
5.0 \\
5.1 \\
5.0 \\
5.0 \\
5.1 \\
5.2 \\
5.2 \\
5.3\end{array}$ & $\begin{array}{r}243.8 \\
243.9 \\
242.7 \\
244.0 \\
236.1 \\
244.0 \\
244.2 \\
244.2 \\
236.9 \\
210.6 \\
199.4 \\
182.3 \\
159.6 \\
136.9 \\
109.1 \\
92.8 \\
98.2 \\
93.6 \\
106.9 \\
90.8 \\
95.4\end{array}$ & $\begin{array}{r}0.0 \\
-0.1 \\
1.4 \\
1.7 \\
1.1 \\
0.8 \\
0.1 \\
0.2 \\
-0.1 \\
0.0 \\
-0.1 \\
-0.1 \\
0.0 \\
0.1 \\
0.0 \\
-0.1 \\
0.1 \\
0.2 \\
0.3 \\
0.2 \\
0.3 \\
0.2 \\
0.2 \\
0.4 \\
0.5 \\
0.4 \\
0.5 \\
0.6 \\
0.6 \\
0.4 \\
0.6 \\
0.6 \\
0.7 \\
0.7 \\
0.7 \\
0.6 \\
0.5 \\
0.9 \\
0.6 \\
0.6 \\
0.5 \\
0.7 \\
0.5 \\
0.6 \\
0.6 \\
0.6 \\
0.7 \\
0.7 \\
0.8\end{array}$ & $\begin{array}{l}295.7 \\
295.8 \\
295.7 \\
297.2 \\
299.0 \\
300.3 \\
300.6 \\
300.8 \\
300.9 \\
301.9 \\
301.1 \\
301.2 \\
301.2 \\
301.2 \\
301.2 \\
301.3 \\
301.3 \\
301.3 \\
301.4 \\
301.3 \\
301.4 \\
301.4 \\
301.4 \\
301.4 \\
301.4 \\
301.4 \\
301.4 \\
301.4 \\
301.4 \\
301.4 \\
301.4 \\
301.4 \\
301.4 \\
301.4 \\
301.4 \\
301.4 \\
301.4 \\
301.5 \\
301.5 \\
301.5 \\
301.6 \\
301.7 \\
301.6 \\
301.6 \\
301.7 \\
301.6 \\
301.6 \\
301.7 \\
301.8\end{array}$ \\
\hline
\end{tabular}




\begin{tabular}{|c|c|c|c|c|c|c|c|c|c|}
\hline $\begin{array}{l}2: 49: 10 \\
2: 49: 28 \\
2: 49: 46 \\
2: 50: 5 \\
2: 50: 23 \\
2: 50: 41 \\
2: 52: 49 \\
2: 53: 7 \\
2: 53: 26 \\
2: 53: 44 \\
2: 54: 2 \\
2: 54: 29 \\
2: 54: 57 \\
2: 55: 25 \\
2: 55: 43 \\
2: 56: 1 \\
2: 56: 19 \\
2: 56: 37 \\
2: 56: 56 \\
2: 57: 14 \\
2: 57: 32 \\
2: 57: 50 \\
2: 58: 99 \\
2: 58: 27 \\
2: 58: 55 \\
2: 59: 13 \\
2: 59: 31 \\
2: 59: 49 \\
2: 59: 58 \\
3: 0: 7 \\
3: 0: 17 \\
3: 0: 26 \\
3: 0: 35 \\
3: 0: 44 \\
3: 0: 53 \\
3: 1: 2 \\
3: 1: 11 \\
3: 1: 20 \\
3: 1: 29 \\
3: 1: 39 \\
3: 1: 48 \\
3: 1: 57 \\
3: 2: 6 \\
3: 2: 15 \\
3: 2: 25 \\
3: 2: 34 \\
3: 2: 43 \\
3: 2: 52 \\
3: 3: 19 \\
3: 3: 19\end{array}$ & $\begin{array}{l}942.7 \\
940.7 \\
938.7 \\
936.7 \\
934.8 \\
932.9 \\
934.2 \\
935.5 \\
936.6 \\
938.0 \\
939.4 \\
941.0 \\
942.6 \\
944.6 \\
946.0 \\
947.5 \\
949.0 \\
950.4 \\
951.8 \\
953.5 \\
955.0 \\
956.6 \\
958.0 \\
959.3 \\
961.4 \\
962.5 \\
963.9 \\
965.3 \\
966.0 \\
966.5 \\
966.6 \\
966.8 \\
967.2 \\
967.4 \\
967.6 \\
967.8 \\
968.2 \\
968.4 \\
968.7 \\
968.9 \\
969.1 \\
969.4 \\
969.6 \\
969.7 \\
969.9 \\
969.5 \\
969.6 \\
969.4 \\
969.6 \\
969.8 \\
969.7\end{array}$ & 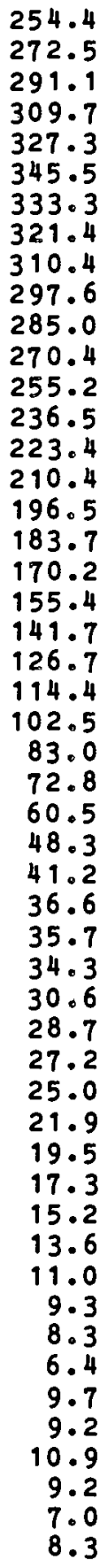 & $\begin{array}{l}23.8 \\
23.9 \\
24.0 \\
23.9 \\
23.8 \\
23.8 \\
23.6 \\
23.6 \\
23.6 \\
23.5 \\
23.6 \\
23.8 \\
23.8 \\
23.5 \\
23.4 \\
23.5 \\
23.4 \\
23.4 \\
23.5 \\
23.5 \\
23.6 \\
23.9 \\
23.9 \\
23.8 \\
24.0 \\
24.0 \\
24.0 \\
24.3 \\
24.2 \\
24.6 \\
24.8 \\
24.8 \\
24.9 \\
24.9 \\
24.9 \\
24.9 \\
24.8 \\
24.8 \\
24.9 \\
24.8 \\
24.9 \\
24.8 \\
24.3 \\
23.7 \\
22.6 \\
21.4 \\
20.9 \\
20.6 \\
20.5 \\
20.7 \\
20.6\end{array}$ & $\begin{array}{l}12.9 \\
12.9 \\
12.9 \\
12.5 \\
12.1 \\
12.0 \\
11.8 \\
11.9 \\
12.1 \\
12.3 \\
12.6 \\
12.8 \\
12.8 \\
12.8 \\
12.5 \\
12.4 \\
12.4 \\
12.4 \\
12.5 \\
12.4 \\
12.5 \\
12.6 \\
12.6 \\
12.7 \\
12.8 \\
12.8 \\
12.8 \\
12.9 \\
12.8 \\
12.9 \\
13.0 \\
13.0 \\
13.1 \\
13.1 \\
13.1 \\
13.1 \\
13.1 \\
13.1 \\
13.1 \\
13.1 \\
13.1 \\
13.3 \\
13.0 \\
12.9 \\
12.8 \\
12.5 \\
12.0 \\
11.8 \\
11.6 \\
11.6 \\
11.6\end{array}$ & $\begin{array}{l}27.2 \\
26.7 \\
26.4 \\
24.8 \\
22.9 \\
22.4 \\
22.5 \\
22.3 \\
23.5 \\
25.2 \\
26.7 \\
26.7 \\
26.7 \\
27.8 \\
26.3 \\
25.7 \\
25.8 \\
25.8 \\
25.8 \\
25.3 \\
25.5 \\
24.5 \\
24.9 \\
25.8 \\
25.5 \\
25.4 \\
25.1 \\
24.6 \\
24.5 \\
23.5 \\
23.3 \\
23.5 \\
23.6 \\
23.5 \\
23.5 \\
23.8 \\
23.8 \\
23.8 \\
23.8 \\
23.9 \\
23.8 \\
24.7 \\
25.3 \\
26.7 \\
31.0 \\
34.1 \\
33.5 \\
33.3 \\
32.8 \\
31.8 \\
31.9\end{array}$ & $\begin{array}{l}5.3 \\
5.3 \\
5.3 \\
4.9 \\
4.5 \\
4.4 \\
4.4 \\
4.4 \\
4.6 \\
4.9 \\
5.2 \\
5.3 \\
5.2 \\
5.3 \\
5.0 \\
4.9 \\
4.9 \\
4.9 \\
4.9 \\
4.8 \\
4.9 \\
4.8 \\
4.8 \\
5.0 \\
4.9 \\
5.0 \\
4.9 \\
4.9 \\
4.8 \\
4.7 \\
4.7 \\
4.8 \\
4.8 \\
4.8 \\
4.8 \\
4.9 \\
4.8 \\
4.8 \\
4.8 \\
4.9 \\
4.8 \\
5.0 \\
5.0 \\
5.1 \\
5.5 \\
5.6 \\
5.4 \\
5.2 \\
5.1 \\
5.0 \\
5.0\end{array}$ & $\begin{array}{r}58.3 \\
37.5 \\
40.8 \\
41.9 \\
39.2 \\
42.0 \\
35.0 \\
37.2 \\
38.8 \\
27.8 \\
47.0 \\
45.9 \\
34.2 \\
60.9 \\
13.3 \\
28.9 \\
32.2 \\
49.0 \\
62.0 \\
50.4 \\
34.9 \\
73.4 \\
48.8 \\
46.8 \\
125.3 \\
110.3 \\
89.7 \\
111.0 \\
152.5 \\
103.7 \\
138.5 \\
143.3 \\
180.7 \\
163.6 \\
135.3 \\
172.5 \\
163.6 \\
182.5 \\
204.2 \\
200.7 \\
200.3 \\
214.1 \\
222.8 \\
222.3 \\
229.2 \\
235.8 \\
234.7 \\
239.4 \\
241.5 \\
231.4 \\
233.9\end{array}$ & $\begin{array}{l}0.9 \\
0.9 \\
0.9 \\
0.9 \\
0.9 \\
1.0 \\
1.3 \\
1.2 \\
0.9 \\
0.6 \\
1.0 \\
1.5 \\
0.9 \\
0.2 \\
0.4 \\
0.6 \\
0.4 \\
0.3 \\
0.5 \\
0.4 \\
0.3 \\
0.8 \\
0.9 \\
-0.1 \\
0.7 \\
0.6 \\
1.1 \\
0.9 \\
0.9 \\
0.5 \\
0.7 \\
0.8 \\
0.4 \\
0.9 \\
0.7 \\
0.4 \\
0.8 \\
0.7 \\
0.7 \\
0.9 \\
1.3 \\
1.9 \\
2.3 \\
1.9 \\
1.7 \\
0.6 \\
0.9 \\
0.9 \\
0.8 \\
0.8 \\
1.2\end{array}$ & 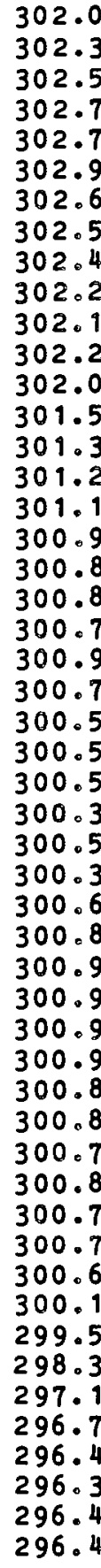 \\
\hline
\end{tabular}


July 2-3e 1981: 0311-0328 PST

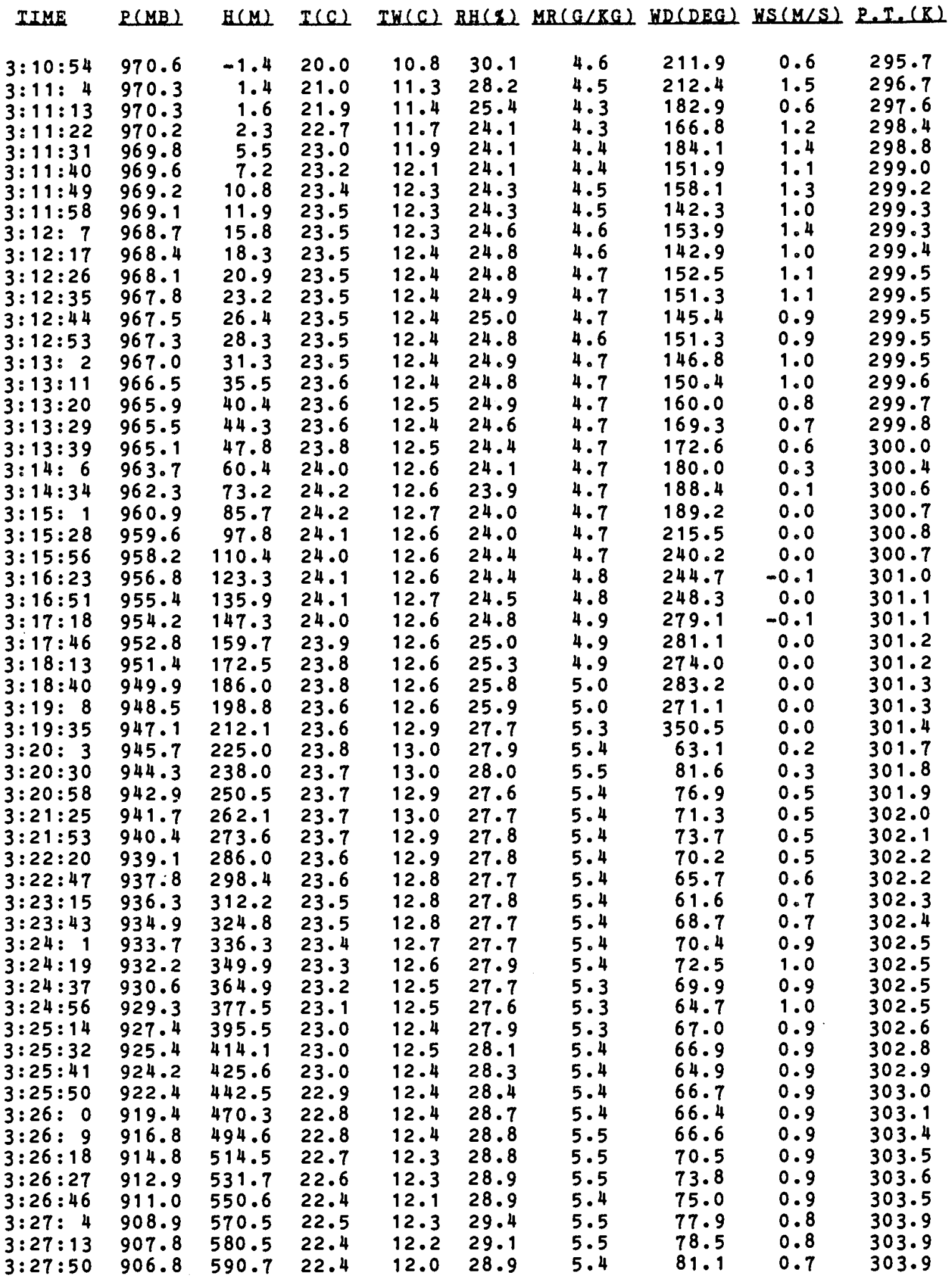


Iuly 2-3. 1981: 0353-0407 PST

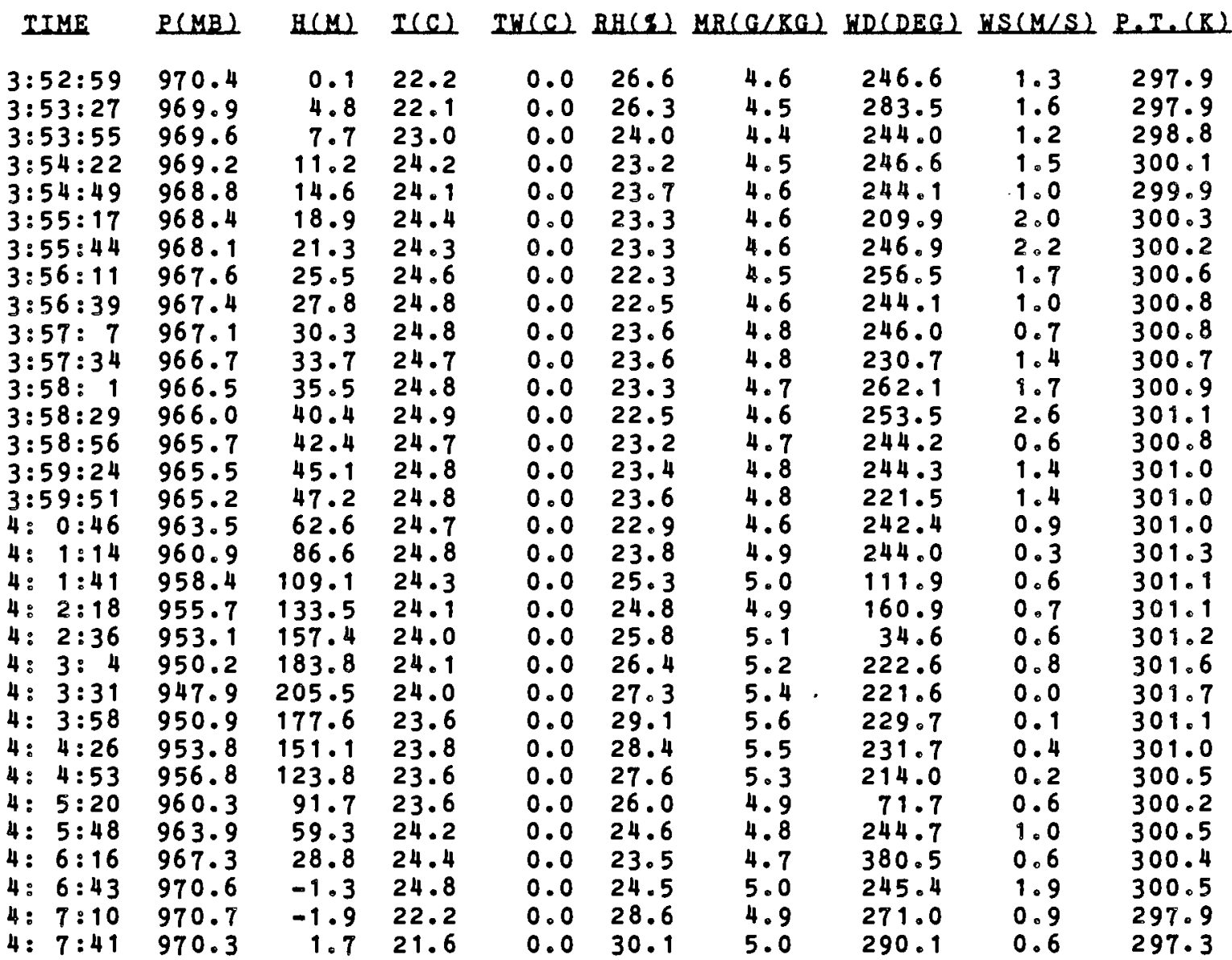


October 16. 1981: 1958-2032 RST

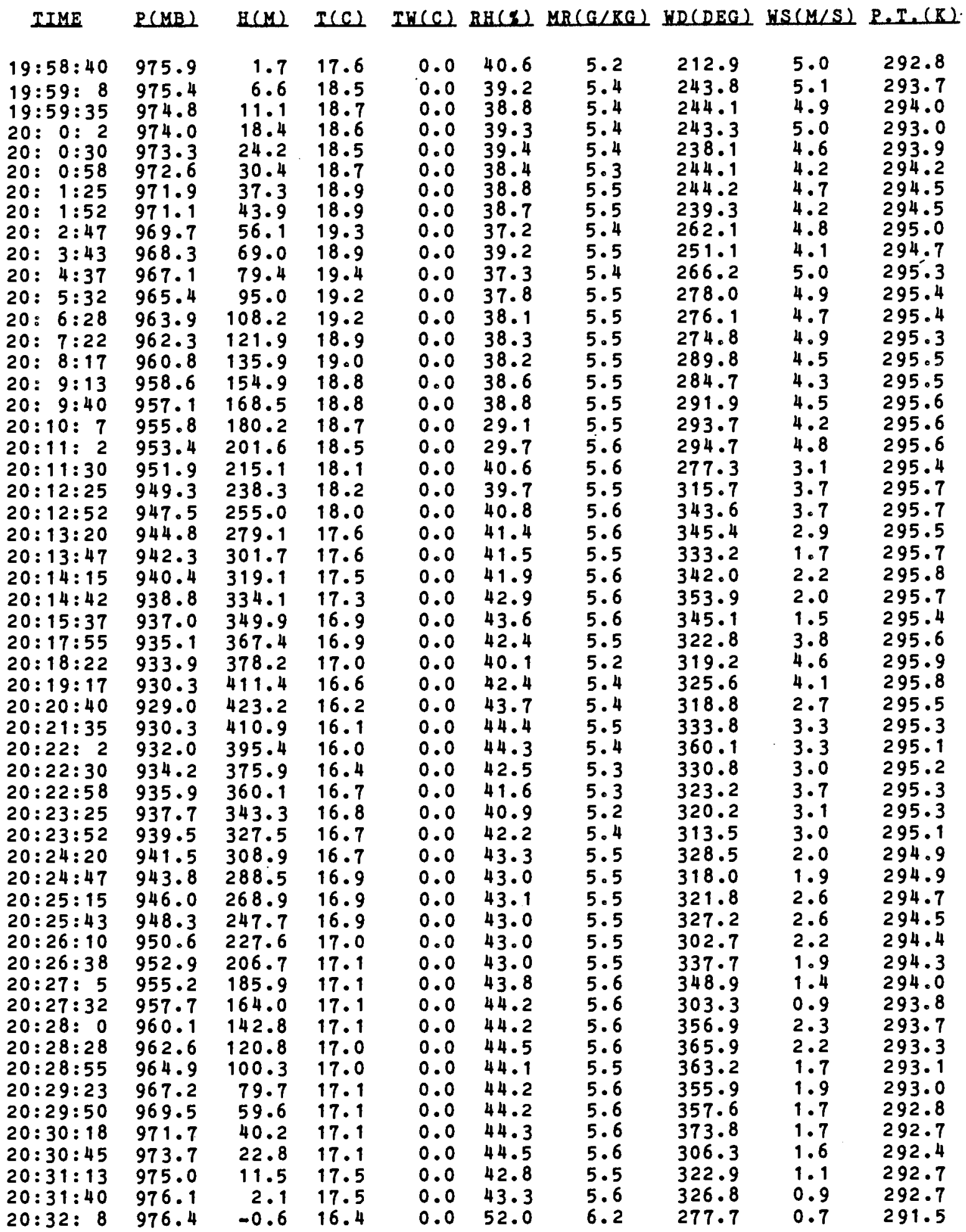


ectober 16. 1981: 2054-2111 RST

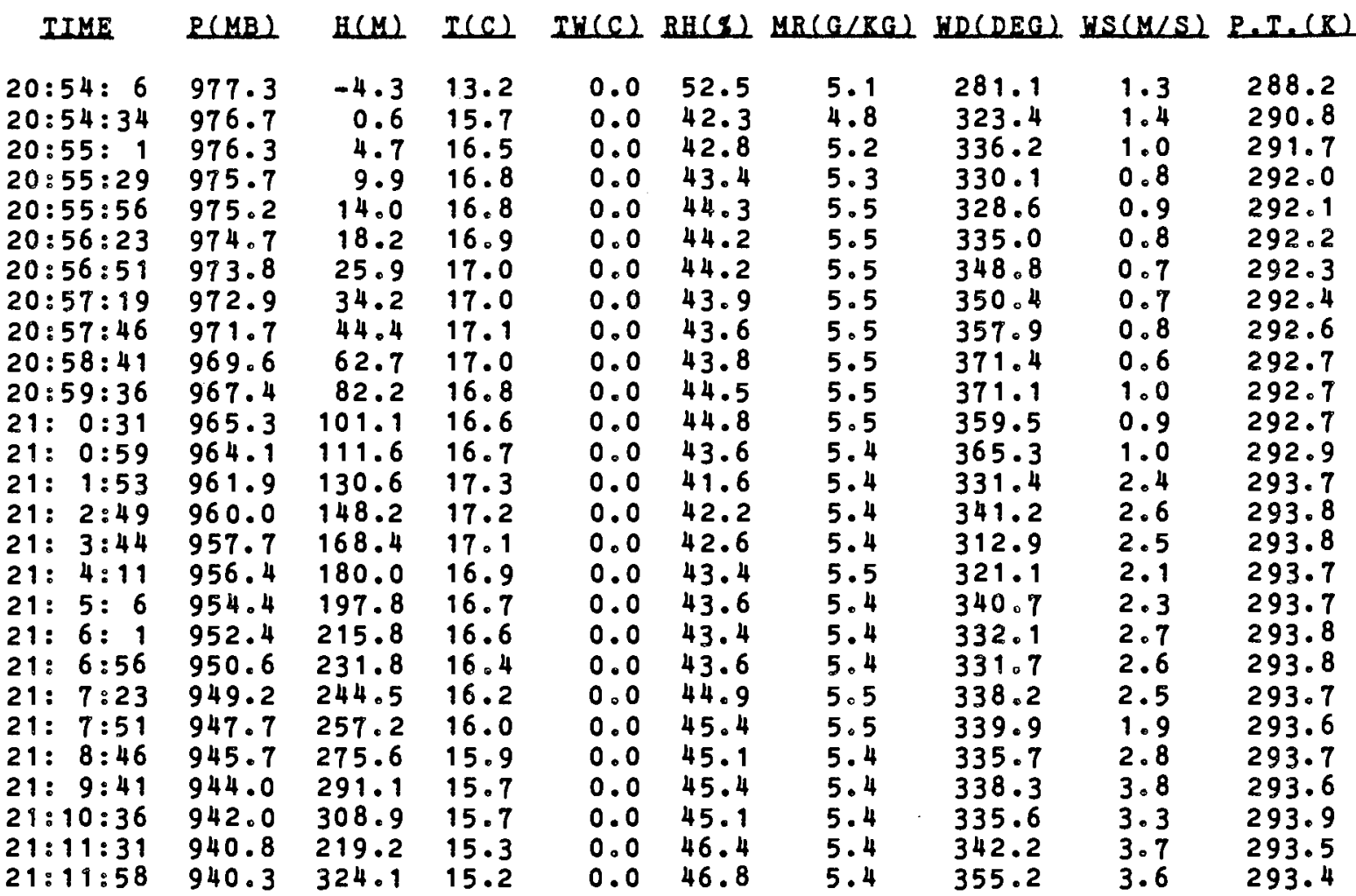


VI. Telemetry Data

The wind speed and direction data from the Hanford Telemetry Network

are listed below for the four cases. For each hour the data for all

working stations are reported as: wind direction (deg); wind speed

(mph). The data are hourly averages for the hour ending at the time indicated.

June 19-20, 1980 (Days 171-172)

$\begin{array}{rrrrrrrr}\text { Statien } & 1600 & 1700 & 1800 & 1900 & 2000 & 2100 & 2200 \\ 1 & 106 ; 5 & 112 ; 5 & 22 ; 5 & 359 ; 5 & 353 ; 5 & 329 ; 4 & 317 ; 6 \\ 2 & 47 ; 3 & 91 ; 3 & 66 ; 4 & 354 ; 4 & 272 ; 6 & 281 ; 7 & 287 ; 7 \\ 3 & 139 ; 4 & 26 ; 3 & 74 ; 2 & 67 ; 1 & 353 ; 3 & 285 ; 3 & 265 ; 5 \\ 4 & 154 ; 4 & 138 ; 4 & 69 ; 3 & 21 ; 4 & 351 ; 6 & 291 ; 5 & 270 ; 8 \\ 5 & 114 ; 4 & 134 ; 3 & 126 ; 1 & 150 ; 1 & 346 ; 2 & 336 ; 3 & 320 ; 3 \\ 6 & 111 ; 2 & 84 ; 2 & 65 ; 1 & 41 ; 1 & 23 ; 0 & 24 ; 1 & 27 ; 1 \\ 8 & 105 ; 5 & 139 ; 4 & 150 ; 3 & 119 ; 2 & 41 ; 4 & 39 ; 5 & 326 ; 5 \\ 9 & 81 ; 4 & 86 ; 5 & 80 ; 3 & 354 ; 2 & 332 ; 3 & 2 ; 4 & 349 ; 2 \\ 11 & 102 ; 6 & 59 ; 3 & 75 ; 2 & 31 ; 3 & 10 ; 3 & 240 ; 1 & 304 ; 4 \\ 14 & 157 ; 9 & 165 ; 9 & 175 ; 7 & 193 ; 6 & 201 ; 4 & 188 ; 4 & 173 ; 4 \\ 15 & 142 ; 3 & 146 ; 2 & 18 ; 2 & 317 ; 3 & 352 ; 4 & 11 ; 4 & 32 ; 8 \\ 20 & 13 ; 6 & 28 ; 4 & 358 ; 4 & 352 ; 6 & 5 ; 7 & 19 ; 12 & 0 ; 9 \\ & & & & & & \\ \text { Stat10n } & 2300 & 0000 & 0100 & 0200 & 0300 & 0400 & 0500 \\ 1 & 345 ; 6 & 326 ; 7 & 333 ; 6 & 341 ; 5 & 339 ; 7 & 287 ; 4 & 292 ; 3 \\ 2 & 283 ; 7 & 283 ; 7 & 281 ; 5 & 278 ; 5 & 278 ; 6 & 294 ; 7 & 298 ; 7 \\ 3 & 303 ; 3 & 289 ; 5 & 283 ; 5 & 291 ; 5 & 303 ; 5 & 308 ; 5 & 281 ; 4 \\ 4 & 264 ; 9 & 263 ; 8 & 269 ; 8 & 263 ; 9 & 265 ; 8 & 275 ; 8 & 273 ; 10 \\ 5 & 226 ; 1 & 288 ; 4 & 305 ; 5 & 245 ; 0 & 290 ; 1 & 237 ; 0 & 265 ; 1 \\ 6 & 268 ; 2 & 304 ; 2 & 305 ; 4 & 264 ; 5 & 303 ; 5 & 293 ; 7 & 289 ; 7 \\ 8 & 303 ; 5 & 307 ; 1 & 102 ; 6 & 341 ; 4 & 305 ; 5 & 305 ; 3 & 264 ; 5 \\ 9 & 318 ; 4 & 307 ; 3 & 268 ; 2 & 298 ; 5 & 328 ; 5 & 338 ; 4 & 328 ; 2 \\ 11 & 261 ; 4 & 323 ; 4 & 330 ; 2 & 341 ; 7 & 340 ; 8 & 304 ; 3 & 294 ; 5 \\ 14 & 203 ; 5 & 197 ; 5 & 195 ; 4 & 197 ; 4 & 203 ; 5 & 197 ; 5 & 192 ; 5 \\ 15 & 43 ; 9 & 59 ; 10 & 48 ; 6 & 39 ; 6 & 14 ; 5 & 351 ; 5 & 5 ; 6 \\ 20 & 8 ; 6 & 32 ; 11 & 28 ; 9 & 9 ; 4 & 1 ; 5 & 6 ; 7 & 11 ; 9\end{array}$


JuIy 1-2, 1980 (Days 183-184)

\begin{tabular}{|c|c|c|c|c|c|c|c|}
\hline Station & 1600 & 1700 & 1800 & 1900 & 2000 & 2100 & 2200 \\
\hline $\begin{array}{r}2 \\
3 \\
4 \\
5 \\
6 \\
8 \\
9 \\
14 \\
15 \\
20\end{array}$ & $\begin{array}{r}--- \\
135 ; 6 \\
98 ; 5 \\
39 ; 5 \\
22 ; 2 \\
143 ; 5 \\
55 ; 6 \\
91 ; 4 \\
10 ; 5 \\
289 ; 5\end{array}$ & $\begin{array}{r}87 ; 3 \\
143 ; 6 \\
75 ; 8 \\
352 ; 5 \\
67 ; 4 \\
134 ; 5 \\
65 ; 8 \\
95 ; 5 \\
62 ; 5 \\
318 ; 3\end{array}$ & $\begin{array}{r}144 ; 8 \\
153 ; 6 \\
82 ; 9 \\
87 ; 6 \\
78 ; 6 \\
99 ; 4 \\
89 ; 8 \\
100.6 \\
93 ; 5 \\
119 ; 5\end{array}$ & $\begin{array}{c}148 ; 6 \\
140 ; 11 \\
96 ; 9 \\
87 ; 5 \\
95 ; 7 \\
104 ; 4 \\
120 ; 7 \\
107 ; 6 \\
124 ; 4 \\
143 ; 7\end{array}$ & $\begin{aligned} 201 ; 5 \\
162 ; 10 \\
80 ; 4 \\
124 ; 5 \\
96 ; 5 \\
103 ; 5 \\
127 ; 3 \\
147 ; 5 \\
101 ; 4 \\
165 ; 7\end{aligned}$ & $\begin{array}{r}206 ; 6 \\
177 ; 7 \\
310 ; 4 \\
190 ; 1 \\
124 ; 4 \\
79 ; 5 \\
73 ; 2 \\
161 ; 5 \\
72 ; 5 \\
198 ; 9\end{array}$ & $\begin{array}{r}211 ; 8 \\
182 ; 4 \\
272 ; 7 \\
210 ; 1 \\
136 ; 1 \\
48 ; 3 \\
210 ; 2 \\
183 ; 5 \\
76 ; 6 \\
219 ; 13\end{array}$ \\
\hline Station & 2300 & 0000 & 0100 & 0200 & 0300 & 0400 & 0500 \\
\hline $\begin{array}{r}2 \\
3 \\
4 \\
5 \\
6 \\
8 \\
9 \\
14 \\
15 \\
20\end{array}$ & $\begin{array}{r}222 ; 8 \\
210 ; 6 \\
277 ; 7 \\
253 ; 1 \\
268 ; 2 \\
40 ; 3 \\
259 ; 3 \\
167 ; 5 \\
73 ; 5 \\
235 ; 18\end{array}$ & $\begin{array}{r}234 ; 7 \\
221 ; 5 \\
301 ; 6 \\
279 ; 1 \\
296 ; 2 \\
0 ; 2 \\
325 ; 6 \\
176 ; 5 \\
48 ; 3 \\
238 ; 19\end{array}$ & $\begin{array}{r}216 ; 3 \\
205 ; 5 \\
276 ; 7 \\
173 ; 1 \\
263 ; 2 \\
69 ; 3 \\
115 ; 4 \\
178 ; 5 \\
149 ; 4 \\
242 ; 18\end{array}$ & $\begin{array}{l}233 ; 4 \\
169 ; 5 \\
271 ; 7 \\
211 ; 1 \\
117 ; 5 \\
306 ; 4 \\
316 ; 3 \\
186 ; 5 \\
112 ; 2 \\
239 ; 21\end{array}$ & $\begin{array}{r}261 ; 6 \\
225 ; 4 \\
312 ; 6 \\
304 ; 2 \\
270 ; 3 \\
51 ; 3 \\
72 ; 3 \\
152 ; 6 \\
104 ; 4 \\
242 ; 16\end{array}$ & $\begin{array}{r}304 ; 5 \\
209 ; 6 \\
303 ; 6 \\
234 ; 0 \\
291 ; 2 \\
321 ; 3 \\
323 ; 4 \\
186 ; 6 \\
98 ; 5 \\
274 ; 9\end{array}$ & $\begin{array}{r}296 ; 5 \\
205 ; 4 \\
287 ; 7 \\
145 ; 2 \\
279 ; 4 \\
300 ; 4 \\
92 ; 3 \\
179 ; 6 \\
218 ; 2 \\
282 ; 11\end{array}$ \\
\hline
\end{tabular}


July 2-3, 1981 (Days 183-184)

\begin{tabular}{|c|c|c|c|c|c|c|c|}
\hline Station & 1600 & 1700 & 1800 & 1900 & 2000 & 2100 & 2200 \\
\hline $\begin{array}{l}1 \\
2 \\
3 \\
4 \\
5 \\
6 \\
7 \\
8 \\
9 \\
10 \\
11 \\
12 \\
13 \\
14 \\
15 \\
16 \\
18 \\
20\end{array}$ & $\begin{array}{r}82 ; 3 \\
24 ; 5 \\
356 ; 4 \\
344 ; 6 \\
83 ; 3 \\
11 ; 4 \\
328 ; 5 \\
104 ; 6 \\
257 ; 3 \\
278 ; 2 \\
24 ; 4 \\
318 ; 4 \\
311 ; 4 \\
52 ; 4 \\
321 ; 7 \\
28 ; 6 \\
13 ; 5 \\
46 ; 11\end{array}$ & $\begin{array}{r}126 ; 4 \\
16 ; 5 \\
14 ; 4 \\
8 ; 6 \\
304 ; 3 \\
27 ; 3 \\
333 ; 5 \\
119 ; 5 \\
256 ; 3 \\
292 ; 2 \\
29 ; 4 \\
314 ; 3 \\
298 ; 3 \\
18 ; 4 \\
327 ; 6 \\
13 ; 6 \\
102 ; 4 \\
48 ; 9\end{array}$ & $\begin{array}{r}179 ; 3 \\
12 ; 5 \\
332 ; 4 \\
341 ; 6 \\
313 ; 4 \\
356 ; 4 \\
354 ; 5 \\
93 ; 5 \\
253 ; 2 \\
290 ; 3 \\
27 ; 6 \\
278 ; 3 \\
341 ; 5 \\
14 ; 3 \\
341 ; 6 \\
340 ; 8 \\
127 ; 5 \\
39 ; 9\end{array}$ & $\begin{array}{r}227 ; 1 \\
139 ; 5 \\
337 ; 4 \\
344 ; 7 \\
315 ; 7 \\
340 ; 6 \\
325 ; 6 \\
98 ; 3 \\
249 ; 2 \\
298 ; 3 \\
24 ; 5 \\
299 ; 5 \\
283 ; 4 \\
5 ; 3 \\
356 ; 4 \\
329 ; 9 \\
178 ; 3 \\
72 ; 6\end{array}$ & $\begin{array}{r}169 ; 2 \\
276 ; 9 \\
316 ; 5 \\
319 ; 7 \\
323 ; 5 \\
311 ; 5 \\
302 ; 6 \\
60 ; 3 \\
248 ; 2 \\
286 ; 3 \\
201 ; 3 \\
306 ; 8 \\
293 ; 4 \\
328 ; 4 \\
21 ; 3 \\
310 ; 8 \\
193 ; 3 \\
20 ; 4\end{array}$ & $\begin{array}{l}317 ; 6 \\
278 ; 9 \\
293 ; 5 \\
254 ; 9 \\
310 ; 7 \\
292 ; 6 \\
294 ; 5 \\
352 ; 5 \\
132 ; 2 \\
272 ; 5 \\
323 ; 3 \\
275 ; 7 \\
296 ; 3 \\
320 ; 4 \\
355 ; 4 \\
320 ; 10 \\
291 ; 4 \\
211 ; 2\end{array}$ & \begin{tabular}{l}
$350 ; 5$ \\
$277 ; 7$ \\
$305 ; 6$ \\
$249 ; 10$ \\
$288 ; 4$ \\
$276 ; 4$ \\
$302 ; 6$ \\
$334 ; 4$ \\
$226 ; 1$ \\
$262 ; 6$ \\
$345 ; 6$ \\
$289 ; 6$ \\
\hdashline$-2--$ \\
$329 ; 3$ \\
$331 ; 4$ \\
$356 ; 7$ \\
$266 ; 4$ \\
$214 ; 2$
\end{tabular} \\
\hline Station & 2300 & 0000 & 0100 & 0200 & 0300 & 0400 & 0500 \\
\hline $\begin{array}{l}1 \\
2 \\
3 \\
4 \\
5 \\
6 \\
7 \\
8 \\
9 \\
10 \\
11 \\
12 \\
13 \\
14 \\
15 \\
16 \\
18 \\
20\end{array}$ & $\begin{array}{l}333 ; 5 \\
279 ; 8 \\
317 ; 6 \\
250 ; 11 \\
136 ; 3 \\
269 ; 5 \\
303 ; 4 \\
328 ; 3 \\
263 ; 5 \\
264 ; 6 \\
213 ; 3 \\
299 ; 5 \\
237 ; 3 \\
54 ; 4 \\
28 ; 7 \\
345 ; 4 \\
253 ; 3 \\
213 ; 2\end{array}$ & $\begin{array}{l}331 ; 3 \\
282 ; 7 \\
301 ; 4 \\
245 ; 11 \\
144 ; 3 \\
270 ; 5 \\
313 ; 7 \\
281 ; 3 \\
263 ; 6 \\
263 ; 6 \\
235 ; 3 \\
158 ; 1 \\
212 ; 2 \\
42 ; 4 \\
31 ; 6 \\
142 ; 2 \\
250 ; 3 \\
212 ; 2\end{array}$ & $\begin{array}{r}288 ; 2 \\
270 ; 3 \\
151 ; 1 \\
241 ; 12 \\
180 ; 3 \\
163 ; 4 \\
254 ; 3 \\
69 ; 4 \\
257 ; 3 \\
255 ; 4 \\
256 ; 2 \\
248 ; 3 \\
181 ; 2 \\
25 ; 3 \\
54 ; 4 \\
122 ; 6 \\
293 ; 4 \\
214 ; 2\end{array}$ & $\begin{array}{r}275 ; 1 \\
266 ; 3 \\
259 ; 3 \\
249 ; 8 \\
191 ; 4 \\
210 ; 4 \\
224 ; 5 \\
73 ; 5 \\
252 ; 2 \\
264 ; 5 \\
245 ; 2 \\
199 ; 4 \\
256 ; 1 \\
75 ; 3 \\
162 ; 5 \\
187 ; 4 \\
313 ; 2 \\
215 ; 2\end{array}$ & $\begin{array}{l}303 ; 2 \\
247 ; 3 \\
343 ; 3 \\
246 ; 12 \\
152 ; 2 \\
217 ; 1 \\
290 ; 3 \\
86 ; 4 \\
253 ; 2 \\
274 ; 7 \\
221 ; 2 \\
115 ; 2 \\
320 ; 1 \\
155 ; 1 \\
129 ; 5 \\
205 ; 6 \\
206 ; 3 \\
217 ; 1\end{array}$ & $\begin{array}{r}350 ; 3 \\
286 ; 2 \\
317 ; 1 \\
246 ; 7 \\
290 ; 3 \\
205 ; 1 \\
354 ; 4 \\
300 ; 3 \\
186 ; 1 \\
34 ; 2 \\
158 ; 5 \\
246 ; 3 \\
187 ; 2 \\
157 ; 1 \\
124 ; 7 \\
157 ; 3 \\
152 ; 3 \\
225 ; 1\end{array}$ & $\begin{array}{r}347 ; 6 \\
317 ; 4 \\
295 ; 3 \\
222 ; 3 \\
187 ; 2 \\
140 ; 3 \\
254 ; 2 \\
64 ; 4 \\
134 ; 1 \\
257 ; 3 \\
130 ; 4 \\
258 ; 3 \\
236 ; 1 \\
338 ; 1 \\
133 ; 6 \\
200 ; 4 \\
146 ; 2 \\
224 ; 4\end{array}$ \\
\hline
\end{tabular}


October 16-17, 1981 (Days 289-290)

\begin{tabular}{|c|c|c|c|c|c|c|c|}
\hline Station & 1400 & 1500 & 1600 & 1700 & 1800 & 1900 & 2000 \\
\hline $\begin{array}{l}1 \\
2 \\
3 \\
4 \\
5 \\
6 \\
7 \\
8 \\
9 \\
11 \\
13 \\
14 \\
15 \\
16 \\
18 \\
20\end{array}$ & $\begin{array}{c}169 ; 6 \\
218 ; 12 \\
93 ; 6 \\
91 ; 3 \\
84 ; 6 \\
117 ; 4 \\
110 ; 2 \\
108 ; 4 \\
92 ; 2 \\
155 ; 5 \\
114 ; 3 \\
63 ; 2 \\
180 ; 5 \\
170 ; 6 \\
126 ; 5 \\
233 ; 13\end{array}$ & $\begin{array}{r}159 ; 6 \\
296 ; 5 \\
87 ; 9 \\
54 ; 3 \\
88 ; 7 \\
107 ; 4 \\
76 ; 3 \\
98 ; 3 \\
92 ; 2 \\
161 ; 5 \\
0 ; 6 \\
60 ; 2 \\
177 ; 4 \\
101 ; 4 \\
132 ; 5 \\
231 ; 10\end{array}$ & $\begin{array}{r}163 ; 6 \\
253 ; 5 \\
105 ; 10 \\
60 ; 2 \\
104 ; 7 \\
120 ; 6 \\
83 ; 3 \\
89 ; 3 \\
94 ; 2 \\
137 ; 7 \\
5 ; 5 \\
158 ; 6 \\
142 ; 6 \\
116 ; 6 \\
134 ; 5 \\
227 ; 7\end{array}$ & $\begin{array}{r}173 ; 5 \\
249 ; 4 \\
131 ; 6 \\
251 ; 4 \\
110 ; 5 \\
123 ; 5 \\
34 ; 4 \\
67 ; 3 \\
0-2 \\
144 ; 5 \\
1 ; 4 \\
152 ; 6 \\
144 ; 6 \\
137 ; 5 \\
184 ; 4 \\
235 ; 6\end{array}$ & $\begin{array}{r}188 ; 4 \\
278 ; 7 \\
294 ; 3 \\
299 ; 6 \\
125 ; 3 \\
334 ; 4 \\
328 ; 6 \\
328 ; 3 \\
92 ; 1 \\
142 ; 5 \\
332 ; 4 \\
165 ; 4 \\
148 ; 5 \\
159 ; 8 \\
180 ; 3 \\
285 ; 7\end{array}$ & $\begin{array}{l}308 ; 4 \\
296 ; 9 \\
311 ; 6 \\
236 ; 5 \\
311 ; 5 \\
304 ; 7 \\
296 ; 6 \\
321 ; 3 \\
284 ; 1 \\
143 ; 3 \\
309 ; 3 \\
214 ; 6 \\
203 ; 4 \\
342 ; 10 \\
279 ; 2 \\
313 ; 10\end{array}$ & $\begin{array}{l}309 ; 4 \\
295 ; 7 \\
318 ; 3 \\
248 ; 10 \\
313 ; 1 \\
272 ; 6 \\
329 ; 3 \\
10 ; 2 \\
306 ; 0 \\
221 ; 1 \\
268 ; 2 \\
223 ; 6 \\
186 ; 4 \\
336 ; 4 \\
230 ; 3 \\
276 ; 10\end{array}$ \\
\hline Statien & 2100 & 2200 & 2300 & 2000 & 0100 & 2200 & 0300 \\
\hline $\begin{array}{l}1 \\
2 \\
3 \\
4 \\
5 \\
6 \\
7 \\
8 \\
9 \\
11 \\
13 \\
14 \\
15 \\
16 \\
18 \\
20\end{array}$ & $\begin{array}{r}1 ; 2 \\
306 ; 3 \\
297 ; 4 \\
250 ; 6 \\
304 ; 3 \\
305 ; 4 \\
324 ; 3 \\
292 ; 7 \\
325 ; 1 \\
131 ; 2 \\
56 ; 1 \\
176 ; 3 \\
144 ; 4 \\
359 ; 5 \\
120 ; 2 \\
328 ; 13\end{array}$ & $\begin{array}{r}285 ; 2 \\
308 ; 7 \\
325 ; 3 \\
247 ; 5 \\
254 ; 1 \\
272 ; 2 \\
215 ; 3 \\
269 ; 5 \\
51 ; 1 \\
258 ; 2 \\
223 ; 2 \\
159 ; 4 \\
228 ; 3 \\
332 ; 3 \\
218 ; 2 \\
341 ; 16\end{array}$ & $\begin{array}{c}33 ; 2 \\
314 ; 11 \\
326 ; 4 \\
246 ; 7 \\
303 ; 2 \\
299 ; 4 \\
350 ; 3 \\
219 ; 2 \\
54 ; 1 \\
242 ; 2 \\
304 ; 2 \\
160 ; 3 \\
217 ; 2 \\
336 ; 5 \\
11 ; 3 \\
354 ; 18\end{array}$ & $\begin{array}{c}344 ; 4 \\
319 ; 11 \\
27 ; 3 \\
254 ; 4 \\
291 ; 3 \\
298 ; 5 \\
348 ; 5 \\
135 ; 3 \\
80 ; 3 \\
332 ; 4 \\
249 ; 1 \\
-\infty--- \\
-\infty--- \\
----- \\
---\infty \\
358 ; 17\end{array}$ & $\begin{array}{r}341 ; 7 \\
308 ; 10 \\
24 ; 3 \\
249 ; 5 \\
155 ; 1 \\
221 ; 4 \\
198 ; 2 \\
286 ; 2 \\
322 ; 1 \\
298 ; 2 \\
272 ; 2 \\
313 ; 1 \\
330 ; 2 \\
41 ; 6 \\
301 ; 3 \\
2 ; 15\end{array}$ & $\begin{array}{r}353 ; 6 \\
312 ; 10 \\
304 ; 2 \\
244 ; 4 \\
291 ; 2 \\
284 ; 3 \\
341 ; 2 \\
298 ; 4 \\
50 ; 1 \\
311 ; 2 \\
315 ; 1 \\
320 ; 2 \\
32 ; 6 \\
27 ; 5 \\
335 ; 3 \\
8 ; 21\end{array}$ & $\begin{array}{r}3 ; 3 \\
330 ; 9 \\
326 ; 4 \\
191 ; 3 \\
14 ; 1 \\
280 ; 4 \\
4 ; 1 \\
330 ; 4 \\
77 ; 1 \\
338 ; 3 \\
263 ; 1 \\
336 ; 2 \\
40 ; 9 \\
261 ; 2 \\
253 ; 2 \\
11 ; 20\end{array}$ \\
\hline
\end{tabular}


PNL -4406

UC- 11

ASCOT-82-5

\section{DISTRIBUTION}

No. of

Copies

\section{OFFSITE}

DOE Office of Health and Environmental Research washington, DC 20545

DOE Technical Information Center

R. F. Abbey, Jr.

Hydrology \& Meteorology Branch

U.S. Nuclear Regulatory Commission

Bethesda, MD 20555

D. S. Ballantine

U.S. Department of Energy Mail stop E-74

Washington, D.C. 20545

Sumner Barr

Environmental studies Group

MS-D466

Los Alamos National Lab

P.O. Box 1663

Los Alamos, NM 87544

M. L. Barad

50 Munroe Street

Belmont, MA 02178

James Bergen

RWU 1601

Pacific S.W. Forest and

Range Experiment station

P.O. Box 245

Berkeley, CA 94701

Dr. G. E. Bertolin

Supervisor, Air Studies

Rio Blanco oil Shale Company

2851 So. Parker Rd.

Aurora, CO 80014
No. of

Copies

Prof. A. K. Blackadar

Pennsylvania State University

Department of Meteorology

503 Walker Building

University Park, PA 16802

N. E. Bowne

TRC

125 Silas Deane Hwy.

Wethersfield, CT 06190

\section{Gary Briggs}

Meteorology MD-80

U.S. Environmental

Protection Agency

Research Triangle Park, NC 27711

Sue Bunker

Earth \& Space Sciences Div.

MS $-F 665$

Los Alamos Scientific Lab

P.O. Box 1663

Los Alamos, NM 87545

Hugh Church

Division 4533

Sandia Laboratory

P.O. Box 5800

Albuquerque, NM 87115

Mr. Cionco, Commander/Director

Atmospheric Laboratory

U.S. Army Electronics Command

DRIAS-VE-C

White Sands Missile Range, NM 88002 
No. of

Copies

Bill Clements

Earth \& Space Sciences Div.

Los Alamos Scientific Lab.

Mail stop D-466

P. O. Box 1663

Los Alamos, NM 87545

Dr. W. R. Cotton

Colorado State University

Department of Atmospheric Sciences

Ft. Collins, Co 80523

Richard Coulter

Atmospheric Physics section Argonne National Laboratory

9700 South Cass Avenue

Argonne, IL 60439

Cecil G. Davis

MS-D420 X-5

Los Alamos Scientific Lab.

P. O. Box 1663

Los Alamos, NM 87545

15 M. H. Dickerson

$L-262$

Lawrence Livermore

National Laboratory

P. O. Box 808

Livermore, CA 94550

C. R. Dickson

ARL Field Research office

NOAA

550 second street

Idaho Falls, ID 83401

Roland Draxler

National Oceanic and

Atmospheric Administration

Air Resources Laboratories

8 th Floor

6010 Executive Boulevard

Rockville, MD 20852

Dr. Ekke Dreiseitl

Institut fur Meteorologie

Universitat Innsbruck

Schopfstrasse 41

A-6020 Innsbruck

AUSTRIA
No. of

Copies

Peter Finkelstein

EMSL MD-56

U.S. EPA

Research Triangle Park, NC 27711

Rich Fisher

U.S. EPA, Region 8

1860 Lincoln Street

Denver, Co 80295

Dr. Michael Flasar

Goddard Space Flight center

Code 693

Greenbelt, MD 20771

Mike Fosberg

U.S. Forest Service

Forest Fire Laboratory

4955 Canyon Crest Drive

Riverside, CA 92507

D. G. Fox

Rocky Mountain Forest and

Range Experiment station

240 West Prospect street

Ft. Collins, CO 80526

Dr. Walter Flood

U.S. Army Research Office

ATTN : DRXRO-DP

P.O. Box 12211

Research Triangle Park, NC 27709

Al Garrett

773A-1015

Savannah River Laboratory

E. I. du Pont de Nemours, Inc.

Aiken, SC 29808

Frank Gifford

Atmospheric Turbulence and

Diffusion Laboratory

NOAA

P. O. BOX E

Oak Ridge, TN 37830

Sven-Erik Gryning

Riso National Laboratory

OK-4000 Roskilde

DENMARK

Paul Gudiksen

$\mathrm{L}-262$

Lawrence Livermore

National Laboratory

P. O. Box 808

Livermore, CA 94550 
No. of

Copies

Dr. Lev Gutman

Dept. of Geophysics and

Planetary Science

Tel Aviv University

Tel Aviv, Israel

Steve Hanna

Environmental Research and Technology, Inc.

3 Militia Drive

Lexington, MA 02173

Dr. Duane Haugen

U.S. Dept. of Commerce

NOAA

Environmental Research Lab

Boulder, CO 80302

Bruce Hicks

NOAA/Atmospheric Turbulence and Diffusion Laboratory

P.O. BoX E

Oak Ridge, TN 37830

G. R. Hilst

Electric Power

Research Institute

P.O. Box 10412

Palo Alto, CA 94303

Ray Hosker

NOAA/Atmospheric Turbulence and Diffusion Laboratory

P.O. BOX E

Oak Ridge, TN 37830

Frank Hudson

Dept. of Energy

ER-74

Washington, D.C. 20545

Dr. Andre Junod

Institut Suisse de Meteorologie

Service de la Protection de I'Air

Les Invuardes

CH-1530 Payerne

SWITZERIAND

Bill Knuth

Environmental Systems and Service

4895 Gaddy Lane

Kelseyvilie, CA 95451
No. of

Copies

Dr. H. Kolb

Institut fur Meteorologie und Geophysik

Universitat wien

Hohe Warte 38

A-1190 Wien

AUSTRIA

Mr. Larry Kronenberger

EXXON Corporation U.S.A.

P.O. Box 2180

Houston, TX 77001

Rolf Lange

$\mathrm{I}-262$

Lawrence Livermore Lab

P.O. Box 808

Livermore, CA 94550

Bob Lee

I-262

Lawrence Iivermore Lab

P.O. BOX 808

Iivermore, CA 94550

Dr. John Leone

$\mathrm{I}-262$

Lawrence Livermore Lab

P.O. Box 808

Livermore, CA 94550

Dr. P. Lehmann

Societe d'Etude de l'Environment Rue du Midi 33

$\mathrm{CH}-1800$ Vevey

SWITZERIAND

DI. Tom Lyons

Murdoch University

School of Environmental and Life Science

Murdoch

WEST AUSTRALIA 6153

P. C. Manins

CSIRO

Div. of Atmospheric Physics

P.O. Box 77

Mordialloc, victoria

AUSTRALIA 3195

Dr. Larry Mahrt

Dept. of Atmospheric Sciences

Oregon State University

Corvalis, OR 97331 
No. of

Copies

Earl Markee

Hydrology \& Meteorology Branch

U.S. Nuclear Regulatory Commission

Bethesda, MD 20555

Morris McCutchen

Rocky Mountain Forest and Range Experiment station

240 West Prospect Street

Ft. Collins, CO 80526

Tom McKee

Colorado State University

Dept. of Civil Engineering

Ft. Collins, co 80523

R. J. McNider

Alabama Air Pollution Commission

645 So. McDonough St.

Montgomery, AL 36130

C. J. Nappo

NOAA/Atmospheric Trubulence and Diffusion Laboratory

P. O. Box $E$

Oak Ridge, TN 37830

W. D. Neff

Wave Propagation Lab

NOAA/Environmental

Research Lab

M. S. R45 X7

325 Broadway

Boulder, CO 80303

Dr. Frans Nieuwstadt

Royal Netherlands

Meteorological Institute

P. O. Box 201

de Bilt,

THE NETHERLANDS

Dr. Z. Petkovsek

Fakulteta za navavoslovje

In technologije

Katedra za Meteorologijo

Ljubljana P.P. 331

Askerceva 12

YUGOSIAVIA
No. of

Copies

R. A. Pielke

Colorado state University

Dept. of Atmospheric Sciences

Ft. Collins, CO 80523

K. Shankar Rao

NOAA/Atmospheric Turbulence and Diffusion Lab

P. O. Box E

Oak Ridge, TN 37830

Dr. Hans Richner

Laboratorium fur Atmospharenphysik ETH

Honggerberg HPP

$\mathrm{CH}-8049$ Zurich

SWITZERIAND

B. L. Sawford

CSIRO

Div. of Atmospheric Physics

P. O. Box 77

Mordialloc, Victoria

AUSTRALIA 3195

Frank Schiermeier

Meteorology MD-80

U.S. Environmental Protection Agency

Research Triangle Park, NC 27711

D. S. Slade

U. S. Dept. of Energy

Office of Health and

Environmental Research MS-ER 74

Washington, D. C. 20545

Ken Spengler

American Meteorology Society

45 Beacon street

Boston, MA 02108

Gene start

NOAA

550 second street

Idaho Falls, ID 83401

Dr. Wen Tang

Dept. of Meteorology

University of Lowell

Lowe11, MA 01854 
No. of

\section{Copies}

Dr. P. D. Tyson

Dept. of Geography \& Environmental studies University of Witwatersrand Jan Smuts Avenue Johannesburg SOUTH AFRICA

Dr. Charlotte Urfer Centrale Meteorologica Svizzera

Osservatorio Ticinesse CH-6605 Locarno-Monit SWITZERLAND

M. A. Wolf

Associate Professor

Dept. of Atmospheric Sciences Oregon State University

Corvallis, OR 97331

Dr. R. M. Woods, Jr. U.S. Dept. of Energy Office of Energy Research MS ER-222

Washington, D. C. 20545

Gene L. Wooldridge

Dept. of Soil Sciences and Biometeorology

Utah State University/UMC-48

Logan, UT 84322

Dr. John Wyngaard

NCAR

P. O. Box 3000

Boulder, CO 80307

Tetsuji Yamada

Los Alamos National Lab

MS-D466

P. O. Box 1663

Los Alamos, NM 87545

\section{Barry Zalcman}

Office of Inspection

and Enforcement

U. S. Nuclear Regulatory Commission

Bethesda, MD 20555
No. of

Copies

ONSITE

DOE Richland Operations Office

H. E. Ransom

55 Pacific Northwest Laboratory

A. B. Abbey

D. B. Cearlock

G. W. Dawson

$\mathrm{J}$. C. Doran

D. W. Dragnich

C. E. Elderkin

R. R. Hadlock

J. M. Hales

T. W. Horst (3)

N. S. Laulainen

M. M. Orgill

W. T. Pennell

J. V. Ramsdell

G. A. Sehmel

WGN Slinn

J. M. Thorp

L. I. Wendell

C. D. Whiteman

$R$. $R$. Woodruff

Publishing Coordination (2)

Technical Information (5) 


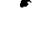

